\title{
Gewässerversauerung und \\ Fließgewässerbiozönosen im Harz
}

\author{
Dissertation \\ zur Erlangung des Doktorgrades \\ der Mathematisch-Naturwissenschaftlichen Fachbereiche \\ der Georg-August-Universität zu Göttingen
}

vorgelegt von

\section{Dieter Leßmann}

aus Dortmund

Göttingen, 1991 
Die vorliegende Dissertation ist in gedruckter Form veröffentlicht worden in Berichte des Forschungszentrums Waldökosysteme, Reihe A, Bd. 97, 1993. ISSN 0939-1347

D7

Referent: Prof. Dr. Ulrich Heitkamp

Korreferent: Prof. Dr. Matthias Schaefer

Tag der mündlichen Prüfung: 21. Juni 1991 


\section{Water acidification and running water biocoenoses in the Harz Mountains / Germany}

Keywords: acid rain, Harz Mountains, freshwater acidification, macrozoobenthos, fish, bioindication

\section{Summary}

From 1986 to 1989, as part of an interdisciplinary research project, limnological studies were carried out on the effects of water acidification on running water biocoenoses in the Harz Mountains, which were a region with high immissions of acidity ("acid rain"). For the investigations, 15 sampling sites in the epirhithral of six streams in the western part of the Harz Mountains were selected at which, due to different geological conditions, different intensities of water acidification occurred. The sites were comparable in terms of the morphological structure and the hydrological conditions and were free from direct anthropogenic influences. Six sampling sites in the Sösemulde at the non-acidified Alte Riefensbeek and the acidified Große Söse were subject to more intensive investigations between 1986 and 1989 than sites at other streams investigated in 1986 and 1987.

The study presents results of the physical and chemical investigations and in the main part the findings about the effects of freshwater acidification on the biocenotic structure of the macrozoobenthos comprising the groups Mollusca, Amphipoda, Ephemeroptera, Plecoptera and Coleoptera. The results were based on regular macrozoobenthos sampling and emergence recordings by permanent traps. The investigations included also the fish populations, which were studied by electrofishing.

The high intensity of air pollution led to high sulphate and nitrate concentrations in all water bodies of the study area. In many water bodies of the Harz Mountains with low concentrations of basic cations and being poor in electrolytes, this was associated with acidification. Analyses of extensive $\mathrm{pH}$ measurements enabled to divide the sampling sites into four hydrogeochemical groups with differences in the $\mathrm{pH}$ regime based on the intensity and frequency of acidification:

- non-acidified stream sections with only minor fluctuations in the hydrogen ion concentrations in the permanently neutral range,

- slightly acidic stream sections with average $\mathrm{pH}$ values in the neutral range and occasional periodic acidic events with $\mathrm{pH}$ decreases to around $\mathrm{pH} 5$,

- moderately acidic stream sections with average $\mathrm{pH}$ values in the slightly acidic range ( $\mathrm{pH} 5-6)$ and regular periodic $\mathrm{pH}$ decreases to $\mathrm{pH}$ values $<5$,

- heavily acidified stream sections with average $\mathrm{pH}$ values in the most acidic range $(\mathrm{pH}<5)$ that were permanently acidic or showed neutral $\mathrm{pH}$ values only for short periods.

The most important hydrochemical parameters that correlated with $\mathrm{pH}$ included the acid neutralizing capacity and the concentrations of aluminium, calcium, magnesium, cadmium and lead. Aluminium speciation analyses showed that the proportion of monomeric inorganic species decreased with increasing concentrations of dissolved organic carbon at the most acidic sites. A cluster analysis of the hydrochemical data resulted in a split of the sampling 
sites into two main groups with the non-acidified sites on one side and the acidified sites on the other.

A total of 76 species and higher taxa could be found for the macrozoobenthos, with the species-richest groups being the Plecoptera with 35 species and the Ephemeroptera with 19 species. For all groups, the number of species that occurred at a sampling site was highly dependent on the intensity of acidification. Gastropoda and Amphipoda could only be detected in the non-acidified water bodies. Ephemeroptera showed the greatest species decline with increasing acidification and were completely absent from the most acidic sites. Only Plecoptera and Coleoptera always inhabited also the heavily acidified stream sections.

Correspondingly, the autecological evaluation of the study results revealed very low to low acid tolerance for the Amphipoda, most of the Ephemeroptera and most of the species belonging to the Setipalpia within the order Plecoptera. Very high dominances of single Plecoptera species could occur at the most acidic sites, whereas the dominance structures of the moderately and weakly acidified sites progressively converged to those of the non-acidified sites, which showed the most balanced dominance structures. This was also reflected in an increase in the diversity indices.

With regard to population densities, the non-acidified sites usually showed higher total abundances than the acidified ones. However, mass developments of single Plecoptera species could occur in heavily acidified stream sections with population densities far exceeding those of non-acidified sites.

In the emergence studies, regardless of the degree of acidification, Ephemeroptera only accounted for a maximum of $20 \%$ to total emergence. While the Ephemeroptera already showed higher abundances in the non-acidified stream sections in spring, the order only appeared at acidified sites in summer and autumn. Total emergence reached the highest values at three of the four most acidic sites. No differences in emergence could be observed between moderate, weak, and non-acidified sites.

While the dominance structures in the strongly acidified stream sections were always determined by only a few eudominant species, a clear tendency towards more balanced structures could be observed with decreasing acidification. However, some species were able to achieve a very high dominance also at non-acidified sampling sites. The comparison of different years revealed only slight changes in the dominance structures of a certain sampling site.

Overall, there was a relatively high similarity in the dominant species composition within the various degrees of acidification. Correspondingly, a biocenotic similarity comparison revealed clearly separated biocenotic structures of acidified and non-acidified stream sections, with the heavily acidified sites showing the highest similarity among each other. Depending on the degree of acidification, characteristic biocenotic structures could be detected. For the diversity, the lowest values were calculated for the heavily acidified sections, whereas the differences among the groups of the moderately and weakly acidified stream sites were relatively small.

The investigations of the fish populations also revealed a strong dependency of fish inhabitation on the intensity of acidification. Bullhead (Cottus gobio) and brown trout (Salmo trutta fario) only occurred at non-acidified sampling sites. Normally structured fish populations could develop in higher densities only there. Bullheads were already absent from slightly acidic waters, and brown trout showed an abnormal age structure of the population under these conditions with low population densities due to the lack of young fish in most years. Moderately 
acidified waters only had a stock of a few larger, old brown trout. Fish were completely absent from heavily acidified stream sections.

By linking and analysing the biological and abiotic data, it was possible to prove that the observed differences in the structure of the benthic biocoenoses and in the fish populations were mainly due to the degree of water acidification, with both, increased hydrogen ion concentrations and, in some cases, increased concentrations of monomeric inorganic aluminium, being responsible for the observed biocenotic changes. The dependence of biocenotic effects on the degree of water acidification and the relatively characteristic emergence and benthic dominance structures within different degrees of water acidification can be used for a biological indication of the intensity of freshwater acidification caused by acid rain. 
Inhalt

Seite

1 Einleitung

3 Das Untersuchungsgebiet

3.1 Geographie 13

$\begin{array}{ll}3.2 \text { Geologie } & 15\end{array}$

$\begin{array}{ll}3.3 \text { Klima } & 16\end{array}$

$\begin{array}{ll}3.4 \text { Vegetation } & 16\end{array}$

4 Methoden 18

4.1 Kriterien für die Auswahl der Probestellen $\quad 18$

$\begin{array}{ll}4.2 \text { Abiotische Untersuchungen } & 18\end{array}$

$\begin{array}{ll}\text { 4.2.1 Physiographie } & 18\end{array}$

4.2.2 Physikalische Faktoren und Hydrochemie 19

$\begin{array}{ll}4.3 \text { Untersuchungen der Makrofauna } & 21\end{array}$

4.3.1 Beprobung und Bearbeitung des Makrozoobenthos 21

4.3.2 Erfassung und Bearbeitung der Emergenz 23

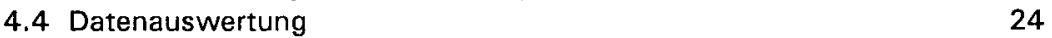

4.5 Untersuchungen der Fischfauna 28

5 Ergebnisse $\quad 30$

5.1 Die Probestellen und ihre abiotischen Charakteristika 30

5.1.1 Geographie, Geologie, Pedologie 30

5.1.1.1 Das Hauptuntersuchungsgebiet Sösemulde
mit Großer Söse und Alter Riefensbeek

5.1.1.2 Großer Mollenbach und Große Schacht 36

5.1.1.3 Große Bode und Warme Bode 36

5.1.1.4 Lange Bramke $\quad 37$

$\begin{array}{ll}5.1 .1 .5 \text { Varleybach } & 37\end{array}$

5.1.2 Wetter und Schadstoffdeposition im Untersuchungszeitraum 37

$\begin{array}{ll}\text { 5.1.2.1 Wetter } & 37\end{array}$

5.1.2.2 Atmosphärische Schadstoffdeposition 40 
Seite

5.1.3 Hydrographie $\quad 42$

5.1.3.1 Abfluß $\quad 42$

$\begin{array}{ll}5.1 .3 .2 \text { Gefälle } & 47\end{array}$

5.1.3.3 Tiefe und Breite $\quad 48$

5.1.3.4 Fließgeschwindigkeit und Morphologie 50

5.1.3.5 Choriotopstruktur $\quad 52$

5.1.4 Gewässerrandvegetation, Primärproduktion und allochthone Nährstoffversorgung $\quad 54$

5.1.5 Physikalisch-chemische Untersuchungsergebnisse 58

5.1.5.1 Wasserstoffionenkonzentration 58

$\begin{array}{lll}5.1 .5 .2 & \text { Wassertemperatur } & 69\end{array}$

$\begin{array}{lll}5.1 .5 .3 & \text { Sauerstoff } & 70\end{array}$

5.1.5.4 Gelöster organischer Kohlenstoff (DOC) 70

$\begin{array}{ll}\text { 5.1.5.5 Elektrische Leitfähigkeit } & 72\end{array}$

$\begin{array}{ll}\text { 5.1.5.6 Säurekapazität und Calcium } & 74\end{array}$

5.1.5.7 Magnesium $\quad 75$

$\begin{array}{ll}5.1 .5 .8 & \text { Sulfat und Nitrat }\end{array}$

$\begin{array}{ll}\text { 5.1.5.9 Ammonium } & 79\end{array}$

$\begin{array}{ll}\text { 5.1.5.10 Aluminium } & 79\end{array}$

5.1.5.11 Zink, Cadmium, Blei 83

5.1.6 Zusammenfassende Betrachtung der Hydrochemie der Probestellen und der Gewässersysteme $\quad 86$

5.1.6.1 Vergleich der Probestellen 86

5.1.6.2 Hydrochemie der Gewässersysteme 90

5.2 Artenspektrum der Gewässerbiozönosen 92

5.3 Autökologische Ergebnisse zur Versauerungstoleranz $\quad 104$

$\begin{array}{ll}\text { 5.3.1 Mollusca } & 106\end{array}$

$\begin{array}{ll}\text { 5.3.2 Amphipoda } & 106\end{array}$

$\begin{array}{ll}\text { 5.3.3 Ephemeroptera } & 109\end{array}$

$\begin{array}{ll}\text { 5.3.4 Plecoptera } & 114\end{array}$

$\begin{array}{ll}5.3 .5 \text { Coleoptera } & 120\end{array}$

5.4 Benthosuntersuchungen $\quad 123$

5.4.1 Benthos der Hauptuntersuchungsbäche $\quad 124$

$\begin{array}{ll}\text { 5.4.1.1 Besiedlungsdichten } & 124\end{array}$

5.4.1.2 Dominanzstrukturen $\quad 130$

5.4.1.3 Zusammenfassender Vergleich der Probestellen 136

5.4.2 Benthos der Haupt- und Nebenuntersuchungsbäche im Vergleich 
5.5 Emergenzerfassung

5.5.1 Emergenz der Hauptuntersuchungsbäche 143

5.5.1.1 Artenzahlen 143

5.5.1.2 Emergenzdichten $\quad 143$

5.5.1.3 Dominanzstrukturen $\quad 145$

5.5.1.4 Zeitlicher Ablauf $\quad 151$

5.5.1.5 Biozönotischer Ähnlichkeitsvergleich 155

5.5.2 Emergenz der Nebenuntersuchungsbäche 158

$\begin{array}{ll}\text { 5.5.3 Geschlechterverhältnis } & 167\end{array}$

5.5.4 Biozönotischer Ähnlichkeitsvergleich 168

$\begin{array}{ll}5.6 \text { Ichthyozönosen } & 175\end{array}$

5.6.1 Vorkommende Arten 175

5.6.2 Groppenpopulationen 176

5.6.3 Bachforellenpopulationen der Hauptuntersuchungsbäche $\quad 178$

$\begin{array}{ll}\text { 5.6.3.1 Populationsstruktur } & 179\end{array}$

$\begin{array}{ll}\text { 5.6.3.2 Besiedlungsdichte } & 185\end{array}$

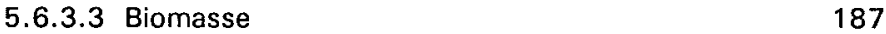

$\begin{array}{ll}\text { 5.6.3.4 Korpulenzfaktor } & 187\end{array}$

5.6.4 Bachforellenpopulationen der Haupt- und Nebenuntersuchungsbäche im Vergleich $\quad 187$

$\begin{array}{ll}\text { 5.6.4.1 Populationsstruktur } & 187\end{array}$

5.6.4.2 Besiedlungsdichte und Biomasse 189

$\begin{array}{ll}\text { 5.6.4.3 Korpulenzfaktor } & 190\end{array}$

6 Diskussion $\quad 197$

6.1 Bedeutung der abiotischen Faktoren für die Zusammensetzung der Biozönosen

6.2 Auswirkungen der Versauerung auf die Biozönosen 205

6.3 Möglichkeiten und Wege zu einer biologischen Indikation der Gewässerversauerung

7 Zusammenfassung

8 Literatur 


\section{Einleitung}

Obwohl sich die wissenschaftliche Forschung mit der Gewässerversauerung erst seit zwei Jahrzehnten befaßt, konnten ihre Ursprünge in vielen Gebieten bereits auf die Anfänge unseres Jahrhunderts zurückdatiert werden. Großflächig bemerkbar machte sich die Versauerung zuerst in Nordamerika und Skandinavien, wo der Verlust wirtschaftlich wertvoller Fischbestände das Interesse an der Problematik weckte und in den 70er Jahren größere Forschungsaktivitäten auslöste. Die Ergebnisse dieser zahlreichen Untersuchungen, die sich vor allem auf Seen erstreckten, deckten die schweren Folgen der übermäßigen Luftschadstoffimmissionen für die Gewässerökosysteme auf (Zusammenfassungen u.a. durch OVERREIN et al. 1980, COWLING 1980, DRABLOS \& TOLLAN 1980, HAINES 1981, D'ITRI 1982, MASON \& SEIP 1985).

Die dadurch in Gang gesetzte wissenschaftliche Diskussion führte zur Suche nach von der Gewässerversauerung betroffenen Regionen in Mitteleuropa. Eine Bestandsaufnahme für die Bundesrepublik Deutschland verdeutlichte, welche Dimension diese Umweltzerstörung auch hier bereits erreicht hatte (SCHOEN et al. 1983, UBA 1984). Durch die Erschließung der weltweiten Forschungsergebnisse (LENHART \& STEINBERG 1984a) wurde eine Basis für die weiteren nationalen Forschungen erstellt.

Im Harz gibt es auf basenarmen Gesteinen sehr saure Gewässer, die lange Zeit für von Natur aus sauer gehalten wurden. Erst das Auftreten der sogenannten neuartigen Waldschäden und die dafür verantwortlich gemachten hohen Einträge von Schadstoffen aus der Luft ließen deutlich werden, daß eine Versauerung stattgefunden haben muß.

Bei faunistischen Untersuchungen wurden im Jahr $1968 \mathrm{im}$ oberen und mittleren Bereich der Oder pH-Werte bis weit unter 4,0 gemessen (GRASHOF 1972). Anfang der 70er Jahre wiesen bereits alle untersuchten Quellabflüsse auf AckerBruchberg-Quarzit pH-Werte $<5$ auf (ALICKE 1974). Untersuchungen an der Großen Romke Mitte der 70er Jahre erbrachten $\mathrm{pH}$-Werte zwischen 4 und 5 (BROCK \& BLUM 1977). Zum diesem Zeitpunkt war es jedoch noch zu früh, als daß die Tragweite der Messungen und der tatsächliche Umfang der Auswirkungen der hohen Wasserstoffionenkonzentrationen auf die Ökosysteme im Harz erkannt worden wären. Untersuchungen an der im Oberlauf stark versauerten Sieber fanden dagegen schon zu einer Zeit statt, wo die Zusammenhänge 
zwischen Luftschadstoffdepositionen, Waldsterben und Gewässerversauerung deutlicher wurden (HEITKAMP et al. 1985). Die Gewässerversauerung hat heute ein Ausmaß erreicht, daß bereits die Gewässer eines Fünftel der niedersächsischen Harzfläche keinen Fischbestand mehr aufweisen (LESSMANN \& HEITKAMP 1990).

Ziel des Forschungsvorhabens war es, in interdisziplinärer Zusammenarbeit die Auswirkungen von Luftschadstoffeinträgen auf Fließgewässerbiozönosen im Harz durch eine umfassende biologische, chemische und hydrologische Charakterisierung zu ermitteln (vgl. Kap. 2). Durch vergleichende Untersuchungen an Gewässern ähnlicher Lage und Struktur, wie sie erstmals in Skandinavien im Zusammenhang mit Forschungen zur Gewässerversauerung erfolgreich durchgeführt wurden (OVERREIN et al. 1980), soliten das Ausmaß der Versauerung unter verschiedenen geologischen Bedingungen bestimmt, die Reaktionen der Biozönosen auf unterschiedliche Belastungen ermittelt sowie die für Veränderungen verantwortlich zu machenden und die Strukturen der Biozönosen bestimmenden Faktoren identifiziert werden. Anhand der Ergebnisse sollten Möglichkeiten und Wege einer biologischen Indikation der Gewässerversauerung geprüft werden.

In die limnologischen Untersuchungen einbezogen wurden alle wichtigen Gruppen der Makro- und Mesofauna, die Fische und als wichtigste Primärproduzenten die Diatomeen (HEITKAMP \& LESSMANN 1990, HEITKAMP et al. 1991). Die vorliegende Arbeit hat die Ergebnisse der Untersuchungen an den Makrofaunagruppen der Mollusca, Amphipoda, Ephemeroptera, Plecoptera und Coleoptera sowie die Ergebnisse der fischereikundlichen Untersuchungen zum Inhalt. Neben Benthosbeprobungen wurde auch die Emergenz erfaßt. Die systematisch schwierige Gruppe der Diptera bleibt einer eigenen Bearbeitung vorbehalten (ROMMELMANN in Vorb.). Ebenfalls eigenständig bearbeitet wurde die artenreiche Gruppe der Trichoptera (RÜDDENKLAU 1989, RÜDDENKLAU 1990, RÜDDENKLAU 1991a, RÜDDENKLAU 1991b). Die Freilandbearbeitungen der Makroinvertebraten wurden durch laborexperimentelle Untersuchungen ergänzt (AVERMANN 1990a, AVERMANN 1990b). Weitere Untersuchungen befassen sich mit der Fauna des hyporheischen Interstitials und der Moospolster (WULFHORST 1991). Eine erste Untersuchungsphase der Diatomeen ist abgeschlossen (CORING 1988, CORING 1989, CORING 1990). 
Das Forschungsvorhaben wurde mit Mitteln des Bundesministers für Forschung und Technologie unter dem Förderkennzeichen 0339069B sowie in Teilbereichen auch mit Mitteln des Bundesministers für Umwelt, Naturschutz und Reaktorsicherheit im Rahmen des "Monitoringprogramms für versauerte Gewässer durch Luftschadstoffe in der Bundesrepublik Deutschland im Rahmen der ECE" (UFO-Plan 10204 362) gefördert. 


\section{Zur "Fallstudie Harz"}

Der Harz gehört seit vielen Jahren zu den durch Luftschadstoffe mit am höchsten belasteten Gebieten Mitteleuropas. Die Folgen sind zum einem das seit etwa 10 Jahren großflächig sichtbare Waldsterben und die erst vor wenigen Jahren ins Bewußtsein der Öffentlichkeit gerückte Versauerung von Bächen und Seen. Aufbauend auf die bisherige Waldschadensforschung im Harz im Einzugsgebiet der Langen Bramke (HAUHS 1985a-c) sowie auf Beobachtungen der Gewässerversauerung am Harzfluß Sieber (LESSMANN 1983, PIEHL 1983, HEITKAMP et al. 1985) und auf hydrogeochemische Untersuchungen von Quellen in der Sösemulde (HEINRICHS et al. 1986) fanden sich 1985 Geochemiker, Forstbodenkundler, Landschaftsökologen, Limnogeologen und Limnologen unter der Federführung des Forschungszentrums Waldökosysteme der Universität Göttingen zusammen, um ein interdisziplinäres Forschungsvorhaben unter dem Titel "Fallstudie Harz: Schadstoffbelastung, Reaktion der Ökosphäre und Wasserqualität" zu planen.

Aufgrund der Ergebnisse von Voruntersuchungen wurde für die Forschungsarbeiten das Einzugsgebiet der Sösetalsperre ausgewählt, die eine wichtige Rolle bei der Trinkwasserversorgung des südniedersächsischen Raums spielt. Seit Oktober 1986 sind vom Bundesminister für Forschung und Technologie im Teilprogramm "Umweltforschung", Aktivität "Bodenbelastung und Wasserhaushalt", Teilaktivität "Belastungen der Oberflächengewässer / des Grundwassers" fünf Vorhaben des Verbundprojektes gefördert worden:

- "Untersuchungen der Seesedimente in der Sösetalsperre zur Rekonstruktion der Entwicklung der Belastungen in den letzten 53 Jahren sowie der letzten 250 Jahre im Oderteich", Institut für Geologie und Dynamik der Lithosphäre der Universität Göttingen, Leitung: Prof. Dr. Jürgen Schneider

- "Schwermetallbilanz aus Immission und geogenem Anteil im Einzugsgebiet der Sösetalsperre im Harz", Bundesanstalt für Geowissenschaften und Rohstoffe, Hannover, Leitung: Dr. Uirich Siewers

- "Lage und Vorschreiten der Versauerungsfront an Beispielen der Sösemulde", Institut für Bodenkunde und Waldernährung der Universität Göttingen, Leitung: Prof. Dr. Bernhard Ulrich, und Fachbereich Stadt- und Landschaftsplanung der Gesamthochschule Kassel, Leitung: Prof. Dr. Robert Mayer 


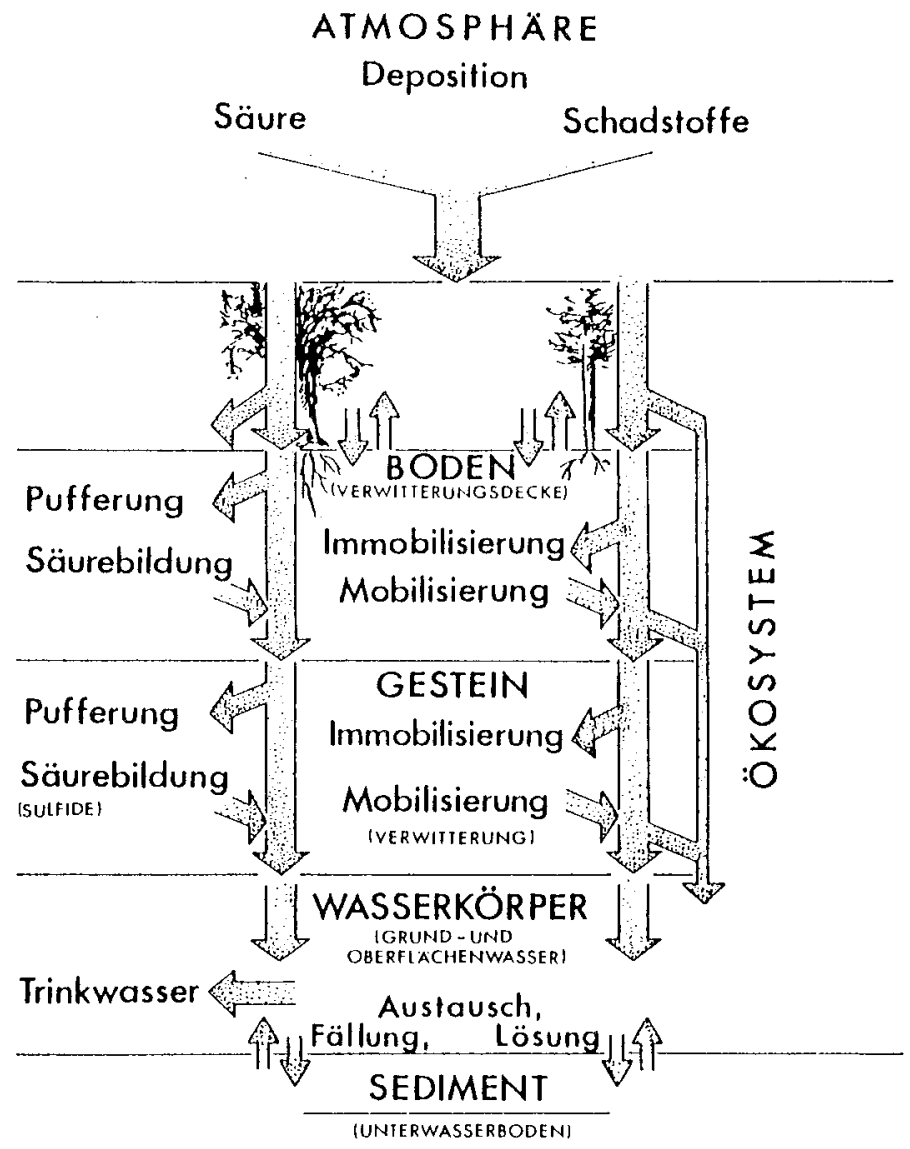

Abb. 1: Schadstoffwege im Ökosystem (aus MATSCHULLAT 1989) 
- "Auswirkungen der Fließgewässerversauerung auf die Bergbachbiozönosen", II. Zoologisches Institut der Universität Göttingen, Leitung: Prof. Dr. Ulrich Heitkamp

- "Stabile und metastabile Mineralphasen im Sickerwasserleiter", Geochemisches Institut der Universität Göttingen, Leitung: Prof. Dr. Karl Heinz Wedepohl und Dr. Hartmut Heinrichs

Die gemeinsame Bearbeitung der Frage des Weges der Schadstoffe durch das Ökosystem (Abb. 1) und der Folgen, die sich daraus für die einzelnen Ökosystemkompartimente ergeben, erbrachte eine Reihe von Erkenntnissen zu Zusammenhängen im System, von der atmosphärischen Deposition über die Zwischenlagerung in den Böden, dem Eintrag in die Gewässer und dem Weg in den Fließgewässern bis zur Ablagerung im Stausee sowie den daraus resultierenden Folgen für die Gewässerbiozönosen. Im einzelnen wurden im abiotischen Teil der Forschungen unter anderem die Gesamtdeposition im Freiland und im Bestand gemessen (SIEWERS \& ROOSTAI 1990), der Tiefengradient der Bodenversauerung, die im Boden wirksamen Pufferungsprozesse und die Mobilisierung vonSchwermetallen in versauerten Böden untersucht (ULRICH \& MALESSA 1989, MALESSA \& ULRICH 1989, ANDREAE \& MAYER 1989, MALESSA \& ANDREAE 1990), durch geochemische Untersuchungen geogene Stoffbelastungen oder Belastungen durch alte Bergbauaktivitäten ausgeschlossen bzw. quantifiziert, die Hydrochemie der Gewässer des Einzugsgebietes des Sösestausees kartiert und die Schadstoffbelastungen der Seesedimente ermittelt (Abb. 2)(MATSCHULLAT et al. 1987, ROOSTAI 1987, MATSCHULLAT 1989, MATSCHULLAT et al. 1990, BLAUME 1990, RATMEYER 1990, SIEWERS \& ROOSTAI 1990). Die biologischen Untersuchungen erstreckten sich in ausgewählten Fließgewässern auf Diatomeen, Makrozoobenthos, Fischfauna und die Fauna des hyporheischen Interstitials und der Moospolster (vgl. Kap. 1). Die biologischen Seebearbeitungen umfaßten Zooplankton und Zoobenthos (WILLERS 1988, HEITKAMP \& WILLERS 1990). 


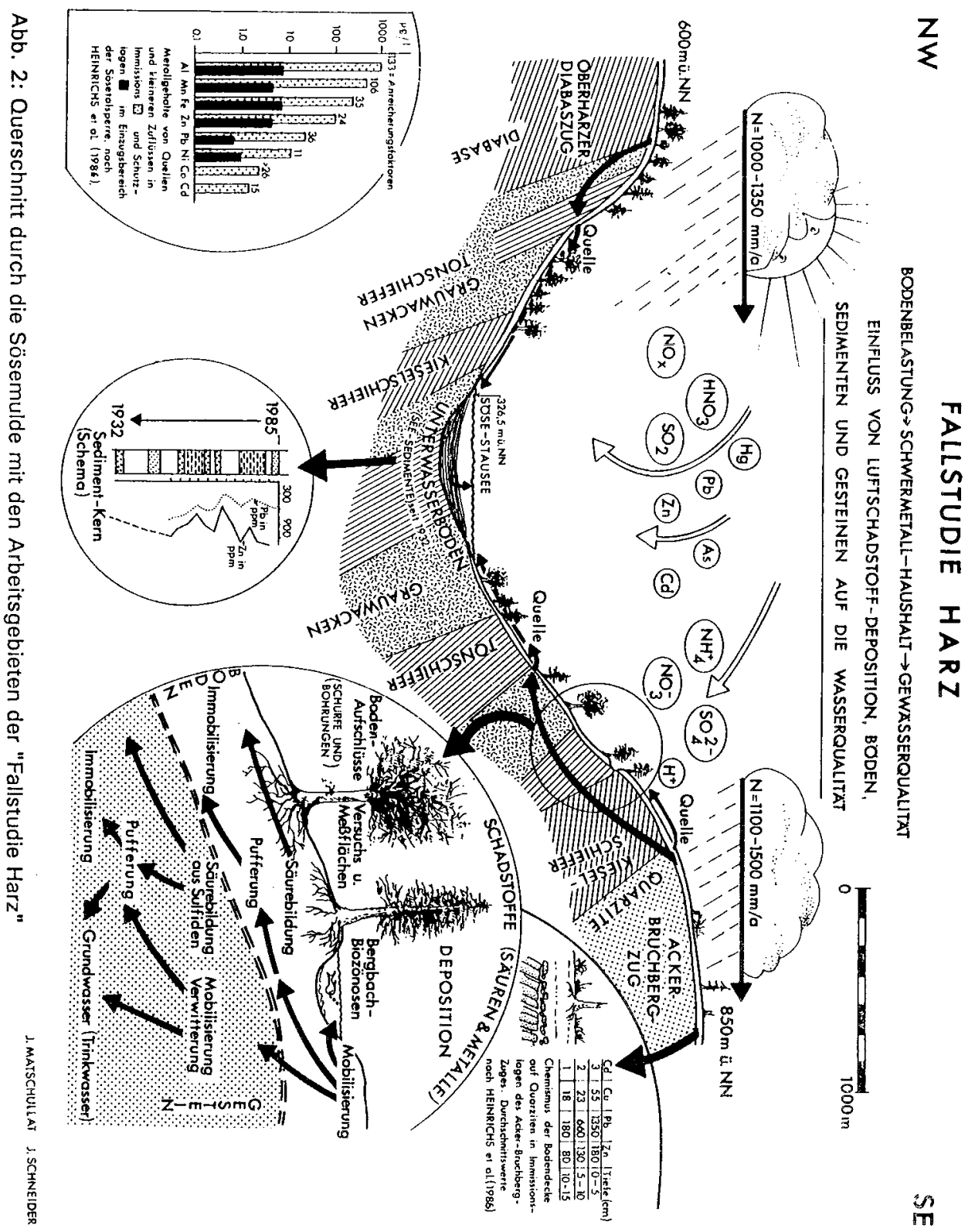




\section{Das Untersuchungsgebiet}

\subsection{Geographie}

Der in den Bundesländern Niedersachsen, Sachsen-Anhalt und Thüringen liegende Harz stellt ein etwa $90 \mathrm{~km}$ langes und $30 \mathrm{~km}$ breites Massiv mit einer Fläche von $2226 \mathrm{~km}^{2}$ dar, das die umgebende Landschaft weit überragt (MEYNEN et al. $1953 \mathrm{ff}$., GELLERT 1958, SINGER \& FLIEDNER 1970). Die schroffe Abgrenzung des Harzes wird insbesondere in den $300-400 \mathrm{~m}$ steilen Randstufen im Nordwesten deutlich, wohingegen im Süden und Südwesten ein allmählicherer Anstieg zu verzeichnen ist. An diese unterschiedlich stark ausgeprägten Randstufen schließen sich Hochflächen an, die von einzelnen Bergen und Bergrücken überragt werden. Die höchsten Erhebungen des Harzes sind Brocken (1142 m), Wurmberg $(971 \mathrm{~m})$, Bruchberg $(928 \mathrm{~m})$ und Achtermann $(926 \mathrm{~m})$.

Größere Siedlungen finden sich vor altem auf den Hochflächen. Im Gegensatz zum landwirtschaftlich genutzten Umland ist der Harz von ausgedehnten Wäldern bedeckt, zwischen denen sich stellenweise Hochmoore erstrecken.

Ausgangspunkte der zahlreichen Flüsse sind neben den Hochflächen die Berghänge und Bergrücken. Die Hauptflußsysteme auf niedersächsischem Gebiet sind - von Nord über West nach Süd - die der Ecker, Radau, Oker, Grane, Innerste, Söse, Sieber, Oder, Zorge und Bode. Durch die umfangreiche Anlage von Wassergräben, Teichen und Talsperren unterliegen diese Flußsysteme auf längeren Strecken einer weitgehenden anthropogenen Beeinflussung. So hat das im niedersächsischen Harzteil existierende System von Hang- und Sammelgräben eine Länge von $240 \mathrm{~km}$ (HAASE et al. 1970). Die intensive Einflußnahme des Menschen auf das Gebiet begann mit dem Bergbau im Mittelalter, als der Harz von Menschen besiedelt wurde und Industrieanlagen entstanden. Der 17141721 erbaute Oderteich ist die älteste erhaltene Talsperre Deutschlands. Von den größeren Flüssen wird bisher nur die Sieber nicht von einer Talsperre aufgestaut. 


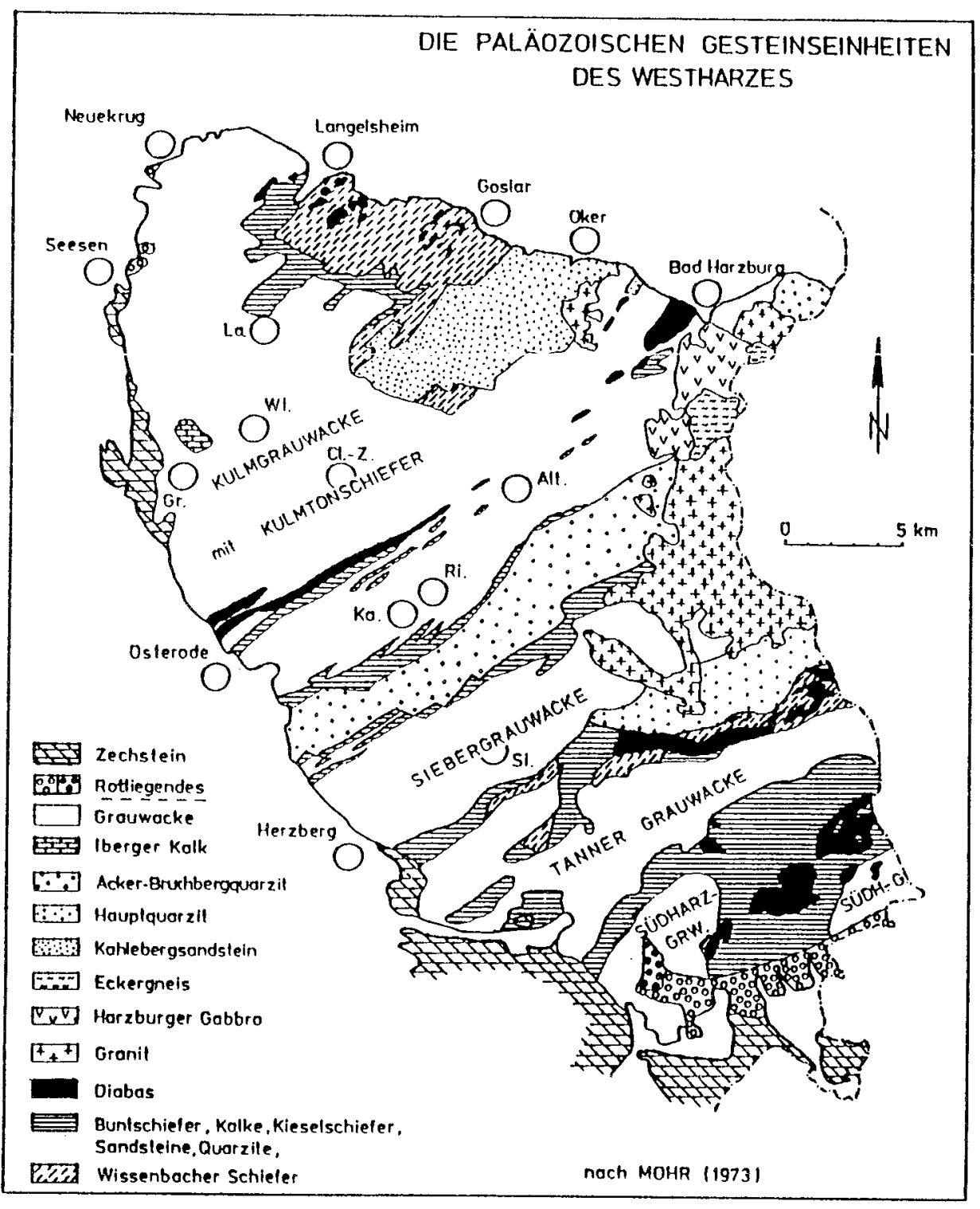

Abb. 3: Übersichtskarte zur Geologie des in Niedersachsen gelegenen Harzteils (aus ALICKE 1974) 


\subsection{Geologie}

Der Harz stellt sowohl groß- als auch kleinräumig gesehen ein kompliziertes Horstgebirge dar. Er ist ein Teil des Rhenoherzynikums, der nordwestlichen Einheit des mitteleuropäischen Varistikums (MOHR 1984, WAGENBRETH \& STEINER 1989).

Das varistische Gebirge ist an der Wende Devon/Karbon entstanden und erstreckte sich von den Ardennen über Schwarzwald, Spessart, Rheinisches Schiefergebirge und Harz nach Osten. Im Verlauf der Orogenese wurden paläozoische Sedimente angehoben und gefaltet. Dabei kam es zu intrusionen von Magmatiten, worauf u.a. das heutige Brockenmassiv zurückzuführen ist. Die Faltung des Harzes ist NW-vergent, wobei das Streichen im Inneren des Gebirges in SW-NO-Richtung verläuft. Die Harz-Tektonik wird als einphasige, von Südosten nach Nordwesten fortschreitende Deformation gedeutet.

Zeitgleich zur Auffaltung kam es zu einer starken Erosion. Bereits im Oberkarbon überlagerten im Südost-Harz in einem intramontanen Senkungsbecken Sedimente das varistische Gebirge durch Molasse-ähnliche Schuttmassen mit Kohlelagen. Gleichzeitig traten saure Vulkanite auf. Synorogene rotliegende Sedimente füllten das während des Devons angelegte Relief auf. Die roten Konglomerate, Sandsteine und Schluffsteine im lifelder und Meisdorfer Becken sind hierfür Beispiele.

Im Perm, zum Ausgang des Paläozoikums, war der Harz durch das vorherrschende aride Klima fast vollständig abgetragen. Nur der Quarzit-Zug, die Granite und einige Porphyr-Stöcke ragten noch über das transgredierte Zechstein-Meer hinaus. Bis zum beginnenden Lias legten sich diskordant Schichten des Zechsteins und der Trias über das varistisch gefaltete Gestein.

An der Wende Jura/Kreide wurde die Harz-Scholle angehoben und anschließend wiederum völlig erodiert. Zu Beginn einer erneuten Hebungsphase im Tertiär, die das heutige Mittelgebirge schuf, lag das paläozoische Grundgebirge frei. Im Pleistozän erfolgte die jüngste Hebung des Harzes. Durch die anhaltende Erosion ist die alte varistische Morphologie wieder hervorgehoben worden. Während der einzelnen Hebungsphasen seit dem Jura kam es zu einer deutlichen Kippung der Scholle, die von Norden nach Süden um einige hundert Meter geneigt ist. Noch heute stellt der Harz eine gravimetrische Schwereanomalie dar. 
Fast alle Gesteine im Harz sind Festgesteine ohne größeres Porenvolumen oder größere Permeabilität. Es ist ein Kluftgrundwasserleiter ausgebildet, wobei es zu keinem großflächigen Kontakt von Wasser und Gestein kommt und sich nur wenige Stoffe lösen können (ALICKE 1974).

Die Pedologie des Harzes wird vor allem durch flache bis mittlere Braunerden bestimmt (HÖVERMANN 1953).

\subsection{Klima}

Das Klima ist bei vorherrschenden westlichen Wirdrichtungen ein subatlantisches Gebirgsklima. Aufgrund von Höhe und Lage sind das ganze Jahr hindurch reichliche Niederschläge zu verzeichnen. Die jährliche Niederschlagssumme steigt im Westen von $900 \mathrm{~mm}$ am Harzrand über $1300 \mathrm{~mm}$ auf den Hochflächen bis auf $1640 \mathrm{~mm}$ am Brocken an. Die Niederschläge sind relativ gleichmäßig über das Jahr verteilt (DWD 1986-1989).

Die niedrigen Durchschnittstemperaturen der Wintermonate (Dezember bis Februar $\left.<0{ }^{\circ} \mathrm{C}\right)$ führen häufig zu einer Speicherung der Niederschläge in meterdicken Schneedecken. Die mittlere Zahl der Tage mit Schneedecken beträgt in den Hochlagen 80 bis 120 bei einer Schneedeckenperiode von 6 Monaten (MEYNEN et al. $1953 \mathrm{ff}$.). Die Bildung von Schneedecken wird im Winter häufig durch Tauperioden unterbrochen, die in den Flüssen zu Hochwässern führen. Bodenfrost tritt nur auf, wenn schneefreier Boden tiefen Temperaturen ausgesetzt ist. Der Höhenlage entsprechend vermindern sich die Jahresdurchschnittstemperaturen bis auf $2,4^{\circ} \mathrm{C}$ am Brocken. Der Harz hebt sich dadurch auch klimatisch aus seiner Umgebung ab.

\subsection{Vegetation}

Die Verbreitung von Hochmooren in den Höhenlagen mit den sie großflächig umgebenden versumpfenden Fichtenwäldern ist im Zusammenhang mit der Morphologie und den hohen Niederschlagsmengen zu sehen. Ansonsten bestimmen Fichtenforste weitestgehend das Bild bis hinab in die mittleren Lagen, wobei die untere natürliche Verbreitungsgrenze der Fichte bei $750-850$ $m$ gelegen haben dürfte. Nach oben hin wird die Baumgrenze am Brocken 
bereits erreicht. Im unteren Bereich der montanen Stufe sind insbesondere nach Süden hin zunehmend Buchenwälder und im submontanen Bereich Buchenmischwälder zu finden (ELLENBERG 1986).

Auf den besiedelten Hochflächen erstrecken sich ausgedehnte Grünflächen. Ackerbau wird nur in geringem Umfang betrieben. In den unteren Lagen ziehen sich Wiesenflächen entlang der Flußläufe in den Harz hinein. Sonst bilden die Wälder relativ lückenlose Bestände, soweit sie nicht durch das Waldsterben aufgelockert sind.

Historisch gesehen wurde die Vegetation des Harzes bis ins ausgehende 15. Jahrhundert durch Buchenmischwälder geprägt, die aufgrund des großen Holzbedarfs des Bergbaus um 1700 vollständig abgehoizt waren und später durch die schnellwüchsigen Fichten ersetzt wurden (WILLUTZKI 1962).

Aufgrund der nährstoffarmen Böden und des feuchtkühlen Klimas gehört der Harz mit weniger als 350 Pflanzenarten pro $100 \mathrm{~km}^{2} \mathrm{zu}$ den floristisch ärmsten Gegenden Südniedersachsens (MEINEKE 1984 zit. nach RÜDDENKLAU 1989). 


\section{Methoden}

4.1 Kriterien für die Auswahl der Probestellen

Für die von April 1986 bis April 1989 durchgeführten Untersuchungen wurden 15 Probestellen an sechs über den Oberharz verstreut liegenden Bächen ausgewählt. Die Festlegung der Untersuchungsgewässer und der Probestellen erfolgte auf der Grundlage von eigenen Voruntersuchungen sowie Untersuchungen des Geochemischen Instituts (HEINRICHS et al. 1986), des Instituts für Bodenkunde und Waldernährung der Universität Göttingen (HAUHS 1985a-c) und der Harzwasserwerke in enger Abstimmung mit den anderen an den Gesamtforschungsprojekten beteiligten Arbeitsgruppen.

Die Schwerpunktuntersuchungen im Rahmen der "Fallstudie Harz" wurden an den in der Sösemulde liegenden Bächen Alte Riefensbeek und Große Söse durchgeführt. Die Bearbeitung der Nebenuntersuchungsbäche, die im Rahmen des ECE-Monitoringprogramms "Gewässerversauerung in der Bundesrepubik Deutschland" von April 1986 bis Dezember 1987 bearbeitet wurden, erfolgte zusammen mit dem Zentrallabor der Harzwasserwerke, das darüber hinaus auch in die Arbeiten an den Hauptuntersuchungsbächen einbezogen war.

Kriterien für die Probestellenauswaht waren die Berücksichtigung als sensitiv bzw. weniger sensitiv gegenüber der Gewässerversauerung bekannter, für den Harz repräsentativer geologischer Bereiche, der Ausschluß direkter anthropogener Belastungen und eine möglichst gute Zugänglichkeit zu jeder Jahreszeit. Darüber hinaus sollten jeweils mehrere der zu untersuchenden Gewässerabschnitte von Höhenlage, Gefälle, Quellentfernung und hydromorphologischer Struktur vergleichbare Bedingungen aufweisen.

\subsection{Abiotische Untersuchungen}

\subsubsection{Physiographie}

Die Daten für Höhenlage, Quellentfernung und Gefälle an den Probestellen wurden anhand der Topographischen Karten 1 : 25.000 des Niedersächsischen Landesverwaltungsamtes, Blätter 4127, 4128, 4227 - 4229 ermittelt. Zur Beschreibung der Abflußverhältnisse wurden die Daten der Pegelschreiber an Söse, Schacht, Langer Bramke und Varleybach von den Harzwasserwerken zur Verfügung gestellt. Daneben wurde an den Hauptuntersuchungsbächen auf die 
hydrologischen Untersuchungen von SIEBERT (1988) und den Bericht von SIEBERT \& VIERHUFF (1989) zurückgegriffen.

Breite und Tiefe der Gewässer wurden bei mittlerem Abfluß gemessen. Vor Ort kartiert bzw. erfaßt wurden auch Längsentwicklung, Querprofil, Einzugsgebietsund Gewässerrandvegetation sowie Beschattung. Der Auswertung der Choriotopstruktur liegen sowohl Kartierungen als auch wiederholte Abschätzungen der Substratverhältnisse im Freiland zugrunde. Für die Angaben zu submerser Vegetation, allochthonen Nährstoffen, Geschiebeführung und zur Charakterisierung der Fließgeschwindigkeit wurden die bei allen Freilandarbeiten festgehaltenen Beobachtungen herangezogen.

\subsubsection{Physikalische Faktoren und Hydrochemie}

Die hydrochemischen Untersuchungen erfolgten in Kooperation mit dem Zentrallabor der Harzwasserwerke in Langelsheim (Dr. Peter Groth, Ralf Trapphoff), der Bundesanstalt für Geowissenschaften und Rohstoffe in Hannover (Dr. Ulrich Siewers, Abdul Hanan Roostai) und dem Institut für Bodenkunde und Waldernährung der Universität Göttingen (Dr. Gabi Dietze). Die Empfehlungen des Umweltbundesamtes zur Überwachung versauerter Gewässer wurden berücksichtigt (UBA 1987).

Durch eigene Messungen wurden 1986 in zwei- bis dreiwöchigen, 1987 und 1988 in einwöchigen und teilweise auch kürzeren Abständen an physikochemischen Faktoren $\mathrm{pH}$, Leitfähigkeit, Wasser- und Lufttemperatur gemessen, wobei 1988 nur noch die Hauptuntersuchungsbäche in das dichte Meßprogramm einbezogen waren. Die pH-Messungen erfolgten 1986 mit einer Gel-Elektrode in der fließenden Welle. Alle Messungen seit Beginn des Jahres 1987 wurden mit einer Glas-Elektrode in einem Becherglas durchgeführt. Vergleichsmessungen zeigten, daß mit der Gel-Elektrode im sauren Bereich in der Regel bei größeren Schwankungen zwischen wiederholten Messungen mehrere Zehntel pH-Einheiten weniger gemessen werden als mit der zuverlässigeren Glas-Elektrode. Für die statistischen Auswertungen der pH-Messungen wurden deshalb nur die Daten der Jahre 1987 - 1989 zugrunde gelegt.

Exakte Messungen der Fließgeschwindigkeit wurden bei allen faunistischen Probenahmen an den Probepunkten der Teilprobenahmen mit einem Tauchstab 
nach JENS (1968) (Fa. Hydrobios, Kiel) vorgenommen, der die mittlere Fließgeschwindigkeit der Wassersäule am Meßpunkt anzeigt.

Die Probenahmen für die Laboranalytik in PE- und Glasgefäßen mit Filtration der Proben im Labor durch 0,45 $\mu \mathrm{m}$-Filter sowie die Analytik selbst wurden 1986 und 1987 vom Zentrallabor der Harzwasserwerke in überwiegend einwöchigen Abständen durchgeführt (Tab. 1). Von März 1988 bis April 1989 wurden die Proben durch Mitarbeiter des II. Zoologischen Instituts genommen, nach Sofortfiltration durch $0,45 \mu \mathrm{m}$-Filter in säuregespülte PTFE-Flaschen überführt und in der BGR analysiert (s. auch SIEWERS \& ROOSTAI 1990). Die Veröffentlichung der Einzelergebnisse der Laboranaiysen bleibt den Harzwasserwerken bzw. der BGR vorbehalten.

Tab. 1: Übersicht zu den Meß- und Analysemethoden

eigene Messungen

Harzwasserwerke

BGR Hannover

\begin{tabular}{c|ccc}
\hline $\begin{array}{c}\text { Fließ- } \\
\text { geschwindigkeit } \\
\begin{array}{c}\text { Wasser- } \\
\text { temperatur }\end{array}\end{array}$ & $\begin{array}{c}\text { Tauchstab } \\
\text { nach JENS } \\
\text { WTW LF 91, } \\
\text { Min-Max- } \\
\text { Thermometer } \\
\text { WTW pH 91 mit } \\
\text { Ingold 405-KN7 } \\
\text { WTW Oxi 91 }\end{array}$ \\
Sauerstoff \\
DOC \\
el. Leitfähigkeit \\
Säurekapazität \\
Calcium \\
$\begin{array}{c}\text { Magnesium } \\
\text { Sulfat } \\
\text { Nitrat }\end{array}$
\end{tabular}


Analysen zur Ermittlung der Konzentrationen der monomeren anorganischen und monomeren organischen Bindungsformen des Aluminiums wurden im Institut für Bodenkunde und Waldernährung der Universität Göttingen für alle Gewässer außer der Langen Bramke zwischen April 1988 und April 1989 vorgenommen, wobei für jede Probestelle zwischen 7 und $14 \mathrm{im}$ Freiland durch $0,45 \mu \mathrm{m}$ filtrierte und in PE-Flaschen transportierte Proben bearbeitet wurden. Die Gesamtheit der monomeren Species wurde mit Hilfe der photometrischen Methode Eriochrome cyanine $\mathbf{R}$ und Cetyltrimethylammoniumbromid im Fließinjektionsverfahren (FIA-ECR/CTA) gemessen. Die untere Nachweisgrenze liegt bei $10 \mu \mathrm{g} / \mathrm{l}$. Durch Kationenaustausch wurde die organisch gebundene Fraktion bestimmt, und die monomere anorganische Fraktion durch Differenzbildung aus den Konzentrationen an gesamten monomeren und monomeren organischen Species berechnet (DIETZE 1990). Aus diesen Konzentrationen und dem $\mathrm{pH}$ Wert des Wassers läßt sich der Anteil der einzelnen monomeren anorganischen Verbindungen mit Hilfe eines Rechnerprogramms ermitteln (PRENZEL. 1986).

\subsection{Untersuchungen der Makrofauna}

Im Rahmen der biologischen Untersuchungen der vorliegenden Arbeit wurden die Makrozoobenthos-Gruppen Mollusca, Amphipoda, Ephemeroptera, Plecoptera und Coleoptera sowie die Emergenz der Ephemeropteren und Plecopteren bearbeitet. Darüber hinaus wurden fischereikundliche Untersuchungen durchgeführt. Die Gesamtzahl der bearbeiteten Benthosorganismen beträgt ca. 109.000 Individuen. Aus der Emergenz wurden ca. 32.000 Individuen erfaßt, ferner wurden 1.242 Fische gefangen.

\subsubsection{Beprobung und Bearbeitung des Makrozoobenthos}

An den Probestellen der Hauptuntersuchungsbäche (R1 - R3, S1 - S3) kam es zwischen April 1986 und Dezember 1988 zu insgesamt 23 Probenahmen. In den Nebenuntersuchungsbächen erfolgten zwischen April 1986 und Oktober 1987 insgesamt 8 Probenahmen (Tab. 2). Für die Beprobungen wurde ein modifizierter SURBER-Sampler (SURBER 1937) mit einer Maschenweite von $315 \mu \mathrm{m}$ und einer Sammelfläche von $900 \mathrm{~cm}^{2}$ verwendet (Abb. 4). An jeder Probestelle wurden drei Proben aus Bereichen genommen, die für den jeweiligen Gewässerabschnitt bezüglich Fließgeschwindigkeit und Choriotopstruktur repräsentativ waren. Hier- 
Tab. 2: Termine der Benthosprobenahmen

\begin{tabular}{|c|c|c|c|c|c|c|}
\hline & alle Probest. & & $\begin{array}{l}\text { Hauptunters. } \\
\text { bäche }\end{array}$ & $\begin{array}{c}\text { Nebenunters. } \\
\text { bäche }\end{array}$ & & $\begin{array}{l}\text { Hauptunters. } \\
\text { bäche }\end{array}$ \\
\hline 1986 & $\begin{array}{c}17 . / 24.04 \\
12 . / 19.06 \\
31.07 . / 06.08 \\
16 . / 23.10 \\
12 . / 18.12\end{array}$ & 1987 & $\begin{array}{l}31.03 \\
28.04 \\
02.06 \\
09.07 \\
04.08 \\
15.09 \\
14.10 \\
17.11 \\
15.12\end{array}$ & $\begin{array}{l}24.03 . / 07.04 \\
\text { 02.07./09.07 } \\
\text { 13.10./14.10. }\end{array}$ & 1988 & $\begin{array}{l}21.01 \\
16.03 . \\
19.04 \\
03.06 \\
13.07 \\
16.08 \\
26.09 . \\
01.11 \\
08.12\end{array}$ \\
\hline
\end{tabular}

bei wurde das Substrat innerhalb der von dem Klapprahmen des Sammlers abgegrenzten Fläche intensiv mit den Händen wiederholt aufgewirbelt, wobei Tiere und feineres Material von der Strömung ins Netz gespült wurden. Der gesamte Netzinhalt wurde anschließend in einen mit Wasser gefüllten 10 l-Eimer gegeben und durch ein $50 \mu \mathrm{m}$-Sieb geschüttet. Der Rückstand wurde in Kunststoffgefäße gegeben und mit Ethanol $(80 \%)$ fixiert und konserviert. Die Weiterbearbeitung der Tierproben im Labor umfaßte, getrennt für jede Teilprobe der Probenahmen, eine Vorsortierung durch Elutration (PAULY 1973) sowie die anschließende Aussortierung, Bestimmung und Zählung unter dem Stereomikroskop.

Für die Determination wurde die folgende Spezialliteratur verwendet:

- Mollusca: GLÖER et al. (1980)

- Crustacea: SCHELLENBERG (1942), SCHIMMER (1987b)

- Ephemeroptera: BUCK \& MERZ (1976), ELLIOTT et al. (1988), KIMMINS (1972), LANDA (1969), MÜLLER-LIEBENAU (1969), SCHOENEMUND (1930), SOWA (1970), THOMAS (1968)

- Plecoptera: AUBERT (1959), HYNES (1977), ILLIES (1955, 1963), KIS (1973)

- Coleoptera: BERTHELEMY (1962), FREUDE et al, (1971, 1979), HOLLAND (1972), KLAUSNITZER (1977), PANKOW (1979), STEFFAN (1958, 1961)

Die Systematik richtet sich bei den Coleopteren nach FREUDE et al. (1971, 1979), bei den restlichen Gruppen nach ILLIES (1978a). 


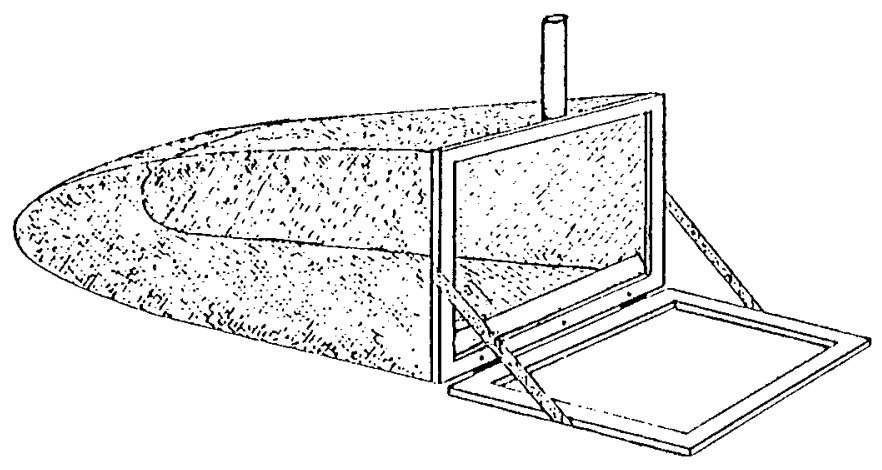

Abb. 4: Modifizierter SURBER-Sampler der Benthos-Untersuchungen

\subsubsection{Erfassung und Bearbeitung der Emergenz}

Die Ephemeropteren- und Plecopteren-Emergenz wurde durch dreibeinige, pyramidenförmige Emergenzfallen erfaßt, die mit feinmaschigem Gardinenstoff aus Kunstfasern bespannt sind (Abb. 5). Die Fallen sind unterhalb der Wasseroberfläche nach allen Seiten offen. Die überdeckte Wasseroberfläche beträgt zwischen 0,5 und $0,8 \mathrm{~m}^{2}$. An der Spitze befindet sich ein reusenförmiger Aufsatz, dessen Fanggefäß mit einem Alkohol/Glycerin-Gemisch (70:30) zur Fixierung und Konservierung der Tiere gefüllt wird. Die Aufstellung erfolgte so, daß ein für die Probestellen möglichst repräsentativer Choriotopbereich, an den größeren Gewässern im Randbereich, von den Fallen überdeckt wurde.

Die Emergenz wurde an den Probestellen der Hauptuntersuchungsbäche zwischen Ende Juni 1986 und März 1989 erfaßt. An den Nebenuntersuchungsbächen standen die Emergenzfallen zwischen Juli 1986 und Dezember 1987. 1986 kam es zu hochwasserbedingten, jeweils einwöchigen Ausfällen der Fallen Ende Juli an S2 und im September an S3. Von Anfang Dezember 1986 bis Ende März 1987 waren alle Fallen abgebaut, wohingegen die Fallen in den beiden darauffolgenden Wintern an den Hauptuntersuchungsbächen stehen gelassen wurden.

Die Leerung der Emergenzfallen erfolgte in den Sommerhalbjahren in der Regel in einwöchigen Abständen, zwischen Ende Oktober und Anfang März wurden 
Kontrollen im mehrwöchigen Rhythmus durchgeführt. Die gefangenen Tiere wurden den Fanggefäßen entnommen und in Ethanol (80\%) überführt. Die Determination erfolgte unter dem Stereomikroskop im Labor (Bestimmungsliteratur: s. Kap. 4.3.1).

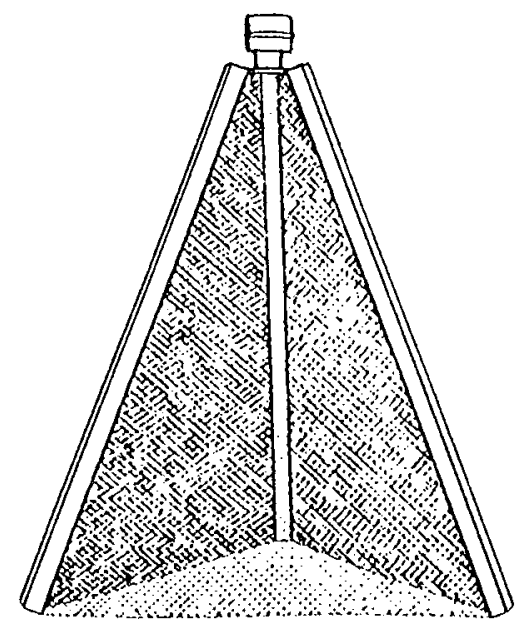

Abb. 5: Emergenzfalle

\subsection{Datenauswertung}

Abgesehen von der Wassertemperatur, bei der keine ausreichende Datenbasis gegeben ist, wurden aus allen gemessenen bzw. zur Verfügung gestellten physikalisch-chemischen Daten arithmetisches Mittel und Standardabweichung berechnet sowie Minimum- und Maximum-Wert zur Auswertung entnommen (MÜHLENBERG 1989).

Die Daten aus Benthosbeprobung und Emergenzerfassung wurden wie quantitative Daten ausgewertet, obwohl bei der angewandten Methodik nicht von einer quantitativen Erhebung ausgegangen werden kann (SCHWOERBEL 1986). Ziel der Untersuchungen war jedoch in erster Linie ein Vergleich der Struktur der benthischen Lebensgemeinschaften und nicht eine vollständige quantitative faunistische Erfassung, die eine wesentlich größere Zahl an Benthosproben 
(CHUTTER 1972) und größere Emergenzfallen (CASPERS 1980) erfordern würde. Unter diesem Vergleichsgesichtspunkt mit identischer methodischer Bearbeitung aller Probestellen ist eine quantitative Auswertung gerechtfertigt. In einer Reihe von Untersuchungen konnte nachgewiesen werden, daß bereits wenige, gezielt aus charakteristischen Bereichen entnommene Benthosproben ausreichen, um die Struktur der Biozönose zu erfassen. Nach CHUTTER \& NOBLE (1966) liefert die dreimalige Beprobung einer Fläche von jeweils ca. 0,1 $\mathrm{m}^{2}$ in Gewässern mit stärkerer Strömung ein repräsentatives Bild der Besiedlung. Bei anderen Untersuchungen wurden in den ersten beiden von zehn Beprobungen bereits $86 \%$ der Gesamtfauna gefunden (FROST et al. 1971).

Für die bei der Emergenzerfassung benutzten Fallen liegen eine Reihe positiver Erfahrungen vor IRAMACHERS \& BLANKE 1987, BLANKE 1988, WIPPERFÜRTH 1989, RÜDDENKLAU 1989, ROMMELMANN \& HEITKAMP 1989, WESTERMANN 1990). Bei den gewonnen Daten kann von einer für Vergleichszwecke ausreichenden quantitativen Erfassung ausgegangen werden. Einen wesentlichen Nachteil stellt beim Fang der Ephemeropteren der hohe Anteil an Subimagines dar, die bei vielen Gattungen nicht bis zur Art determinierbar sind.

Die Individuenzahlen aus den Teilproben der Benthosbeprobungen eines Termins wurden für jede Probestelle addiert und auf eine Fläche von $1 \mathrm{~m}^{2}$ umgerechnet. Diese Umrechnung auf eine Fläche von $1 \mathrm{~m}^{2}$ wurde auch für die Emergenzdaten vorgenommen. Die biologischen Daten wurden bezüglich der wichtigsten Parameter von Artengemeinschaften nach Artenzahl, Abundanz, Dominanzstruktur, Diversität und Evenness ausgewertet (MÜHLENBERG 1989). Bei den zu einem sehr hohen Anteil auf Artbasis vorliegenden Emergenz-Daten wurden darüber hinaus die biozönotische Ähnlichkeit der Probestellen miteinander verglichen und die Arten-Turnover-Rate für 1987/1988 an den Probestellen der Hauptuntersuchungsbäche berechnet.

Durch die Dominanz $\left(D_{i}\right)$ wird die relative Häufigkeit einer Art im Vergleich zu den übrigen Arten beschrieben:

$$
D_{i}=n_{i} / N
$$

$n_{i}:$ Individuenzahl Art $i$

$\mathrm{N}$ : Gesamtindividuenzahl 
Es wird nach SCHWERDTFEGER (1975) und PALISSA et al. (1979) unterschieden zwischen

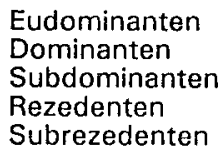

$$
\begin{aligned}
& >10 \% \\
& 5-10 \% \\
& 2-5 \% \\
& 1-2 \% \\
& <1 \%
\end{aligned}
$$

Zur Beschreibung der Diversität, dem Verhältnis der Verteilung der Individuen auf die Arten (PIELOU 1975), wurde der SHANNON-Index $\left(\mathrm{H}_{\mathrm{S}}\right)$ berechnet

$$
H_{S}=-\Sigma p_{i}^{*} \text { In } p_{i} \quad \text { mit } p_{i}=n_{i} / N
$$

$n_{i}:$ Anzahl Art i

$\mathrm{N}$ : Gesamtindividuenzahl

s : Gesamtartenzahl

Bei der Betrachtung des SHANNON-Index ist zu berücksichtigen, daß die Diversität der Biozönosen sowohl mit zunehmender Artenzahl als auch mit zunehmend gleichmäßiger Verteilung der Individuen auf die vorhandenen Arten steigt. Dem Diversitätsindex wird ein hoher Indikatorwert zugesprochen, da sich bei schädigenden Einflüssen in den Biozönosen sowohl ein Verlust an Arten als auch eine größere Ungleichheit in der Verteilung der Individuen auf die Arten bemerkbar macht (MÜHLENBERG 1989).

Die Evenness (E) beschreibt die Relation des Diversitätsindex zu dem theoretisch maximalen Indexwert bei gleicher Artenzahl

$$
E=H_{s} / H_{\max }=H_{s} / \ln s
$$

Für den biozönotischen Ähnlichkeitsvergleich der Probestellen anhand der Emergenzdaten wurde der WAINSTEIN-Index $\left(K_{W}\right)$ verwendet, der ein Produkt aus der Artenidentität nach JACCARD (JZ) und der Dominanzidentität der RENKONEN-Zahl $\left(R_{e}\right)$ ist. Dazu sind folgende Berechnungen notwendig

$$
\begin{gathered}
J Z=G /\left(S_{A}+S_{B}-G\right) \\
R_{e}=\Sigma \min D_{A, B} \\
K_{W}=R_{e} * J Z
\end{gathered}
$$


$S_{A, B}$ : Artenzahl an Stelle A bzw. B

$G$ : gemeinsame Artenzahl der beiden Probestellen

min $D_{A, B}$ : der kleinere Dominanzwert der gemeinsamen Arten von $A$ und $B$

Die Arten-Turnover-Rate (T) ergibt sich aus

$$
T=(J+E) /\left(S_{1}+S_{2}\right)
$$

J: Anzahl der neuen Arten

E: Anzahl der verschwundenen Arten

$\mathrm{S}_{1}$ : Anzahl der Arten im Jahr 1

$S_{2}$ : Anzahl der Arten im Jahr 2

In Ergänzung zu diesen Analysen wurde der Artenfehlbetrag (AF) nach KOTHE (1962) für die einzelnen Probestellen berechnet

$$
A F=\left(A_{1}-A_{X}\right) * 100 / A_{1}
$$

$A_{1}:$ Artenzahl an der Referenzstelle

$A_{X}$ : Artenzahl der durch Schadstoffe belasteten Stelle

Alle Korrelationsanalysen wurden mit dem Computer-Statistikprogramm SPSS/PC + durchgeführt, das eine PEARSONsche Produkt-Moment-Korrelationsmatrix ersteilt (UEHLINGER 1988). Die Daten wurden vorher auf Normalverteilung geprüft.

Eine Analyse der Ähnlichkeitsstrukturen unter den Probestellen bezüglich einzelner oder einer Gruppe von Faktoren erfolgte anhand von Clusteranalysen, die numerische Klassifikationsverfahren darstellen, die in der Lage sind, in einer Grundgesamtheit an Objekten homogene Untermengen, sogenannte Cluster, zu entdecken. Die Ergebnisse lassen sich in Dendrogrammform darstellen.

Das Verfahren der Clusteranalyse wurde benutzt, um Gruppen weitestgehend gleicher abiotischer Bedingungen im Hinblick auf die Choriotopstrukturen, des zeitlichen Gangs der pH-Werte und der hydrochemischen Parameter zwischen den Probestellen herauszustellen. Die Ergebnisse des biozönotischen Ähnlichkeitsvergleichs der Emergenzerfassung wurden ebenfalls einer Clusteranalyse unterzogen und bieten so die Möglichkeit zu einem Vergleich mit den Ergebnissen der Analysen der abiotischen Faktoren, um deren Einfluß auf die Ausbildung der Biozönosen abschätzen zu können. 
Die abiotischen Daten wurden jeweils unter Verwendung der quadrierten euklidischen Distanz als Proximitätsmaß und den Fusionierungsalgorithmen des SingleLinkage-Verfahrens und des Verfahrens nach WARD mit Hilfe des ComputerProgramms SPSS/PC + analysiert (SCHUBÖ \& UEHLINGER 1986, UEHLINGER 1988). Das erstgenannte Verfahren ist insbesondere geeignet, stark abweichende Fälle ("Ausreißer") zu erkennen. Gruppen mit relativ geringen Unterschieden können hingegen nicht ermittelt werden. Das Verfahren nach WARD stellt in der Regel das zur Gruppenbildung am besten geeignete Verfahren dar (BACKHAUS et al. 1989).

Da die Abundanzdaten der Emergenzerfassung nicht die Voraussetzungen zur unmittelbaren Durchführung einer Clusteranalyse erfüllen, wurden die Ergebnisse der Berechnung der WAINSTEIN-Indices dem von MOUNTFORD (1962) beschriebenen monothetischen Analyseverfahren unterzogen.

\subsection{Untersuchungen der Fischfauna}

Zur Erfassung der Fischfauna wurden an jeder Probestelle der Hauptuntersuchungsbäche fünf Elektrobefischungen (HALSBAND \& HALSBAND 1975. LAGLER 1978) mit dem Gerät IG 80 der Fa. Grassl durchgeführt. Das IG 80 ist ein tragbares, batteriebetriebenes Gleichstrom-Impulsgerät. Es wurde eine Seilkathode benutzt.

An den Probestellen der Hauptuntersuchungsbäche erfolgten die Befischungen im Oktober 1986, Mai 1987, Dezember 1987, Mai 1988 und Oktober 1988. An den Probestellen der Nebenuntersuchungsbäche wurden jeweils drei Befischungen an den ersten drei der angebenen Termine vorgenommen. Bei jeder Befischung wurde eine etwa $100 \mathrm{~m}$ lange Gewässerstrecke nach Absperrung durch Netze bachaufwärts im Wasser gehend einmal abgefischt. Die gefangen Tiere wurden entnommen, auf äußerlich sichtbare Krankheitsbilder oder Mißbildungen untersucht, die Art bestimmt, die Totallänge auf $0,5 \mathrm{~cm}$ genau gemessen und das Gewicht der über $4 \mathrm{~g}$ schweren Fische mittels einer elektronischen Waage auf $2 \mathrm{~g}$ genau bestimmt. Alle Tiere wurden wieder ins Gewässer zurückgesetzt.

Vor Beginn der fischereikundlichen Untersuchungen wurde nach der sukzessiven Leerfangmethode (RALEIGH \& SHORT 1981) untersucht, wieviele Befischungs- 
durchgänge zur Erzielung einer quantitativen Abfischung in den zu untersuchenden Gewässern notwendig sind. Dabei wurde festgestellt, daß in diesen relativ schmalen und auch bei mittleren Wasserständen recht flachen Bächen bei der ersten Abfischung bereits $80-90 \%$ der über $5 \mathrm{~cm}$ großen Tiere gefangen werden. $100 \%$ werden in der Regel nach der zweiten Befischung erreicht. Kleinere Fische können hingegen mit der angewandten Methode nicht annähernd quantitativ erfaßt werden. Selbst bei bis zu drei Befischungen ergibt sich noch eine relativ hohe Fehlerquote. Unter diesen Gesichtspunkten wurde eine einmalige sorgfältige Abfischung als ausreichend betrachtet.

Der Auswertung wurden die tatsächlich gefangenen und gesichteten Individuenzahlen zugrunde gelegt. Die Anzahl der gefangenen Tiere wurde traditionsgemäß auf eine Wasserfläche von 1 ha umgerechnet. Zur Beurteilung des Ernährungszustands der Fische wurde aus Gewicht (g) und Länge (TL) der Tiere der Korpulenzfaktor (K) errechnet (JENS 1981):

$$
K=(g * 100) / T L^{3}
$$

Für die Abgrenzung der Altersklassen wird das Kalenderjahr zur Einteilung verwendet (LELEK 1961 zit. nach BRUNKEN 1988). Zur Altersklasse $0+$ gehören die Tiere, die im Frühjahr schlüpfen, bis zum Ende desselben Jahres. Mit Beginn des neuen Jahres treten sie in die Altersklasse $1+$ ein. 


\section{Ergebnisse}

5.1 Die Probestellen und ihre abiotischen Charakteristika

5.1.1 Geographie, Geologie, Pedologie

Die für die Untersuchungen ausgewählten 15 Probestellen liegen alle im Epirhithral der sechs untersuchten Fließgewässersysteme (Abb. 6). Den örtlichen Schwerpunkt der Untersuchungen bildete die im Südwestharz gelegene Sösemulde mit den Bächen Große Söse und Alte Riefensbeek (Abb. 7). Hinzu kamen die ebenfalls in der Sösemulde liegenden Bäche Großer Mollenbach/Große Schacht sowie nördlich von Braunlage Große Bode/Warme Bode und im nördlichen bzw. nordwestlichen Harzbereich Lange Bramke und Varleybach (Abb. 811).

\subsubsection{Das Hauptuntersuchungsgebiet Sösemulde mit Großer Söse und Alter Riefensbeek}

Die Sösemulde wird im Nordwesten durch den nur schwach über die sich anschließende Oberharzer Hochfläche hinausragenden Oberharzer Diabaszug, der die Wasserscheide zum Einzugsgebiet der Innerste bildet, mit Höhen von etwa $600 \mathrm{~m}$ begrenzt. Im Südosten bildet der Höhenzug des Ackers mit Höhen um $800 \mathrm{~m}$ die Grenze zum Einzugsgebiet der Sieber. Zum Oker-Gebiet, das sich im Nordosten anschließt, verläuft die Wasserscheide teilweise entlang des künstlich angelegten Dammgrabens. Die Sösetalsperre (Baujahr 1931, 1,21 km², 25 Mio $\mathrm{m}^{3}$ ) schließt das Tal zum Harzrand hin ab. Die Muldenachse wird von Kleiner Söse und Söse gebildet, wobei die Lauflänge zwischen der Quelle der Kleinen Söse und dem Pegel Marienthal unterhalb der Talsperre $12,5 \mathrm{~km}$ beträgt. Bei einer Einzugsgebietsgröße von $50,0 \mathrm{~km}^{2}$ errechnet sich eine mittlere Talbreite von $4 \mathrm{~km}$. Die Hänge sind häufig sehr steil, und das Gebiet weist dadurch insbesondere auf der Ackerseite eine große Reliefenergie auf. Das Gewässernetz ist relativ gleichmäßig über das Einzugsgebiet verteilt. Die Bäche an den Flanken der Mulde verlaufen quer zum Schichtstreichen und sind zum großen Teil an Verwerfungszonen gebunden (SIEBERT 1988). Die Söse entwässert über Rhume, Leine, Aller und Weser in die Nordsee.

Die ältesten Gesteine sind untermitteldevonische Tonschiefer. Der Oberharzer Diabaszug wird vor allem aus mitteldevonischen und unterkarbonischen Tonschiefern, Kieselschiefern, Wechsellagerungen aus Kalkstein, Tonschiefern 


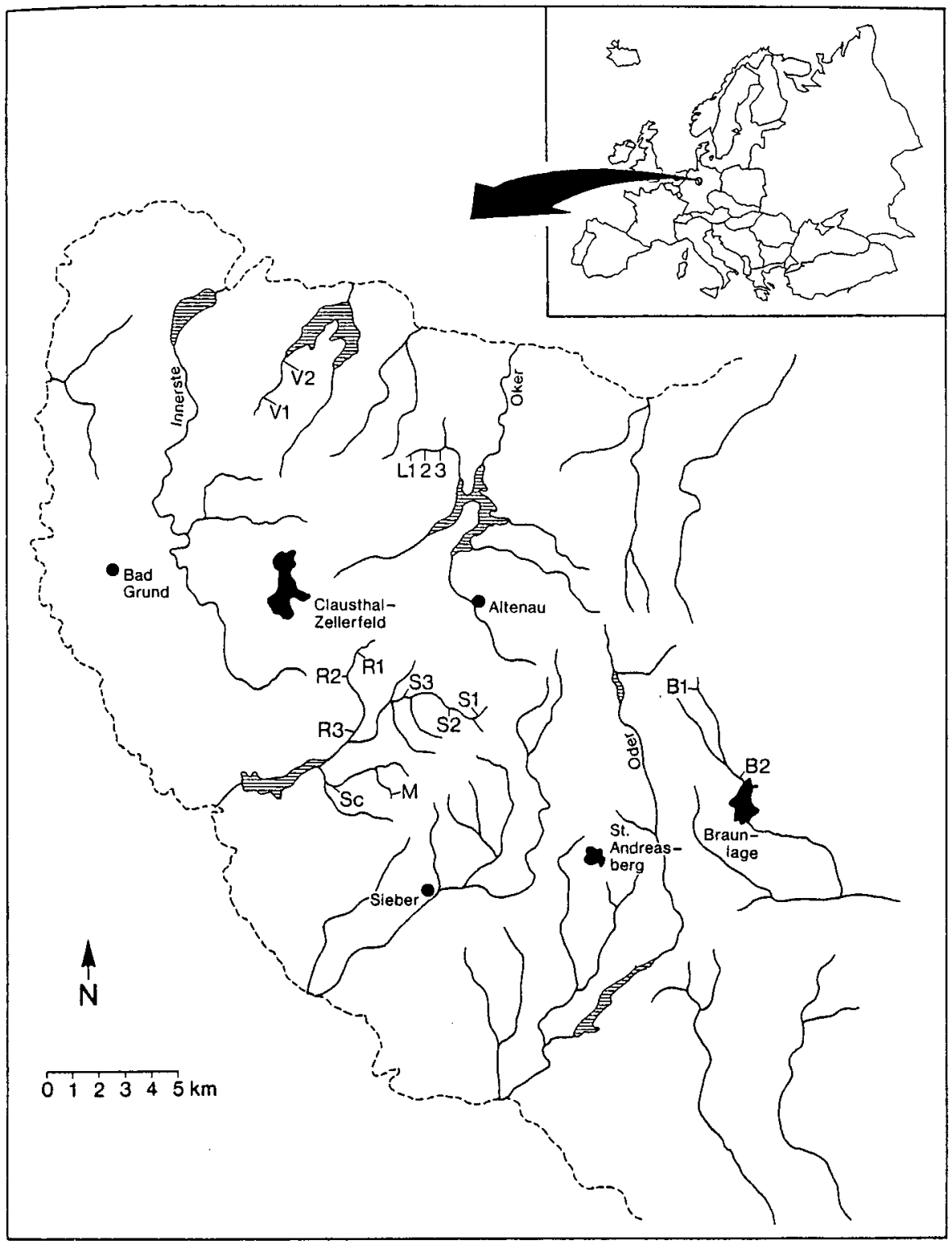

Abb. 6: Übersichtskarte des westlichen Harzteils mit den Probestellen 


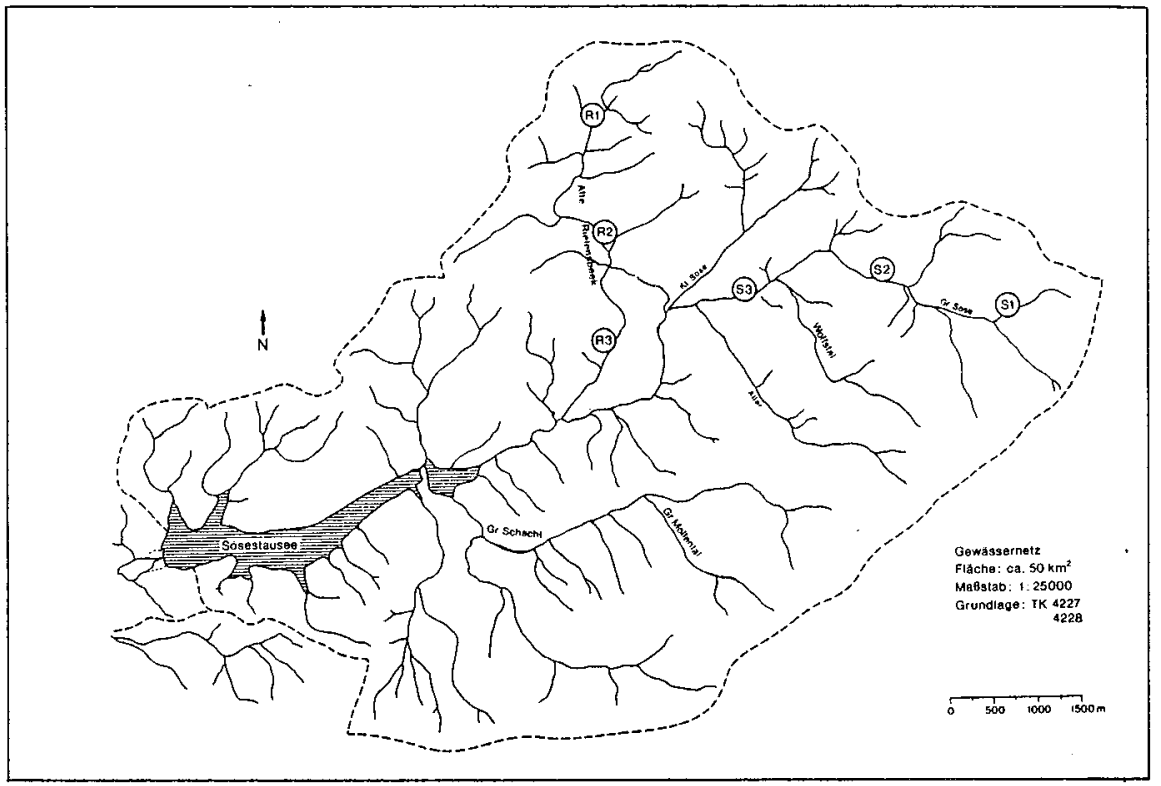

Abb. 7: Die Hauptuntersuchungsstellen im Ėinzugsgebiet des Sösestausees

und Grauwacken sowie Spiliten und Tuffen gebildet. Der Acker-Bruchberg-Zug besteht dagegen aus verwitterungsbeständigen Quarziten des Oberdevon bis Unterkarbon (Acker-Bruchberg-Quarzit), oberdevonischen Tonschiefern, unterkarbonischen Plattenschiefern, Grauwacken und Kieselschiefern. Die Sösemulde selbst weist vorwiegend Tonschiefer und Grauwacken des Karbon sowie KulmKieselschiefer, Tonschiefer, Grauwacken, Konglomerate und oberdevonische Tonschiefer auf (ROOSTAI 1987, SIEWERS \& ROOSTAI 1990, MOHR 1984). In Bezug auf die Pufferungskapazität von Säureeinträgen ergibt sich eine Einstufung von empfindlich (Quarzit) über intermediär (Tonschiefer, Grauwacke) bis unempfindlich (Diabas), wobei die einzelnen Bereiche gut voneinander getrennt sind (MALESSA \& ANDREAE 1990).

Die Böden des Sösetals sind relativ einheitlich gebaut. An den steilen Hängen wirkt die Erosion der Bildung gewachsener mächtiger Böden entgegen. Das Bild 

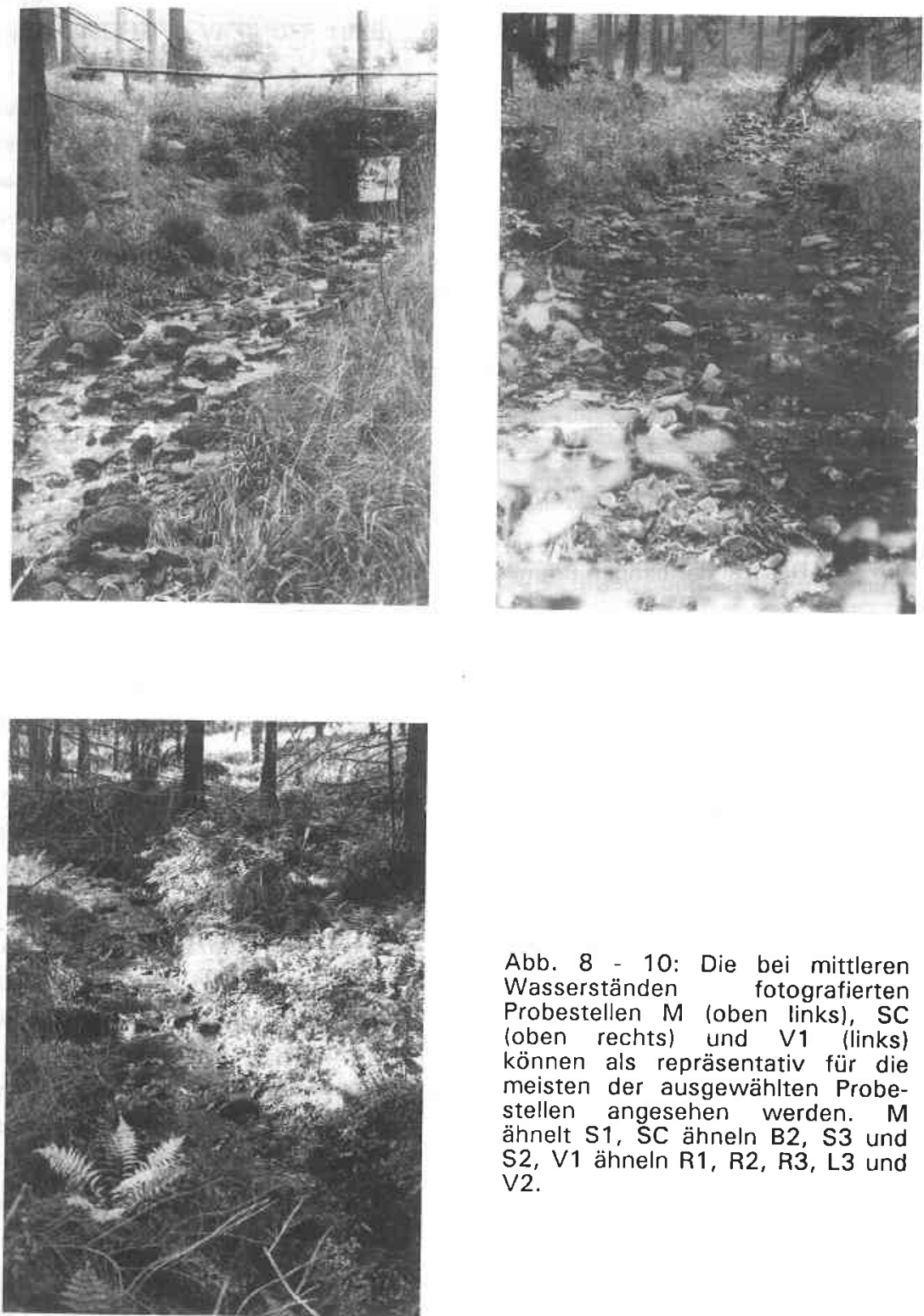

Abb. 8 - 10: Die bei mittleren Wasserständen fotografierten Probestellen $M$ (oben links), SC (oben rechts) und V1 (links) können als repräsentativ für die meisten der ausgewählten Probestellen angesehen werden. $M$ ähnelt S1, SC ähneln B2, S3 und S2, V1 ähneln R1, R2, R3, L3 und $\mathrm{V} 2$. 


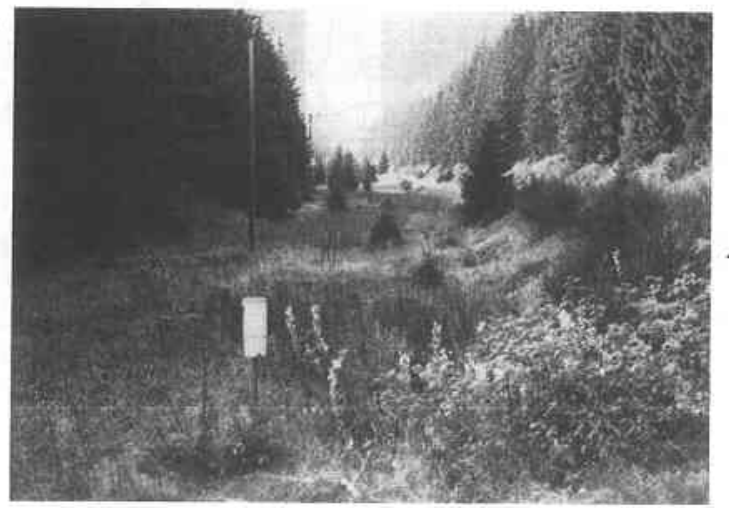

Abb. 11: Das Tal der Langen Bramke zwischen den Probestellen L1 und L2

wird von flachen bis mittleren Braunerden geprägt. Auf dem Oberharzer Diabaszug und den angrenzenden Solifluktionsdecken zählen diese Braunerden zu den basenreichsten Böden im Harz. Im Bereich der Wechsellagerungen von Tonschiefer und Grauwacke sind schluffige, lehmige, teilweise tiefgründige, podsolierte oder podsolige Braunerden mit mittlerer Basensättigung zu finden. Auf dem Acker-Bruchberg-Quarzit haben sich Podsol-Pseudogleye, Stagnogleye und Hanggleye oft mit Anmoorcharakter gebildet. Der Kammbereich wird außerdem durch bis zu $6 \mathrm{~m}$ mächtige Moorauflagen geprägt, die teilweise ein Wasserreservoir für Quellen am Hang des Ackers darstellen (SCHWIETERT 1989 zit. nach MALESSA \& ANDREAE 19901.

Ein zusammenhängender Grundwasserstrom wird durch die zahlreichen Schichtgrenzen, Schuppenbahnen, Störungen und Tonschieferlagen verhindert. Dies führt zu einer relativ hohen Quellendichte von mehr als 2 Quellen $/ \mathrm{km}^{2}$, was einem oberirdischen Einzugsgebiet von $0,10-0,13 \mathrm{~km}^{2}$ bei sehr geringen Schüttungsmengen entspricht. Die meisten der Quellen sind intermittierende Hangschuttquellen, die in Trockenzeiten versiegen. Der größte Teil des Abflusses, auch der von Hochwasserwellen, besteht aus kurzfristig gebildetem Sicker- und Grundwasser, das wegen der geringen Dichte der gut wasserdurchlässigen Verwitterungsschichten und der starken Hangneigungen nicht gespeichert 
werden kann und schon nach kurzer Zeit wieder an die Oberfläche tritt (SIEBERT 1988, SIEBERT \& VIERHUFF 1989).

Bei hohen Niederschlägen kommt es zudem in den Bereichen geringer Bodenbedeckung zu einem erheblichen oberflächlichen Abfluß in die Gewässer, der verbunden ist mit einem beträchtlichen Abtrag von Gesteins- und Bodenmaterial. Aưfgrund der überquadratischen Flächengestalt muß dabei das Sösetal oberhalb der Ortschaft Riefensbeek als sehr hochwasserempfindlich angesehen werden.

Große Söse (S1 - S3)

Die Quelle der Großen Söse befindet sich in $790 \mathrm{~m}$ Höhe, liegt im Quarzit und hat ein Einzugsgebiet aus Quarzit, Kieselschiefern und Hochmoortorf von ca. $0,04 \mathrm{~km}^{2}$. Sie ist umgeben von einzelnen hohen und mittelhohen Fichten. Der schwammige Boden ist dicht mit Gräsern bewachsen. Die Entwässerung erfolgt großräumig über zahlreiche kleine Rinnsale, die sich nach und nach zum eigentlichen Bach vereinigen.

Das gesamte Einzugsgebiet der Großen Söse, das zu 60 \% aus Quarzit, $20 \%$ aus Grauwacken/Tonschiefer-Wechsellagerungen, $15 \%$ aus Schiefer-Serien und zu $5 \%$ aus Diabas besteht, umfaßt eine Fläche von $11,6 \mathrm{~km}^{2}$ (SIEBERT 1988). Es erreicht eine Höhe von maximal $865 \mathrm{~m}$. Im östlichen oberen Teil ist ein steiler Geländeabfall nach Nordwesten zu verzeichnen, so daß Westhänge vorherrschen.

Bis zum Zusammenfluß mit der Kleinen Söse oberhalb von Kamschlacken bei Flußkilometer 5,1 hat der Bach rechtsseitige Zuflüsse nur im Kleinen und Großen Ifental. Von links kommen Bäche aus dem Großen und Kleinen Morgenbrodstal, Wolfstal und Allertal. Bei km 1,0 schneidet der Morgenbrodstaler Graben die Quelläste der Großen Söse mit einer Einzugsgebietsfläche von $3,5 \mathrm{~km}^{2}$ in $600 \mathrm{~m}$ Höhe ab. Durch den Graben werden maximal $0,36 \mathrm{~m}^{3}$ in das Innerste-Einzugsgebiet abgeführt, was der 3,3-fachen, langjährigen mittleren Wassermenge an der Ableitungsstelle entspricht, die im Mittel an 350 Tagen im Jahr nicht überschritten wird (HAASE et al. 1970). Da sich die Absperrung jedoch in einem schlechten baulichen Zustand befindet, ist gewährleistet, daß die sich unterhalb anschließende Gewässerstrecke zu keinem Zeitpunkt des Jahres trocken fällt. 
Alte Riefensbeek (R1 - R3)

Der Großen Söse nordwestlich gegenüber, an den Hängen des Oberharzer Diabaszuges entspringt in einem hohen Fichtenforst auf tiefgründiger Braunerde in $590 \mathrm{~m}$ Höhe die Alte Riefensbeek. Im etwa 0,04 km² großen Quelleinzugsgebiet sind neben Diabas Grauwacken/Tonschiefer-Wechsellagerungen zu finden. Der Bach hat nur wenige kleinere Zuflüsse. Das gesamte Einzugsgebiet der Alten Riefensbeek mit Höhen von maximal $620 \mathrm{~m}$ hat eine Fläche von $6,0 \mathrm{~km}^{2}$. Es zeigt insgesamt einen Abfall nach Südsüdost mit Hangexpositionen in alle Himmelsrichtungen. Der Untergrund besteht zu $75 \%$ aus Grauwacken/Tonschiefer-Wechsellagerungen, $15 \%$ aus Kieselschiefer und 10 $\%$ aus Diabas. Im Einzugsgebiet der Probestelle R1 hat der Diabas einen Anteil von $65 \%$, bis R2 sind es noch $14 \%$ bei steigenden Anteilen an Grauwacke und Kieselschiefer (SIEBERT 1988, HWW 1990).

\subsubsection{Großer Mollenbach (M) und Große Schacht (SC)}

Das Einzugsgebiet der Großen Schacht mit dem Großen Mollenbach als einem der Hauptzuflüsse schließt sich südwestlich auf der Ackerseite an das Einzugsgebiet der Großen Söse an. Das Quellgebiet des Großen Mollenbachs liegt in $770 \mathrm{~m}$ Höhe auf Quarzit. Die Hauptfließrichtung ist der Hangneigung folgend nach Nordwesten gerichtet.

Die Große Schacht bildet ein parallel zum Sösetal in südwestlicher Richtung verlaufendes Tal. Das $6,1 \mathrm{~km}^{2}$ große Einzugsgebiet besteht zu $50 \%$ aus Grauwacke und Tonschiefern, $30 \%$ Quarzit und $20 \%$ Diabas. Bis Probestelle M hat der Quarzit einen Anteil von $82 \%$, Tonschiefer machen $18 \%$ aus (HWW 1990).

\subsubsection{Große Bode (B1) und Warme Bode (B2)}

Die Große Bode entspringt auf einer Hochfläche am Südsüdwesthang des Brockens im Bereich des Bodebruch-Moorgebietes. Durch den Zusammenfluß von Großer Bode und Kleiner Bode entsteht nordnordwestlich von Braunlage die Warme Bode, die über Saale und Elbe in die Nordsee entwässert. Der Untergrund des Einzugsgebiets besteht aus Brockengranit. Große Teile der Hochfläche 
im Queligebiet der Großen Bode sind vermoort, auf den restlichen Flächen sind Podsole und Podsol-Ranker zu finden (GROTH \& TRAPPHOFF 1988).

\subsubsection{Lange Bramke (L1 - L3)}

Die Lange Bramke gehört über die Große Bramke zu den Zuflüssen des Okerstausees. Östlich der Schalke gelegen fließt sie nach Ostnordost. Das Einzugsgebiet besteht aus unterdevonischem Kahlebergsandstein, wobei die Schalker, Giengelsberger und Nessigi-Schichten an die Oberfläche treten, die sich aus dickbankigen Quarziten, feinkörnigem quarzitischem Sandstein und sandig-siltigen Schiefern und Tonschiefern zusammensetzen. Im Bereich der Probestelle L3 sind außerdem Wechsellagerungen von Tonschiefern und Kalksandsteinbänken (Speciosus-Schichten) und karbonatreiche Tonschiefer zu finden. Hänge und Hangfußzonen sind mit skelettreichen Solifluktionserden bedeckt (HINZE 1971). Die Böden im Einzugsgebiet sind überwiegend Podsol-Braunerden sowie Podsole im Kammbereich, die teilweise in Bachnähe hydromorph überprägt sind (GEENEN 1976). Im oberen Talbereich ist ein Hangmoor ausgebildet.

\subsubsection{Varleybach (V1 - V2)}

Der Varleybach ist einer der drei Hauptzuflüsse des Granestausees. Die Hauptfließrichtung ist Nordnordost. Soweit das Wasser des Stausees nicht für die Trinkwassergewinnung entnommen wird, fließt es über Innerste, Leine, Aller und Weser der Nordsee zu. An Gesteinen finden sich im Einzugsgebiet dunkle Tonschiefer, graue Tonschiefer mit Kalkknollen und Kalkknollenschiefer sowie Lydite und Grauwacken. Die Pedologie wird vor allem von Braunerden bestimmt, die relativ hohe Calcium-Gehalte aber wenig Magnesium, Natrium und Kalium enthalten (HWW 1990).

\subsubsection{Wetter und Schadstoffdeposition im Untersuchungszeitraum}

\subsubsection{Wetter}

Von den acht über den Harz verteilten Meßstationen des Deutschen Wetterdienstes befinden sich die fünf Stationen Clausthal (Abb. 12), Altenau, Braunlage, Schulenberg und Hahnenklee relativ dicht an den Probestellen. Die 


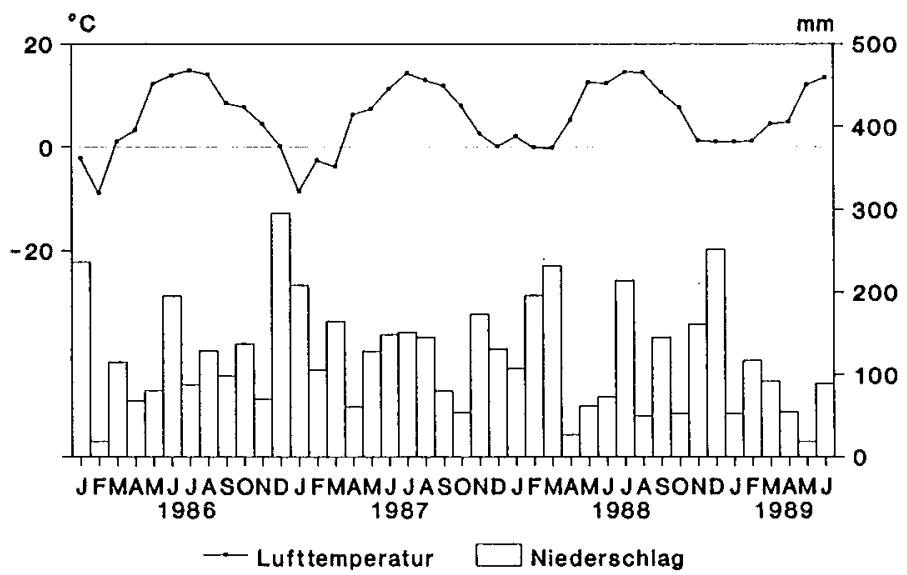

Abb. 12: Monatliche Mittel der Lufttemperatur und monatliche Niederschlagssummen an der Meßstation Clausthal

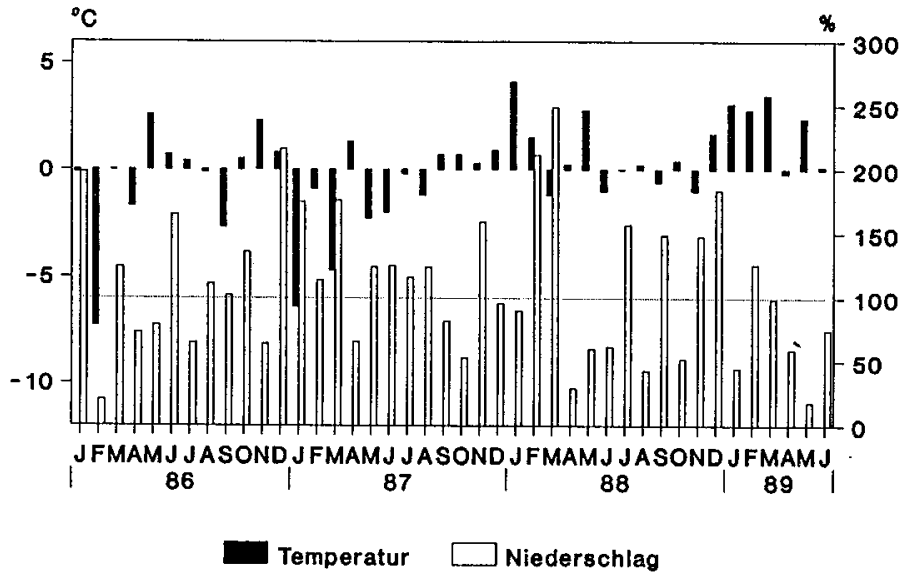

Abb. 13: Monatsmitteltemperaturen und monatliche Niederschlagssumme der Meßstation Clausthal im Vergleich mit den langjährigen Mittelwerten 1951-1980 
Daten aller fünf Stationen zeigen für die Jahre 1986 - 1989 einen identischen Verlauf der monatlichen Temperaturmittel (DWD 1986-1989). Die jeweiligen Abweichungen um einige zehntel Grad erklären sich aus den unterschiedlichen Höhenlagen. Für die Wetterstation Clausthal ergeben sich im Vergleich zum langjährigen Mittel 1951-1980 folgende Abweichungen (Abb. 13): Temperatur $1986-0,4{ }^{\circ} \mathrm{C}, 1987-1,2{ }^{\circ} \mathrm{C}, 1988+0,6{ }^{\circ} \mathrm{C}$; Niederschlag $1986+223$ $\mathrm{mm}, 1987+239 \mathrm{~mm}, 1988+261 \mathrm{~mm}$.

1986 zeichnete sich insbesondere durch einen kalten Winter mit sehr niedrigen Temperaturen im Februar aus. Bereits sommerlichen Temperaturen im Mai folgte ein ebenfalls warmer Sommer. Der September war deutlich kühler als im langjährigen Mittel. Auch die ersten drei Monate des Jahres 1987 brachten sehr niedrige Temperaturen. Nach einem kühlen Sommer lagen die Temperaturen erst im Herbst wieder im Bereich des langjährigen Mittels. 1988 folgten ein sehr milder Winter und ein mildes Frühjahr, dem sich ein durchschnittlicher Sommer und Herbst anschlossen. 1988/89 gab es einen weiteren sehr milden Winter.

In beiden warmen Wintern konnte sich nur zeitweilig eine dünne Schneedecke ausbilden, die wiederholt abtaute. Die Winter 1985/86 und 1986/87 brachten hingegen hohe Schneedecken, die bis in den April liegen blieben. Tauwetter und hohe Niederschläge führten an der Jahreswende 1986/87 zu extremen Hochwässern (vgl. Kap. 5.1.3.1).

Die Niederschläge verteilen sich sehr ungleichmäßig und unregelmäßig über den Untersuchungszeitraum (Abb. 12). Allgemein wiesen alle Winterhalbjahre hohe Niederschlagsmengen auf, die deutlich über den im langjährigen Mittel gemessenen Werten lagen. Auch der Sommer 1987 war insgesamt zu feucht. Die hohe Niederschlagssumme für den Juni 1986 erklärt sich aus wenigen sehr hohen Tagesniederschlägen in der ersten Juniwoche, die am 07.06. zu einer Hochwasserwelle führten.

Für die Sösemulde wurde für den Zeitraum 1941-1965 eine mittlere jährliche Niederschlagsmenge von $1310 \mathrm{~mm}$ errechnet, von denen 50,6\% im Winterhalbjahr und $49,4 \%$ im Sommerhalbjahr gefallen sind (HAASE et al. 1970). SIEBERT (1988) konnte durch seine Untersuchungen zeigen, daß die Niederschlagsmengen an den nach Nordwesten exponierten Hängen des Ackers in der Zeit von April bis November 1987 nicht höher waren als die Niederschlagsmengen in der nördlichen Hälfte der nach Südosten abfallenden Hänge des Oberhar- 
zer Diabaszuges. Sowohl für das Einzugsgebiet von Großer Söse als auch Alter Riefensbeek wurden in den acht Monaten $1020 \mathrm{~mm} / \mathrm{m}^{2}$ gemessen.

\subsubsection{Atmosphärische Schadstoffdeposition}

Über die Atmosphäre werden erhebliche Schadstoffmengen in die Waldökosysteme eingetragen. Hauptursache für den Sauren Regen ist die Störung der hydrogeochemischen Kreisläufe insbesondere durch die Verbrennung fossiler Brennstoffe mit ihren hohen Schwefeldioxid- und Stickoxidemissionen. Schwefeldioxid $\left(\mathrm{SO}_{2}\right)$ wird in der Atmosphäre über schwefelige Säure $\left(\mathrm{H}_{2} \mathrm{SO}_{3}\right) \mathrm{zu}$ Schwefelsäure $\left(\mathrm{H}_{2} \mathrm{SO}_{4}\right)$ unter photochemischer Stimulation oxidiert. Ähnlich verläuft die Oxidation der Stickoxide zu Salpetersäure $\left(\mathrm{HNO}_{3}\right)$. Bei der Deposition von Ammonium werden ebenfalls zur Versauerung beitragende Protonen freigesetzt. In geringem Umfang können außerdem Aluminium-, Eisen- und Mangan-Depositionen als Kationensäuren einen Anteil an der Versauerung haben (STUMM et al. 1983, OVERREIN 1983).

Aufgrund der unterschiedlichen Dauer der Umwandlungsprozesse unterliegt Schwefeldioxid am stärksten einem Ferntransport, der bei den Stickoxiden eine wesentlich geringere Rolle spielt. Ammoniak wird ausschließlich in der Nähe des

Tab. 3: Ergebnisse der Niederschlagsuntersuchungen im Einzugsgebiet von Großer Söse und Alter Riefensbeek (nach SIEWERS \& ROOSTAI 1990)

\begin{tabular}{lccr} 
& $\mathrm{pH}$ & $\begin{array}{r}\mathrm{SO}_{4} \\
{[\mathrm{mg} / \mathrm{l}}\end{array}$ & \multicolumn{1}{c}{$\begin{array}{c}\mathrm{NO}_{3} \\
{[\mathrm{mg} / \mathrm{l}]}\end{array}$} \\
\hline Median & 3,8 & 12,1 & 8,4 \\
Min & 2,8 & 2,3 & 1,3 \\
Max & 7,6 & 56,0 & 59,1 \\
\hline
\end{tabular}

Tab. 4: Durchschnittliche an Meßstationen im Harz gemessene Depositionsraten im Untersuchungszeitraum in $\mathrm{g} / \mathrm{m}^{2 *}$ a (nach ZAHN 1990)

\begin{tabular}{|c|c|c|c|c|c|}
\hline & $\begin{array}{l}\text { Riefensbeek } \\
\text { Freiland }\end{array}$ & Bestand & $\begin{array}{l}\text { Hohestein } \\
\text { Freiland }\end{array}$ & Bestand & $\begin{array}{l}\text { Lange Bramke } \\
\text { Bestand }\end{array}$ \\
\hline $\mathrm{SO}_{4}$ & 6,90 & 69,92 & 7,57 & 37,70 & 20,18 \\
\hline $\mathrm{NO}_{3}-\mathrm{N}$ & 0,93 & 7,75 & 0,88 & 3,90 & 1,08 \\
\hline $\mathrm{NH}_{4}-\mathrm{N}$ & 0,85 & 4,00 & 0,89 & 2,83 & 0,91 \\
\hline
\end{tabular}


Emittenten deponiert. Die Deposition im Freiland erfolgt hauptsächlich als nasse Deposition mit Regen und Schnee. Dagegen ist der Eintrag unter Baumbestand durch den hohen Anteil trockener Deposition in Europa stark erhöht. Die höchsten Werte treten unter Fichtenaltbeständen auf. Neben der Art des Bestandes kommt der Exposition im Hinblick auf die Auskämmwirkung des Waldes eine große Bedeutung zu. Luvseitige Hänge und Waldränder mit hohem Luftmassendurchsatz und hoher Nebelhäufigkeit wie die westexponierten Hänge des Ackers in der Sösemulde weisen die höchsten Schadstoffeinträge auf (LINKERSDÖRFER \& BENECKE 1987, SCHOEN 1989).

Für das Harzgebiet stehen Daten über den Schadstoffeintrag für die drei Standorte Riefensbeek, Lange Bramke und Hohestein (westlich der Innerstetalsperre, in der Nähe des Varleybacheinzugsgebiets) durch die Meßprogramme des Niedersächsischen Landesamtes für Wasser und Abfall (LAWA 1988), Messungen im Rahmen der Falistudie Harz in der Sösemulde und Messungen des Instituts für Bodenkunde und Waldernährung der Universität Göttingen im Einzugsgebiet der Langen Bramke zur Verfügung. Der pH des Bestandesniederschlags hatte sowohl am Hohenstein als auch bei Riefensbeek einen mittleren Wert von 3,6, was gut mit dem von SIEWERS \& ROOSTAI (1990) ermittelten Median von 3,8 übereinstimmt (Tab. 3). Der niedrigste Wert lag in Riefensbeek bei pH 1,9.

Die bei Riefensbeek registrierte Sulfatdeposition im Bestand von im Mittel des Untersuchungszeitraums $69,92 \mathrm{~g} / \mathrm{m}^{2 *}$ a stellt eine Höchstbelastung in den alten Bundesländern dar (Tab. 4). Auch der nordwestliche Harz ist hoch belastet, wohingegen man an der Langen Bramke bei einer Sulfat-Deposition von 20,18 $\mathrm{g} / \mathrm{m}^{2 *}$ a von einer mittleren Belastung sprechen kann. Auch bei der Gesamtstickstoff-Deposition wurden an der Meßstation bei Riefensbeek mit 11,75 $\mathbf{g} / \mathrm{m}^{2 *}$ a Höchstwerte unter Bestand gemessen. An der Langen Bramke ergaben sich dagegen nur $1,99 \mathrm{~g} / \mathrm{m}^{2 *}$ a. Aus den Meßwerten errechnet sich eine Säurebelastung an der Station Riefensbeek von 18,27 kmol IÄ $\mathrm{H}^{+} / \mathrm{ha}{ }^{*} \mathrm{a}$, am Hohenstein von $10,13 \mathrm{kmol} I \ddot{A} \mathrm{H}^{+} / \mathrm{ha}^{*}$ a und an der Langen Bramke von $4,27 \mathrm{kmol} I \ddot{A}$ $\mathrm{H}^{+}$/ha*a (ZAHN 1990).

Darüber hinaus werden weitere Elemente in erheblichem Umfang in die Ökosysteme eingetragen. Im Einzugsgebiet der Sösetalsperre mit einer Fläche von 50 $\mathrm{km}^{2}$ ist u.a. mit einer jährlichen Deposition von 3,5 t Zink, 2,4 t Blei und 0,042 t Cadmium zu rechnen (SIEWERS \& ROOSTAI 1990). 


\section{1:3 Hydrographie}

\subsubsection{Abfluß}

Mit Ausnahme von Großer Bode und Warmer Bode kann das Abflußgeschehen der Untersuchungsbäche direkt anhand von Pegelschreiberaufzeichnungen der Harzwasserwerke nachvollzogen werden. Im Sösetal steht ein Pegelschreiber in der Ortschaft Riefensbeek an der Söse unterhalb des Zuflusses der Alten Riefensbeek. Ein zweiter befindet sich an der Großen Schacht unterhalb des Zusammenflusses mit der Rauhen Schacht. Die Pegelschreiber an der Langen Bramke und am Varleybach stehen unmittelbar unterhalb der Probestellen L2 bzw. V2.

In der Sösemulde kommen von der durchschnittlichen jährlichen Niederschlagsmenge von $1310 \mathrm{~mm} / \mathrm{m}^{2} 815 \mathrm{~mm}$ zum Abfluß (=62,2\%), von denen $63,4 \%$ im Winterhalbjahr und bei etwa gleicher Niederschlagsmenge aufgrund der höheren Verdunstung nur 36,6 \% im Sommerhalbjahr abfließen. Am Pegel Riefensbeek wurde für die Jahre 1941-1965 ein mittlerer Abfluß von $0,61 \mathrm{~m}^{3} / \mathrm{s}$ ermittelt. Am Pegel Schacht lag der mittlere Abfluß bei $0,27 \mathrm{~m}^{3} / \mathrm{s}$. Die Abflußspende betrug für beide Einzugsgebiete rund $28 \mathrm{l} / \mathrm{s}^{*} \mathrm{~km}^{2}$ (HAASE et al. 1970).

In der Zeit von April bis November 1987 wurden in einer Meßreihe mittels Drehflügelmessungen die Abflüsse von Großer Söse und Alter Riefensbeek getrennt untersucht (SIEBERT 1988). Diesen Daten ist zu entnehmen, daß der Abfluß aus dem Einzugsgebiet der Großen Söse einen Anteil von fast $58 \%$ am Gesamtabfluß der Söse bei mittlerem Wasserstand hat und die Alte Riefensbeek nur $25 \%$ beiträgt (Tab. 5). Bei Niedrigwasser verschiebt sich dieses Verhältnis geringfügig weiter zugunsten der Großen Söse. In Bezug auf die absoluten Wassermengen erreicht der mittlere Abfluß an allen Probestellen der Alten Riefensbeek weniger als die Hälfte des Abflusses an den entsprechenden Stellen der Großen Söse. Der mittlere Abfluß der Großen Schacht ist etwa halb so hoch wie der der Großen Söse.

Bei Niedrigwasser kann der Abfluß in allen drei Einzugsgebieten auf weniger als $20 \%$ des mittleren Abflusses zurückgehen, wobei an R3 mit nur noch 16,7\% der niedrigste Wert erreicht wird. Demgegenüber stehen Hochwässer, die die große Schwankungsbreite der Wassermengen in den Bachläufen besonders deutlich machen. An 60 Tagen des Jahres kann mehr als die Hälfte der jährlichen Wassermenge abfließen, wobei extreme Hochwasserwellen, die insbeson- 
Tab. 5: Abflußkenngrößen der Probestellen (nach SIEBERT 1988)

\begin{tabular}{rrrrr} 
Prbst. & $\begin{array}{c}\text { Mittlerer } \\
\text { Abfluß } \\
{[1 / \mathrm{s}]}\end{array}$ & \% Pegel & $\begin{array}{c}\text { Niedrigwasser- } \\
\text { abfluß } \\
\text { Il/s }]\end{array}$ & $\%$ Pegel \\
\hline R1 & 10,3 & 1,3 & 1,7 & 1,2 \\
R2 & 112,9 & 14,4 & 18,8 & 13,0 \\
R3 & 194,9 & 24,9 & 32,5 & 22,5 \\
S1 & 42,2 & 5,4 & 8,0 & 5,5 \\
S2 & 158,1 & 20,2 & 30,0 & 20,7 \\
S3 & 451,2 & 57,7 & 85,8 & 59,3 \\
M & 33,1 & 9,1 & 6,6 & 3,0 \\
SC & 224,5 & 61,6 & 44,5 & 20,5 \\
\hline
\end{tabular}

Tab. 6: Abflußkenndaten der Pegel in $\mathrm{m}^{3} / \mathrm{s}$

\begin{tabular}{lccccc}
\multicolumn{1}{c}{ Pegel } & Abflußjahre & $\bar{x}$ & $\pm s$ & Min & Max \\
\hline Riefensbeek & $1986-1989$ & 0,8136 & 1,1182 & 0,0550 & 12,3000 \\
Große Schacht & $1986-1989$ & 0,3166 & 0,4980 & 0,0100 & 6,9800 \\
Lange Bramke & $1986-1989$ & 0,0149 & 0,0212 & 0,0010 & 0,2730 \\
Varleybach & $1986-1988$ & 0,0278 & 0,0145 & 0,0140 & 0,1390
\end{tabular}

dere bei der Schneeschmelze in mehreren Phasen auftreten können, in der Lage sind, innerhalb von 24 Stunden $10 \%$ des mittleren Jahresabflusses zu liefern (HAASE et al. 1970). Ausgehend von den Daten der Pegelschreiberaufzeichnungen beträgt für die Wasserwirtschaftsjahre 1986-1989 am Pegel Riefensbeek die Schwankungsbreite zwischen Niedrigwasserabfluß und dem höchsten Wasserstand $6,8 \%$ bis $1512 \%$ vom mittleren Abfluß (Tab. 6). Am Pegel Große Schacht sind die Werte mit 3,2 \% und $2205 \%$ noch extremer. Das Bild in der Langen Bramke ist entsprechend $(6,7 \%-1838 \%)$. Eine deutliche Ausnahme macht der Varleybach, wo der Niedrigwasserabfluß immer noch 50,3\% des mittleren Abflusses erreicht und auch der höchste Wasserstand nur bei $499 \%$ des mittleren Abflusses liegt.

Aus den Abflußkurven ist abzulesen, daß alle Gebiete aufgrund der morphologischen und hydrogeologischen Situation sehr schnell auf Niederschläge reagieren 
44
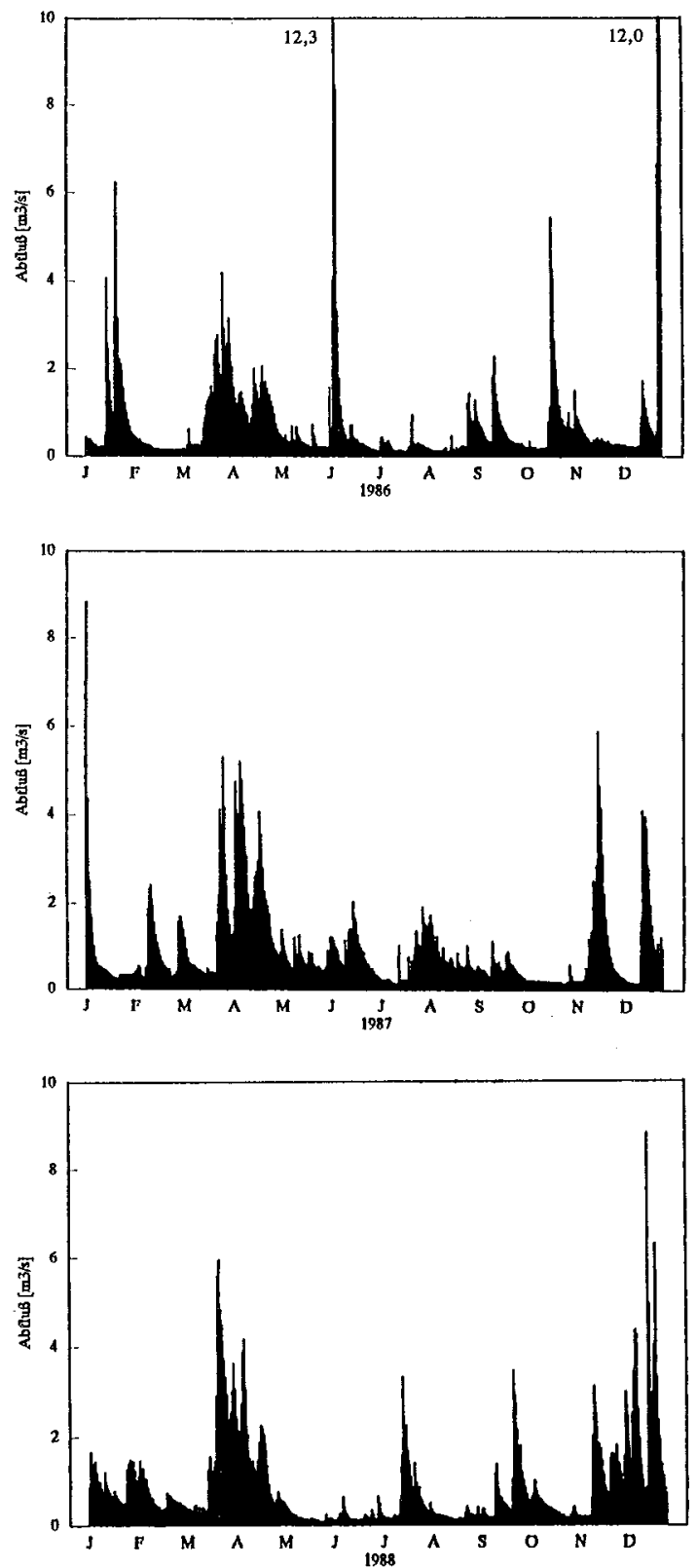

Abb. 14 a-c: Mittlere Tagesabflüsse am Pegel Riefensbeek in den Jahren 1986 bis 1988 

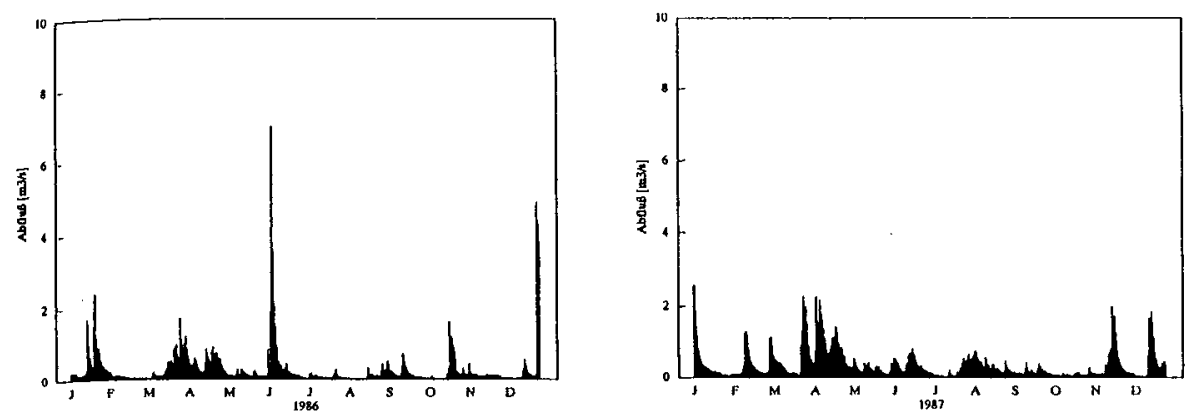

Abb. 15 a, b: Mittlere Tagesabflüsse am Pegel Große Schacht in den Jahren 1986 und 1987
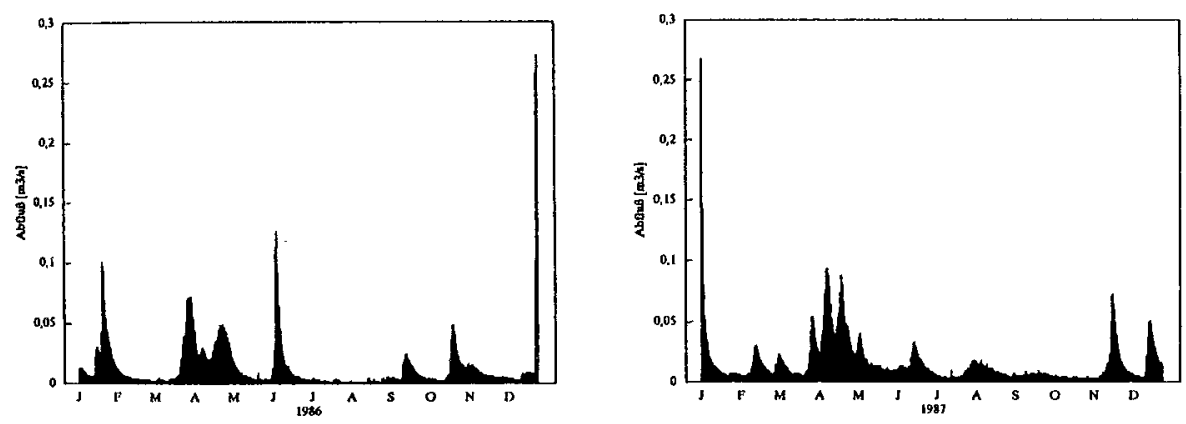

Abb. 16 a, b: Mittlere Tagesabflüsse am Pegel Lange Bramke in den Jahren 1986 und 1987 

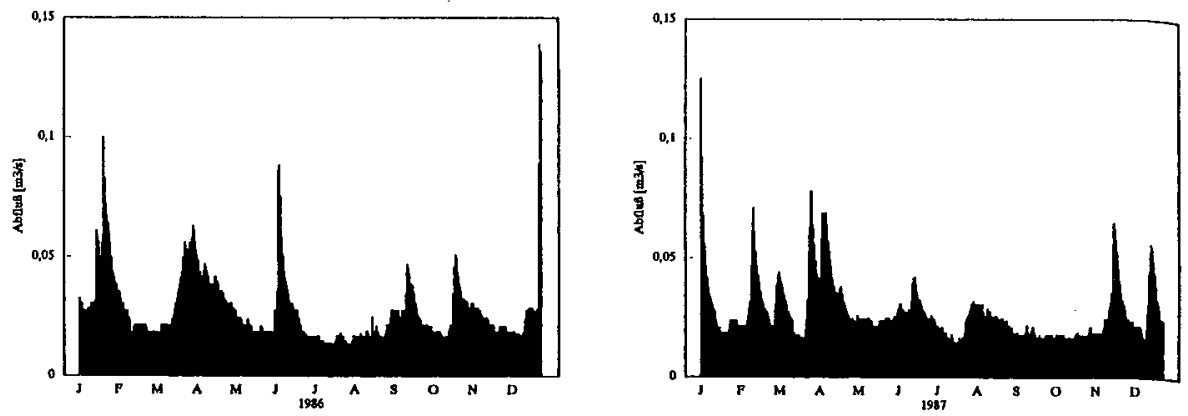

Abb. 17 a, b: Mittlere Tagesabflüsse am Pegel des Varleybachs in den Jahren 1986 und 1987

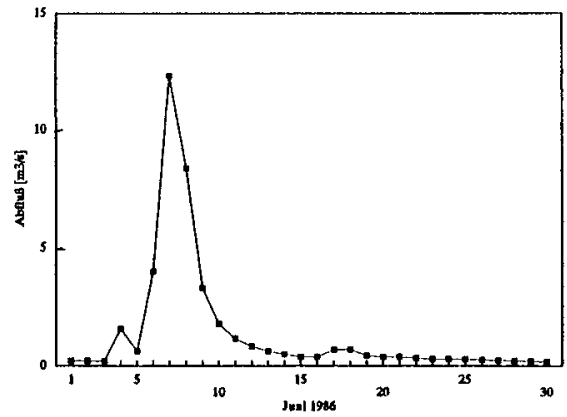

Abb. 18: Abflußkurve des Pegels Riefensbeek während eines Hochwassers im Juni 1986

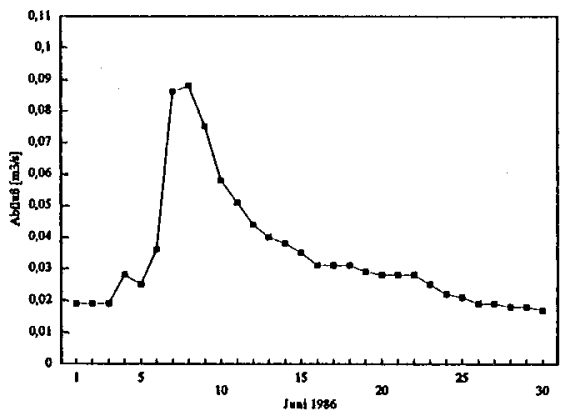

Abb. 19: Abflußkurve des Pegels am Varleybach während eines Hochwassers im Juni 1986 
und dementsprechend alle Abflußkurven einen ähnlichen Verlauf mit steilem Anstieg in kurzer Zeit zeigen (Abb. 14-17). Abgesehen vom Varleybach, in dem auch die Hochwasserspitzen wesentlich niedriger ausfallen als in den anderen Bächen, geht der Wasserstand nach dem Nachlassen der Niederschläge innerhalb weniger Tage wieder auf das Ausgangsniveau zurück (Abb. 18). Im Varleybach benötigt dieser Rückgang hingegen einen deutlich längeren Zeitraum (Abb. 19). Das Einzugsgebiet ist unter anderem auch aufgrund seines noch dichten Waldbestandes hydrologisch wesentlich besser gepuffert als die restlichen untersuchten Gebiete.

Während des Untersuchungszeitraums traten mehrere extreme Abflußsituationen auf. 1986 folgte einem kleineren Januarhochwasser ein allgemein recht hoher Wasserstand in den Monaten April und Mai. Anfang Juni kam es nach mehrtägigen, starken Regenfällen zu einem extremen Hochwasser, das an allen Pegeln abzulesen war. Ende Dezember 1986 / Anfang Januar 1987 folgte, ausgelöst durch eine Schneeschmelze mit kräftigen Niederschlägen, ein zweites extremes Hochwasser. Der Rest des Jahres brachte immer wieder höhere Wasserstände ohne längeren Niedrigwasserstand, durch den sich hingegen insbesondere das Jahr 1988 auszeichnete.

\subsubsection{Gefälle}

Die Quellgebiete der untersuchten Bäche liegen in Höhen zwischen $590 \mathrm{~m}$ (Varleybach) und $850 \mathrm{~m}$ (Große Bode). Vergleicht man die Gefällekurven der verschiedenen Bäche miteinander (Abb. 20, Tab. 7), zeigt sich, daß der Große Mollenbach bis zur Probestelle $M$ das höchste Gefälle aufweist $(31,4 \%)$. Für die Große Söse errechnet sich für die Strecke bis zur Probestelle S1 ein Gefälle von $25,7 \%$. Beide Bäche besitzen unterhalb der beiden Probestellen einen weitestgehend parallelen Verlauf der Kurven. Gegenüber den vom Acker kommenden Bächen ist die Gefällekurve der Alten Riefensbeek wesentlich ausgeglichener. Varleybach und Lange Bramke weisen dagegen einen deutlich steileren ersten Bachkilometer auf. Einen gänzlich anderen Verlauf zeigt die Gefällekurve von Großer Bode/Warmer Bode, die mit einem durchschnittlichen Gefälle von 4,2\% sehr flach ist. Dabei zeigen insbesondere die ersten drei Bachkilometer, bedingt durch die Lage des Quellgebietes auf einer Hochfläche, einen für Bergbäche ungewöhnlich flachen Verlauf. 


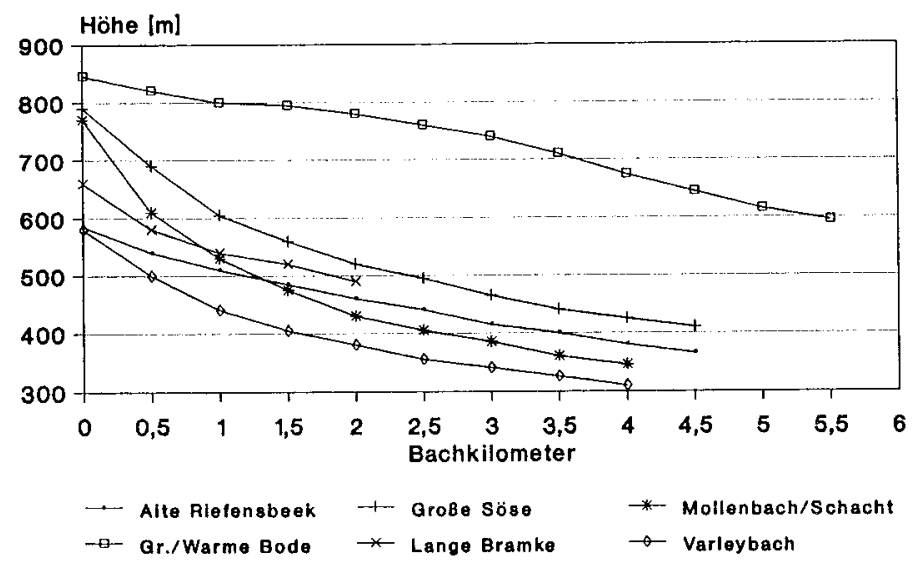

Abb. 20: Gefällekurven (zur Lage der Probestellen vgl. Tab. 7)

Abgesehen von den Bode-Probestellen, die $200 \mathrm{~m}$ über den quellnächsten Stellen der anderen Bäche liegen, beträgt der Höhenunterschied zwischen den vergleichbaren Probestellen der Untersuchungsgewässer nicht mehr als $100 \mathrm{~m}$ (Tab. 7), wobei die jeweils erste Probestelle sich, abgesehen von Varleybach $(1,3 \mathrm{~km})$ und Großer Bode $(2,5 \mathrm{~km})$, innerhalb des ersten Bachkilometers befindet und damit in den von einer möglichen Versauerung besonders betroffenen quellnahen Bereichen.

\subsubsection{Tiefe und Breite}

Bei mittleren Wasserständen sind die Unterschiede in der Wassertiefe zwischen den einzelnen Probestellen, abgesehen von B1 und L1, gering (Tab. 7). Die Große Bode weist an B1 auf längeren Strecken teilweise über $1 \mathrm{~m}$ hohe Uferkanten auf, zwischen denen der gesamte Gewässerboden auch bei Niedrigwasser stets überspült ist. Die Lange Bramke fließt an L1 durch einen breiten Moosstreifen, der sie fast vollständig einschließt und auch die Uferkanten bildet.

Teilweise große Unterschiede bestehen bedingt durch den stark unterschiedlichen Abfluß hinsichtlich der Gewässerbreite (Tab. 7), was wiederum Fließgeschwindigkeit und Substratzusammensetzung stark beeinflußt. Große Bode/ 
Tab. 7: Hydrographische Kenndaten der Probestellen

Halbquantitative Angabe der Fließgeschwindigkeiten für die Schwankungsbreite zwischen Niedrigwasserabfluß und Hochwässern: 1 stehend bis sehr langsam fließend, 2 langsam fließend, 3 schnell fließend, 4 sehr schnell fließend, 5 sturzbachartig fließend

\begin{tabular}{|c|c|c|c|c|c|c|c|c|c|}
\hline Prbst. & $\begin{array}{l}\text { Höhe } \\
\text { [m] }\end{array}$ & $\begin{array}{c}\text { Quell- } \\
\text { entf.[km] }\end{array}$ & $\begin{array}{c}\text { Gefälle } \\
{[\%]}\end{array}$ & $\begin{array}{c}\text { Breite } \\
{[\mathrm{m}]}\end{array}$ & $\begin{array}{l}\text { Tiefe } \\
{[\mathrm{cm}]}\end{array}$ & $\begin{array}{l}\text { Bach- } \\
\text { formation }\end{array}$ & $\begin{array}{c}\text { Fließ- } \\
\text { geschw. }\end{array}$ & $\begin{array}{l}\text { Geschiebe- } \\
\text { führung }\end{array}$ & $\begin{array}{l}\text { Gesteins- } \\
\text { formen }\end{array}$ \\
\hline R1 & 540 & 0,6 & 6 & $0,6-1,0$ & 10 & 11 & $2-3$ & gering & kantig(-rund) \\
\hline R2 & 450 & 2,1 & 4 & $2-3$ & 15 & 11 & $2-4$ & mäßig & kantig(-rund) \\
\hline R3 & 380 & 3.7 & 3 & $2-3$ & 20 & $1^{\prime}$ & $2-4$ & mäßig & $\begin{array}{l}\text { kantig-rund, } \\
\text { tlw. flächig }\end{array}$ \\
\hline s1 & 610 & 0,9 & 16 & 2 & 10 & $1^{\prime}$ & $1-5$ & hoch & kantig-rund \\
\hline s2 & 510 & 2,2 & 5 & $4-5$ & 10 & $1^{\prime}$ & 1.5 & hoch & kantig-rund \\
\hline s3 & 420 & 4,2 & 3 & $4-6$ & 20 & $2^{\prime}$ & $2-5$ & hoch & $\begin{array}{l}\text { rund-kantig, } \\
\text { t|w. flächig }\end{array}$ \\
\hline$M$ & 550 & 0,7 & 16 & 2 & 10 & $1^{\prime}$ & $2-5$ & hoch & kantig \\
\hline SC & 350 & 3,9 & 3 & $3-7$ & 15 & $2^{\prime}$ & $2-5$ & hoch & kantig-rund \\
\hline$B 1$ & 770 & 2,5 & 4 & $2-4$ & 25 & 1 ' & $2-5$ & hoch & kantig \\
\hline B2 & 600 & 5,5 & 4 & $6-8$ & 15 & $2^{\prime}$ & $3-5$ & hoch & rund-kantig \\
\hline L1 & 580 & 0,4 & 8 & 0,5 & $10-50$ & 1 ' & $2-3$ & gering & kantig-rund \\
\hline L2 & 540 & 1,0 & 6 & $0,5-1,0$ & 10 & 1 & $2-3$ & gering & kantig(-flächig) \\
\hline L3 & 490 & 1.9 & 5 & $1-2$ & 10 & $1^{\prime}$ & $2-4$ & gering & $\begin{array}{l}\text { rund-kantig, } \\
\text { tlw. flächig }\end{array}$ \\
\hline $\mathrm{V}_{1}$ & 410 & 1,3 & 7 & $1,5-2,0$ & 10 & $1^{\prime}$ & $2-4$ & mäßig & kantig-flächig \\
\hline v2 & 310 & 3,8 & 3 & 3 & 10 & $1 '$ & $2-4$ & mäßig & flächig-rund \\
\hline
\end{tabular}

Warme Bode, Große Söse und Großer Mollenbach/Große Schacht weisen an den von der Quellentfernung vergleichbaren Probestellen wesentlich größere Breiten auf (maximal $6-8 \mathrm{~m}$ ) als die kleineren Bäche Alte Riefensbeek, Lange Bramke und Varleybach (bis maximal $3 \mathrm{~m}$ ).

Die unteren Probestellen der drei größeren Gewässer stellen als einzige untersuchte Bereiche Bachformationen zweiter Ordnung dar (Tab. 7). Alle anderen Stellen gehören aufgrund der Abflußverhältnisse Gewässern erster Ordnung an (OTTO \& BRAUKMANN 1983). Mit einer mittleren Breite $>5 \mathrm{~m}$ ist B2 nach HUET (1949) bereits als Fluß zu klassifizieren. Da der mittlere Abfluß aber an dieser Stelle unter $5 \mathrm{~m}^{3} / \mathrm{s}$ liegt, kann auch die Warme Bode noch als Bach angesprochen werden (OTTO \& BRAUKMANN 1983). 


\subsubsection{Fließgeschwindigkeit und Morphologie}

Die aus Abbildung 21 zu entnehmenden Daten spiegeln die Fließgeschwindigkeiten an den repräsentativen Punkten der faunistischen Probenahmen wider. Sie vermitteln ein Bild von den vorherrschenden mittleren Fließgeschwindigkeiten bei mittleren und niedrigen Wasserständen. Abgesehen von $S 1$ mit $28 \mathrm{~cm} / \mathrm{s}$ und L1 mit $16 \mathrm{~cm} / \mathrm{s}$ liegen alle anderen Probestellen mit mittleren Fließgeschwindigkeiten zwischen 31 und $45 \mathrm{~cm} / \mathrm{s}$ relativ dicht beieinander. Mit zunehmender Quellentfernung ist in allen Bächen ein Anstieg der Fließgeschwindigkeiten zu verzeichnen. Bei den Maxima fallen die relativ niedrigen Werte der Langen Bramke, dem kleinsten der untersuchten Bäche, an L1 und L2 auf.

In der Regel ist in allen Bächen ein breites Spektrum an Fließgeschwindigkeiten dicht nebeneinander zu finden. Stillwasserzonen im Uferbereich stehen hohe Fließgeschwindigkeiten an Riffles auch bei Niedrigwasser gegenüber. Nur bei Hochwässern, wenn der Bach über die Ufer tritt, wird das gesamte Bachbett von den dann sehr hohen bis sturzbachartigen Fließgeschwindigkeiten erfaßt. Bei Hochwasser können sich insbesondere Große Söse, Großer Mollenbach/Große Schacht und Große Bode/Warme Bode in reißende Sturzbäche verwandeln. Demgegenüber sind die Verhältnisse auch bei Hochwasserereignissen an Alter Riefensbeek, Varleybach und insbesondere Langer

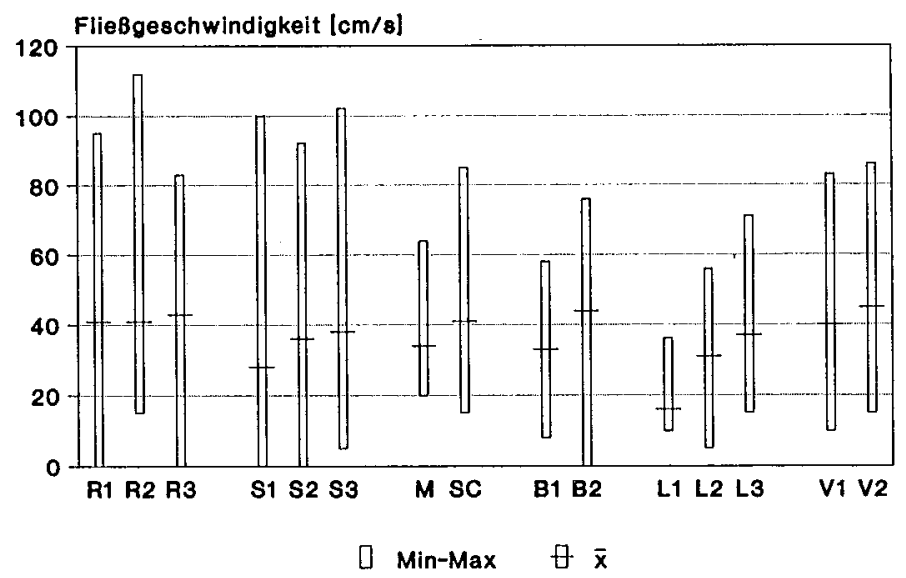

Abb. 21: Übersicht über die Fließgeschwindigkeiten zu den Zeitpunkten der Benthosbeprobungen 
Bramke ruhiger. Am größten ist der Unterschied zwischen Niedrig- und Hochwasserabfluß an S1 und S2, wo die Spannweite von fast stehenden geringen Wassermengen bis zu Sturzfluten reicht.

An R1, L1 - L3 und V2 ist bei normalen Wasserständen eine relativ gleichmäßige Strömung auf der gesamten Gewässerbreite zu finden. Teilweise liegt dies in der unterschiedlichen Ausprägung der für Bergbäche charakteristischen Riffle-PoolStruktur begründet, die besonders gut an den Probestellen R2, R3, S3, SC, B1, L1 und L2 zu sehen ist. Allgemein werden die Abstände zwischen Riffles und Pools bachabwärts abhängig von Abfluß und Gefälle größer. An R1, S1, S2, M und B2 ist die Struktur nur schwach entwickelt bzw. sie nimmt im Fall von $M$ und $\mathrm{S} 1$ aufgrund des starken Gefälles schon den kaskadenartigen Charakter von Gebirgsbächen an. An V2 ist keine Riffle-Pool-Struktur zu erkennen. Hieraus wird deutlich, wie schwierig es ist, die tatsächlichen Fließgeschwindigkeiten auch nur annähernd quantitativ zu beschreiben. Um aber dennoch ein Bild von den unterschiedlichen Verhältnissen zeichnen zu können, wurde versucht, die Spannweite der Fließgeschwindigkeit zwischen Niedrigwasserabfluß und Hochwasser halbquantitativ anzugeben (Tab. 7).

Sehr hohe Fließgeschwindigkeiten und eine instabile Bachsohle führen bei Hochwässern zu hohen Geschiebeführungen, wie sie in Großer Söse, Großem Mollenbach/Großer Schacht und Großer Bode/Warmer Bode festgestellt werden können (Tab. 7). Als gering muß hingegen die Geschiebeführung in Langer Bramke und im oberen Bereich der Alten Riefensbeek an R1 angesehen werden.

Die Stabilität der Bachsohle ist abhängig von Gesteinsformen und Korngrößen. Quellnah sind in allen Bächen vorwiegend mehr kantige Formen zu finden, die bachabwärts zu mehr runden Formen abgeschliffen werden. In den SchieferGebieten an Langer Bramke und Varleybach sowie an den untersten Stellen von Großer Söse und Alter Riefensbeek finden sich daneben auch flächige Formen (Tab. 7), die vom Wasser nur schlecht transportiert werden können. Man kann an diesen Stellen damit von einer höheren Sohlenstabilität ausgehen. Die von RÜDDENKLAU (1989) durchgeführte Untersuchung des Feinbodenmaterials erbrachte einen sehr niedrigen Anteil der feinsten Korngrößen in den BodeBächen, Großer Söse und Großer Schacht. Deutlich höher war er in Alter Riefensbeek, Varleybach und Langer Bramke. 


\subsubsection{Choriotopstruktur}

Bei der für die Besiedlung durch die Makrofauna wichtigen Zusammensetzung des Choriotopgefüges zeigen einige Probestellen starke Abweichungen von den restlichen Stellen, was sich durch eine Cluster-Analyse besonders gut verdeutlichen läßt (Tab. 8, Abb. 22). Danach unterscheiden sich L1, B1 und R1 von den anderen Probestellen. Dies liegt insbesondere begründet in dem an ihnen anzutreffenden relativ höheren Anteil an Psammal und Makropelal. Die restlichen Probestellen teilen sich in zwei weitere Gruppen auf, deren Unterschiede zueinander jedoch geringer sind als die zur ersten Gruppe. An R3, M, S1, S3, SC und B2 ist ein vergleichsweise höherer Anteil an Makrolithal zu finden als an R2, S2, L3, L2, V1 und V2, an denen an den meisten Stellen auch der Anteil des Akals höher liegt. Die Gruppenzuordnungen gründen sich auf Unterschiede im Gefälle, im Abfluß und in den Fließgeschwindigkeiten.
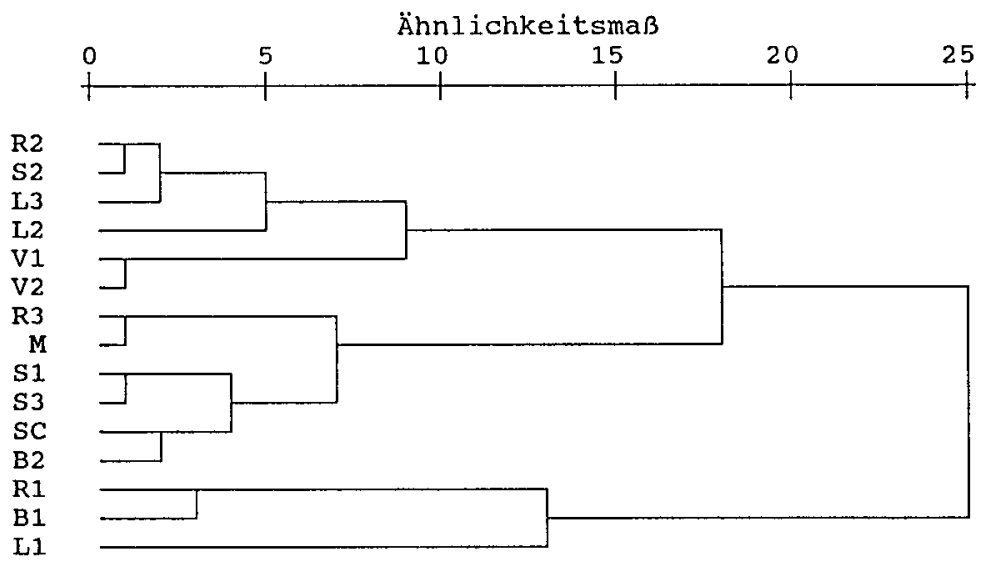

Abb. 22: Dendrogramm der Ähnlichkeit der Choriotopstrukturen zwischen den Probestellen als Ergebnis der Clusteranalyse nach dem WARDVerfahren 
Tab. 8: Choriotopstruktur der Probestellen

\begin{tabular}{|c|c|c|c|c|c|c|c|c|c|}
\hline Prbst. & $\begin{array}{c}\text { Cho } \\
1\end{array}$ & $\begin{array}{r}\text { otop } \\
2\end{array}$ & yp [ & 4 & 5 & 6 & 7 & 8 & 9 \\
\hline $\begin{array}{l}\text { R1 } \\
\text { R2 } \\
\text { R3 }\end{array}$ & $\begin{array}{r}<5 \\
15 \\
20\end{array}$ & $\begin{array}{r}5 \\
15\end{array}$ & $\begin{array}{l}20 \\
20 \\
10\end{array}$ & $\begin{array}{l}30 \\
30 \\
20\end{array}$ & $\begin{array}{l}20 \\
10 \\
10\end{array}$ & $\begin{array}{r}10 \\
5 \\
5\end{array}$ & $\begin{array}{r}<5 \\
5 \\
5\end{array}$ & - & $\begin{array}{l}15 \\
10 \\
15\end{array}$ \\
\hline $\begin{array}{l}\text { S1 } \\
\text { S2 } \\
\text { S3 }\end{array}$ & $\begin{array}{l}25 \\
20 \\
20\end{array}$ & $\begin{array}{l}30 \\
10 \\
20\end{array}$ & $\begin{array}{l}20 \\
20 \\
20\end{array}$ & $\begin{array}{l}15 \\
30 \\
15\end{array}$ & $\begin{array}{r}5 \\
10 \\
10\end{array}$ & $\begin{array}{r}<5 \\
5 \\
5\end{array}$ & $<5$ & - & $\begin{array}{l}<5 \\
<5 \\
10\end{array}$ \\
\hline $\begin{array}{l}M \\
S C\end{array}$ & $\begin{array}{l}25 \\
30\end{array}$ & $\begin{array}{l}15 \\
10\end{array}$ & $\begin{array}{l}15 \\
25\end{array}$ & $\begin{array}{l}15 \\
20\end{array}$ & $\begin{array}{l}5 \\
5\end{array}$ & $\begin{array}{l}<5 \\
<5\end{array}$ & $\begin{array}{l}<5 \\
<5\end{array}$ & - & $\begin{array}{l}20 \\
<5\end{array}$ \\
\hline $\begin{array}{l}\text { B1 } \\
\text { B2 }\end{array}$ & $\begin{array}{l}10 \\
20\end{array}$ & $\begin{array}{l}10 \\
15\end{array}$ & $\begin{array}{l}10 \\
25\end{array}$ & $\begin{array}{l}15 \\
10\end{array}$ & $\begin{array}{l}30 \\
15\end{array}$ & $\begin{array}{r}10 \\
5\end{array}$ & 5 & - & $\begin{array}{r}15 \\
5\end{array}$ \\
\hline $\begin{array}{l}\text { L1 } \\
\text { L2 } \\
\text { L3 }\end{array}$ & $\begin{array}{r}- \\
5 \\
20\end{array}$ & $\begin{array}{l}- \\
-\end{array}$ & $\begin{array}{r}5 \\
20 \\
10\end{array}$ & $\begin{array}{l}40 \\
45 \\
35\end{array}$ & $\begin{array}{r}30 \\
5 \\
15\end{array}$ & $\begin{array}{r}10 \\
5 \\
5\end{array}$ & $\begin{array}{r}15 \\
10 \\
5\end{array}$ & - & $\begin{array}{r}- \\
10 \\
10\end{array}$ \\
\hline $\begin{array}{l}\vee 1 \\
\vee 2\end{array}$ & $\begin{array}{r}10 \\
5\end{array}$ & - & $\begin{array}{l}30 \\
40\end{array}$ & $\begin{array}{l}25 \\
20\end{array}$ & $\begin{array}{r}5 \\
10\end{array}$ & $\begin{array}{r}5 \\
<5\end{array}$ & $\begin{array}{r}5 \\
<5\end{array}$ & - & $\begin{array}{l}20 \\
20\end{array}$ \\
\hline
\end{tabular}

Choriotop-Typen (nach BRAUKMANN 1987)

1 Makrolithal I

2 Makrolithal II

3 Mesolithal

4 Akal

5 Psammal

6 Makropelal

7 Mikropelal

8 Phytal

9 Lithophytal grobes Blockwerk, kopfgroße Steine (2/3 diese Korngröße, 1/3 Grob-, Kleinschotter und Kies)

Oberseite großer Steine, anstehender Fels

Grobschotter, handgroße Steine

(bis $1 / 3$ Kleinschotter, Kies, Sand)

Kleinschotter bzw. Grobkies, ei- bis haselnußgroß

(bis $1 / 3$ Grob- und Feinsand)

Feinkies und Sand (bis $1 / 3$ Schluff)

grobes organisches Material mit Kies und Sand feiner organischer Detritus mit Sand und Schluff submerse Wasserpflanzen

submerse epilithische Moos- und Algenpolster auf Steinen und Fels 
5.1.4 Gewässerrandvegetation, Primärproduktion und allochthone Nährstoffversorgung

Alle Probestellen befinden sich mehr oder weniger in einem der den Harz zu einem großen Teil bedeckenden Fichtenforste oder an einem Fichtenforstrand. Zur Vegetation am Gewässerrand gehören in der Regel bei nicht zu starker Beschattung eine Rasenhochstaudenflur sowie einzelne Sträucher (Tab. 10). Gewässerbegleitend treten außerdem an den meisten Probestellen einzelne Laubbäume auf. Gänzlich andere Vehältnisse sind an L1 anzutreffen, wo der Bach durch ein Quellmoor verläuft, und die Vegetation durch verschiedene Sphagnum-Arten, Flatterbinse und Waldschachtelhalm geprägt wird. Diese Arten treten an L2 ebenfalls noch auf.

Die Beschattung der Probestellen ist abhängig von der Lage zum Fischtenforst, dem Alter der Fichten und der Geländemorphologie. Hohe Bestände sind fast immer durchgeforstet und lichter als jüngere, wobei auch das Waldsterben zu einer Auflichtung führt. Die stärkste Beschattung ist an den Stellen R2, S2, S3, $L 2, L 3$ und $V 2$ zu verzeichnen. Relativ gering beschattet sind hingegen R1, S1, $M$ und L1 (Tab. 10).

Vom Grad der Beschattung ist die Primärproduktion im Gewässer abhängig. Eine submerse Bachvegetation ist an allen Probestellen ausgebildet (Tab. 10). Abgesehen von der normalen Jahresrhythmik kann sie zeitweilig nach Hochwasserwellen, die mit starken Substratumlagerungen einhergehen, verschwunden sein. Die größte Verbreitung zeigen in allen Gewässern Diatomeen und Moose, von denen die Diatomeen in einer eigenen Arbeit untersucht worden sind (CORING 1988, CORING \& HEITKAMP 1989). Moose sind an R1, R2, M, B1, $L 2, V 1$ und $V 2$ weit verbreitet, wobei die Zusammensetzung der Arten jedoch sehr unterschiedlich ist (Tab. 9). L1 ist von Moosen vollständig eingeschlossen. In den Sommermonaten können sich bei Niedrigwasserständen an einigen Probestellen Grünalgen gut entwickeln. So ist in der Großen Söse die Gattung Stigeocloneum in teilweise dichten Beständen zu finden. An M und B1 breiten sich hingegen verschiedene Ulothrix-Arten aus, die vereinzelt auch in der Alten Riefensbeek vorkommen (RÜDDENKLAU 1989). Als Vertreter der höheren Pflanzen wächst an R1 und R2 im Sommer und Herbst zeitweilig im Uferbereich die Bachgrundel (Peplis portula L.). 
Die für große Teile der Bachfauna wichtige Zufuhr von allochthonen Nährstoffen ist in hohem Maße abhängig von der Ufervegetation. Relativ groß ist die allochthone Nährstoffversorgung mit Laub, Nadeln, Fallholz, Grashalmen und z.T. auch Feindetritus an allen Probestellen der Alten Riefensbeek sowie an L2, V2 und B1, wobei an B1 Laub jedoch fehit (Tab. 10).

Tab. 9: An den Probestellen vorkommende aquatische Moose (aus RÜDDENKLAU 1989)

Häufigkeit: 1 sehr selten, 2 selten bis vereinzelt, 3 verbreitet, 4 häufig, 5 sehr häufig bis massenhaft

$$
\begin{array}{lllllllllllllll}
\text { A1 } & R 2 & R 3 & S 1 & S 2 & S 3 & M & S C & 81 & B 2 & L 1 & L 2 & L 3 & V 1 & V 2
\end{array}
$$

Brachythecium rivulare B.S.G.

Calliergonella cuspidata (HEDW.) Loeske

Chyloscyphus polyanthos (L.) Corda var. rivularis (SCHR.)

Pellia epiphylla (L.) Corda

Platyhypnidium riparoides (HEDW.) Dix.

Polytrichum commune (HEDW.)

Scapania undulata (L.) Dum.

Sphagnum spp.

$\begin{array}{lllllllllllllll}- & 1 & 1 & - & - & - & - & 1 & - & - & - & - & - & - & - \\ - & 1 & - & - & - & - & - & - & - & - & - & - & - & - & - \\ - & 1 & 2 & - & - & - & - & - & - & - & - & - & - & - & - \\ 1 & - & - & 1 & 1 & 3 & - & 1 & 3 & 2 & - & - & - & - & - \\ 4 & 4 & 3 & - & - & - & - & - & - & - & - & - & - & 5 & 5 \\ - & - & - & 2 & 1 & - & 3 & - & 4 & 3 & 5 & 4 & 3 & - & - \\ 1 & 2 & 2 & 2 & 2 & 2 & 5 & 1 & 5 & 3 & - & 3 & 3 & 1 & 2 \\ - & - & - & 1 & - & - & 1 & - & 2 & 1 & 5 & 2 & 2 & - & -\end{array}$




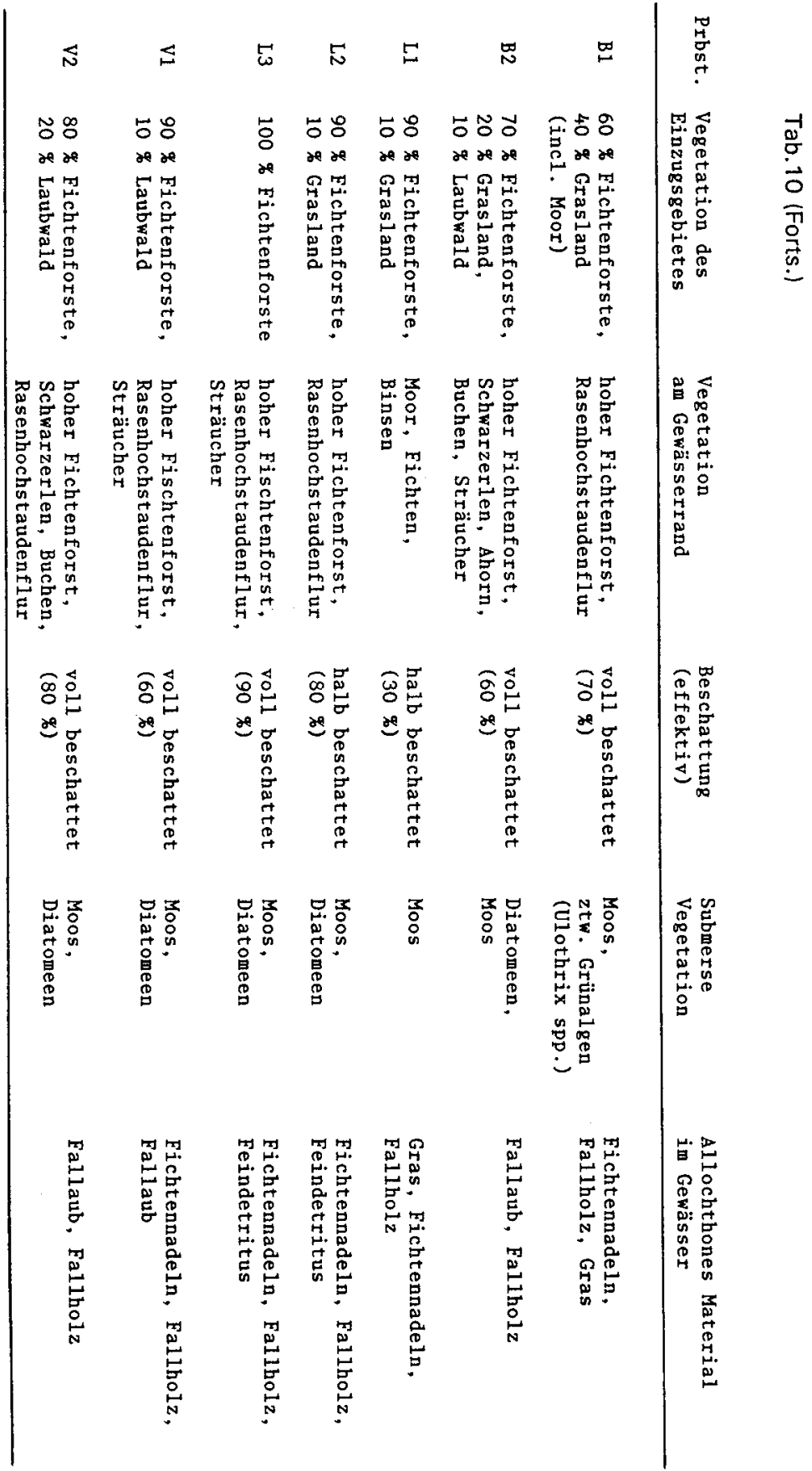




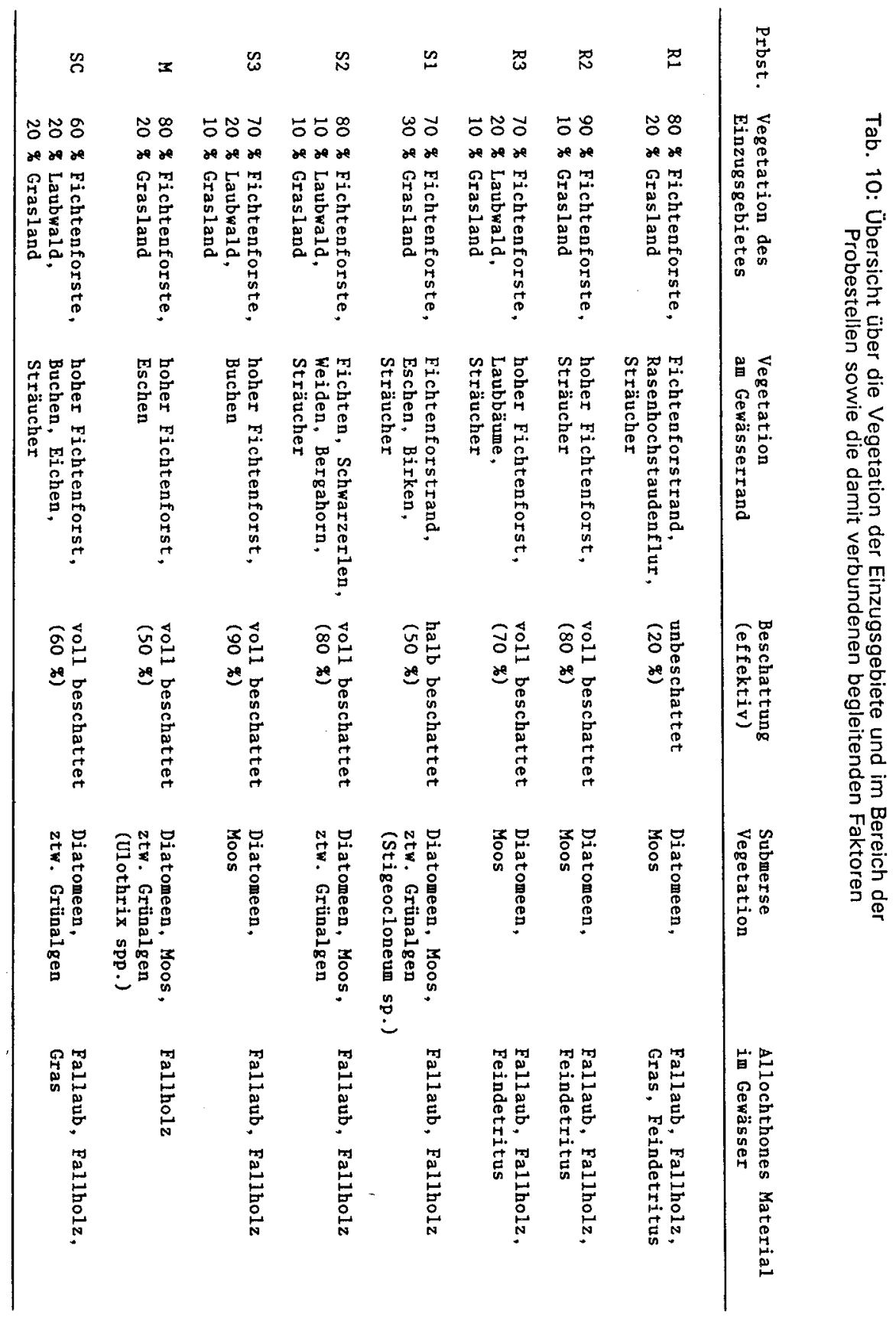




\subsubsection{Physikalisch-chemische Untersuchungsergebnisse}

5.1.5.1 Wasserstoffionenkonzentration

Da die vorliegenden Untersuchungen vor allem im Hinblick auf die Auswirkungen der Gewässerversauerung durchgeführt worden sind, stellen die Wasserstoffionenkonzentrationen an den einzelnen Probestellen den Angelpunkt der Betrachtungen der physikochemischen Lebensbedingungen für die Makrofauna dar.

Zwischen 1986 und 1988 wurden an allen Probestellen von Alter Riefensbeek und Großer Söse mehr als $100 \mathrm{pH}-$ Messungen durchgeführt, von den anderen Stellen liegen jeweils mehr als 60 Messungen vor (Tab. 11, Abb. 25 - 30). Um die Probestellen herauszuarbeiten, die ein ähnliches bzw. voneinander stark abweichendes Verhalten der $\mathrm{pH}$-Werte zeigen, wurden die Daten von den 44 Messungen, die an gleichen oder aufeinanderfolgenden Tagen an allen Stellen gemacht wurden, einer Cluster-Analyse unterzogen. Nach dem Single-LinkageVerfahren setzen sich S1 und L1 deutlich als "Ausreißer" von den restlichen Probestelien ab. Das Dendrogramm der WARD-Analyse zeigt drei voneinander getrennte Hauptgruppen, von denen zwei in jeweils zwei Untergruppen aufgeteilt sind (Abb. 23):

$\begin{array}{ll}1 & \text { S1, L1 } \\ 2.1 & \text { B1, M } \\ 2.2 & \text { S2, B2, L2 } \\ 3.1 & \text { R1, R2, R3, V2 } \\ 3.2 & \text { L3, V1, S3, SC }\end{array}$

Eine der Hauptgruppen wird von den durch das Single-Linkage-Verfahren angezeigten "Ausreißern" S1 und L1 gebildet. Dies sind die beiden Probestellen der Untersuchung, an denen konstant niedrige $\mathrm{pH}$-Werte gemessen wurden. Der Mittelwert ist $<5,0$ und die Schwankungsbreite $<0,7$ Einheiten (Abb. 24). Eine Untergruppe der zweiten Hauptgruppe besteht aus den Probestellen S2, B2 und $L 2$, während $B 1$ und $M$ in der zweiten Untergruppe zu finden sind. Alle fünf Probestellen zeichnen sich durch große Schwankungen des $\mathrm{pH}$-Wertes zwischen neutral und sauer aus. In der ersten Untergruppe sind große Schwankungen jedoch relativ selten und der Mittelwert des $\mathrm{pH}$ liegt zwischen 5,0 und 6,0. Die beiden Probestellen $B 1$ und $M$ der zweiten Untergruppe weisen hingegen häufige große $\mathrm{pH}$-Sprünge auf und haben einen Mittelwert $<5,0$, so daß sie zwischen den Probestellen der zuerst genannten Hauptgruppe und den restlichen Probe- 

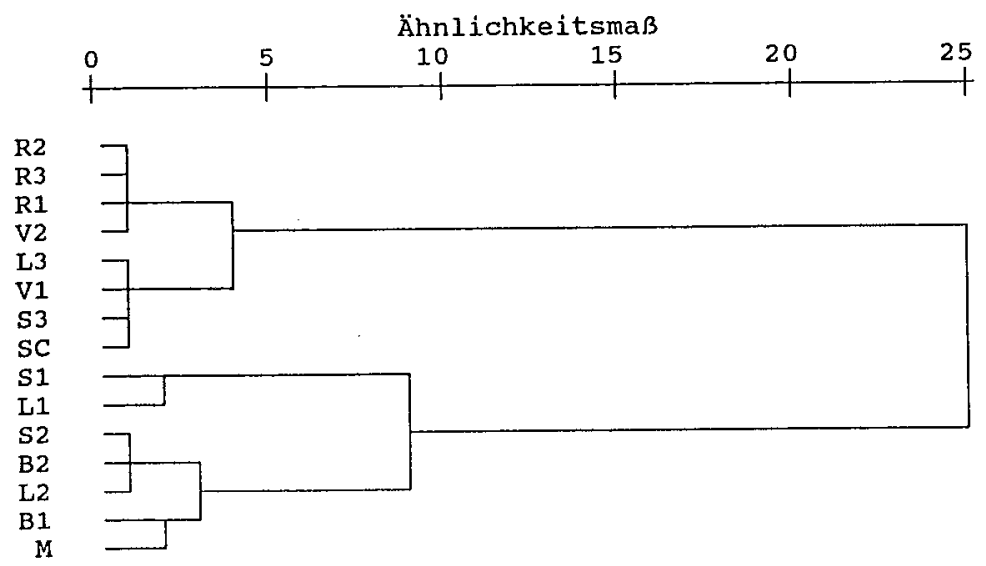

Abb. 23: Dendrogramm zur Cluster-Analyse der $\mathrm{pH}$-Werte nach dem WARDVerfahren

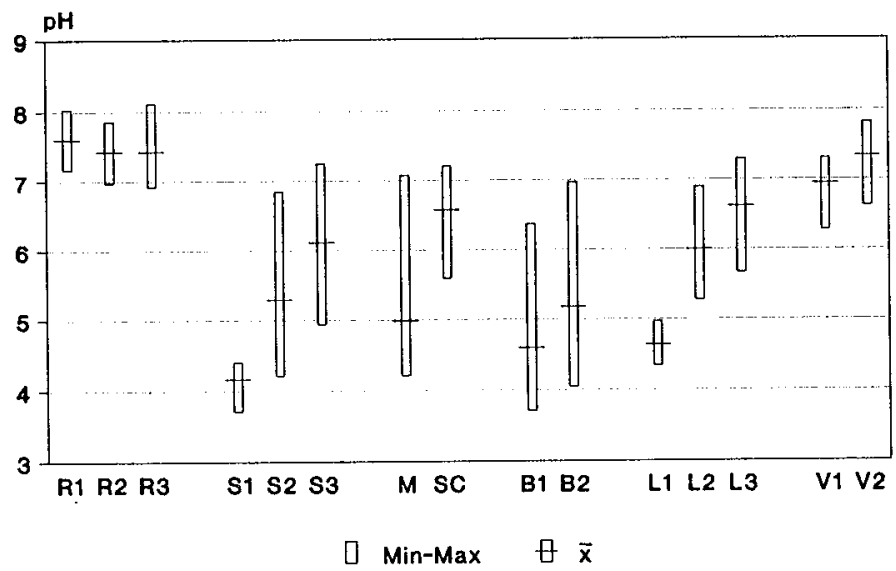

Abb. 24: Mittlere $\mathrm{pH}-$ Werte und Schwankungsbreite der $\mathrm{pH}-$ Werte an den Probestellen 
stellen der zweiten Hauptgruppe stehen. In der ersten Untergruppe der dritten Hauptgruppe sind die Probestellen der Alten Riefensbeek und V2 zu finden, in der zweiten Untergruppe L3, V1, S3 und SC. Alle Stellen haben einen Mittelwert $>6,0$, wobei in der ersten Untergruppe die Stellen zusammengefaßt sind, deren $\mathrm{pH}$-Wert bei relativ geringen Schwankungen konstant im neutralen Bereich liegt und deren Mittelwert > 7.0 ist. In der zweiten Untergruppe findet sich mit V1 sowohl eine Stelle, die zu dieser ersten Untergruppe aufgrund ebenfalls geringer $\mathrm{pH}$-Schwankungen auf aber insgesamt niedrigerem Niveau tendiert, als auch Stellen, die einen ähnlichen pH-Gang wie die zweite Hauptgruppe aufweisen. L3, S3 und SC zeigen jedoch nicht so stark ausgeprägte Sprünge zwischen neutral und sauer und bewegen sich dabei ausschließlich in einem schwächer sauren Bereich.

Bei Anordnung der Probestellen in der Reihenfolge der mittleren $\mathrm{pH}$-Werte und unter Berücksichtigung von Frequenz und Intensität der pH-Schwankungen lassen sich somit die Probestellen in folgender Weise gruppieren:

$\begin{array}{lll}1 & \text { S1, B1, L1, M } & \text { "stark versauert" } \\ 2 & \text { B2, S2, L2 } & \text { "mäßig versauert" } \\ 3 & \text { S3, SC, L3 } & \text { "schwach versauert" } \\ 4 & \text { V1, V2, R3, R2, R1 } & \text { "nicht versauert" }\end{array}$

Die durch Definition festgelegten Bezeichnungen für die Gruppen sind nur als Hilfsmittel bei der Unterscheidung zu verstehen, da die tatsächlichen pHVerhältnisse, wie dargestellt wurde, an den einzelnen Probestellen wesentlich differenzierter sind.

Der Zeitpunkt des Auftretens großer pH-Sprünge an B1, M, S2, B2, L2, L3, S3 und $\mathrm{SC}$ ist in starkem Maße abhängig vom Abfluß. Dies läßt sich an den Probestellen nachweisen, an denen sich Pegelschreiber in nicht zu großer Entfernung befinden (L2, SC, S3). Am Beispiel des Abflusses am Pegel Riefensbeek und der an $\mathrm{S} 2$ und $\mathrm{S} 3$ gemessenen $\mathrm{pH}$-Werte wird dies besonders deutlich (Abb. 31, 32). Die Große Söse trägt im Jahresdurchschnitt $58 \%$ zum Abfluß am Pegel bei. Zwischen Abflußwerten und pH-Werten errechnet sich ein Korrelationskoeffizient von $r=-0,73(p<0,001)$. PH-Sprünge treten eindeutig immer in Verbindung mit starken Abflußänderungen auf, wobei die höchsten Wasserstände die niedrigsten $\mathrm{pH}$-Werte aufweisen, ohne daß jedoch eine einfache lineare Beziehung besteht. Es ist die Tendenz zu erkennen, daß insbesondere 
das erste Hochwasser nach einer längeren Niedrigwasserperiode zu einem besonders starken $\mathrm{pH}-\mathrm{Sturz}$ führt. Diese stark ausgeprägte Beziehung zwischen Abfluß und $\mathrm{pH}$-Wert zeigt sich auch an L2 und SC. An V2 treten ebenfalls bei hohen Wasserständen niedrige $\mathrm{pH}$-Werte auf, die an dieser Stelle immer im neutralen Bereich liegen.
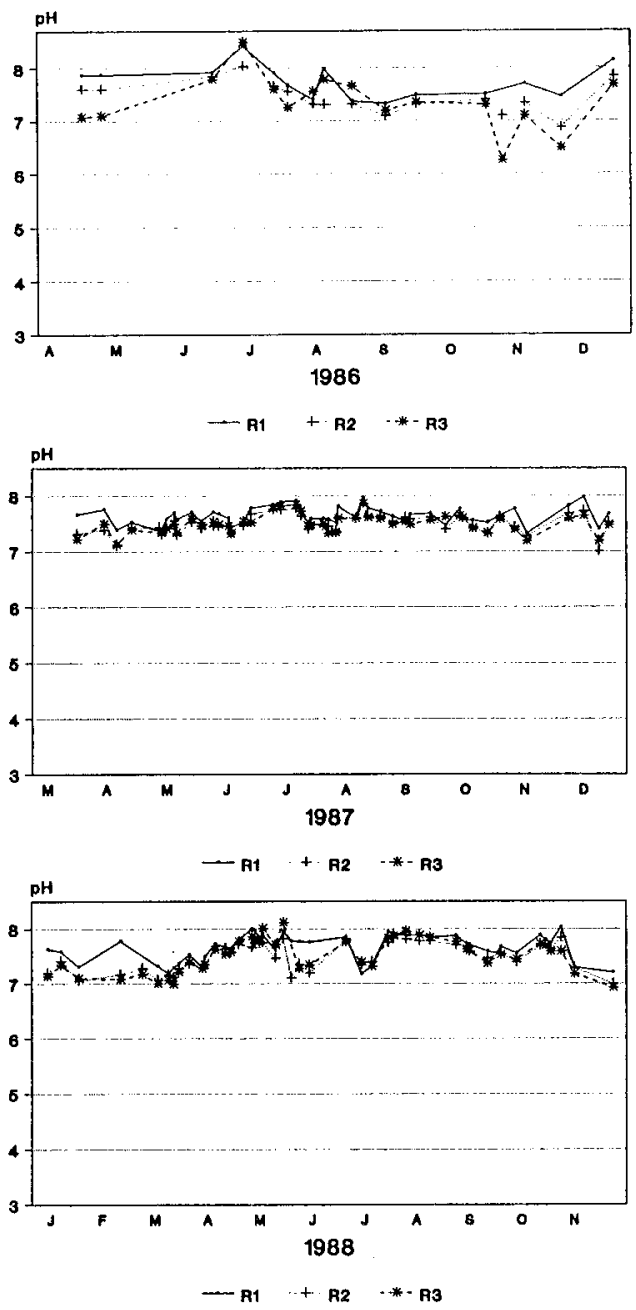

Abb. 25: Ganglinien der pH-Werte an den Probestellen der Alten Riefensbeek 

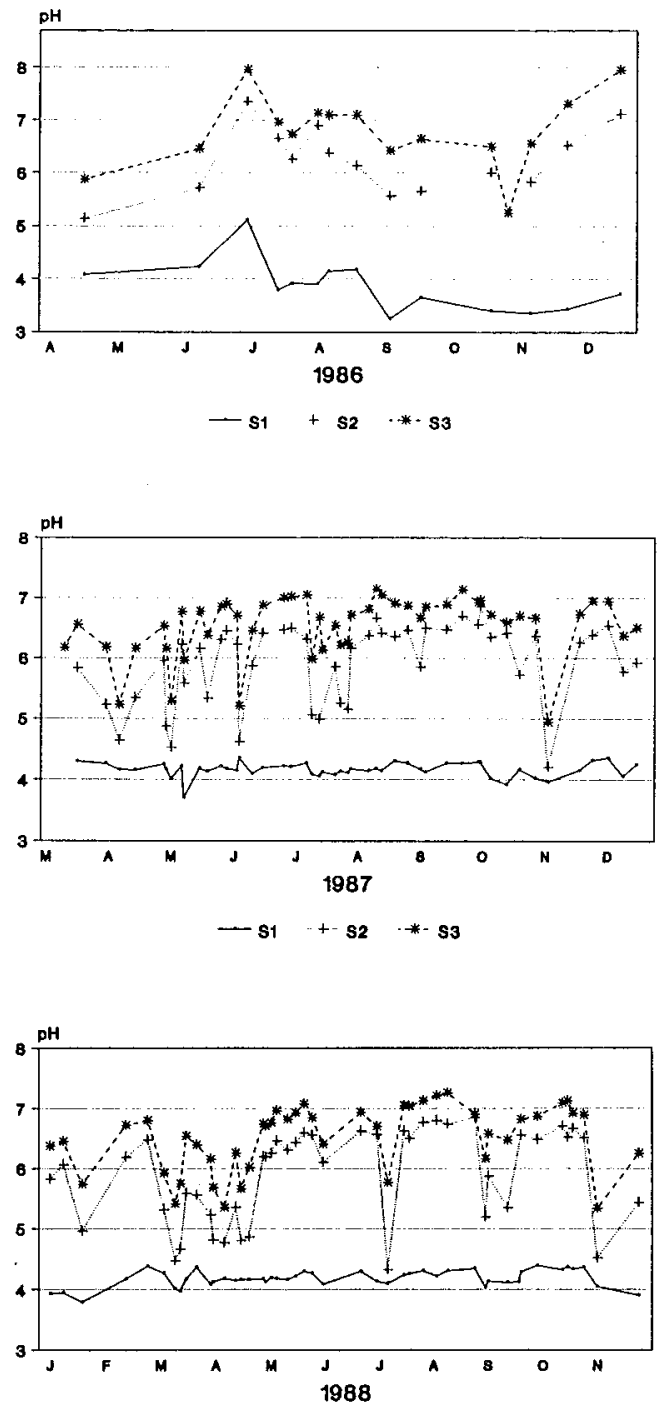

$-\$ 1+-\$ 2 \quad-*-\$ 3$

Abb. 26: Ganglinien der $\mathrm{pH}$-Werte an den Probestellen der Großen Söse 

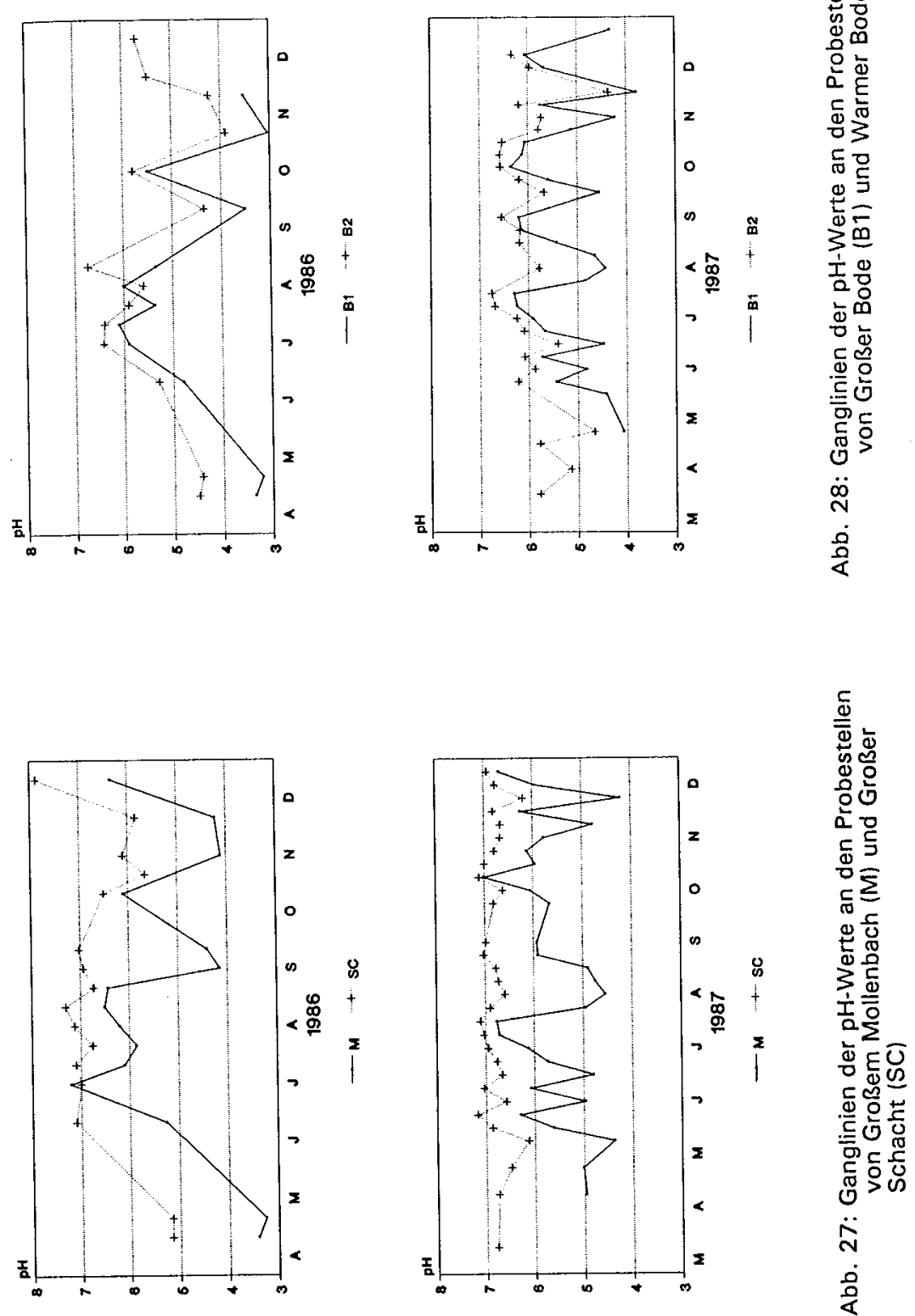

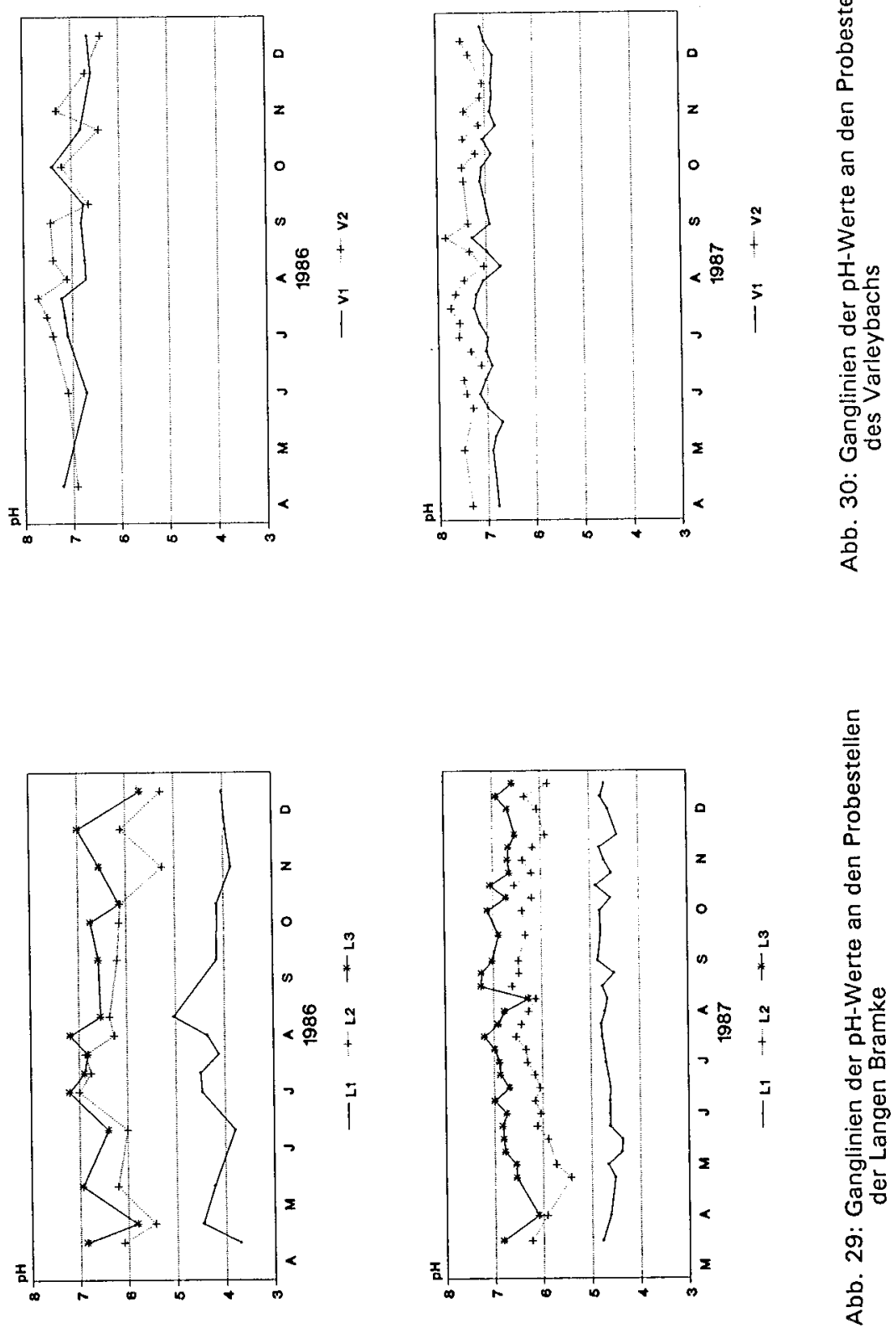


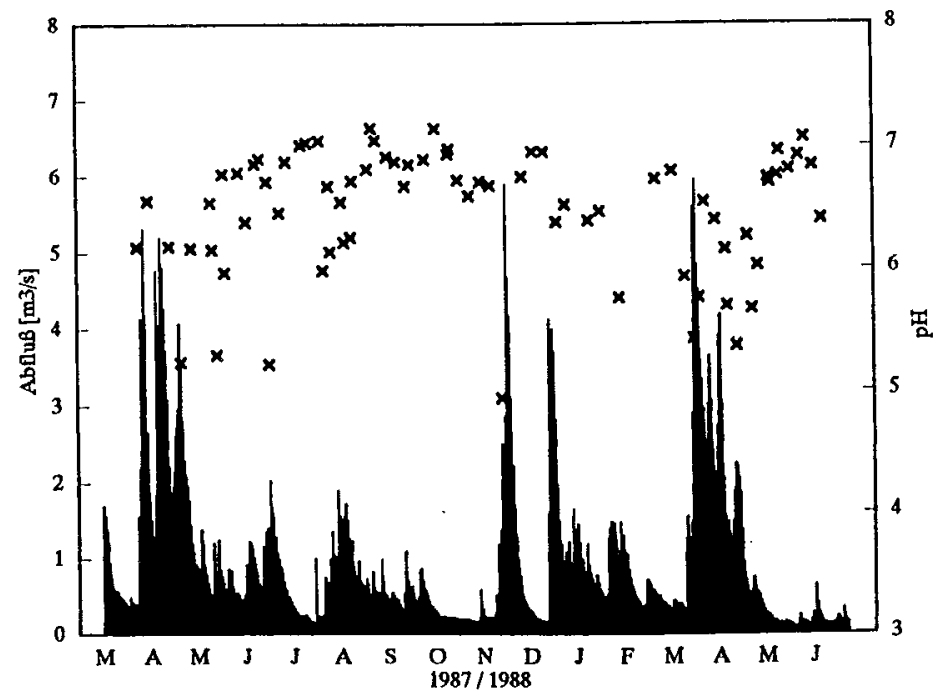

Abb. 31: Abfluß am Pegel Riefensbeek (Balken) und pH-Werte (x) an S3

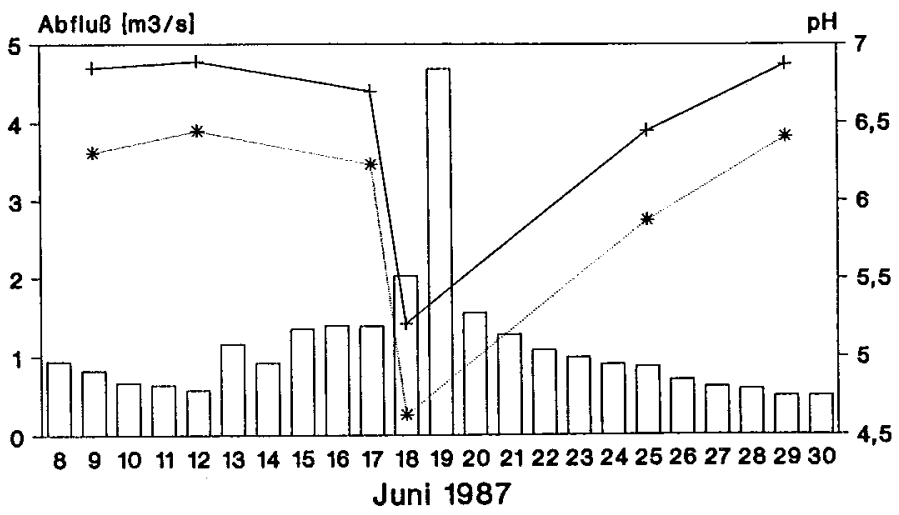

$\square$ mittl. Tages-Abfluß $\quad-1$ pH S3 $\quad \cdots$ *- $\mathrm{pH} \mathrm{S2}$

Abb. 32: Gang der pH-Werte an S2 und S3 während eines am Pegel Riefens beek registrierten Hochwassers (mittlerer Tagesabfluß) 


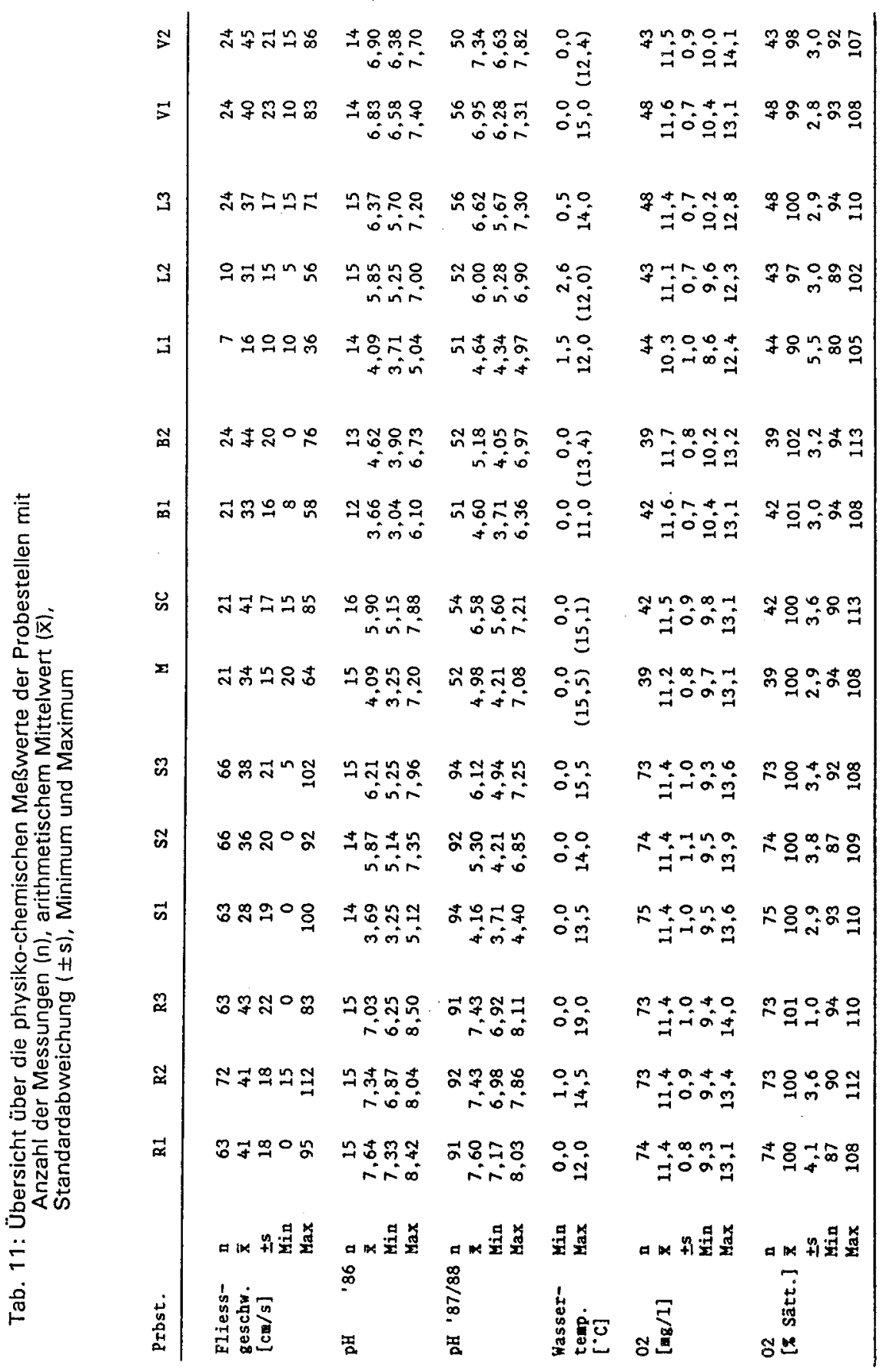




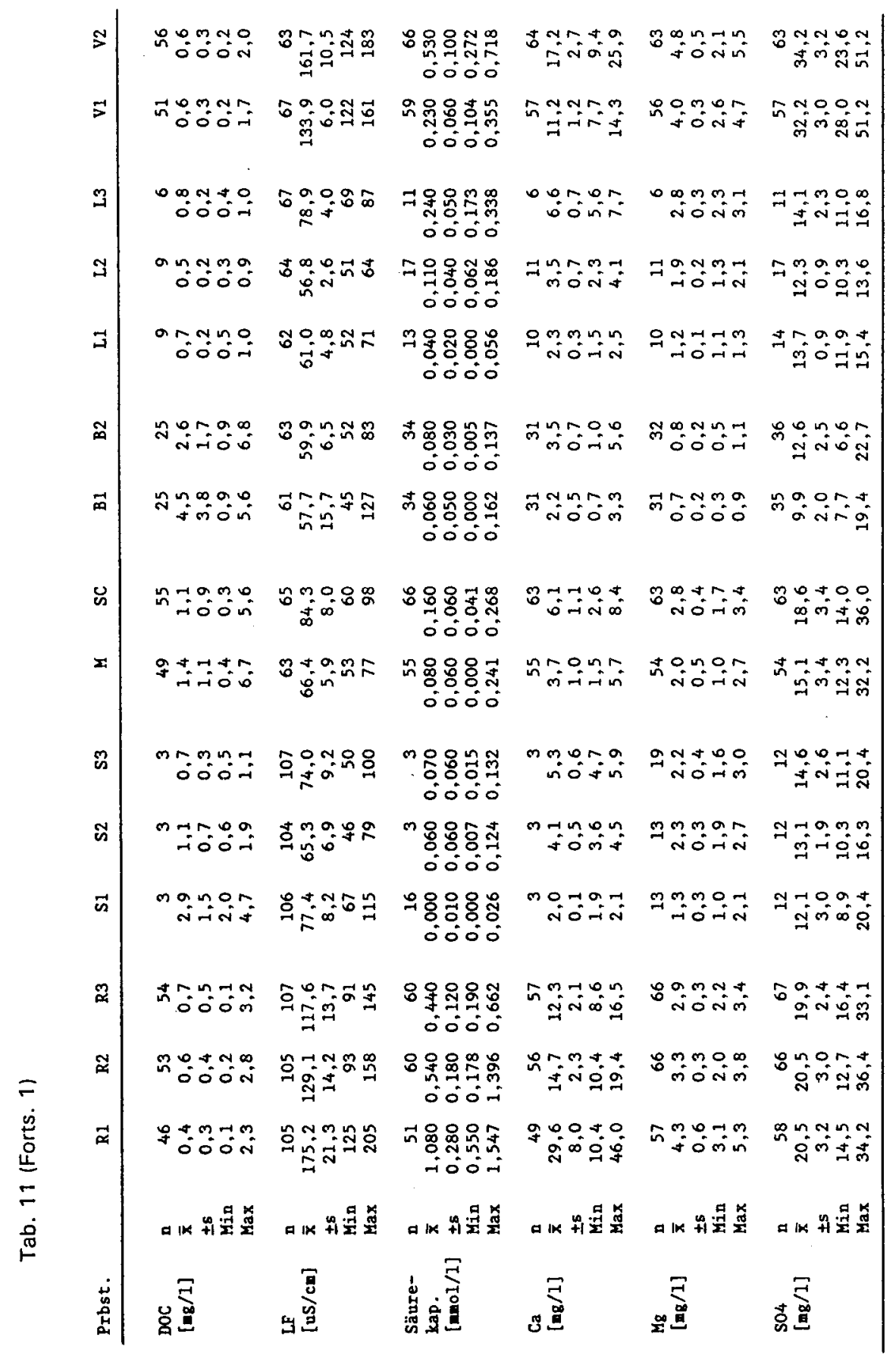




\begin{tabular}{|c|c|c|c|c|c|c|}
\hline 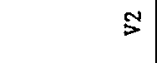 & $\begin{array}{l}+4 m a \\
0+n+a\end{array}$ & ถี่ & 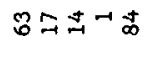 & $\stackrel{\infty}{\sim} \underset{\infty}{-\infty}+\infty$ & 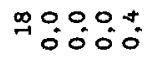 & 융요 \\
\hline 5 & 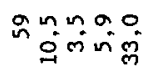 & ีㅗำ용 & ํํㅊ용 & $\because$ ก $\tilde{N}^{n}$ & $\begin{array}{l}n=0 \\
\Rightarrow 00 \\
0\end{array}$ & $\because 0: 00=$ \\
\hline 9 & 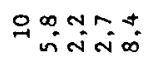 & ナ寻志声る゙ & $\exists \cong \infty \infty \mathrm{g}$ & 11111 & 11111 & 11111 \\
\hline ్ㅗ & 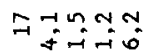 & 11111 & 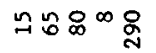 & N两, 早吉 & $\begin{array}{l}N N, N= \\
0\end{array}$ & 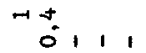 \\
\hline$\exists$ & 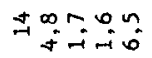 & 11111 & 공워 & N品, 8 & 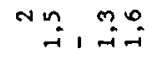 & $N m, \div$ \\
\hline$\underset{\infty}{\sim}$ & 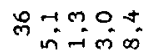 & 융ㅇㅇㅛ & 以 & 음융윰 & 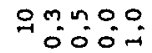 & $\begin{array}{l}\Rightarrow 000 \\
\Rightarrow 000\end{array}$ \\
\hline$\vec{\infty}$ & 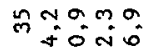 & 옹워구웅 & 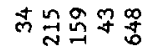 & ${ }^{a}$ 융요 & $\begin{array}{r}a+n o 0 \\
000-1\end{array}$ & $\begin{array}{r}0.000 \\
\text { Fi: }\end{array}$ \\
\hline$\ddot{n}$ & 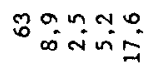 & 윶요믁 & 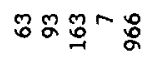 & $\pm \mathbb{N} \mathbb{N}^{\infty} \infty$ & 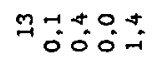 & $\pm \because \div 00$ \\
\hline$x$ & जी & $\exists \vec{\sigma} \infty \sim q$ & 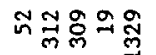 & 옥정원엄 & 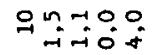 & 응응 \\
\hline $\mathscr{n}$ & 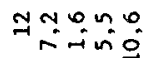 & Nล๊ N & 어웡웡요 & 옥독웅요 & 舟每: & m0 \\
\hline జ & 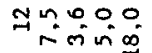 & Nom & 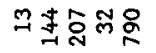 & $\therefore=00 \%$ & 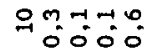 & 워ำ \\
\hline$\vec{n}$ & 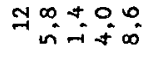 & 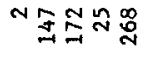 & 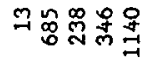 & 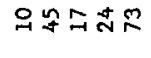 & 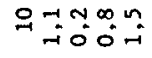 & 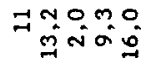 \\
\hline $\mathscr{q}$ & 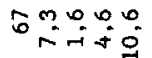 & กี ํㅜㅇำ & 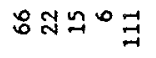 & $\mathscr{N} 9 \pm N \infty$ & \begin{tabular}{l}
$\infty 000 N$ \\
$\rightarrow 0000$ \\
\hdashline-100
\end{tabular} & 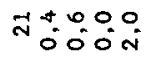 \\
\hline $\mathcal{\Psi}$ & 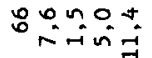 & Nㅗㅇㅛㅛ & ธี న & 축ำ용 & 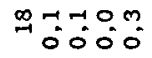 & 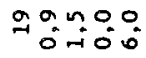 \\
\hline $\bar{N}$ & $\begin{array}{l}\infty \\
\infty \\
n \\
0\end{array}$ & INa而 & 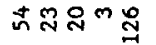 & 육ㄱㅇㅇㅇㅛ & $\begin{array}{l}30-10 \\
-100 \\
00\end{array}$ & $\because \begin{array}{l}n+1 \\
\rightarrow 00\end{array}$ \\
\hline & 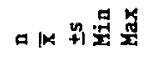 & 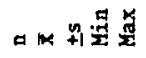 & 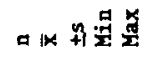 & 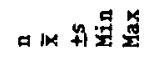 & 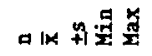 & 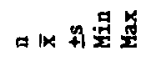 \\
\hline 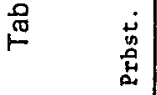 & 实 & 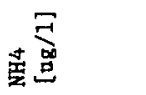 & $\underset{+\infty}{F}$ & 息 & ت己 & \&: \\
\hline
\end{tabular}




\subsubsection{Wassertemperatur}

Die Wassertemperaturmessungen weisen alle untersuchten Gewässer als sommerkühle Bergbäche mit geringen von Höhenlage und Beschattung abhängigen Unterschieden zwischen den einzelnen Probestellen aus (Tab. 11, Abb. 33). Abgesehen von $L 1$ und $L 2$ mit Temperatur-Minima von 1,5 bzw. $2,6{ }^{\circ} \mathrm{C}$ aufgrund des Zustroms von Grundwasser wurden an allen Stellen Niedrigsttemperaturen von $0^{\circ} \mathrm{C}$ registriert. In den strengen Wintern $1986 / 87$ und $1987 / 88$ waren alle Bäche teilweise zugefroren und mit Schnee bedeckt. Den weitaus größten Teil des Jahres liegen die Temperaturen unter $10^{\circ} \mathrm{C}$. Temperaturen im Maximum-Bereich, die nur an S3, M, SC und R3 $15^{\circ} \mathrm{C}$ überschreiten, treten nur an einzelnen heißen Sommertagen auf. Die an R3 1987 und 1988 gemessenen $19{ }^{\circ} \mathrm{C}$ liegen dabei deutlich über den sonst nur einige zehntel Grad über $15{ }^{\circ} \mathrm{C}$ liegenden maximalen Temperaturen der anderen Probestellen. Aus den einzelnen Meßwerten ist aber ersichtlich, daß die Monatsmitteltemperaturen auch in den Sommermonaten an allen Probestellen deutlich unter $15^{\circ} \mathrm{C}$ bleiben.

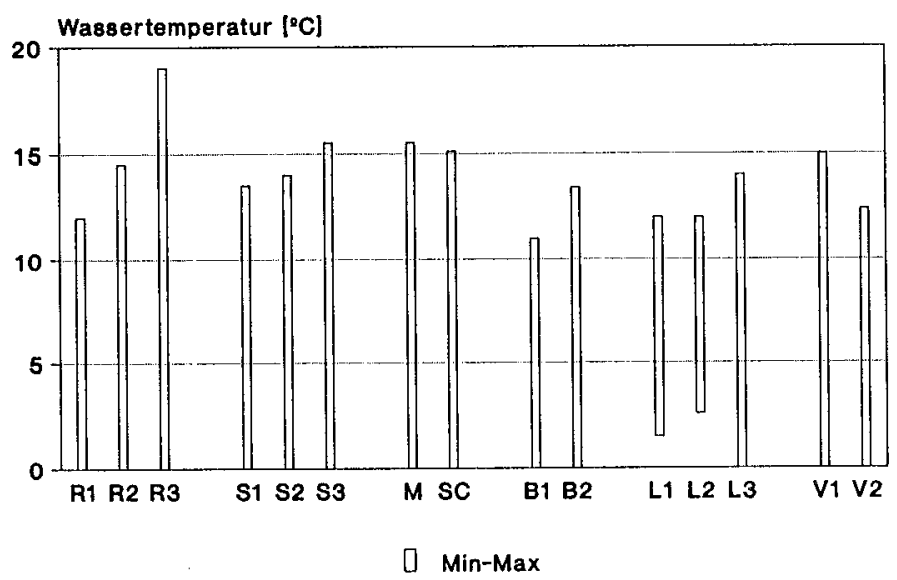

Abb. 33: Die an den Probestellen gemessenen niedrigsten und höchsten Wassertemperaturen (an R1 - R3, S1 - S3, B1, L1 und V1 auf der Grundlage von Messungen mit Min-Max-Thermometern) 


\subsubsection{Sauerstoff}

Eng mit den Wassertemperaturen verknüpft ist in nicht durch organische Schadstoffe oder andere sauerstoffzehrende Substanzen belasteten Bergbächen die Sauerstoffkonzentration, die an allen Probestellen Mittelwerte über $10 \mathrm{mg} / \mathrm{l}$ bei relativ geringen Schwankungen erreicht (Tab. 11, Abb. 34). Ein MinimumWert unter $9 \mathrm{mg} / \mathrm{l}$ wurde mit $8,6 \mathrm{mg} / \mathrm{l}$ nur an L1 gemessen, was einer Sättigung von $80 \%$ entspricht. An den restlichen Probestellen liegt die minimale Sättigung zwischen 87 und $94 \%$ (Abb. 35). Die Werte zeigen damit für die Bachfauna permanent weitestgehend optimale Sauerstoffbedingungen an allen Probestellen an.

Ergänzende $\mathrm{BSB}_{5}$-Bestimmungen erbrachten keine Hinweise auf meßbare sauerstoffzehrende Abbauprozesse.

\subsubsection{Gelöster organischer Kohlenstoff (DOC)}

Als Maß für den Huminstoffgehalt kann über den DOC der Anteil der relativ schwachen und nicht unter $\mathrm{pH}$ 3,5 - 5.0 dissoziierenden organischen Säuren an der Gewässerversauerung abgeschätzt werden (ZAHN 1990). Bei den Meßwerten heben sich B1, B2, S1 und hinsichtlich der maximalen Konzentrationen auch $M$ und SC mit erhöhten Werten von den restlichen Bächen ab (Tab. 11, Abb. 36). Mit S1, M und B1 gehören drei dieser Stellen zu den stark versauerten Probestellen. Im Einzugsgebiet der betroffenen Bäche sind vermoorte Bereiche zu finden, aus denen Huminstoffe freigesetzt werden können. Aber nur an B1 und B2 kann man anhand der Gesamtbetrachtung aller versauerungsrelevanten Anionen und Kationen von einem spürbaren Einfluß organischer Säuren auf die Versauerung sprechen (vgl. Kap. 5.1.6.2). Die im unmittelbaren Bereich eines Hangmoores liegenden Stellen L1 und L2 weisen dagegen nur niedrige DOCKonzentrationen auf. 


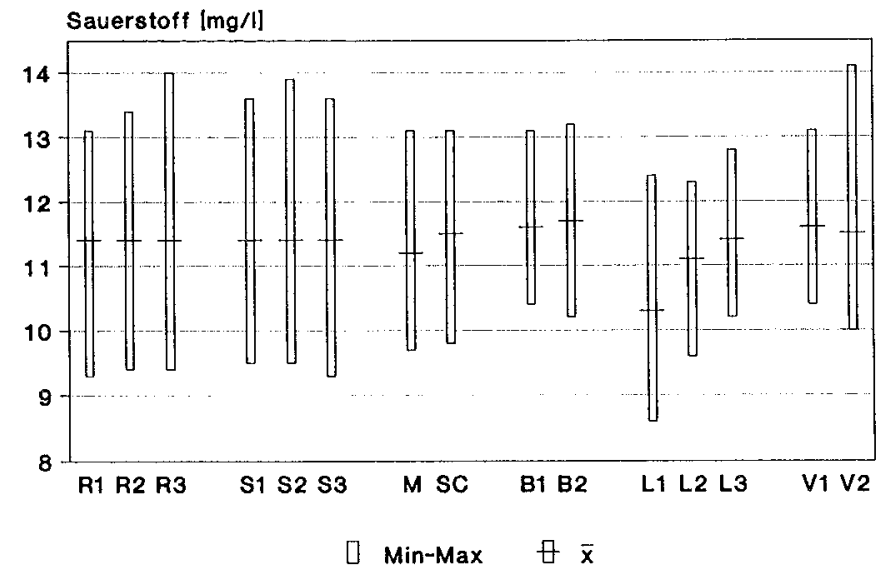

Abb. 34: Mittlere absolute Sauerstoff-Konzentration und Schwankungsbereich der Werte an den Probestellen

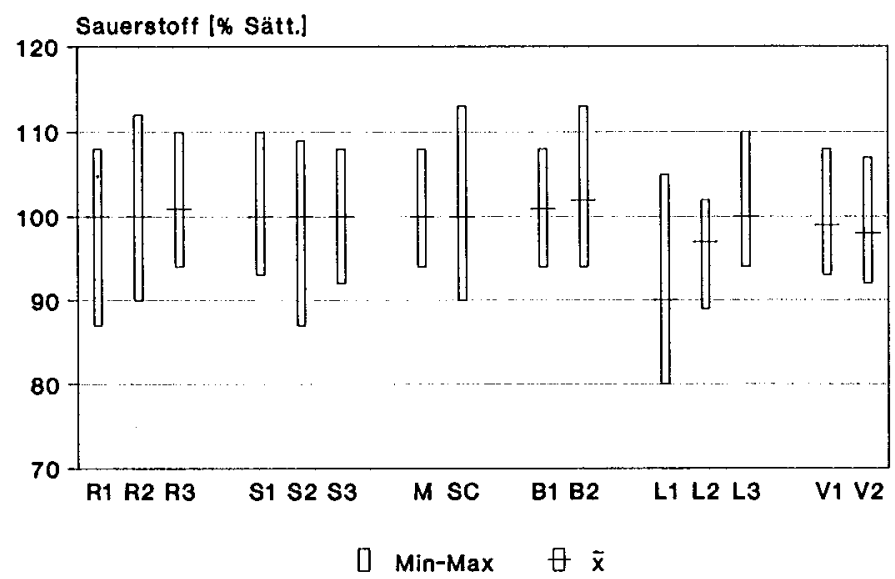

Abb. 35: Mittlere Sauerstoff-Sättigung und Schwankungsbereich der Werte an den Probestellen 


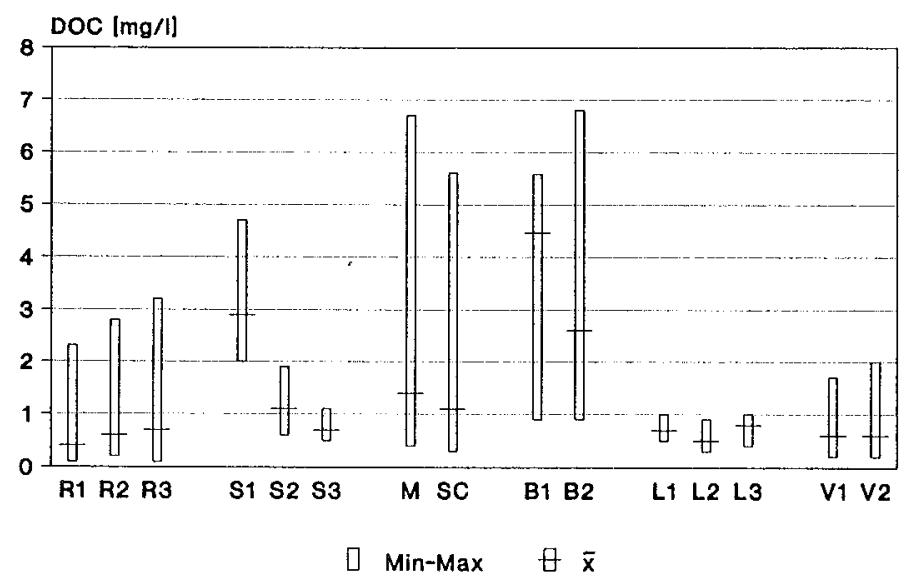

Abb. 36: Mittlere Konzentration des gelösten organischen Kohlenstoffs (DOC) und Schwankungsbereich der Werte an den Probestellen

\subsubsection{Elektrische Leitfähigkeit}

Alle untersuchten Gewässer sind mit Mittelwerten der elektrischen Leitfähigkeit $<180 \mu \mathrm{S} / \mathrm{cm}$ elektrolytarm (Tab. 11, Abb. 37). Nur die beiden nicht versauerten Bäche Alte Riefensbeek und Varleybach haben Mittelwerte $>100 \mu \mathrm{S} / \mathrm{cm}$. In der Alten Riefensbeek nimmt die Leitfähigkeit bei relativ starken Schwankungen bachabwärts von 175 auf $118 \mu \mathrm{S} / \mathrm{cm}$ ab, wohingegen sie im Varleybach von $\mathrm{V} 1$ nach V2 um fast $30 \mu \mathrm{S} / \mathrm{cm}$ ansteigt und wesentlich konstanter ist. Die Leitfähigkeiten der restlichen Bäche liegen mit Mittelwerten zwischen 57 und 84 $\mu \mathrm{S} / \mathrm{cm}$ relativ dicht zusammen.

Die Werte sind je nach Probestelle in unterschiedlichem Maße vom Abfluß abhängig. In Alter Riefensbeek und Varleybach kommt es durch den Verdünnungseffekt bei zunehmendem Abfluß in der Regel zu einem Absinken der Leitfähigkeiten. Dies ist z.B. auch an S3 zu beobachten (Abb. 38, 39). Die höchsten Meßwerte sind bei den niedrigsten Wasserständen zu registrieren. Bei gleichem Abfluß können die Unterschiede zwischen den gemessenen Leitfähigkeiten jedoch sehr groß sein. 


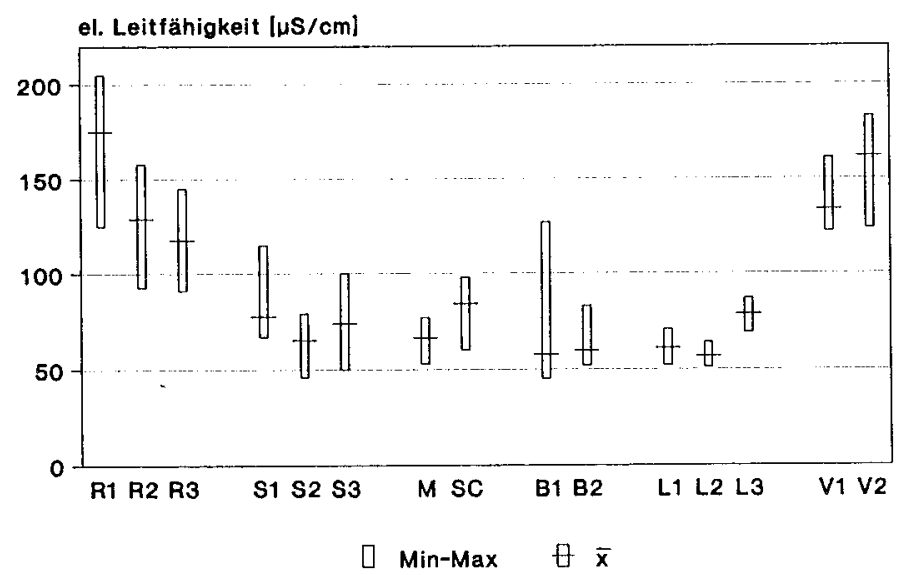

Abb. 37: Mittlere elektrische Leitfähigkeit und Schwankungsbereich der Werte an den Probestellen

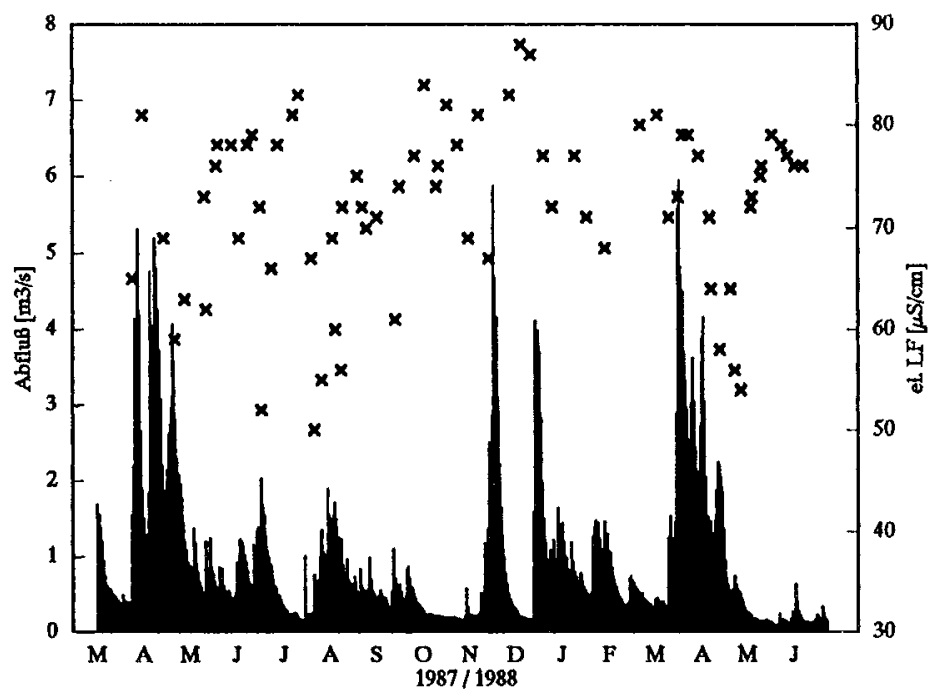

Abb. 38: Abhängigkeit der elektrischen Leitfähigkeit an $S 3(x)$ vom Abfluß am Pegel Riefensbeek (mittlere Tagesabflüsse) 


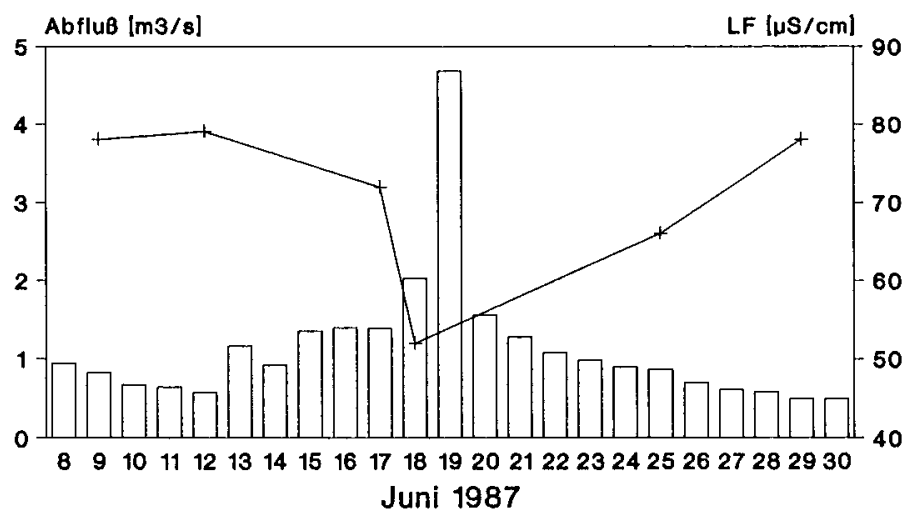

$\square$ mittl. Tagesabfluß $\quad$ el. Leitfähigkeit s3

Abb. 39: Ganglinie der Leitfähigkeit an S3 während eines am Pegel Riefensbeek registrierten Hochwassers (mittlere Tagesabflüsse)

\subsubsection{Säurekapazität und Calcium}

Als Maß für das Pufferungsvermögen steht die Säurekapazität mit dem wichtigsten Härtebildner und basischen Kation Calcium in enger Beziehung zum $\mathrm{pH}$ Wert. Aus einem Vergleich mit den $\mathrm{pH}$-Werten wird deutlich, daß bei Unterschreiten einer Säurekapazität von $0,1 \mathrm{mmol} / \mathrm{l}$ mit stärkeren $\mathrm{pH}$-Einbrüchen gerechnet werden muß. Nur an R1 erreicht die Säurekapazität einen Mittelwert $>1 \mathrm{mmol} / \mathrm{l}$ (Tab. 11, Abb. 40). An dieser Stelle wird auch der höchste gemessene Mittelwert der Calcium-Konzentration von $29,6 \mathrm{mg} / \mathrm{l}$ erreicht, die jedoch relativ großen Schwankungen unterliegt (Tab. 11, Abb. 41). Geogen bedingt nehmen beide Werte in der Alten Riefensbeek bachabwärts stark $a b$, so daß an R3 die mittlere Calcium-Konzentration um über $50 \%$ unter dem Wert von R1 liegt und auch die Säurekapazität nur noch $0,44 \mathrm{mmol} / \mathrm{l}$ erreicht. Im neutralen Varleybach ergeben sich mit 0,23 und $0,53 \mathrm{mmol} / \mathrm{l}$ deutliche Unterschiede zwischen V1 und V2. Abgesehen von L3, von der allerdings nur relativ wenige Meßwerte vorliegen, beträgt die minimale Säurekapazität an allen anderen Stellen weniger als die als kritisch anzusehenden $0,1 \mathrm{mmol} / \mathrm{l}$. Hierin zeigt sich die große Gefährdung aller dieser Probestellen gegenüber Säureschüben. Entsprechend ergeben sich für die Calcium-Konzentrationen ebenfalls relativ 
dicht beieinander liegende Werte für die schwach bis stark versauerten Stellen zwischen im Mittel 2,0 und 6,6 mg/l (S1 bzw. L3).

An S1 und B1 erreicht Natrium mit im Mittel 2,8 bzw. 3,1 mg/l bereits höhere Konzentrationen als Calcium. An B2 ist die Natrium-Konzentration fast genauso hoch wie die Calcium-Konzentration (HWW 1990).

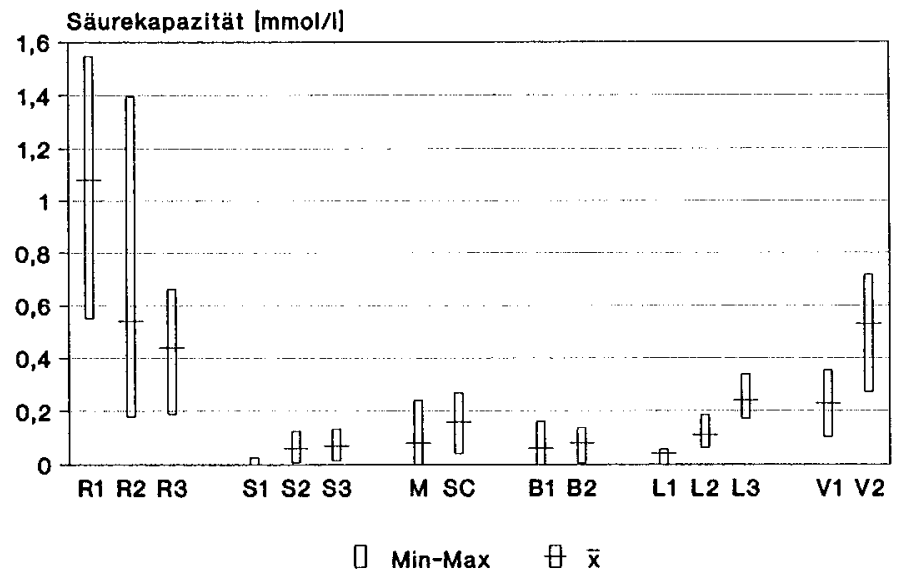

Abb. 40: Mittlere Säurekapazität und Schwankungsbereich der Werte an den Probestellen

\subsubsection{Magnesium}

Neben Calcium stellt Magnesium ein zweites wichtiges basisches Kation dar. Die Analysenwerte zeigen für dieses Element lediglich geogen bedingte geringe Unterschiede zwischen den einzelnen Gewässern mit den höchsten Mittelwerten im Varleybach $(4,0$ bzw. $4,8 \mathrm{mg} / \mathrm{l})$ und den niedrigsten in den Bode-Bächen (< $1,0 \mathrm{mg} / \mathrm{ll}$. In der Alten Riefensbeek liegt nur R1 mit 4,3 mg/l über den Werten der Probestellen der versauerten Gewässer (Tab. 11, Abb. 42). 


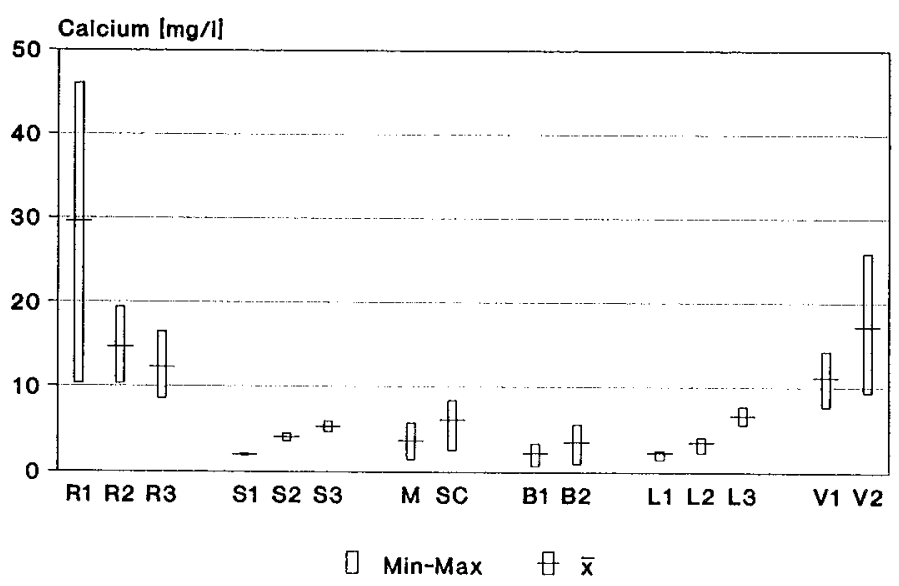

Abb. 41: Mittlere Calcium-Konzentration und Schwankungsbereich der Werte an den Probestellen

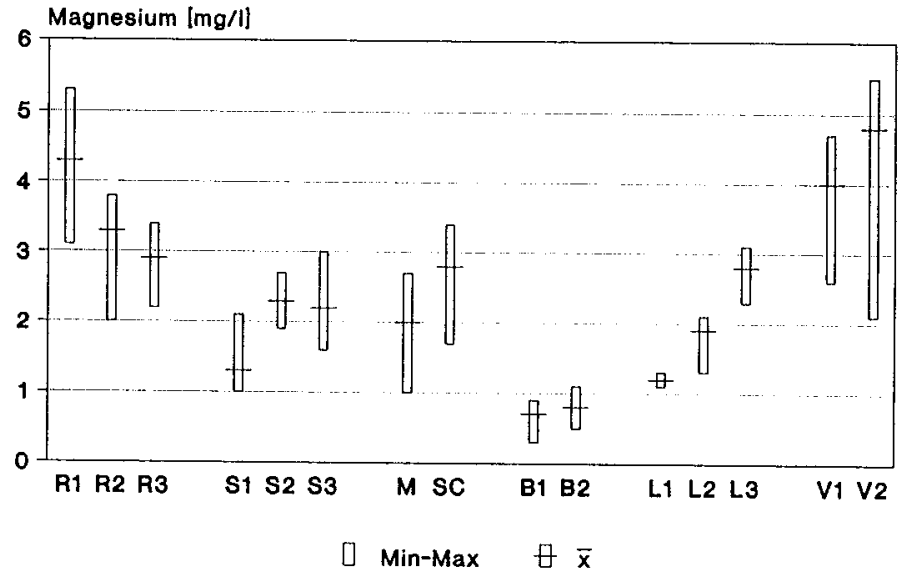

Abb. 42: Mittlere Magnesium-Konzentration und Schwankungsbereich der Werte an den Probestellen 


\subsubsection{Sulfat und Nitrat}

Sulfat und Nitrat gelten als die beiden Hauptsäurebildner der immissionsbedingten Gewässerversauerung (LENHART \& STEINBERG 1984a). Trotz der hohen Säurebelastung einiger der untersuchten Bäche ergeben sich aus den Sulfatanalysen keine Hinweise auf eine erhöhte Sulfatkonzentration in den versauerten Gewässern im Vergleich mit den nicht versauerten Bächen (Tab. 11). Die höchsten Meßwerte mit im Mittel über $30 \mathrm{mg} / \mathrm{l}$ und maximal über $50 \mathrm{mg} / \mathrm{l}$ stammen aus dem Varleybach, wohingegen sich die Mittelwerte der restlichen Probestellen alle zwischen 10 und $20 \mathrm{mg} / \mathrm{l}$ mit der Alten Riefensbeek an der oberen und den Bode-Bächen an der unteren Grenze bewegen (Abb. 43). Liegt auch keine Beziehung der Sulfat-Konzentrationen zur Gewässerversauerungssituation vor, so scheint ein Zusammenhang zur atmosphärischen Deposition jedoch zu bestehen, wie die Ergebnisse der Depositionsmessungen zeigen (vgl. Kap. 5.1.2.2). Danach sind auch die Einzugsgebiete der nicht versauerten Bäche einem hohen Luftschadstoffeintrag ausgesetzt.

Beim Sulfataustrag in die Fließgewässer spielt die sehr unterschiedliche Adsorptionskapazität der Böden eine große Rolle, die abhängt von der Bodenmächtigkeit, dem Eisen- und Aluminiumhydroxidgehalt sowie biochemischen Reaktionen (BRAHMER \& FEGER 1989). Wie hoch die Sulfat-Belastungen der Harzbäche sind, macht deutlich, daß in Wässern aus metamorphen und magmatischen Gesteinen nur natürliche Sulfat-Konzentrationen $<5$ bis $<2 \mathrm{mg} / \mathrm{l}$ zu erwarten sind (MATTHES 1963, APEL \& FAUTH 1977). In ebenfalls von der Gewässerversauerung betroffenen Gebieten des Bayerischen Waldes werden zum größten Teil nur Sulfat-Konzentrationen < $10 \mathrm{mg} / \mathrm{l}$ gemessen (SCHÖLL 1987, BAUER et al. 1988).

Die hohen Stickstoff-Depositionen spiegeln sich auch in den Gewässern wider. Die Nitrat-Konzentrationen verhalten sich bei ebenfalls geringen Unterschieden zwischen den Gewässern wie die Sulfat-Werte. Die höchsten Werte wurden mit im Mittel 10,5 und 9,4 mg/l im Varleybach gemessen (Tab. 11, Abb. 44). Es kann vermutet werden, daß die Böden mit Stickstoff gesättigt sind, und der $\mathrm{N}$ Eintrag zum größten Teil in die Gewässer gelangt. Im Frühjahr konnten mehrfach $\mathrm{pH}$-Absenkungen verbunden mit Nitratspitzen beobachtet werden, so daß das Nitrat bei ganzjährig durch hohe Sulfatkonzentrationen gesenkter Säurekapazität eine wichtige Rolle spielt (ZAHN 1990). 


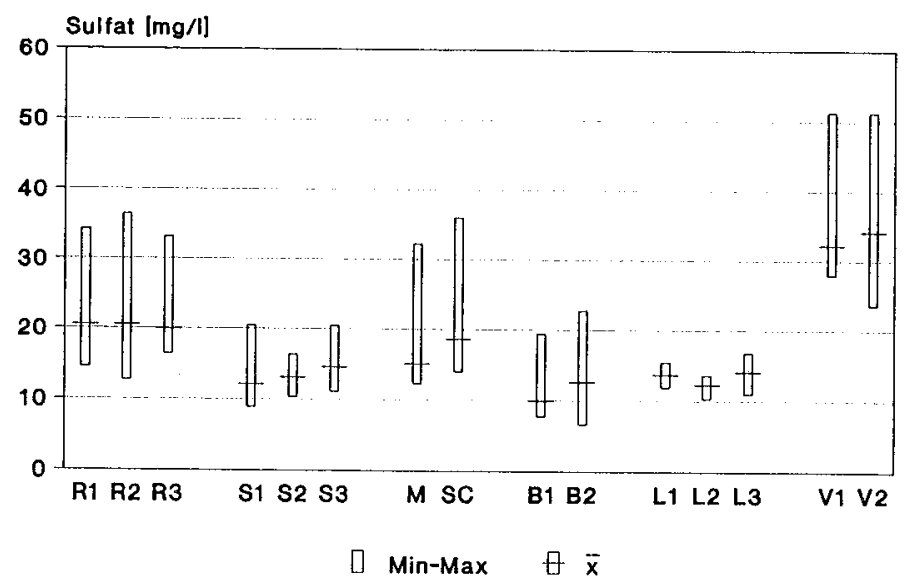

Abb. 43: Mittlere Sulfat-Konzentration und Schwankungsbereich der Werte an den Probestellen

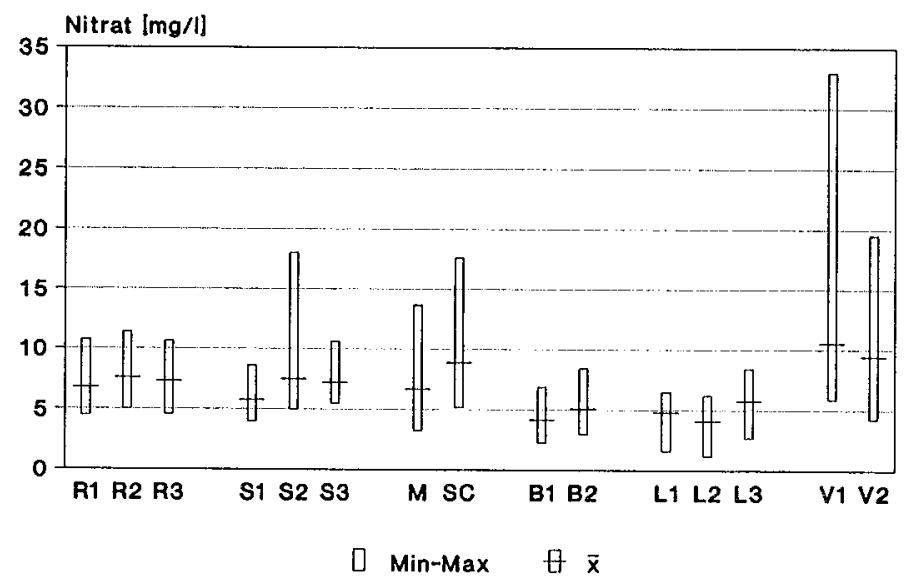

Abb. 44: Mittlere Nitrat-Konzentration und Schwankungsbereich der Werte an den Probestellen 


\subsubsection{Ammonium}

Erhöhte Ammoniumkonzentrationen können auf Belastungen durch organische Abwässer hinweisen (vgl. Kap. 5.1.5.3). Abgesehen von R2 und S1 treten keine Konzentrationen über $100 \mu \mathrm{g} / \mathrm{l}$ auf (Tab. 11, Abb. 45). Auch die beiden höheren Meßwerte liegen noch im Bereich reinen Oberflächenwassers (HÖLL 1986).

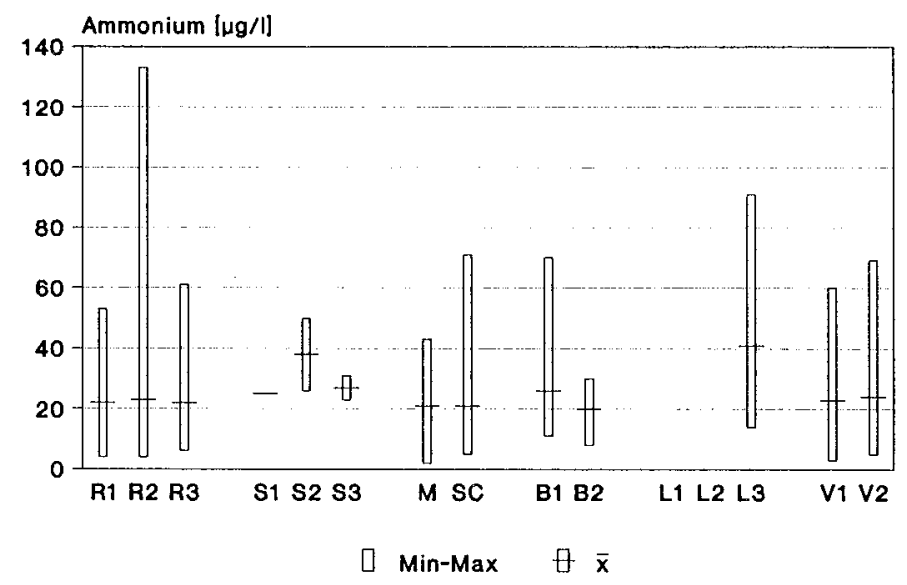

Abb. 45: Mittlere Ammonium-Konzentration und Schwankungsbereich der Werte an den Probestellen

\subsubsection{Aluminium}

Der Aluminium-Konzentration kommt im Hinblick auf die biotischen Auswirkungen der Gewässerversauerung eine wichtige Rolle zu. Aluminium kann als monomeres anorganisches $\mathrm{Al}$ (labiles $\mathrm{Al}$; $\mathrm{Al}^{3+}$, Komplexe mit $\mathrm{OH}^{-}, \mathrm{F}^{-}, \mathrm{SO}_{4}{ }^{2-}$ ), monomeres organisches $\mathrm{Al}$ (nicht-labiles $\mathrm{Al}$; Komplexe mit organischen Liganden) und colloidales oder partikuläres Al (säurelösliches Al; adsorbiertes Al, polymere Hydroxokomplexe, Al-oxide, Al-silikate) in Gewässern auftreten, über deren Verhältnis zueinander $\mathrm{pH}$-Wert, $\mathrm{Al}$ - und Huminstoff-Konzentrationen entscheiden. Die verschiedenen Species besitzen eine unterschiedliche Toxizität. Als toxisch gelten insbesondere die monomeren anorganischen Al-Species. Bei $\mathrm{pH}$-Werten $<4,5$ ist vor allem $\mathrm{Al}^{3+}$ anzutreffen, dessen Konzentrationen mit 


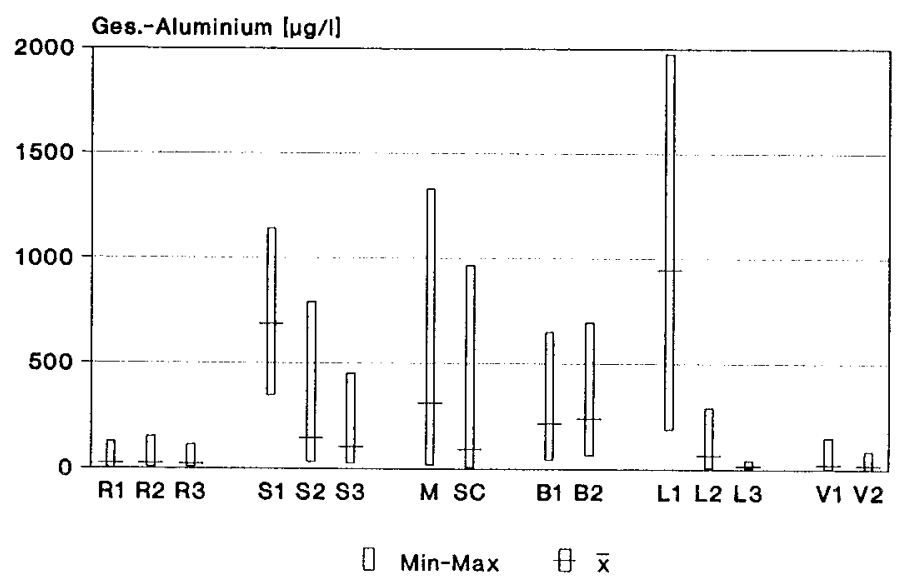

Abb. 46: Mittlere Konzentration des Gesamt-Aluminiums und Schwankungsbereich der Werte an den Probestellen

weiter abnehmenden $\mathrm{pH}-$ Werten exponentiell ansteigen. Zwischen $\mathrm{pH} \mathrm{4,5}$ und 6,5 stellen Hydroxid-Komplexe den größten Anteil dieser Al-Fraktion. Lokal in unterschiedlichen, meist geringen Anteilen sind auch Sulfat- und FluoridKomplexe zu finden. Über pH 6,5 treten monomere anorganische Al-Species als Aluminat-lonen $\mathrm{Al}(\mathrm{OH})_{4}$ auf (DRISCOLL et al. 1980, DRISCOLL 1985, TIPPING \& BACKES 1987, LAWRENCE et al. 1986).

Unter diesen verschiedenen Species kommt den Al-Hydroxid-Komplexen im Hinblick auf die biotischen Auswirkungen der Gewässerversauerung die größte Bedeutung $\mathrm{zu}$, da sie bereits in toxischen Konzentrationen in $\mathrm{pH}$-Bereichen auftreten können, in denen die Protonenkonzentration allein noch keine toxische Wirkung hat. So sind mehr als $100-200 \mu \mathrm{g} / \mathrm{l}$ monomeren anorganischen Aluminiums bei niedrigen Calcium-Konzentrationen zwischen 1 und $3 \mathrm{mg} / \mathrm{l}$ toxisch für Fische (BAKER \& SCHOFIELD 1982).

Nach den Ergebnissen der Bestimmung des Gesamt-Aluminiums lassen sich die Probestellen in zwei Gruppen teilen (Tab. 11, Abb. 46). In der ersten Gruppe sind abgesehen von $L 2$ und $L 3$ alle versauerten Probestellen zu finden, die mittlere Konzentrationen um oder bis weit über $100 \mu \mathrm{g} / 1$ und Maximum-Werte von 


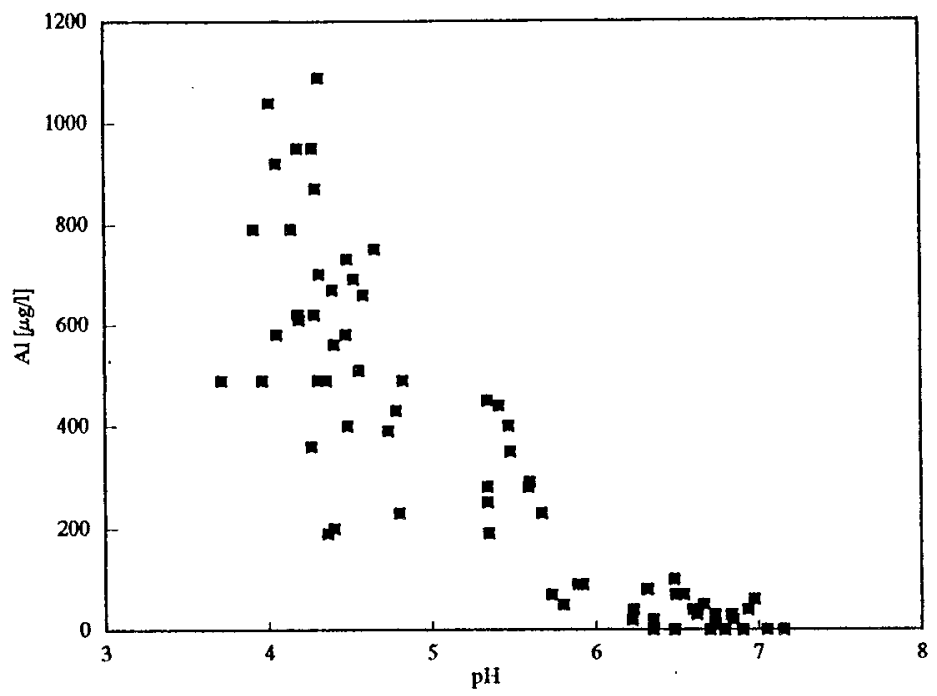

Abb. 47: Abhängigkeit der Gesamt-Aluminiumkonzentrationen vom pH-Wert

mehreren hundert $\mu \mathrm{g} / \mathrm{l}$ aufweisen. Zur zweiten Gruppe, die sich aus allen Probestellen von Alter Riefensbeek und Varleybach sowie L2 und L3 zusammensetzt, gehören die Stellen, die im Mittel deutlich unter $100 \mu \mathrm{g} / \mathrm{l}$ liegen. Aber auch hier können die gemessenen Maxima, abgesehen von L3 und V2, z.T. Werte weit über $100 \mu \mathrm{g} / \mathrm{l}$ erreichen. Im Vergleich dazu sind die Konzentrationen in Großer Söse, an M, SC, B1, B2 und an L1 bei allerdings sehr starken Schwankungen wesentlich höher. Maxima über $1000 \mu \mathrm{g} / \mathrm{l}$ werden an den stark versauerten Stellen L1 (1975 $\mu \mathrm{g} / \mathrm{l}), \mathrm{M}$ und $\mathrm{S} 1$ gemessen. L1 und S1 weisen auch die höchsten Mittelwerte auf. Ein sehr hoher Maximum-Wert konnte auch an der zu den nur schwach versauerten Probestellen gezählten Stelle SC mit $966 \mu \mathrm{g} / \mathrm{l}$ festgestellt werden.

Durch eine Auftragung der Al-Konzentrationen gegen die pH-Werte wird der exponentielle Anstieg des in den Gewässern gelösten Aluminiums unter pH 6 sehr deutlich (Abb. 47).

Wasserproben aller Probestellen außer den Stellen der Langen Bramke wurden darüber hinaus einer Speziierungsanalyse unterzogen, um die Höhe der orga- 
Tab.12: Konzentrationen des monomeren anorganischen Aluminiums an den Gesamt-Aluminiumkonzentrationen mit Mittelwerten $(\bar{x})$, Standardabweichung $( \pm s)$, Minimum und Maximum sowie Anteile der vorliegenden Bindungsformen

\begin{tabular}{|c|c|c|c|c|c|c|c|c|c|}
\hline Prbst. & & $\begin{array}{l}\text { Al ges. } \\
\text { [ug/1] }\end{array}$ & A1 anorg. & $\begin{array}{r}\text { org. } \\
x\end{array}$ & $\underset{\%}{A 1}$ ion & $\underset{\%}{\mathrm{AlOH}}$ & $\underset{\%}{\mathrm{AlOH} 2}$ & $\begin{array}{c}\text { A1OHB } \\
\%\end{array}$ & $\underset{\%}{\operatorname{ArOH}_{4}}$ \\
\hline \multirow[t]{4}{*}{ B1 } & $\overline{\mathbf{x}}$ & 215,6 & 123,3 & 66,1 & 18,9 & 5,7 & 30,3 & 43,6 & 1,3 \\
\hline & \pm 5 & 174,2 & 91,9 & 22,3 & 29,3 & 6,7 & 18,1 & 31,3 & 2,8 \\
\hline & Min & 40,0 & 30,0 & 31,4 & 0,0 & 0,0 & 4,7 & 0,8 & 0,0 \\
\hline & Max & 490,0 & 280,0 & 100,0 & 66,8 & 16,5 & 58,9 & 87,2 & 8,1 \\
\hline \multirow[t]{4}{*}{ B2 } & $\overline{\mathbf{x}}$ & 294,0 & 217,5 & 70,2 & 23,6 & 5,9 & 23,8 & 45,5 & 1,3 \\
\hline & $\pm s$ & 251,1 & 199,9 & 24,3 & 33,6 & 6,5 & 19,8 & 37,7 & 1,4 \\
\hline & Min & 20,0 & 15,0 & 28,6 & 0,0 & 0,0 & 4,9 & 0,1 & 0,0 \\
\hline & Max & 700,0 & 530,0 & 100,0 & 85,4 & 17,3 & 63,2 & 86,5 & 4,0 \\
\hline \multirow[t]{4}{*}{ M } & $\overline{\mathbf{x}}$ & 593,3 & 580,0 & 98,2 & 40,6 & 15,6 & 35,8 & 7,9 & 0,0 \\
\hline & \pm 8 & 298,9 & 287,9 & 3,6 & 20,1 & 5,1 & 13,3 & 12,1 & 0,0 \\
\hline & Min & 0,0 & 0,0 & 89,3 & 1,4 & 4,3 & 19,7 & 1,0 & 0,0 \\
\hline & $\operatorname{Max}$ & 1090,0 & 1050,0 & 100,0 & 62,2 & 18,5 & 59,3 & 34,9 & 0,1 \\
\hline \multirow[t]{4}{*}{$\mathrm{S} 1$} & $\overline{\mathbf{x}}$ & 713,6 & 682,9 & 95,7 & 74,6 & 13,5 & 11,5 & 0,5 & 0,0 \\
\hline & $\pm s$ & 184,5 & 181,4 & 4,0 & 6,6 & 2,1 & 4,3 & 0,3 & 0,0 \\
\hline & Min & 490,0 & 450,0 & 87,3 & 64,1 & 8,8 & 4,0 & 0,1 & 0,0 \\
\hline & Max & 1040,0 & 1030,0 & 100,0 & 87,1 & 16,4 & 18,6 & 0,9 & 0,0 \\
\hline \multirow[t]{4}{*}{$\mathrm{S} 2$} & $\overline{\mathbf{x}}$ & 246,7 & 193,4 & 71,9 & 11,4 & 6,2 & 30,5 & 50,2 & 1,7 \\
\hline & \pm 8 & 237,6 & 199,7 & 28,9 & 18,6 & 7,5 & 20,4 & 35,4 & 2,0 \\
\hline & Min & 0,0 & 0,0 & 17,5 & 0,0 & 0,0 & 6,3 & 1,4 & 0,0 \\
\hline & Max & 690,0 & 580,0 & 100,0 & 57,8 & 17,6 & 63,4 & 87,6 & 6,1 \\
\hline \multirow[t]{4}{*}{ S3 } & $\overline{\mathbf{x}}$ & 145,5 & 114,7 & 69,3 & 0,7 & 1,9 & 30,9 & 64,2 & 2,4 \\
\hline & $\pm \mathbf{s}$ & 163,4 & 141,6 & 31,1 & 1,0 & 2,4 & 23,4 & 24,3 & 2,3 \\
\hline & Min & 0,0 & 0,0 & 11,1 & 0,0 & 0,0 & 6,5 & 27,6 & 0,1 \\
\hline & Max & 450,0 & 390,0 & 100,0 & 2,7 & 6,3 & 63,4 & 87,6 & 5,9 \\
\hline \multirow[t]{4}{*}{ SC } & $\overrightarrow{\mathbf{x}}$ & 90,0 & 65,7 & 69,0 & 0,2 & 1,0 & 25,9 & 69,7 & 3,1 \\
\hline & $\pm \mathbf{6}$ & 107,7 & 96,0 & 31,8 & 0,3 & 1,3 & 20,4 & 19,5 & 3,1 \\
\hline & Min & 0,0 & 0,0 & 22,2 & 0,0 & 0,0 & 5,1 & 42,5 & 0,2 \\
\hline & $\operatorname{Max}$ & 290,0 & 280,0 & 100,0 & 0,7 & 2,9 & 53,7 & 87,4 & 7,4 \\
\hline
\end{tabular}

nisch und anorganisch gebundenen Konzentrationsanteile zu erhalten (Tab. 12). In Varleybach und Alter Riefensbeek liegen die Konzentrationen unterhalb der Nachweisgrenze $(10 \mu \mathrm{g} / \mathrm{l})$ der hierbei angewandten Methodik. Für die restlichen Gewässer ergibt sich im Mittel ein anorganisch gebundener Anteil zwischen 66 $\%$ an $\mathrm{B} 1$ bis zu $98 \%$ an M. Für S2, S3, SC und B2 errechnen sich Mittelwerte von rund $70 \%$, für $S 1$ von $96 \%$. 
Insbesondere für die Zeiten niedriger $\mathrm{pH}$-Werte mit sehr hohen Konzentrationen an Gesamt-Aluminium zeigt sich an B1 und in abgeschwächter Form auch an B2 aufgrund der hohen Huminstoffgehaite, wie sie durch den DOC angezeigt werden, eine Verminderung der anorganischen Al-Konzentrationen im Vergleich zu S1 und $M$ mit nur sehr geringen Anteilen organisch gebundenen Aluminiums. Bei $\mathrm{pH}$-Werten < 5 steigen aber auch an B1 die anorganischen Al-Spezies auf Konzentrationen weit über $100 \mu \mathrm{g} / \mathrm{l}$ an.

Mit dem Auftreten insbesondere fischtoxischer Konzentrationen monomeren anorganischen Aluminiums mit einem hohen Anteil an Al-Hydroxiden ist zumindest zeitweilig an B1, B2, M, S1, S2, S3 und SC zu rechnen. Da die DOCGehalte an L1 sehr niedrig sind, muß auch L.1 in die Aufzählung mit einbezogen werden, ohne daß von dieser Stelle eine Speziierungsanalyse vorliegt.

\subsubsection{Zink, Cadmium, Blei}

Von den wichtigsten durch die atmosphärische Deposition in die Ökosysteme eingetragenen und durch die Versauerung mobilisierten Schwermetallen wurden die Konzentrationen von Zink, Cadmium und Blei bestimmt.

Die niedrigsten Zink-Konzentrationen treten in der Alten Riefensbeek, die höchsten in der Langen Bramke (bis $116 \mu \mathrm{g} / 1$ ) auf (Tab. 11, Abb. 48). Der Varleybach weist ähnliche Werte wie die versauerten Gewässer auf, was wiederum die hohe Belastung auch dieses Einzugsgebiets unterstreicht. In den versauerten Bächen ist bachabwärts entsprechend den $\mathrm{pH}$-Gradienten der Trend einer Konzentrationsabnahme zu erkennen. Die Meßwerte von L1 liegen über dem EG-Trinkwassergrenzwert von $100 \mu \mathrm{g} / \mathrm{l}$ (HÖLL 1986). Der natürliche Konzentrationsbereich in Gewässern von bis zu $10 \mu \mathrm{g} / /$ (DFG 1982) wird an S1, M, SC, L2 und auch im Varleybach deutlich überschritten.

Cadmium ist nach Blei das zweithäufigste Schwermetall in der Luft, das vor allem durch die Verbrennung fossiler Brennstoffe in die Atmosphäre gelangt (UBA 1977). Bei ihm ergeben sich direkte Beziehungen zu den pH-Verhältnissen. Sehr niedrige Cadmium-Konzentrationen, überwiegend unterhalb der Nachweisgrenze, sind in den nicht versauerten Gewässern Varleybach und Alte Riefensbeek zu verzeichnen (Tab. 11, Abb. 49). Die höchsten Mittelwerte haben die stark versauerten Probestellen L1, S1 und $M$, wobei die Werte an L1 und S1 wie 
die $\mathrm{pH}-$ Werte auch sehr konstant sind und an $\mathrm{M}$ entsprechend den $\mathrm{pH}-$ Werten großen Schwankungen unterliegen. Die stärker von organischen Säuren beeinflußten Stellen B1 und B2 haben einen relativ niedrigen Mittelwert mit größeren Schwankungen. Die mäßig und schwach versauerten Gewässer weisen ebenfalls deutlich höhere Konzentrationen auf als die nicht versauerten Bäche. Alle Cadmium-Werte liegen zwar unterhalb des EG-Trinkwassergrenzwertes von $\mathbf{5}$ $\mu \mathrm{g} / /$ (HÖLL 1986), die Werte an L1, S1 und $M$ stellen dennoch für obere Bachabschnitte sehr hohe Konzentrationen dar. FAUTH et al. (1983) fanden bei flächendeckenden. Untersuchungen in $75 \%$ dieser Gewässerabschnitte nur Cadmium-Konzentrationen $<0,6 \mu \mathrm{g} / \mathrm{l}$ und bei nur $1 \%$ höhere Konzentrationen als $2,1 \mu \mathrm{g} / \mathrm{l}$.

Die höchsten Blei-Konzentrationen wurden an den stark versauerten Stellen S1 $(13,2 \mu \mathrm{g} / 1), \mathrm{L} 1, \mathrm{M}$ und B1 gemessen (Tab. 11, Abb. 50). An den mäßig und schwach versauerten Stellen ergeben sich Meßwerte im Bereich wie sie auch in der Alten Riefensbeek auftreten. Im Varleybach liegen die Meßwerte meist unterhalb der Nachweisgrenze. Die Konzentrationen an S1 und L1 bleiben stets über dem EG-Trinkwassergrenzwert von $5 \mu \mathrm{g} / \mathrm{l}$ (HÖLL 1986). Den natürlichen Konzentrationsbereich in Gewässern von bis zu 0,2 $\mu \mathrm{g} / \mathrm{l}$ (DFG 1982) überschreiten aber die Mittelwerte aller Probestellen außer SC, V1 und V2.

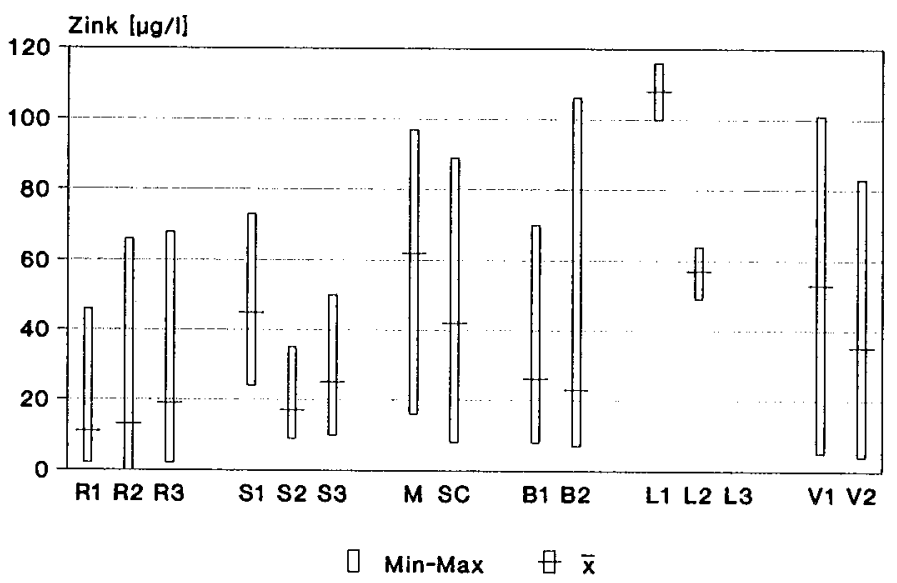

Abb. 48: Mittlere Zink-Konzentration und Schwankungsbereich der Werte an den Probestellen 


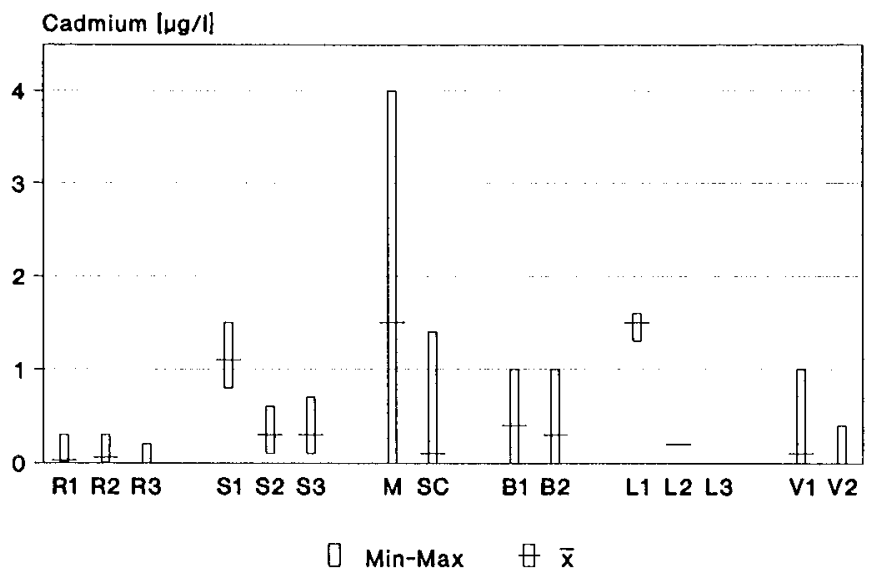

Abb. 49: Mittlere Cadmium-Konzentration und Schwankungsbereich der Werte an den Probestellen

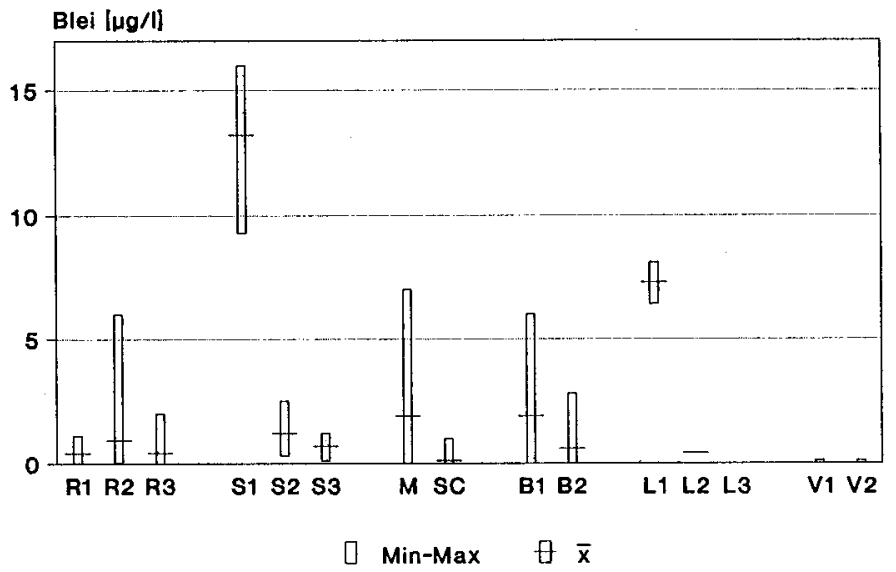

Abb. 50: Mittlere Blei-Konzentration und Schwankungsbereich der Werte an den Probestellen 
5.1.6 Zusammenfassende Betrachtung der Hydrochemie der Probestellen und der Gewässersysteme

\subsubsection{Vergleich der Probestellen}

Eine hydrochemische Abgrenzung der Probestellen kann, wie die Betrachtung der einzelnen gemessenen Parameter gezeigt hat, nicht allein über die pH-Werte erfolgen. Zwar ergibt sich bei einer Korrelationsanalyse der Gesamtdaten eine überwiegend hohe, hoch signifikante Korrelation des $\mathrm{pH}$ mit acht der zwölf Parameter (Tab. 13) (insbes. Säurekapazität, basische Kationen und Aluminium), einige Probestellen weisen jedoch geogen oder eintragsbedingte besondere Charakteristika auf, die eine nur auf den $\mathrm{pH}$ ausgerichtete Betrachtung nicht zulassen.

Ein bemerkenswertes Ergebnis der Korrelationsanalyse ist die positive Korrelation zwischen $\mathrm{pH}$ und Sulfat bei gleichzeitiger positiver Korrelation zwischen Sulfat und Nitrat. Dieses Ergebnis ist darauf zurückzuführen, daß die beiden Hauptsäurebildner in den nicht versauerten Bächen in höheren Konzentrationen auftreten als in den bereits versauerten, was sehr deutlich auf die auf den gesamten Untersuchungsraum einwirkenden hohen Schadstoffbelastungen hinweist, die aber in den Einzugsgebieten von Alter Riefensbeek und Varleybach aufgrund der geogenen und pedologischen Verhältnisse noch nicht zu einer spürbaren Gewässerversauerung geführt haben.

Weiteren Aufschluß über die an den Probestellen wirksamen Puffersysteme und eventuell auch auf die Versauerungsgefährdung kann der von SCHOEN (1985) nach HENRIKSEN (1979) modifizierte Quotient $(\mathrm{Ca}+\mathrm{Mg}) /\left(\mathrm{SO}_{4}+\mathrm{NO}_{3}\right)$ geben. Ein Verhältnis der wichtigsten basischen Kationen zu den Hauptsäurebildnern > 1,5 und damit keine akute Versauerungsgefährdung weisen allein die Probestellen der Alten Riefensbeek auf (Abb. 51). Ein über 1,0 liegender Quotient, der anzeigt, daß an den Probestellen das Hydrogenkarbonat-Puffersystem noch wirksam ist, errechnet sich für V2, L3, S3 und V1. SC liegt genau auf dem Grenzwert, und an den restlichen Stellen, an denen die mittleren $\mathrm{pH}$-Werte < 5,5 sind, erfolgt die Pufferung über Aluminium-Verbindungen oder Huminstoffe. Insbesondere V1 und auch SC müssen nach diesem Kriterium als wesentlich stärker versauerungsgefährdet angesehen werden, als dies auf der Grundlage der $\mathrm{pH}-$ Minima zu vermuten ist, und wie es sich auch in den mittleren $\mathrm{pH}$-Werten ausdrückt. Insgesamt scheint daher die Brauchbarkeit des Quotienten zur Charakterisierung der $\mathrm{pH}-$ Verhältnisse als relativ gering anzusehen zu sein. 

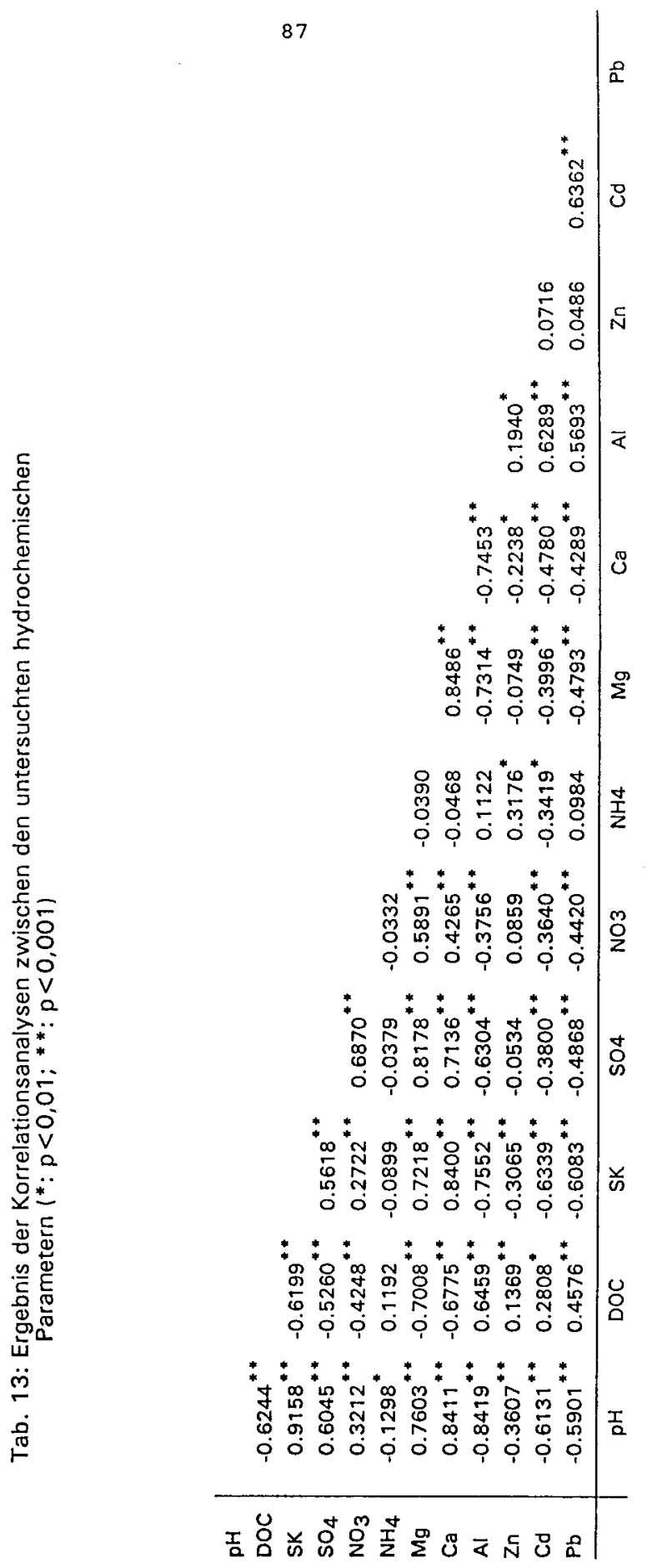


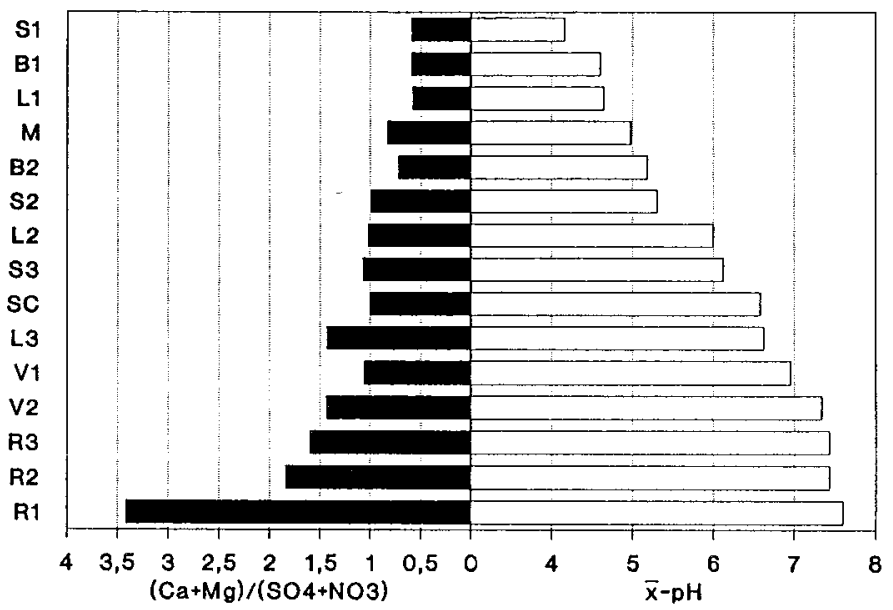

Abb. 51: Verhältnis der wichtigsten basischen Kationen zu den Hauptsäurebildnern (SCHOEN-Quotient) im Vergleich mit den mittleren $\mathrm{pH}$-Werten
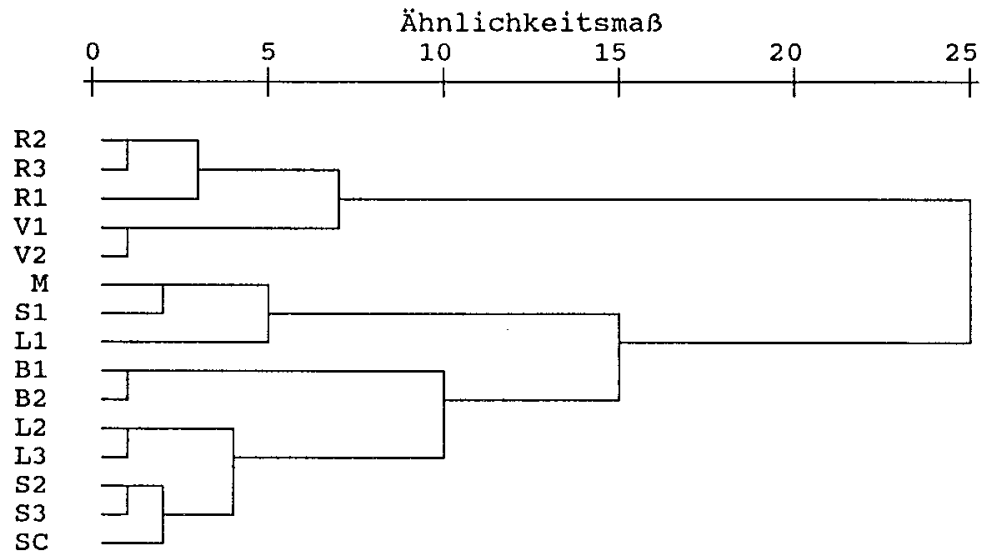

Abb. 52: Dendrogramm zur Cluster-Analyse der hydrochemischen Daten nach dem WARD-Verfahren 
Cluster-Analysen ermöglichen es, unter Einbeziehung aller gemessenen Parameter einen hydrochemischen Ähnlichkeitsvergleich der Probestellen durchzuführen. Hierzu wurde aus den Mittelwerten der einzelnen Parameter eine Datenmatrix erstellt. Nach Z-Standardisierung wurden $\mathrm{Mg}$ und $\mathrm{Ca}$ wegen einer Korrelation von $r>0,9$ mit der Leitfähigkeit bzw. mit Leitfähigkeit und Säurekapazität sowie $\mathrm{Pb}$ wegen fehlender Normalverteilung der Werte von der Analyse ausgeschlossen (vgl. BACKHAUS et al. 1989). Die Single Linkage-Analyse erbrachte keinen größeren Ausreißer. Nur L1 setzt sich aufgrund relativ hoher Al- und $\mathrm{Zn}$ Konzentrationen von den anderen Stellen etwas stärker ab. Die Cluster-Analyse nach dem WARD-Verfahren zeigt vier voneinander gut zu trennende Gruppen, wobei zwei Hauptgruppen ausgewiesen sind, von denen sich die zweite Hauptgruppe noch weiter auftrennen läßt (Abb. 52):

$\mathrm{Zu}$ allen Gruppenabgrenzungen tragen $\mathrm{pH}$ und $\mathrm{Al}$ in unterschiedlichem Ausmaß bei. Die Zahl der Parameter anhand derer die Abgrenzungen erfolgt sind, ist mit sieben zwischen den Gruppen 1 und 2 am höchsten und liegt bei den Abgrenzungen innerhalb der Gruppe 2 bei drei bis vier. Zwischen Gruppe 1 und 2 bestehen große Unterschiede bei Leitfähigkeit $(>115 \mu \mathrm{S} / \mathrm{cm}-<85 \mu \mathrm{S} / \mathrm{cm})$ und Calcium-Konzentrationen ( $>11 \mathrm{mg} / 1 \quad-<7 \mathrm{mg} /$ ), außerdem bei $\mathrm{pH}$, Säurekapazität, $\mathrm{Mg}, \mathrm{Al}$ und $\mathrm{SO}_{4}$, so daß sich in der ersten Gruppe nur die nicht versauerten Probestelien der Alten Riefensbeek und des Varleybachs wiederfinden. In der zweiten Hauptgruppe sind dagegen die versauerten Stellen zusammengefaßt. Zwischen innen zeigen sich aber auch Unterschiede, bei denen der $\mathrm{pH}$ nur in zweiter Linie zur Abgrenzung beiträgt. Gruppe 2.1 und 2.2 lassen sich aufgrund großer Unterschiede in den Al- $(>300 \mu \mathrm{g} / \mathrm{l}-<240 \mu \mathrm{g} / \mathrm{l})$ und Cadmium-Konzentrationen $(>1,0 \mu \mathrm{g} / \mathrm{l}-<0,5 \mu \mathrm{g} / \mathrm{l})$ voneinander abgrenzen, so daß hierdurch die am stärksten versauerten Probestellen in einer Gruppe stehen. Von der Gruppe 2.2.2 setzt sich die Gruppe 2.2.1 durch deutlich höhere DOC- $(>2,6 \mathrm{mg} / \mathrm{l}-<1,2 \mathrm{mg} / \mathrm{l})$ und Al- $(>210 \mu \mathrm{g} / \mathrm{l}-<150 \mu \mathrm{g} / \mathrm{l})$ sowie niedrigere $\mathrm{Mg}$-Konzentrationen (<0,9 mg/l - >1,9 mg/l) ab. Uneinheitlich über alle Gruppen gestreut und damit für die Einteilung ungeeignet sind die Konzentrationen von $\mathrm{NO}_{3}, \mathrm{Zn}$ und $\mathrm{Pb}$. 


\subsubsection{Hydrochemie der Gewässersysteme}

Die zusammenfassende Betrachtung der bearbeiteten Bachsysteme zeigt die Alte Riefensbeek als gut gepuffertes Gewässer mit stabilen pH-Werten im neutralen Bereich und einer hohen Leitfähigkeit aufgrund hoher Konzentrationen der aus der Verwitterung stammenden basischen Kationen und daraus resultierender hoher Säurekapazität. DOC-, Al- und Schwermetall-Konzentrationen sind niedrig; der $\mathrm{SO}_{4}$-Austrag ist aufgrund hoher atmosphärischer Deposition sehr hoch. Bachabwärts sinken geogen bedingt die Konzentrationen an $\mathrm{Ca}$ und $\mathrm{Mg}$ bei damit verbundener Abnahme von Leitfähigkeit und Säurekapazität.

Auch der Varleybach ist bei hohen Konzentrationen der basischen Kationen $\mathrm{Ca}$ und $\mathrm{Mg}$ ein relativ gut gepuffertes Gewässer mit $\mathrm{pH}$-Schwankungen ausschließlich im neutralen Bereich, das jedoch die höchsten Sulfat- und Nitratbelastungen aller untersuchten Gewässer aufweist. Die meisten Parameter zeigen ähnliche Verhältnisse an wie in der Alten Riefensbeek. Die minimal gemessene Säurekapazität von nur $0,104 \mathrm{mmol} / \mathrm{l}$ und der niedrige SCHOEN-Quotient von 1,06 weisen jedoch die Probestelle $\vee 1$ als an der Schwelle zur Versauerung stehend aus. Die dagegen deutlich höheren Konzentrationen der basischen Kationen an V2 zeigen für diese Stelle eine weitaus geringere Gefährdung an.

An der Großen Söse ist mit S1 die permanent am stärksten versauerte Probestelle der Untersuchungen zu finden. Relativ hohe DOC-Gehalte weisen auf eine Huminstoffbeteiligung an der Versauerung hin. Gleichzeitig sind die $\mathrm{Ca}-$ und $\mathrm{Mg}-$ Konzentrationen sehr niedrig und Al- und Schwermetall-Werte hoch. Bachabwärts nehmen $\mathrm{pH}$, Säurekapazität und basische Kationen bei sinkenden Al- und Schwermetall-Gehalten zu.

Die Probestelle am Großen Mollenbach ist mit ihren den größten Teil des Jahres sehr niedrigen pH-Werten, der überwiegend niedrigen Säurekapazität, relativ hohen DOC-, Sulfat- und Nitrat-Konzentrationen zu den stark versauerten Probestellen zu zählen. Bei Niedrigabflüssen im Sommerhalbjahr können pH-Werte im neutralen Bereich erreicht werden. Die im Vergleich zu M im Mittel höhere Säurekapazität aufgrund höherer Konzentrationen der basischen Kationen kann an SC bei den ansonsten ähnlichen hydrochemischen Verhältnissen ein Absinken des $\mathrm{pH}$ unter 5 verhindern. 
Große Bode und Warme Bode zeichnen sich durch eine von Huminstoffen mitbestimmte Versauerung aufgrund der Vermoorung großer Teile des Einzugsgebiets aus. Sulfat und Nitrat aber auch die basischen Kationen liegen im niedrigsten Bereich der im gesamten Untersuchungsprogramm gemessenen Werte. Trotz niedriger $\mathrm{pH}$-Werte sind die Konzentrationen an Aluminium und Schwermetallen relativ gering.

An der Langen Bramke weist L1 sehr hohe Aluminium- und SchwermetallKonzentrationen bei permanent niedrigen $\mathrm{pH}$-Werten auf. Der Huminstoffeinfluß ist trotz größerer Vermoorung des Quellgebiets bis hinab zu L2 gering. Die Belastungen durch Sulfat und Nitrat liegen im mittleren Bereich. Gleichzeitig ist aber im gesamten Gewässer die Konzentration der basischen Kationen relativ gering, so daß insbesondere an L2 stärkere pH-Schwankungen auftreten und auch L3 periodisch eine schwache Versauerung zeigt. 


\subsection{Artenspektrum der Gewässerbiozönosen}

An den 15 in die Untersuchungen einbezogenen Probestellen konnten insgesamt 76 Arten oder höhere Taxa der bearbeiteten Makrofauna-Gruppen Gastropoda (1 Art), Bivalvia (1 Art), Amphipoda (3 Arten), Ephemeroptera (19 Arten), Plecoptera (35 Arten), Coleoptera (15 Arten) und Pisces (2 Arten) nachgewiesen werden (Tab. 14). Nur Plecopteren kommen an allen Probestellen vor. Coleopteren fehlen an B1, Ephemeropteren an S1, B1 und L1. Amphipoden sind an 9 und Molluscen an 7 Stellen zu finden. Fische gibt es an den stark versauerten Probestellen S1, M, B1 und L1 nicht (Abb. 53).

Die höchsten Artenzahlen konnten in den beiden nicht versauerten Gewässern, in der neben der Großen Söse einer intensiveren Untersuchung unterzogenen Alten Riefensbeek mit 55 - 58 Arten und im Varleybach mit 42 - 44 Arten, festgestellt werden. Eine relativ hohe Artenzahl haben mit jeweils 39 Arten auch S3 und SC. Nur 10 der insgesamt nachgewiesenen 76 Arten kommen in der Alten Riefensbeek nicht vor. In der Großen Söse sind mit den Ephemeropteren Siphlonurus aestivalis, Baetis vernus und Paraleptophlebia submarginata sowie dem Coleopteren Hydroporus nigrita vier Arten zu finden, die in der Alten Riefensbeek nicht nachgewiesen werden konnten. 31 Arten der Alten Riefensbeek wurden dagegen in der Großen Söse nicht angetroffen. Die artenärmsten Biozönosen haben mit 9 bzw. 10 Arten L1 und B1.

Auch bei den Trichopteren zeigt sich eine große Abhängigkeit der durch Emergenzfallen und Benthosaufsammlungen nachgewiesenen Artenzahlen von der Versauerung (RÜDDENKLAU 1989). Während in den nicht versauerten Bächen mit 27 - 35 Arten in der Alten Riefensbeek und 22 - 24 Arten im Varleybach die höchsten Artenzahlen gefunden wurden, kommen an den stark versauerten Stellen nur noch 6 - 9 Trichopteren-Arten vor (Tab. 15).

Unter Berücksichtigung aller Insektenordnungen, außer den bisher noch nicht abschließend bearbeiteten Dipteren, weisen damit die Trichopteren an allen nicht versauerten Stellen die höchsten Artenzahlen auf. Es folgen Plecopteren, Ephemeropteren und Coleopteren. In den versauerten Gewässern ändert sich diese Reihenfolge an den meisten Stellen zugunsten der Plecopteren und Coleopteren, die dort höhere Artenzahlen erreichen als Trichopteren bzw. Ephemeropteren. 


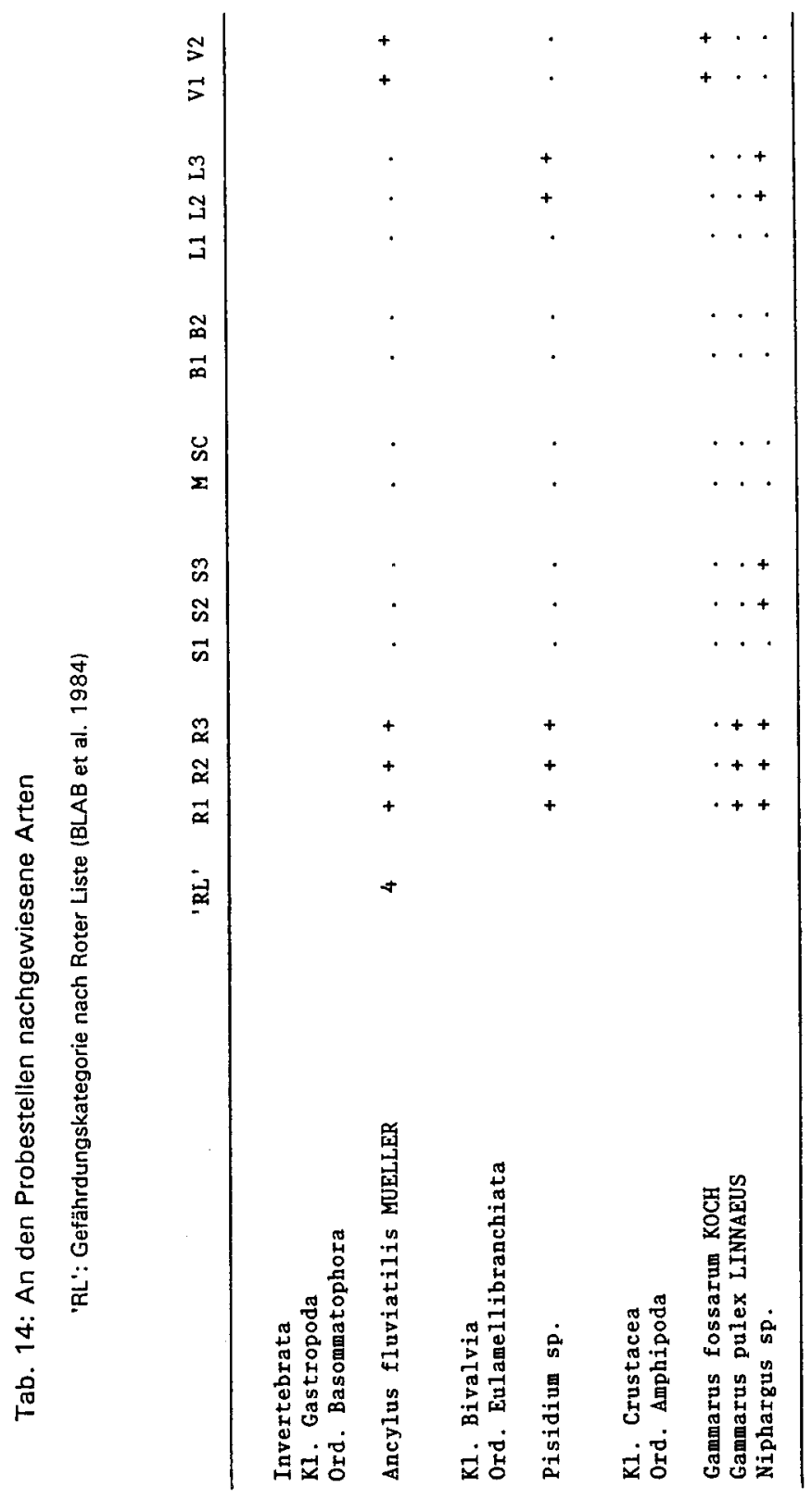




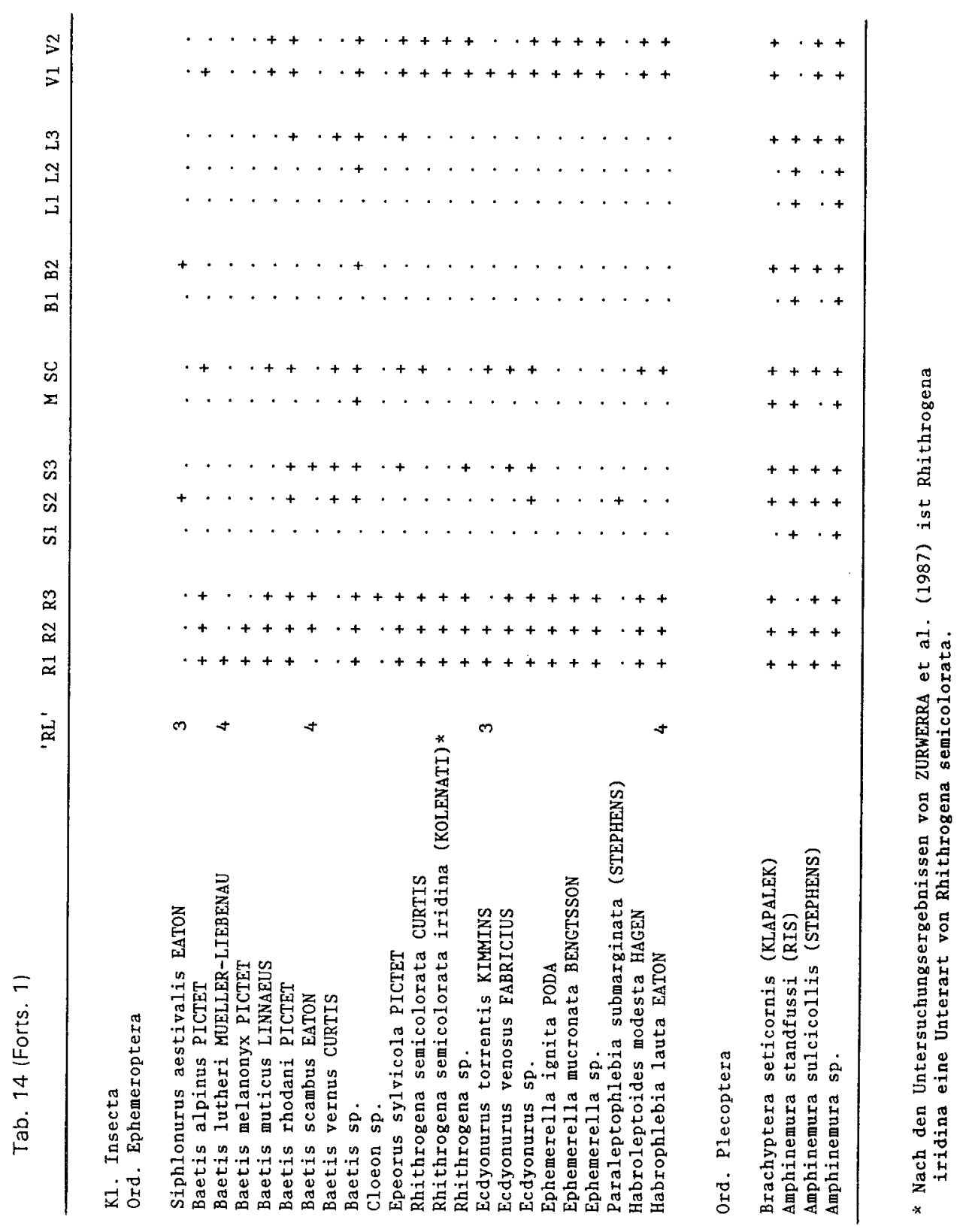




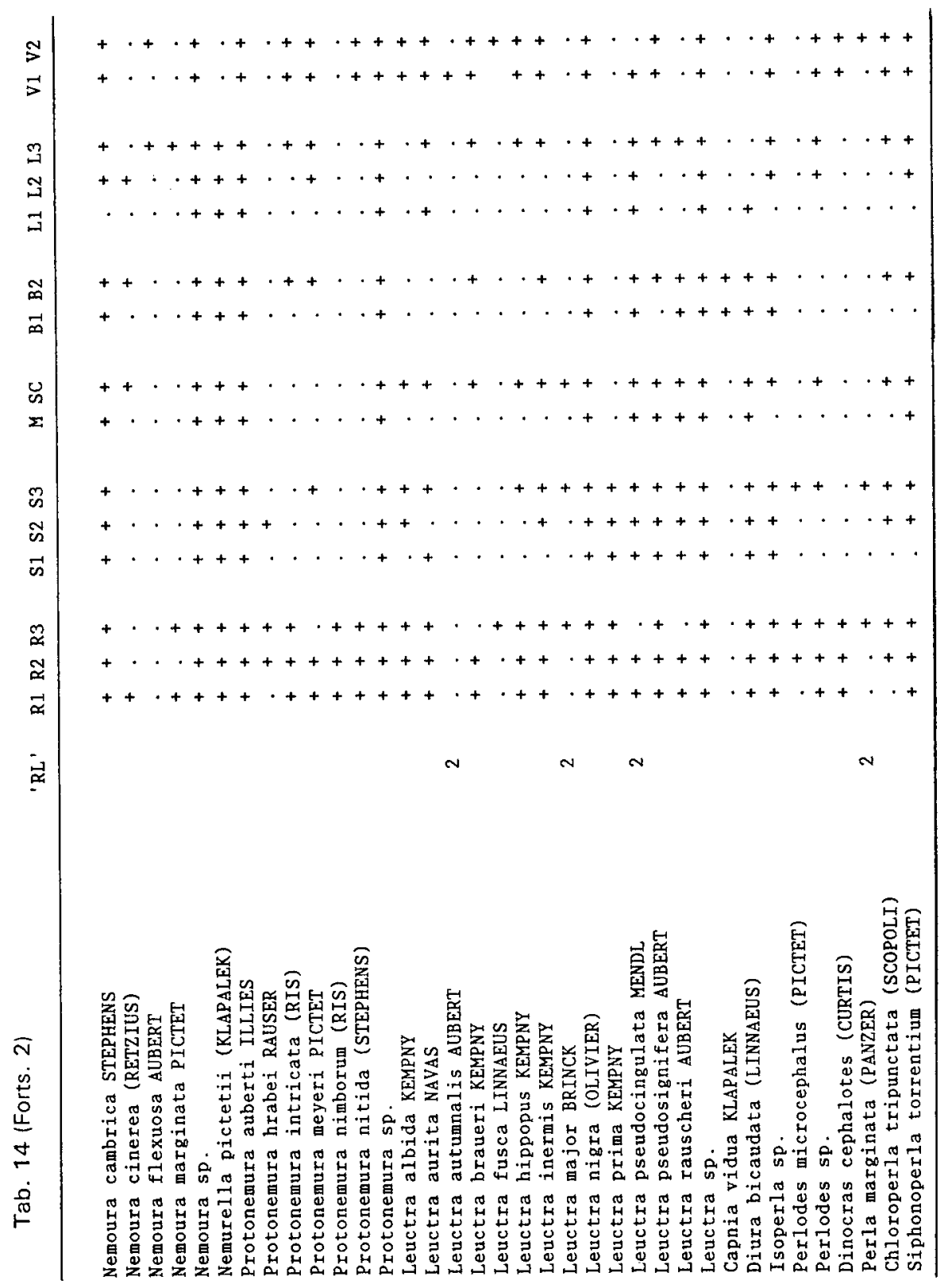




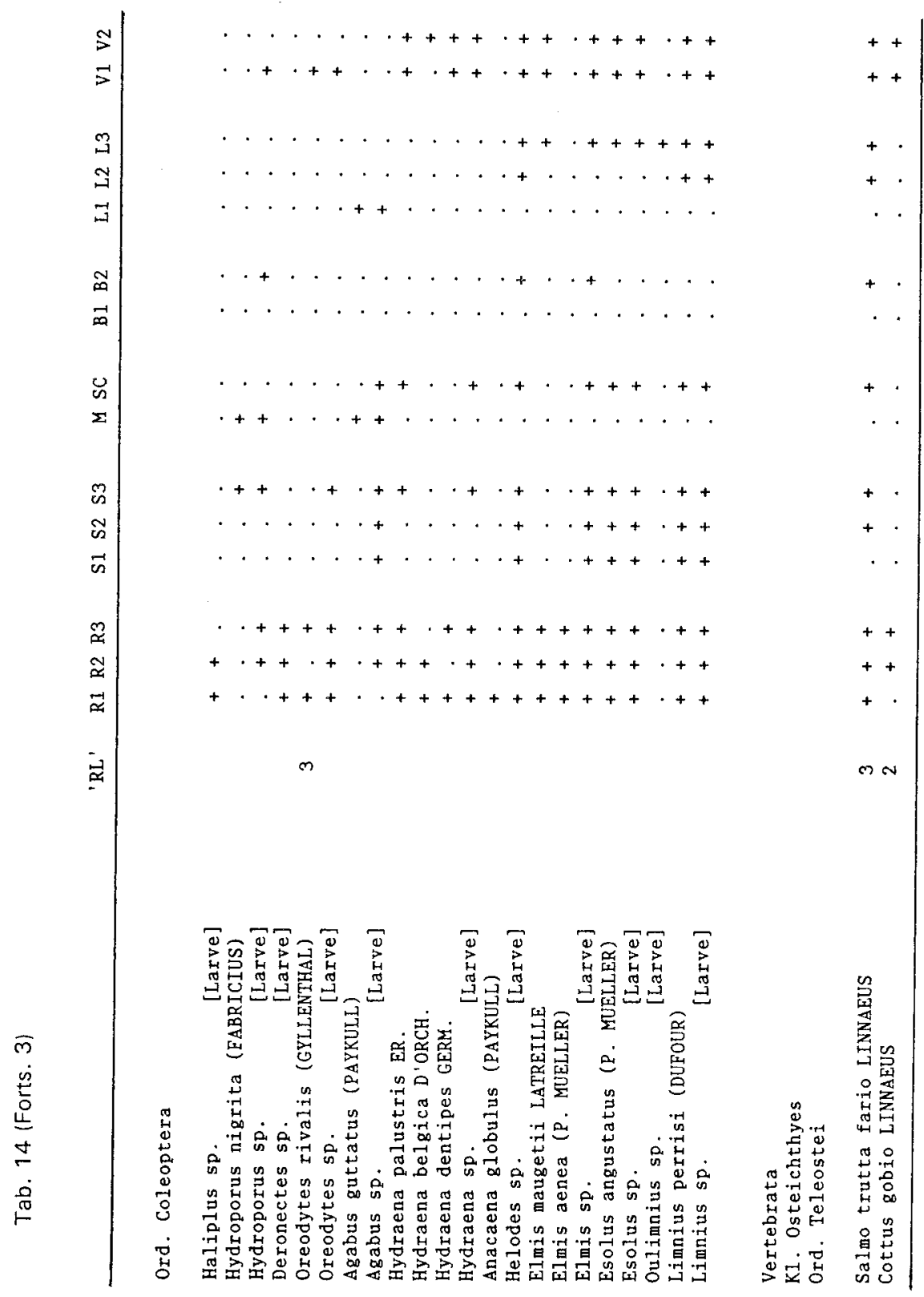


Tab. 15: Zaḥ der an den Probestellen nachgewiesenen Trichopteren-Arten (nach RÜDDENKLAU 1989)

\begin{tabular}{rrrrrrrrrrrrrrr}
$\mathrm{R} 1$ & $\mathrm{R} 2$ & $\mathrm{R} 3$ & $\mathrm{~S} 1$ & $\mathrm{~S} 2$ & $\mathrm{~S} 3$ & $\mathrm{M}$ & $\mathrm{SC}$ & $\mathrm{B} 1$ & $\mathrm{~B} 2$ & $\mathrm{~L} 1$ & $\mathrm{~L} 2$ & $\mathrm{~L} 3$ & $\mathrm{~V} 1$ & $\mathrm{~V} 2$ \\
\hline 30 & 35 & 27 & 8 & 18 & 6 & 6 & 14 & 9 & 13 & 8 & 13 & 21 & 22 & 24 \\
\hline
\end{tabular}

Zwischen der Gesamtartenzahl der bei dieser Untersuchung bearbeiteten Tiergruppen an den einzelnen Probestellen und deren mittlerem $\mathrm{pH}$-Wert besteht eine sehr hohe positive Korrelation ( $r=0,91, p<0,001$ ) (Abb. 54). Diese hohe Korrelation von Artenzahl und $\mathrm{pH}$-Wert ist bei allen Gruppen festzustellen (p stets $<0,001$ ):

$\begin{array}{lll}\text { Mollusca } & r=0,83 & \text { (Abb. 55) } \\ \text { Amphipoda } & r=0,82 & \text { (Abb. 56) } \\ \text { Ephemeroptera } & r=0,89 & \text { (Abb. 57) } \\ \text { Plecoptera } & r=0,86 & \text { (Abb. 58) } \\ \text { Coleoptera } & r=0,83 & \text { (Abb. 59) } \\ \text { Pisces } & r=0,86 & \text { (Abb. 60) }\end{array}$

Dabei errechnet sich nur bei den Molluscen $(+0,07)$, Coleopteren $(+0,02)$ und Amphipoden $(+0,02)$ ein geringfügig höherer Korrelationskoeffizient zu den minimal gemessenen $\mathrm{pH}$-Werten als zum mittleren $\mathrm{pH}$-Wert.

Sehr konstant ist der Anteil der drei artenreichsten Gruppen an der Gesamtartenzahl in den nicht versauerten Bächen (Abb. 61). In der Alten Riefensbeek und im Varleybach stellen die Plecopteren im Vergleich der fünf Probestellen 47 - 52 $\%$, die Ephemeropteren 21 - $25 \%$ und die Coleopteren $17-21 \%$ der Gesamtartenzahl. An allen drei Probestellen der Alten Riefensbeek haben die Plecopteren einen Anteil von $47 \%$. Mit abnehmenden mittleren $\mathrm{pH}$-Werten nimmt der Anteil der Plecopteren an der Gesamtartenzahl bis auf über $70 \%$ vor allem auf Kosten der Ephemeropteren an den am stärksten versauerten Probestellen zu. Auch bei den Coleopteren ist der Trend einer Abnahme zu beobachten. Insgesamt ist die Entwicklung aber uneinheitlicher als bei den Ephemeropteren, bei denen es mit zunehmender Wasserstoffionenkonzentration schließlich zum völligen Verschwinden der Gruppe kommt, wohingegen Coleopteren an einzelnen 


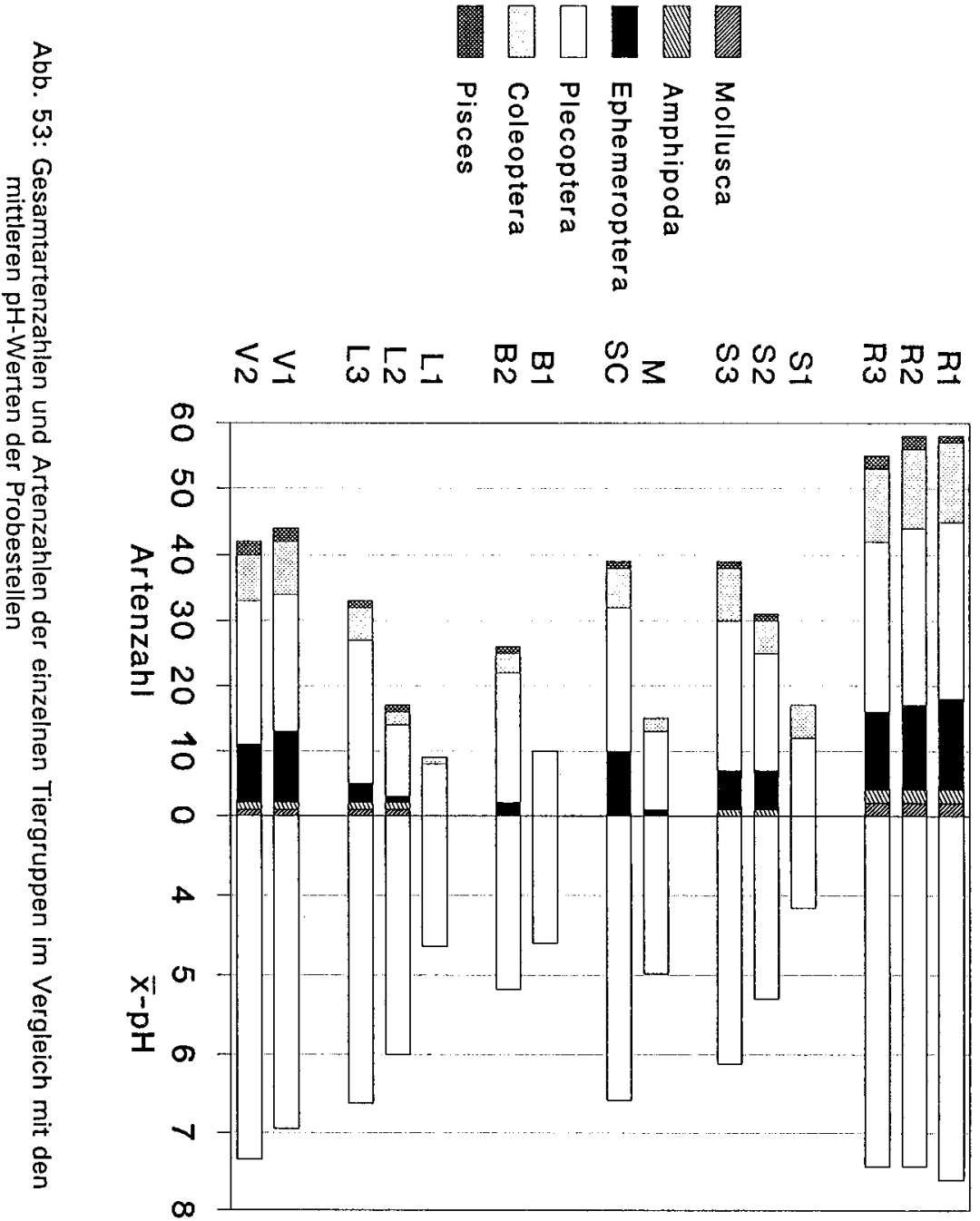


sehr sauren Stellen auch noch relativ hohe Anteile an der Gesamtartenzahl erreichen können.

Im Hinblick auf die Bedeutung der Probestellen für den Artenschutz zeigt sich bei der Analyse der in der Roten Liste für die Bundesrepublik Deutschland aufgeführten Arten und deren Gefährdungskategorie (BLAB et al. 1984), daß an den nicht versauerten Stellen von 12 insgesamt nachgewiesenen Rote Liste-Arten jeweils zwischen 5 und 7 gefährdete Arten vorkommen. An R3, V1 und $V_{2}$ sind darunter je 3 Arten der Gefährdungskategorie 2 "stark gefährdet". Von den versauerten Probestellen hat nur S3 eine vergleichbar hohe Zahl gefährdeter Arten. Mit zunehmender Versauerung nimmt deren Zahl parallel zur Gesamtartenzahl stark $a b$, so daß an den stark versauerten Stellen mit Leuctra pseudocingulata nur eine Rote Liste-Art zu finden ist.

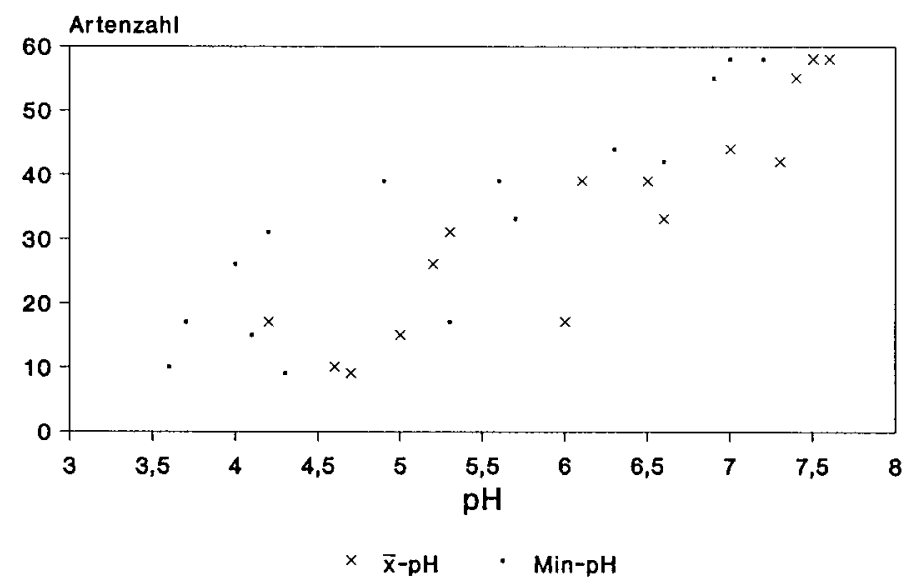

Abb. 54: Abhängigkeit der Gesamtartenzahl vom pH-Wert 


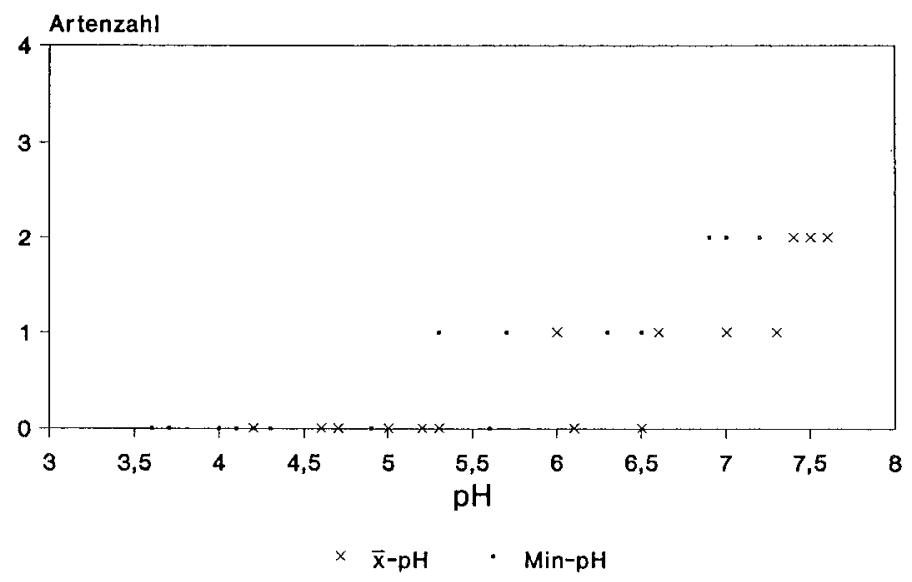

Abb. 55: Abhängigkeit der Anzahl der vorkommenden Molluscen-Arten vom $\mathrm{pH}$-Wert

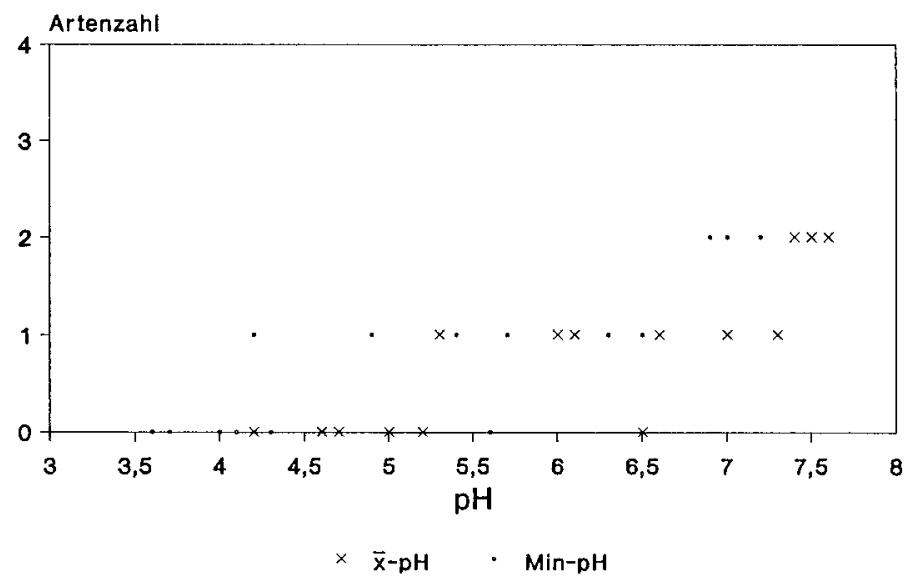

Abb. 56: Abhängigkeit der Anzahl der vorkommenden Amphipoden-Arten vom $\mathrm{pH}$-Wert 


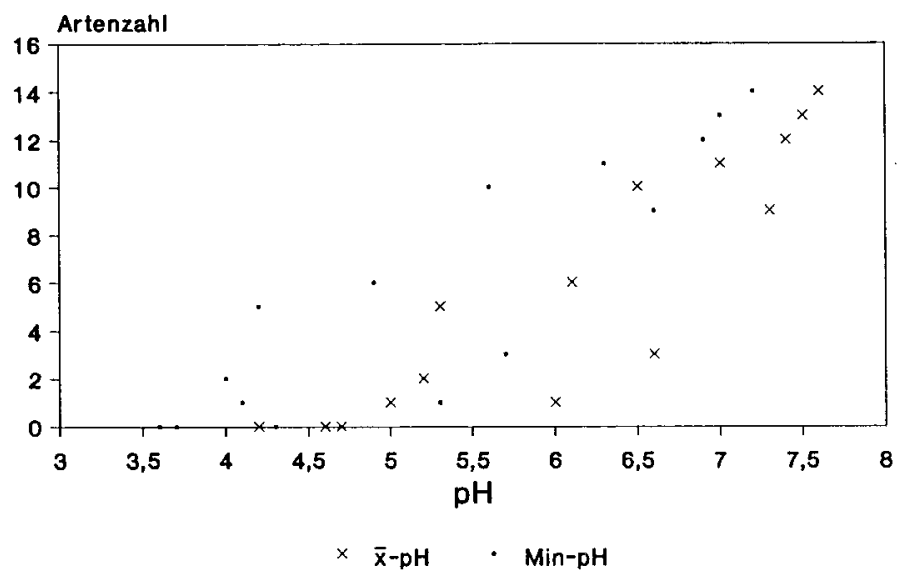

Abb. 57: Abhängigkeit der Anzahl der vorkommenden Ephemeropteren-Arten vom pH-Wert

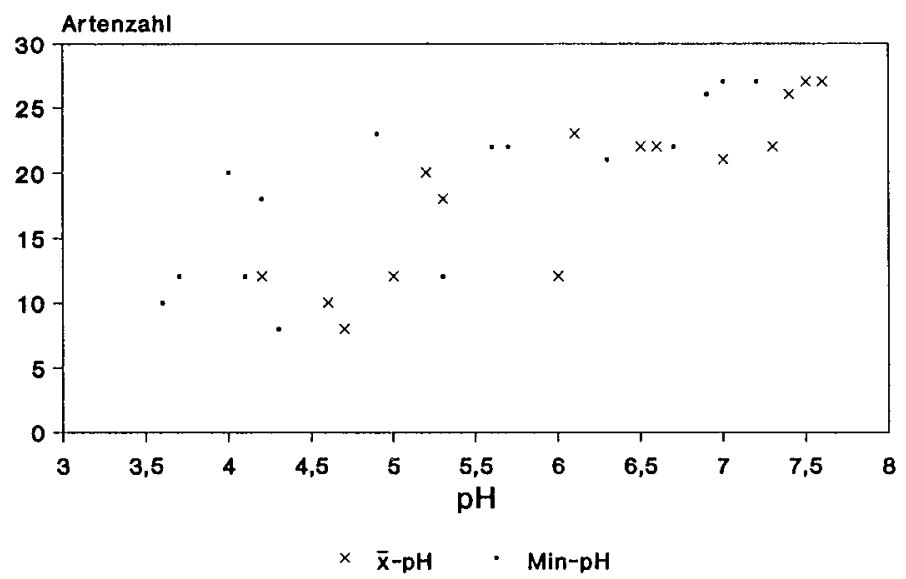

Abb. 58: Abhängigkeit der Anzahl der vorkommenden Plecopteren-Arten vom $\mathrm{pH}$-Wert 


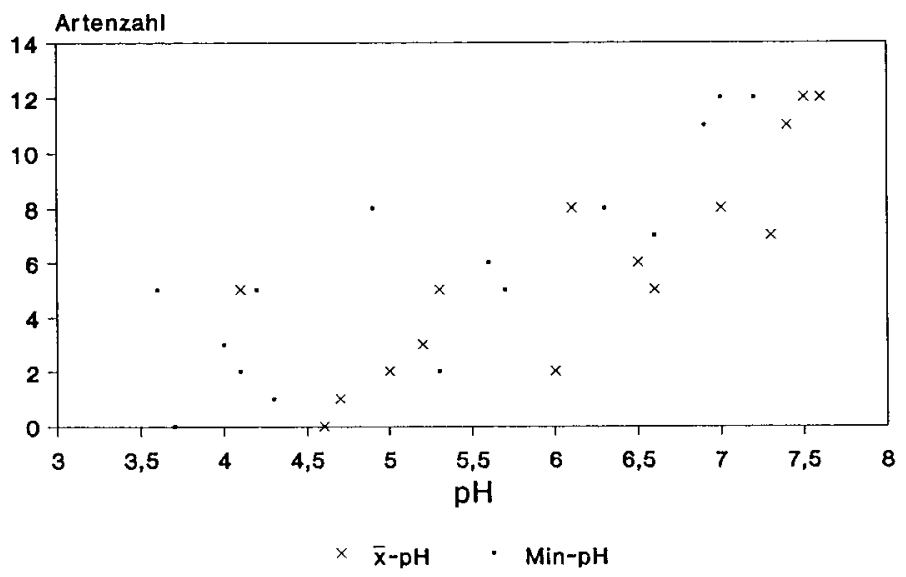

Abb. 59: Abhängigkeit der Anzahl der vorkommenden Coleopteren-Arten vom $\mathrm{pH}$-Wert

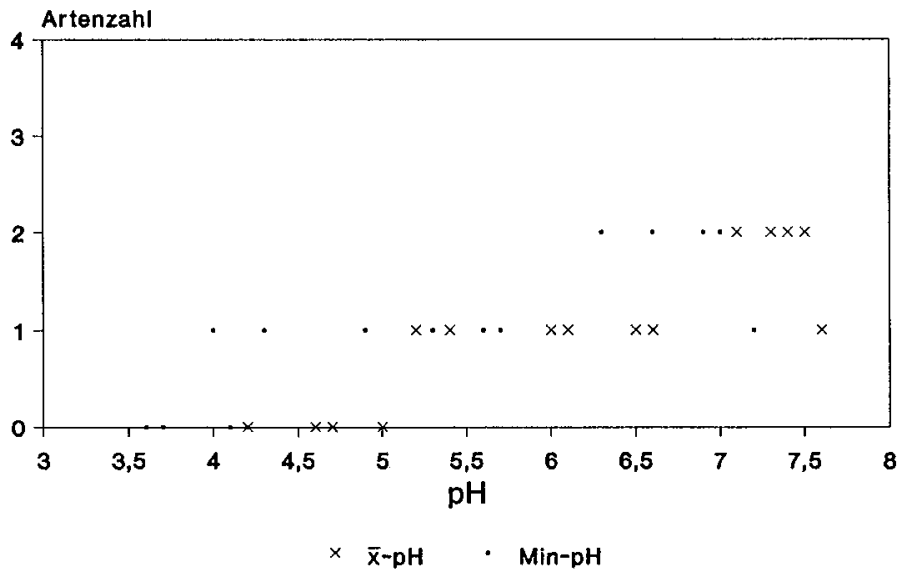

Abb. 60: Abhängigkeit der Anzahl der vorkommenden Fisch-Arten vom pH-Wert 


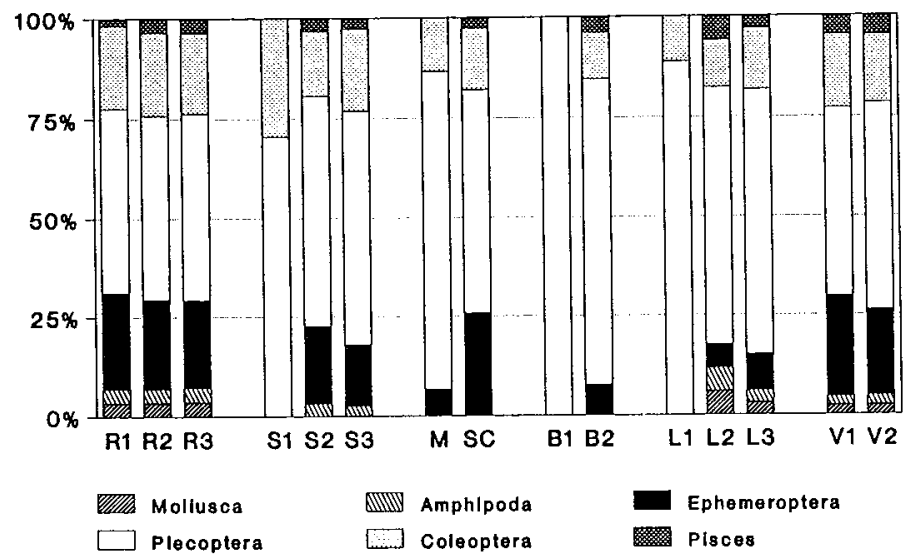

Abb. 61: Anteile der einzelnen Tiergruppen an den Gesamtartenzahlen der Probestellen 


\subsection{Autökologische Ergebnisse zur Versauerungstoleranz}

Durch die Verknüpfung der Verbreitung der einzelnen Arten an den Probestellen des Untersuchungsgebietes mit den Ergebnissen der abiotischen Untersuchungen lassen sich eine Reihe von autökologischen Daten gewinnen. Im Rahmen der vorliegenden Arbeit soll in erster Linie auf die Ergebnisse eingegangen werden, die im Zusammenhang mit der Betrachtung der Auswirkungen der Gewässerversauerung von Interesse sind. Fundierte Aussagen, die auf einer großen Zahl von Messungen beruhen, können dabei insbesondere zur Toleranz einzelner Arten gegenüber erhöhten Wasserstoffionenkonzentrationen gemacht werden (Tab. 16). Bei der Auflistung der mittleren tolerierten pH-Werte wurden nur die Probestellen berücksichtigt, an denen auch eine Reproduktion festgestellt werden konnte. Als minimal tolerierte $\mathrm{pH}$-Werte werden dagegen diejenigen Werte angeführt, bei denen Individuen der jeweiligen Art lebend im Gewässer angetroffen wurden bzw. zum Zeitpunkt der pH-Messung aufgrund des Abundanzganges dort sein mußten.

Da mit dem pH-Wert eine Reihe weiterer Parameter korreliert sind, können auch auf diese Rückschlüsse gezogen werden (s. Kap. 5.1.6.1). Ein besonderes Interesse kommt den Aluminium-Konzentrationen zu, von denen bekannt ist, daß sie zumindest für einige Arten ein zusätzlicher und teilweise auch entscheidender Belastungsfaktor sein können. Mit Hilfe der Ergebnisse der relativ wenigen Speziierungsanalysen läßt sich jedoch nur ein sehr grobes Bild der tatsächlich tolerierten Konzentrationen zeichnen, so daß die angegebenen Werte nur der Orientierung dienen können.

Die in Ergänzung der Freilandbeobachtungen von AVERMANN (1990a) durchgeführten laborexperimentellen Untersuchungen zur Säure- und Aluminiumtoleranz, auf die bei den untersuchten Arten näher eingegangen wird, sind ebenfalls nur als Einstieg in die Problematik der Reaktion ausgewählter Arten auf die Mehrfachbelastung durch die Gewässerversauerung zu sehen. 
Tab. 16: PH-Toleranzen häufiger Arten des Untersuchungsgebietes in Verbindung mit den mittleren Calcium-Konzentrationen und den gemessenen Maximum-Konzentrationen anorganischer Aluminium-Spezies (weitere Einzelheiten s. Text)

\begin{tabular}{|c|c|c|c|c|}
\hline Art & $\overline{\mathrm{x}}-\mathrm{pH}$ & $\mathrm{Min}-\mathrm{pH}$ & $\begin{array}{r}\bar{x}-\mathrm{Ca} \\
{[\mathrm{mg} / 1]}\end{array}$ & $\begin{array}{r}\text { Max- } \\
\text { A1 anorg } \\
{[\text { mg } / 1]}\end{array}$ \\
\hline Ancylus fluviatilis & 6,9 & 6,2 & 11,2 & $<0,01$ \\
\hline Gammarus fossarum & 6,9 & 6,2 & 11,2 & $<0,01$ \\
\hline G. pulex & 7,4 & 6,9 & 12,3 & $<0,01$ \\
\hline Baetis alpinus & 6,6 & 5,6 & 6,1 & 0,28 \\
\hline B. muticus & 6,6 & 5,6 & 6,1 & 0,28 \\
\hline B. rhodani & 6,1 & 5,2 & 4,1 & 0,21 \\
\hline B. vernus & 5,3 & 4,3 & 4,1 & 0,36 \\
\hline Epeorus sylvicola & 6,6 & 5,0 & 5,3 & 0,39 \\
\hline Rhithrogena semicolorata & 6,6 & 5,3 & 5,3 & 0,39 \\
\hline Ecdyonurus venosus & 6,6 & 5,6 & 6,1 & 0,28 \\
\hline Ephemerella ignita & 6,9 & 6,2 & 11,2 & $<0,01$ \\
\hline E. mucronata & 6,9 & 6,2 & 11,2 & $<0,01$ \\
\hline Habroleptoides modesta & 6,9 & 6,2 & 11,2 & $<0,01$ \\
\hline Habrophlebia lauta & 6,9 & 5,8 & 8,4 & 0,12 \\
\hline Brachyptera seticornis & 5,3 & 4,2 & 4,1 & 0,73 \\
\hline Amphinemura standfussi & 4,2 & 3,7 & 2,0 & 1,05 \\
\hline A. sulcicollis & 5,2 & 4,0 & 3,5 & 0,58 \\
\hline Nemoura cambrica & 4,2 & 3,7 & 2,0 & 1,05 \\
\hline Nemure1la pictetii & 4,2 & 3,7 & 2,0 & 1,05 \\
\hline Protonemura auberti & 4,2 & 3,7 & 2,0 & 1,05 \\
\hline P. inticata & 5,2 & 4,0 & 3,5 & 0,53 \\
\hline P. meyeri & 5,2 & 4,3 & 3,5 & 0,53 \\
\hline P. nimborum & 7,4 & 6,9 & 12,3 & $<0,01$ \\
\hline P. nitida & 6,9 & 6,2 & 11,2 & $<0,01$ \\
\hline Leuctra albida & 5,3 & 4,2 & 4,1 & 0,58 \\
\hline L. aurita & 4,2 & 3,7 & 2,0 & 1,05 \\
\hline L. braueri & 5,2 & 4,0 & 3,5 & 0,53 \\
\hline L. fusca & 7,3 & 6,6 & 12,3 & $<0,01$ \\
\hline L. hippopus & 6,1 & 4,9 & 5,3 & 0,39 \\
\hline L. inerris & 5,2 & 4,0 & 3,5 & 0,58 \\
\hline L. major & 6,1 & 4,9 & 5,3 & 0,39 \\
\hline L. nigra & 4,2 & 3,7 & 2,0 & 1,05 \\
\hline L. prima & 4,2 & 3,7 & 2,0 & 1,05 \\
\hline L. pseudocingulata & 4,2 & 3,7 & 2,0 & 1,05 \\
\hline L. pseudosignifera & 4,2 & 3,7 & 2,0 & 1,05 \\
\hline L. rauscher $i$ & 4,2 & 3,7 & 2,0 & 1,05 \\
\hline Capnia vidua & 4,6 & 3,7 & 2,2 & 0,53 \\
\hline Diura bicaudata & 4,2 & 3,7 & 2,0 & 1.05 \\
\hline Dinocras cephalotes & 6,9 & 6,2 & 11,2 & $<0,01$ \\
\hline Ch1oroperla tripunctata & 6,1 & 4,9 & 3,5 & 0,39 \\
\hline Siphonoperla torrentium & 5,0 & 4,2 & 3,7 & 1,05 \\
\hline Agabus guttatus & 4,2 & 3,7 & 2,0 & 1,05 \\
\hline Hydraena palustris & 6,1 & 4,9 & 5,3 & 0,39 \\
\hline Elmis maugetii & 6,6 & 4,2 & 4,1 & 0.58 \\
\hline Esolus angustatus & 6,1 & 4,2 & 2,0 & 0,45 \\
\hline Limnius perrisi & 5,8 & 4,2 & 2,0 & 0,70 \\
\hline Salmo trutta fario & 6,1 & 4,9 & 5,3 & 0,39 \\
\hline Cottus gobio & 6,9 & 6,2 & 11,2 & $<0,01$ \\
\hline
\end{tabular}




\subsubsection{Mollusca}

Silikat-Bergbäche werden allgemein von nur sehr wenigen Mollusken-Arten besiedelt, was in ihrer für die Tiergruppe ungünstigen morphologisch-ökologischen Struktur mit hohen Fließgeschwindigkeiten, grobem Substrat und fehlenden Makrophyten begründet liegt (BRAUKMANN 1987). Mit den beiden nachgewiesenen Arten Ancylus fluviatilis und Pisidium $\mathrm{sp}$. ist daher das Spektrum der in Harzer Bergbächen vorkommenden Arten, wie der Vergleich mit anderen Untersuchungen zeigt, bereits weitgehend ausgeschöpft (HEITKAMP et al. 1985).

Ancylus fluviatilis: Als einzige Mollusken-Charakterart des Epirhithrals ist Ancylus fluviatilis morphologisch und physiologisch an turbulente Strömung, grobes Substrat und niedrige Wassertemperaturen angepaßt (ILLIES 1952). Die Art konnte in niedrigen Abundanzen an allen nicht versauerten Probestellen nachgewiesen werden. Nach SUTCLIFFE \& CARRICK (1973) kommt Ancylus bis pH 5,5 vor. In Mitteleuropa wird sie auch in anderen Gebieten bei niedrigen Calcium-Konzentrationen erst bei $\mathrm{pH}-$ Werten, die deutlich über 6,0 liegen, gefunden: > 6,3 (LESSMANN 1983), > 6,4 (MATTHIAS 1983), > 6,8 (SCHÖLL 1987), > 6,5 (WESTERMANN 1990). Im Sauerland ist die Art in den letzten Jahrzehnten aus vielen versauerungssensitiven Gewässern verschwunden (SCHIMMER 1987). Bei $\mathrm{pH}$-Werten < 7,0 beginnt die kalkhaltige Schale sich aufzulösen (SINGER 1982).

Pisidium sp: Nachweise von Pisidien mit sehr niedrigen Abundanzen von nur einzelnen Individuen pro $\mathrm{m}^{2}$ stammen aus der nicht versauerten Alten Riefensbeek und der Langen Bramke. Das Vorkommen der Gattung bis zu pHWerten von 4,2 in anderen Gewässern (NILSSEN 1980, ÖKLAND \& ÖKLAND 1986. WESTERMANN 1990) und das Fehlen im Varleybach weisen darauf hin, daß die Verbreitung im Harz nicht in erster Linie durch die Wasserstoffionenkonzentration begrenzt sein muß.

\subsubsection{Amphipoda}

Als omnivore Zerkleinerer groben organischen Materials, insbesondere von Fallaub, sind die Gammariden ein wichtiges Nahrungskettenglied der Fließgewässer (BREHM \& MEIJERING 1982). 
Gammarus fossarum / Gammarus pulex: Gammariden können als gute Bioindikatoren der Gewässerversauerung angesehen werden, da sie ausschließlich in den beiden nicht versauerten Bächen gefunden werden können. Gammarus fossarum kommt im Varleybach bis zu einem minimalen $\mathrm{pH}$ von 6,2 und Gammarus pulex in der Alten Riefensbeek bis zu einem minimalen $\mathrm{pH}$ von 6,9 vor. Die mittleren Calcium-Konzentrationen liegen an allen Stellen über $11 \mathrm{mg} / \mathrm{l}$ und die mittleren Magnesium-Gehalte über $2,8 \mathrm{mg} / \mathrm{l}$. Die Konzentrationen anorganischen monomeren Aluminiums bleiben stets unter $0,01 \mathrm{mg} / \mathrm{l}$.

Bei ergänzenden laborexperimentellen Untersuchungen (AVERMANN 1990a) mit beiden Arten kam es bei pH 5,0 binnen 48 Stunden zu keiner Erhöhung der Mortalitätsraten. Bei pH 4,5 wurde nach 24 - 36 Stunden für beide Arten eine Mortalitätsrate von $50 \%$ festgestellt, die sich bei pH 4,0 nach $36-48$ Stunden auf $100 \%$ erhöhte. Versuche unter Zugabe von 1 und $2 \mathrm{mg} / / \mathrm{Al}^{3+}$ erbrachten keine zusätzliche Erhöhung der Mortalitätsrate, was die Ergebnisse anderer Untersuchungen weitestgehend bestätigt (MEINEL et al. 1985, ORMEROD et al. 1987b, BAUER et al. 1988). Eine Mortalitätserhöhung war erst unter Zusatz von $3 \mathrm{mg} / \mathrm{Al}{ }^{3+}$ nach mehr als vier Tagen bei pH 5,5 zu verzeichnen (BAUER et al. 1988).

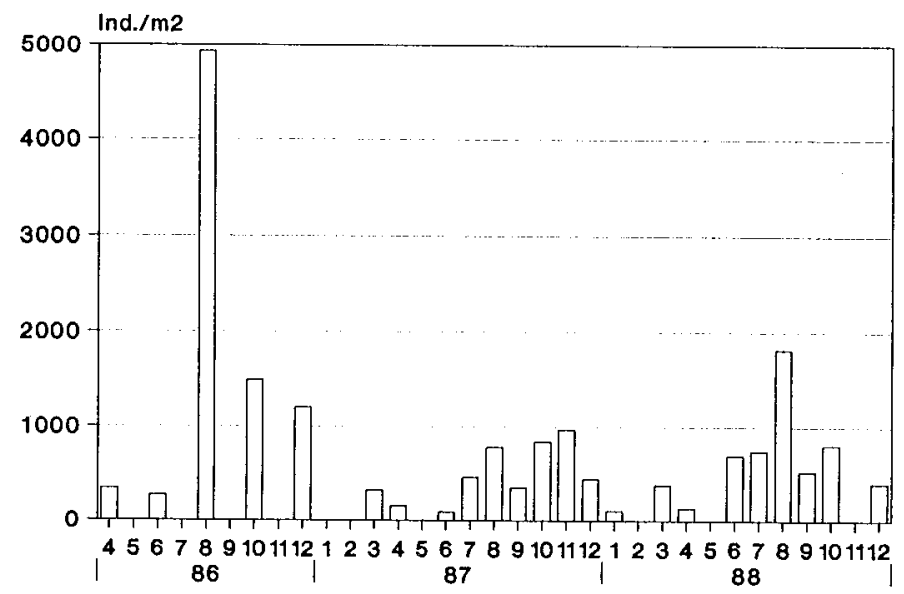

Abb. 62: Abundanzen von Gammarus pulex an R1 
Aus Freilandbeobachtungen wird dagegen auf unterschiedliche Säuretoleranzen geschlossen. So wird Gammarus fossarum unter $\mathrm{pH} 6$ nur sehr selten gefunden (MEINEL et al. 1985, ÖKLAND \& ÖKLAND 1986). Die als konkurrenzstärker geltende Art Gammarus pulex kann auch an schwach sauren Stellen vereinzelt bis pH 5 auftreten (BREHM \& MEIJERING 1982, WESTERMANN 1990).

Abweichend von der Beobachtung einer typischen höhenzonalen Verbreitung der Gammariden mit dem alleinigen Vorkommen von Gammarus fossarum oberhalb von $450 \mathrm{~m}$ und einem Auftreten von Mischpopulationen mit Gammarus pulex in darunter gelegenen Höhen (MEIJERING 1971, MEIJERING \& PIEPER 1982), konnte im Harz bisher stets Gammarus pulex an den am höchsten gelegenen Stellen beobachtet werden. So kommt ausschließlich diese Art in über $600 \mathrm{~m}$ Höhe in dem westlich von Braunlage auf einer Hochfläche fließenden Brunnenbach vor (LESSMANN unveröff.). In der Sieber wurde Gammarus pulex als einzige Gammariden-Art oberhalb einer Mischpopulation aus beiden Arten gefunden, was hier auch für eine größere Säuretoleranz der Art unter Freilandbedingungen sprechen kann (LESSMANN 1983, HEITKAMP et al. 1985).

In der Alten Riefensbeek erreicht Gammarus pulex an R1 in einer Höhe von $\mathbf{5 4 0}$ $\mathrm{m}$ im Mittel Dichten von $790 \mathrm{Ind} . / \mathrm{m}^{2}$, an R2 tritt er mit $160 \mathrm{Ind} . / \mathrm{m}^{2}$ auf und hat an R3 nur noch 13 Ind. $/ \mathrm{m}^{2}$. Die Besiedlungsdichte weist im Jahresverlauf regelmäßig erhebliche Schwankungen auf (Abb. 62). An R1 war in jedem der Untersuchungsjahre nach einem Minimum im Winter und Frühjahr ein steiler Anstieg der Dichten im Sommer zu beobachten, wobei 1986 und 1988 bereits im August das Maximum erreicht wurde. 1987 und 1988 zeigte sich nach einem ersten stärkeren Rückgang im September ein nochmaliger Anstieg im Herbst, dem das starke Absinken der Besiedlungsdichten im Winter folgte. Im Varleybach fehlt Gammarus pulex vollständig. Gammarus fossarum kommt hier mit im Mittel $36 \mathrm{Ind} . / \mathrm{m}^{2}$ an $\mathrm{V} 1$ und mit nur einzelnen Tieren an $\mathrm{V} 2$ vor.

Abgesehen von der Wasserstoffionenkonzentration werden für die Verbreitung der Gammariden-Arten vor allem unterschiedliche Strömungsresistenzen bei gleichgerichteter Abnahme der Empfindlichkeit gegen Sauerstoffmangel sowie zunehmender Resistenz gegenüber höheren Wassertemperaturen von Gammarus fossarum zu Gammarus pulex verantwortlich gemacht (MEIJERING 1971, PINKSTER 1972). Da Sauerstoffgehalt und Wassertemperatur in den untersuchten Harzbächen kaum Unterschiede aufweisen, ist insbesondere die Fließgeschwindigkeit als ein wichtiger verbreitungsregulierender Faktor anzusehen, der 
Gammarus pulex neben der geringen Beschattung auch das Vorkommen mit hohen Abundanzen an der von der Choriotop-Struktur her von den anderen Probestellen mit Gammariden-Vorkommen abweichenden Probestelle R1 ermöglicht. Die Abundanz liegt an dieser Stelle weit über sonst angetroffenen Dichten in Silikat-Bergbächen (BRAUKMANN 1987).

Niphargus sp.: Niphargus cf. aquilex ist eine typische Art grundwasserbeeinflußter Gewässerbereiche (SCHELLENBERG 1942), die an allen Probestellen der Alten Riefensbeek und an den mittleren und unteren Stellen von Großer Söse und Langer Bramke gefunden wird. Abgesehen von L2 und S2 mit maximal rund 70 Ind./ $\mathrm{m}^{2}$ kommt Niphargus nur mit geringen Abundanzen vor. In der Alten Riefensbeek ist bachabwärts bei zurückgehender Gammariden-Besiedlung eine Zunahme der Abundanz zu verzeichnen. Die Tendenz eines gegenseitigen Ausschlusses von Niphargus und Gammarus ist seit längerem bekannt (DITTMAR 1955). In Bezug auf die Säuretoleranz ist festzustellen, daß die Art bis zu einem in der freien Welfe gemessenen Minimum-pH von 4,2 bei einem niedrigsten mittleren $\mathrm{pH}$ von 5,3 in den Untersuchungsgewässern vorkommt und an keiner der stark versauerten Probestellen gefunden werden konnte. Auf die Meidung sehr saurer Gewässer weist MEIJERING (mdl. Mitt.) hin. WESTERMANN (1990) fand Niphargus dagegen auch bei einem mittleren $\mathrm{pH}$ von 3,9.

\subsubsection{Ephemeroptera}

Ephemeropteren gehören mit den Plecopteren, Trichopteren und Dipteren zu den nach Artenzahl und Besiedlungsdichte wichtigsten Benthosorganismen in Bergbächen, die häufig aufgrund der großen Dominanz einzelner Arten zu bestimmten Zeiten des Jahres die quantitativ größte Tiergruppe stellen (BRAUKMANN 1987). Sie sind unter den Insektenordnungen diejenige, die am empfindlichsten auf die Gewässerversauerung reagiert und bei der es bereits in schwach sauren Gewässern zu einem Ausfall vieler Arten kommt (UBA 1987, MATTHIAS 1983, HEITKAMP et al. 1985, BAUER et al. 1987). Zu Schädigungen führen beispielsweise lonenverluste nach Häutungen und Aluminium-Ausfällungen auf den Respirationsorganen (McCAHON et al. 1987).

Siphlonurus aestivalis: Die Art gehört mit ihrem Vorkommen an den mäßig versauerten Probestellen S2 und B2 zu den wenigen relativ säuretoleranten Ephemeropteren, die an beiden Stellen jedoch mit im Mittel nur 1 Ind. $/ \mathrm{m}^{2}$ und 
ausschließlich in den Sommermonaten auftritt, nachdem die pH-Werte mehrere Monate über 5 gelegen haben. Bei Untersuchungen in Schweden hat die Art sich ebenfalls relativ säureunempfindlich gezeigt (HARMANEN 1980, OTTO \& SVENSSON 1983, ENGBLOM \& LINGDELL 1984).

Baetis: Mit sieben nachgewiesenen Arten stellt Baetis die individuenstärkste Ephemeropteren-Gattung der untersuchten Bäche. Sie ist an 12 der 15 Probestellen zu finden, wobei sie jedoch nur in den nicht versauerten Gewässern ein ganzjähriges, permanentes Auftreten zeigt (Abb. 63). Abgesehen von B2 kommt Baetis an den schwach und mäßig versauerten Stellen dagegen nur in den Sommer- und/oder Herbstmonaten vor (Abb. 64). Mit Baetis vernus und Baetis rhodani gelingt es nur zwei Arten, sich hier zu vermehren. Dementsprechend erreicht die Gattung in den neutralen Bächen mittlere Besiedlungśdichten zwischen 740 und $1100 \mathrm{Ind} . / \mathrm{m}^{2}$, wohingegen in den versauerten Gewässern maximal $540 \mathrm{Ind} . / \mathrm{m}^{2}$ gezählt werden konnten. Die Werte liegen für die meisten Stellen noch wesentlich niedriger. An B2, wo die DOC-Konzentrationen relativ hoch sind und ein größerer Teil des Aluminiums organisch gebunden ist, konnten auch im Frühjahr 1987 wenige Baetis-Larven gefunden werden.

Eine Art der versauerten Bereiche ist neben der allgemein verbreitetsten Art Baetis rhodani auch Baetis vernus, die hier die höchsten Besiedlungsdichten hat. Baetis scambus, Baetis alpinus und Baetis muticus treten neben den nicht versauerten Probestellen zusätzlich nur an den schwach versauerten Stellen auf. Baetis lutheri und Baetis melanonyx sind Arten, die ausschließlich in der Alten Riefensbeek vorkommen. Ergänzende laborexperimentelle Untersuchungen mit Baetis-Larven konnten wegen einer zu hohen Mortalität bereits bei der Hälterung nicht durchgeführt werden (AVERMANN 1990a).

Da Baetis-Weibchen ihre Eier nur in Gewässern ablegen, deren pH über 6,0 liegt, sind höhere $\mathrm{pH}$-Werte während der Emergenz eine wichtige Voraussetzung für einen Besiedlungsversuch versauerter Gewässern durch die Gattung (SUTCLIFFE \& CARRICK 1973). Die euryöke Baetis rhodani stellt relativ geringe Ansprüche an die Wasserqualität. Sie besiedelt Krenal bis Epipotamal und zeigt auch im $a$ mesosaproben Bereich noch Massenentwicklungen (BRAASCH \& JACOB 1976, MÜLLER-LIEBENAU 1969). In sehr elektrolytarmen Gewässern fehlt sie unter $\mathrm{pH}$ 5, kann sich bei höheren Wasserhärten aber noch bei $\mathrm{pH} \mathrm{4,5} \mathrm{bis} \mathrm{4,7} \mathrm{halten}$ (WILLOUGHBY 1988). Das Auftreten von Baetis vernus in großer Zahl in den Sommermonaten in nur periodisch stärker sauren Gewässern wurde schon 


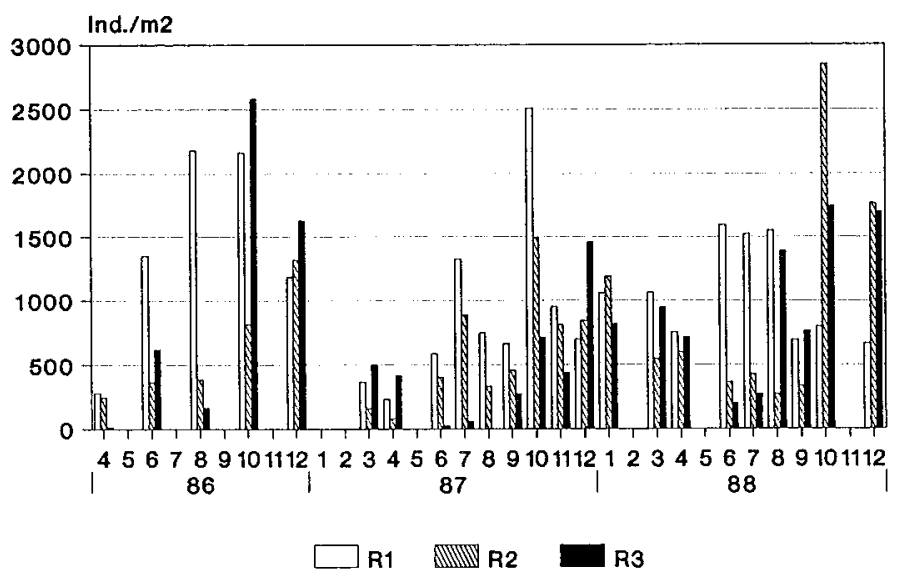

Abb. 63: Benthos-Abundanzen von Baetis spp. in der Alten Riefensbeek

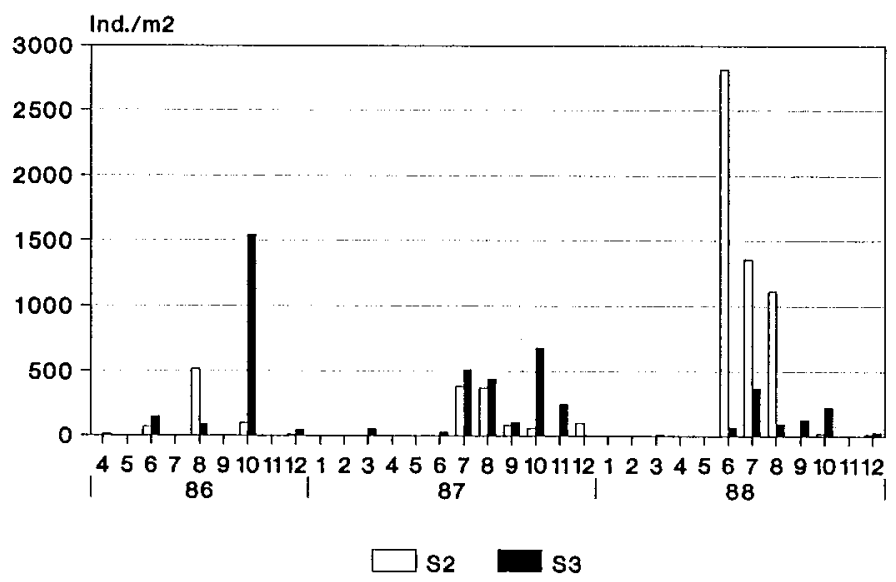

Abb. 64: Benthos-Abundanzen von Baetis spp. in der Großen Söse 
mehrfach beobachtet (LESSMANN 1983, HEITKAMP et al. 1985, STONER et al. 1984, WESTERMANN 1990). Baetis vernus ist die Besiedlung dieser vor allem im Winterhalbjahr längere Zeit niedrige $\mathrm{pH}$-Werte aufweisenden Bereiche möglich, da ihre Eier im Winter eine Diapause einlegen (THORUP et al. 1987, BOHLE 1969). Aufgrund des verminderten Konkurrenzdrucks kann es deshalb im Sommerhalbjahr leicht zu Massenentwicklungen kommen. Baetis rhodani kann in diesen Gewässerbereiche dagegen keine beständige Besiedlung erreichen. Die Art hat eine langsam wachsende Wintergeneration und eine schnell wachsende Sommergeneration (BENGTSSON 1988), von denen sich nur die Sommergeneration in versauerten Gewässern, die im Sommerhalbjahr höhere $\mathrm{pH}-$ Werte aufweisen, entwickeln kann.

Für Baetis scambus ist eine Säuretoleranz bis $\mathrm{pH} \mathrm{5,2} \mathrm{bekannt} \mathrm{(WILLOUGHBY}$ 1988). $\mathrm{Ab} \mathrm{pH}<5,5 \mathrm{kommt}$ es zu einer starken Erhöhung der Mortalität nach Häutungen (HAVAS \& HUTCHINSON 1983). Der Art wird deshalb meistens nur in circumneutralen Gewässern gefunden (HARMANEN 1980, WESTERMANN 1990). Eine hohe Säureempfindlichkeit ist ebenfalls für Baetis alpinus, Baetis lutheri und Baetis melanonyx beschrieben worden (WESTERMANN 1990).

Heptageniidae: Die Larven von Epeorus sylvicola, Rhithrogena semicolorata und Ecdyonurus venosus gehören zu den typischen Besiedlern aller nicht versauerten Probestellen. Epeorus sylvicola zeigt mit im Mittel 4 bis 48 Ind. $/ \mathrm{m}^{2}$ die niedrigsten Besiedlungsdichten. Die Ecdyonurus-Arten kommen in der Alten Riefensbeek im Mittel aller Probenahmen auf 40 bis $66 \mathrm{Ind} . / \mathrm{m}^{2}$, wohingegen im Varleybach 139 bzw. 72 Ind. $/ \mathrm{m}^{2}$ erreicht werden. Rhithrogena semicolorata hat in beiden Bächen mit 60 bis 160 Ind./ $\mathrm{m}^{2}$ eine etwa gleich starke Besiedlung. An den versauerten Stellen liegt die mittlere Dichte bei einem kurzzeitigen Vorkommen ausschließlich bei neutralen bis schwach sauren $\mathrm{pH}$-Werten in den Herbstmonaten und gelegentlich im Sommer stets unter $20 \mathrm{lnd} . / \mathrm{m}^{2}$, meist sogar deutlich unter $10 \mathrm{Ind} . / \mathrm{m}^{2}$.

Die als typische Bergbachbewohner geltenden rheophilen und kaltstenothermen im Harz vorkommenden Arten der Heptageniidae sind alle sehr säureempfindlich und wurden auch bei anderen Untersuchungen bisher nur bei $\mathrm{pH}$-Werten über 6,0 gefunden (ZIEMANN 1975, BÜRK 1979, MATTHIAS 1983, HEITKAMP et al. 1985, ORMEROD et al. 1987b, MCCAHON 1987). 
Ergänzende laborexperimentelle Untersuchungen erbrachten für Epeorus sylvicola eine starke Erhöhung der Mortalitätsrate unter zusätzlicher Aluminiumbelastung bei niedrigen pH-Werten (AVERMANN 1990a). Wurde bei $\mathrm{pH} 3,5$ nach 48 Stunden nur eine Gesamtmortalität von $76 \%$ registriert, kam es bei Zugabe von $1 \mathrm{mg} / / \mathrm{Al}^{3+}$ bereits bei $\mathrm{pH} 4,0 \mathrm{zu}$ einer Mortalitätsrate von $50 \%$ und mit $2 \mathrm{mg} / \mathrm{Al}{ }^{3}+$ wurde schon bei $\mathrm{pH} \mathrm{5,0}$ nach 12 Stunden eine Mortalität von $50 \%$ registriert, die sich bei pH 3,5 nach 24 Stunden auf $100 \%$ erhöhte. Für Ecdyonurus venosus ergab sich dagegen nur eine relativ geringe Steigerung der Mortalitätsrate unter Al-Zusatz von $55 \%$ ohne Al-Zusatz bei $\mathrm{pH}$ 3,5 auf 69 $\%$ und $84 \%$ bei 1,36 bzw. $2,67 \mathrm{mg} / / \mathrm{Al}^{3+}$.

Ephemerellidae: Ephemerella ignita und Ephemerella mucronata kommen in den nicht versauerten Bächen mit mittleren Abundanzen bis etwa 100 Ind. $/ \mathrm{m}^{2}$ vor. Beide Arten fehlten ebenfalls bei zahlreichen anderen Untersuchungen in Gewässern, die einen pH-Wert von 6,0 unterschreiten (ZIEMANN 1975, MATTHIAS 1983, ENGBLOM \& LINGDELL 1984, STONER et al. 1984, WESTERMANN 1990).

Leptophlebiidae: Paraleptophlebia submarginata wurde nur an S2 in der Emergenz nachgewiesen. Die Art kommt meist nicht in versauerten Bereichen vor (SANDROCK 1978, HARMANEN 1980, MATTHIAS 1983). Sie ist eine häufige Art des niedersächsischen Tieflands (LEHRKE \& REUSCH 1987).

Habroleptoides modesta und Habrophlebia lauta sind außerhalb der neutralen Gewässer nur zeitweilig an SC in sehr geringer Dichte zu finden. Der Besiedlungsschwerpunkt von Habrophlebia lauta liegt an den beiden quellfernsten Probestellen. Im Laborexperiment konnte gezeigt werden, daß Habroleptoides modesta zu den Arten gehört, die bei einer Versauerung auf zusätzliche erhöhte Aluminium-Konzentrationen mit einer ansteigenden Mortalitätsrate reagieren (AVERMANN 1990a). Unter alleiniger Protonenbelastung wurde eine Mortalitätsrate von $25 \%$ bei $\mathrm{pH} 3,5$ nach 48 Stunden registriert. Bei $1,36 \mathrm{mg} / / \mathrm{Al}^{3+}$ war ein Anstieg auf $80 \% \mathrm{zu}$ verzeichnen, und bei $\mathrm{pH} \mathrm{3,5}$ und $2,67 \mathrm{mg} / \mathrm{A} \mathrm{Al}^{3}+\mathrm{lag}$ die Mortalitätsrate nach 24 Stunden bei $100 \%$. 


\subsubsection{Plecoptera}

Die Plecopteren sind die artenreichste Tiergruppe in Bergbächen. Die meisten Arten sind an die hohen Fließgeschwindigkeiten, das steinige Substrat und insbesondere die niedrigen Wassertemperaturen in hohem Maße angepaßt. Viele Arten zeigen eine große Empfindlichkeit gegenüber der Gewässerversauerung (BRAUKMANN 1987).

Brachyptera seticornis: Abgesehen von den stark versauerten Probestellen S1, B1 und L1 sowie der mäßig versauerten L2 konnte die Art an allen Stellen nachgewiesen werden. An $M$ und V1 wurde Brachyptera seticornis nur in der Emergenz gefunden. In den nicht versauerten Bächen und an L3 ist die mittlere Besiedlungsdichte mit Werten zwischen einzelnen bis $27 \mathrm{lnd} . / \mathrm{m}^{2}$ gering. An SC, S3 und S2 wurden hingegen mittlere Dichten zwischen 100 und über 200 Ind. $/ \mathrm{m}^{2}$ festgestelit. Aufgrund des Entwicklungszyklus sind zwischen Mai und September keine Larven in den Bächen zu finden. Die Ergebnisse deuten darauf hin, daß die Art eine permanente starke Versauerung mit durchschnittlichen $\mathrm{pH}$ Werten unter 5 nicht toleriert, kurzfristig aber auch wesentlich höhere Wasserstoffionenkonzentrationen bis nahe $\mathrm{pH} 4$ ertragen kann.

Amphinemura: Die Gattung ist an allen Probestellen der Untersuchungen nachzuweisen, wobei die Besiedlungsdichten, die zwischen 34 und 585 Ind./ $\mathrm{m}^{2}$ liegen, keinen Zusammenhang zur Gewässerversauerung erkennen lassen. Die beiden vorkommenden Arten Amphinemura standfussi und Amphinemura sulcicollis zeigen scheinbar eine unterschiedliche Säuretoleranz. Während Amphinemura standfussi abgesehen vom Varleybach in allen anderen Gewässern vorkommt und damit auch sehr niedrige mittlere $\mathrm{pH}$-Werte von 4,2 bei einem minimalen $\mathrm{pH}$ von 3,7 toleriert, fehlt Amphinemura sulcicollis an den stark versauerten Stellen und an L2. Die Art wurde bis zu einem mittleren $\mathrm{pH}$ von 5,2 gefunden, erträgt aber auch periodische Absenkungen des $\mathrm{pH}$ auf Werte um 4,0. Amphinemura standfussi ist an $\mathrm{S1}$ im Benthos regelmäßig mit hohen Besiedlungsdichten ausschließlich in den Sommermonaten vor und bis zu Beginn der Emergenz, die sich von August bis Oktober erstreckt, zu finden (Abb. 65).

Bei anderen Untersuchungen wurden bisher beide Arten in stark versauerten Gewässern gefunden. Auch für Amphinemura sulcicollis liegen mehrere Nachweise bei sehr niedrigen pH-Werten vor (MATTHIAS 1983, STONER et al. 1984, WESTERMANN 1990). Von Amphinemura standfussi ist ebenfalls 


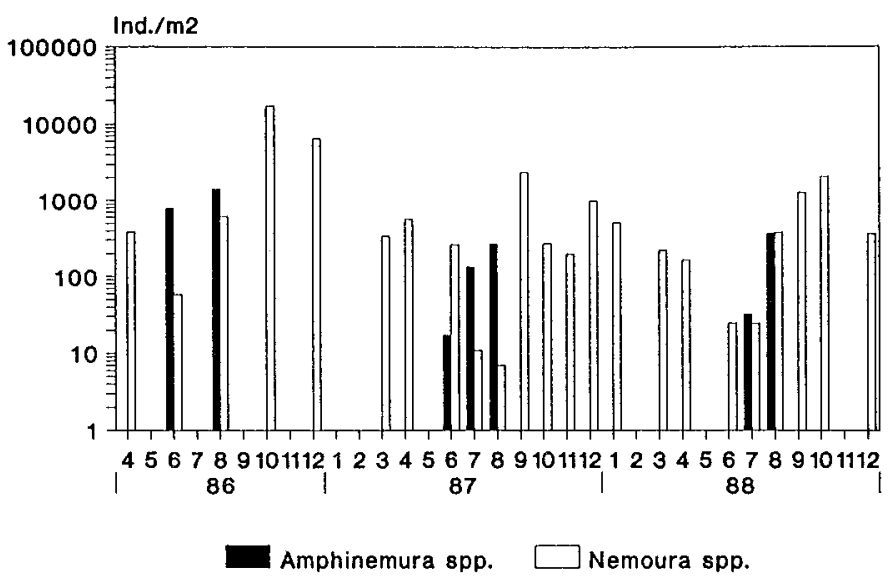

Abb. 65: Benthos-Abundanzen von Amphinemura spp. und Nemoura spp. an S1

bekannt, daß sie langfristig $\mathrm{pH}$-Werte $<5,0$ erträgt (RADDUM \& FJELLHEIM 1984, MATTHIAS 1983, STONER et al. 1984).

Nemoura spp. / Nemurella pictetii: Die Gattung Nemoura kommt an allen Probestellen vor, und Nemurella pictetii konnte nur im Varleybach nicht nachgewiesen werden. Die höchsten mittleren Benthosdichten werden an den am stärksten versauerten Probestellen mit $2013 \mathrm{Ind} . / \mathrm{m}^{2}$ an L1 und $1503 \mathrm{Ind} . / \mathrm{m}^{2}$ an S1 erreicht. An S1 stellt Nemoura cambrica den weitaus größten Teil der Individuen der Gattung. Die Besiedlungsdichten schwanken hier im Jahresverlauf sehr stark. Sie erreichen regelmäßig in den Herbstmonaten ihr Maximum, gehen den Winter über leicht zurück und zeigen mit einsetzender Emergenz einen schnellen Rückgang auf das Minimum in den Sommermonaten (Abb. 65). Mit abnehmender Versauerung sinken auch die Besiedlungsdichten der Gattung, so daß in den nicht und schwach versauerten Bächen nur noch im Mittel maximal 104 Ind. $/ \mathrm{m}^{2}$ festgestellt werden können.

Die laborexperimentellen Untersuchungen der Gattung erbrachten keine nennenswerte Mortalität bei pH 3,5 ohne und mit zusätzlicher Belastung durch Aluminium-Konzentrationen von etwa $1 \mathrm{mg} / \mathrm{l}$ (AVERMANN 1990a). Bei 


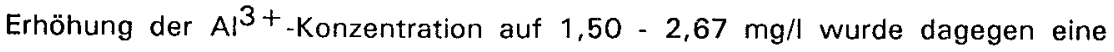
Mortalitätsrate von $43 \%$ nach 48 Stunden erreicht. Eine Differenzierung der Ergebnisse nach Arten war anhand der Larven nicht möglich.

Von den vier im Freiland vorkommenden Arten hat Nemoura cambrica die weiteste Verbreitung. Sie fehit nur an $L 1$ und toleriert damit auch die extremsten festgestellten Versauerungsbedingungen. Im Bayerischen Wald fand SCHÖLL (1987) die Art noch bei mittleren pH-Werten von 4,5. Im Kaufunger Wald und Hils kommt sie zwar auch bei pH-Werten bis 4,8 bzw. 4,2 vor, in ganzjährig versauerten Bächen aber nicht (MATTHIAS 1983, WESTERMANN 1990).

Nemoura cinerea konnte nur an fünf Stellen gefunden werden, die alle an versauerten Gewässern liegen. Ihr Vorkommen an $\mathrm{S1}$ weist darauf hin, daß sie auch sehr niedrige $\mathrm{pH}$-Werte bis 3,7 überlebt, was die Ergebnisse von MATTHIAS (1983) bestätigt.

Nemoura flexuosa wurde nur an L3 mit einem minimalem $\mathrm{pH}$ von 5,6 und an $\mathrm{V} 2$ gefangen. Die Art fehlt allgemein in quellnahen Abschnitten (KLOTZEK 1973, BLANKE 1988) und in versauerten Bereichen (HARMANEN 1980, BRAUER et al. 1990), was auch auf die im Harz untersuchten Gewässer zutrifft.

Der Nachweis von Nemoura marginata beschränkt sich auf die Alte Riefensbeek und L3, worin sich eine ähnliche Säuretoleranz wie bei Nemoura flexuosa ausdrückt. Bei anderen Untersuchungen wurde sie ausschließlich in nicht versauerten Bächen gefunden (MATTHIAS 1983, BRAUER et al. 1990, WESTERMANN 1990).

Nemurella pictetii beweist mit ihrer weiten Verbreitung ihre große Anpassungsfähigkeit an extreme Lebensbedingungen, wobei sie auch die niedrigsten $\mathrm{pH}$ Werte toleriert. An einigen der stark versauerten Probestellen kommt es dabei zu Massenentwicklungen. Die große pH-Toleranz und eine Abundanzzunahme mit der Versauerung wurden auch in anderen Untersuchungsgebieten beobachtet (MATTHIAS 1983, UBA 1987, WESTERMANN 1990).

Protonemura: Die Gattung Protonemura ist im Benthos aller Probestellen zu finden. Die höchsten mittleren Besiedlungsdichten werden mit maximal 1517 Ind. $/ \mathrm{m}^{2}$ an quellnahen Stellen der versauerten Gewässer erreicht. Über die 
Emergenz konnten 6 Arten nachgewiesen werden, die sehr unterschiedliche Säuretoleranzen zeigen.

Protonemura auberti ist die einzige Art der Gattung, die an allen Probestellen vorkommt. Die für sie beschriebene große Säuretoleranz bestätigt sich (MATTHIAS 1983, UBA 1987).

Der Fang von Protonemura hrabei an R2, R3 und S2 läßt eine gewisse Säuretoleranz dieser Art vermuten.

Protonemura intricata ist an Stellen der nicht versauerten Bäche und an B2 und L3 zu finden, was darauf hinweist, daß die Art zwar nur bei durchschnittlichen pH-Werten über 5 vorkommt, aber auch kurzzeitig niedrigere Werte erträgt. Von MATTHIAS (1983) wurde die Art ebenfalis auch in periodisch sauren Bächen bis $\mathrm{pH} 3,9$ gefunden. In anderen Untersuchungsgebieten kommt sie nur bei $\mathrm{pH}$ Werten über 6 vor (BRAUER et al. 1988, WESTERMANN 1990).

Protonemura meyeri tritt neben den nicht versauerten auch an schwach und mäßig versauerten Stellen bei einem mittleren $\mathrm{pH}$ von 5,2 und Minimum-pH von 4,3 auf. Auffällig ist das Fehlen in den quellnahen Bereichen der versauerten Gewässer. In Großbritannien wurde sie noch bei pH-Werten zwischen 3,7 und 4,5 gefunden (STONER et al. 1984), wohingegen sie in Skandinavien und Mitteleuropa nicht in stark versauerten Bereichen nachgewiesen werden konnte (HARMANEN 1980, MATTHIAS 1983, WESTERMANN 1990).

Zwei Arten ausschließlich der nicht versauerten Bäche sind Protonemura nimborum und Protonemura nitida, von denen die erste allerdings nur in der Alten Riefensbeek gefangen wurde. Bei anderen Untersuchungen wurde Protonemura nitida im neutralen bis schwach sauren Bereich gefunden (MATTHIAS 1983, BRAUER et al. 1990, WESTERMANN 1990).

Leuctra: Die Gattung Leuctra ist mit 13 Arten die artenreichste Gattung der Untersuchungsgewässer, wobei an R1 10 Arten gemeinsam vorkommen. Auch quantitativ stellt sie an allen Stellen einen großen Teil der Benthosorganismen. Die höchsten mittleren Dichten weisen $\$ 1$ mit 2252 Ind./ $\mathrm{m}^{2}$, V1 mit 1683 Ind./ $\mathrm{m}^{2}$ und $\mathrm{L} 1$ mit 1525 Ind. $/ \mathrm{m}^{2}$ auf. An allen anderen Stellen werden zwischen 500 und 1000 Ind. $/ \mathrm{m}^{2}$ erreicht. 
Leuctra nigra ist an allen 15 Probestellen zu finden. Ebenfalls an stark versauerten Stellen kommen Leuctra aurita, Leuctra pseudocingulata, Leuctra pseudosignifera und Leuctra rauscheri vor. Alle fünf Arten können daher als sehr säuretolerant angesprochen werden, da sie mittlere $\mathrm{pH}$-Werte von 4,2 und minimale Werte von 3,7 ertragen. Die große Säuretoleranz von Leuctra nigra ist schon bei zahlreichen Untersuchungen beobachtet worden, wobei die Art in den sauersten Gewässern in der Regel das Abundanzmaximum erreicht (ZWICK 1967, ZIEMANN 1975, UBA 1987, BAUER et al. 1988, BLANKE 1988, WESTERMANN 1990). Leuctra aurita wurde im Kaufunger Wald bis $\mathrm{pH} 4,8$ gefunden (MATTHIAS 1983). Leuctra pseudosignifera kommt im Bayerischen Wald bei mittleren pH-Werten von 5,7 (SCHÖLL 1987) und im Hils bis $\mathrm{pH} \mathrm{4,7}$ vor (WESTERMANN 1990).

Ebenfalls an einer stark versauerten Probestelle (S1) wurden einzelne Exemplare von Leuctra prima gefangen. Die sehr früh im Jahr schlüpfende Art quellnaher Abschnitte des Epirhithrals (ILLIES 1952, CASPERS \& STIERS 1977) konnte darüber hinaus an den beiden anderen Stellen der Großen Söse und in der Alten Riefensbeek gefunden werden. Der Nachweis der Art im gesamten Lauf beider Bäche spricht gegen ein nur quellnahes Auftreten. Aufgrund der Emergenz bereits im Februar/März ist es wahrscheinlich, daß die Art eine wesentlich weitere Verbreitung zeigt und an einer Reihe weiterer Probestellen der Nebenuntersuchungsbäche vorkommt, an denen zu diesem Zeitpunkt noch keine Fallen aufgestellt waren. Leuctra prima wird in der Literatur als vorwiegend in Gewässern mit durchschnittlichen $\mathrm{pH}$-Werten $\geq 6$ vorkommend beschrieben, wo sie nur periodische Absenkungen des $\mathrm{pH}$-Wertes auf minimal 4,8 bis 5,4 toleriert (ZIEMANN 1975, MATTHIAS 1983, BRAUER et al. 1990).

Mit Leuctra albida, Leuctra braueri, Leuctra hippopus, Leuctra inermis und Leuctra major ist eine Gruppe von fünf Arten neben den nicht versauerten Probestellen auch an ausschließlich schwach und mäßig versauerten Stellen zu finden. Von diesen Arten zeigen Leuctra albida, Leuctra braueri und Leuctra inermis mit ihrer Emergenz an $\mathrm{B} 2$ bzw. $\mathrm{S} 2$ bei einem mittleren $\mathrm{pH}$ von 5,2 und Minimum-pH um 4 die größten Säuretoleranzen. Im Kaufunger Wald kommt Leuctra albida bei noch höheren Säurebelastungen vor (MATTHIAS 1983). Leuctra braueri wurde nur an der quellnächsten Stelle der Alten Riefensbeek zahlreich gefangen. An allen anderen Stellen und insbesondere in den versauerten Bereichen bestehen die Nachweise nur aus einzelnen Tieren. Die Art gilt als stenök; sie lebt im Krenal und Hypokrenal (ILLIES 1955, DITTMAR 1955, 
RAMACHERS \& BLANKE 1987). In versauerten Gewässern wurde sie bisher nicht gefunden (ZIEMANN 1975, WESTERMANN 1990). Die im Harz weit verbreitete und an den mäßig sauren Probestellen zahlreiche Leuctra inermis kommt in anderen Gebieten überwiegend nur in Gewässern mit mittleren pHWerten $\geq 6$ vor, die nur periodisch sauer sind (MATTHIAS 1983, BRAUER et al. 1990). Im Bayerischen Wald wird sie auch bei mittleren $\mathrm{pH}$-Werten von 4,5 gefunden (SCHÖLL 1987). Die im Harz neben Leuctra major nur im schwach sauren Bereich bis minimal etwa pH 5 nachgewiesene Leuctra hippopus wurde auch bei $\mathrm{pH}$-Werten zwischen 3,7 und 4,4 gefangen IOTTO \& SVENSSON 1983, STONER et al. 1984).

Capnia vidua: Die Art stellt durch ihr teilweise recht zahlreiches Vorkommen ausschließlich in der Großen Bode/Warmen Bode eine Besonderheit dar. Beide Probestellen sind neben hohen Wasserstoffionenkonzentrationen bei einem mittleren $\mathrm{pH}$ von 4,6 an $\mathrm{B} 1$ durch relativ hohe Huminsäuregehalte geprägt, die das Entstehen toxischer Aluminium-Species teilweise verhindern können (vgl Kap. 5.1.5.10). Im Harz ist die Art auch in der Oder zu finden (GRASHOF 1972).

Setipalpia: Von den räuberischen Setipalpia-Arten tolerieren nur wenige sehr niedrige $\mathrm{pH}$-Werte. Diura bicaudata ist die einzige Art, die an allen stark versauerten Stellen vorkommt. In den versauerten Bächen ist sie mit im Mittel bis zu 20 Ind. $/ \mathrm{m}^{2}$ wesentlich zahlreicher als in den nicht versauerten. Sie ist damit säureunempfindlicher als von RADDUM \& FJELLHEIM (1984) mit pH 5,5 angegeben.

Isoperla-Larven konnten nur an $M$ und L1 nicht nachgewiesen werden. An allen versauerten Stellen bleiben die Dichten im Vergleich zu den nicht versauerten niedrig, und es wurden dort auch keine bis zur Art bestimmbaren, größeren Larven gefangen. Die Gattung ist mit im Mittel $127 \mathrm{Ind} . / \mathrm{m}^{2}$ an $\mathrm{R} 1$ am zahlreichsten. Die Bestimmungsmerkmale der älteren Larvenstadien sprechen dafür, daß es sich zumindest an dieser Stelle ausschließlich um Isoperla goertzi handelt. Die Art wurde im Bayerischen Wald nur bei pH-Werten $>5,5$ gefunden (SCHÖLL 1987) und kam im Hils an permanent versauerten Gewässern ebenfalls nicht vor (WESTERMANN 1990). Ergänzende laborexperimentelle Untersuchungen mit Isoperla-Larven erbrachten eine Mortalitätsrate von $55 \%$ bei pH 3,5 nach 48 Stunden, die durch Zugabe von $1,36 \mathrm{mg} / 1 \mathrm{Al}^{3+}$ auf $89 \%$ anstieg. Bei 2,67 $\mathrm{mg} / \mathrm{A} \mathrm{I}^{3+}$ starben alle Versuchstiere (AVERMANN 1990a). 
Eine weite Verbreitung mit teilweise recht hohen Besiedlungsdichten zeigen die beiden Chloroperliden Chloroperla tripunctata und Siphonoperla torrentium. Siphonoperla torrentium kommt auch an der stark versauerten Stelle $M$ mit einem mittleren $\mathrm{pH}$ von 5,0 und einem Minimum- $\mathrm{pH}$ von 4,2 vor. Bei anderen Untersuchungen wurde ihre relativ große Säuretoleranz ebenfalls festgestellt (STONER et al. 1984, BRAUER et al. 1990). Beide Arten erreichen ihre höchsten mittleren Besiedlungsdichten mit rund 80 bis $110 \mathrm{lnd} . / \mathrm{m}^{2}$ an SC und S2. Siphonoperla torrentium ist an R3 etwa gleich stark vertreten. Alle quellnahen Bereiche werden von beiden Arten nur schwach oder gar nicht besiedelt. Chloroperla tripunctata kann an keiner der mäßig versauerten Stellen die Entwicklung abschließen. Der minimale $\mathrm{pH}$-Wert darf daher $\mathrm{pH} 5$ wahrscheinlich nicht wesentlich unterschreiten.

Dinocras cephalotes ist eine Art, die ausschließlich in den nicht versauerten Bächen vorkommt. Sie wurde bisher bis minimal pH 5,5 gefunden (RADDUM \& FJELLHEIM 1984, PECHARSKY 1984).

Perla marginata tritt an den untersten Probestellen der beiden nicht versauerten Bäche und der Großen Söse in nur sehr geringer Dichte auf. Ob ihr eine dauerhafte Besiedfung von S3 möglich ist, konnte nicht festgestellt werden. Das Verbreitungsmuster deutet auf eine hohe Säureempfindlichkeit.

Die nur in geringer Individuenzahl vorkommende Gattung Perlodes kann nur die nicht versauerten Probestellen dauerhaft besiedeln und ihre Entwicklung abschließen. In den versauerten Gewässern sind die Larven nur in den Herbstmonaten zu finden.

\subsubsection{Coleoptera}

Nur bei wenigen Coleopteren-Arten kann aufgrund der Freilandbeobachtungen auf eine geringe Säuretoleranz geschlossen werden. Die Abundanzen zeigen jedoch bei vielen Arten deutliche Unterschiede zwischen den Probestellen der verschiedenen $\mathrm{pH}$-Gruppen. Quantitativ wird die Coleopterenfauna der untersuchten Bäche weitestgehend von einigen wenigen Arten bestimmt, zu denen die Gattung Helodes, Elmis maugetii, Esolus angustatus und Limnius perrisi gehören. Diese Arten, insbesondere die Larvenstadien, lassen sich zwar auch an 
den versauerten Stellen nachweisen, sie erreichen dort aber im Vergleich mit den nicht versauerten Probestellen nur sehr geringe Besiedlungsdichten.

Elmis sp.: Die Larven der Gattung Elmis konnten an allen nicht versauerten Stellen in mittleren Dichten zwischen 66 und $120 \mathrm{lnd} . / \mathrm{m}^{2}$ gefunden werden. In gleich hoher Dichte kommen die Larven auch an L3 vor. An allen anderen Stellen, an denen sie in den versauerten Gewässern nachgewiesen werden konnten, sind sie mit nur wenigen Individuen anzutreffen. Entsprechend beschränkt sich die Verbreitung der Imagines von Elmis maugetii auf die nicht versauerten Stellen und L3. Elmis aenea wurde mit nur wenigen Exemplaren ausschließlich in der Alten Riefensbeek gefunden. Experimentelle Untersuchungen erbrachten eine große Säuretoleranz für Elmis maugetii (MATTHIAS 1983). Auch Elmis aenea wurde wiederholt bei sehr niedrigen $\mathrm{pH}-$ Werten angetroffen (STONER et al. 1984, OTTO \& SVENSSON 1983, SCULLION \& EDWARDS 1980). Die Untersuchungsergebnisse für die Gattung im Harz lassen sich aber am ehesten in der Feststellung von WEATHERLEY \& ORMEROD (1987) wiederfinden, daß das Vorkommen positiv korreliert ist mit einer relativ hohen Gesamthärte, hohen mittleren $\mathrm{pH}$-Werten und niedrigen Aluminium-Konzentrationen.

Esolus angustatus: Wie Elmis zeigt Esolus angustatus ausschließlich in den nicht versauerten Gewässern und an $\mathrm{L} 3$ hohe Besiedlungsdichten, wobei sowohl in der Alten Riefensbeek als auch im Varleybach bachabwärts eine starke Zunahme zu verzeichnen ist. Es werden im Mittel maximal 296 bzw. 405 Ind./ $/ \mathrm{m}^{2}$ erreicht. Die relativ geringe Dichte von $27 \mathrm{Ind} . / \mathrm{m}^{2}$ an $\mathrm{R} 1$ ist eventuell auf die geringe Beschattung dieser Stelle zurückzuführen (KNIE 1977). Alle anderen versauerten Stellen weisen nur wenige Exemplare auf, darunter auch Imagines. Die Art wurde im Kaufunger Wald nur in nicht versauerten Gewässern gefunden (MATTHIAS 1983). Im Hils kam sie bei zeitweiliger Versauerung bis $\mathrm{pH} \mathrm{4,1}$ vor (WESTERMANN 1990).

Limnius perrisi: Mit mittleren Larven-Besiedlungsdichten zwischen 135 und 350 Ind./ $\mathrm{m}^{2}$ stellt Limnius perrisi die häufigste Coleopteren-Art in der Alten Riefensbeek dar. Im Varleybach ist er mit Dichten zwischen 257 und 317 Ind. $/ \mathrm{m}^{2}$ die zweithäufigste Coleopteren-Art. Vergleichbar hohe Dichten werden darüber hinaus nur an L3 erreicht. An den restlichen versauerten Stellen sind die Werte sehr niedrig, wobei sich jedoch die beiden schwach sauren Stellen SC und S3 mit leicht höheren Dichten von den anderen Stellen absetzen. Imagines konnten auch an allen Stellen gefunden werden, wo Larven vorkommen. Aus anderen 
Untersuchungen ist die recht große Säuretoleranz der Art bekannt (SCHÖLL 1987, BAUER et al. 1988). In einigen Gebieten konnte Limnius nur im neutralen bis schwach sauren Bereich gefunden werden (MATTHIAS 1983, WESTERMANN 1990).

Auf eine hohe Säureempfindlichkeit kann aufgrund der Verbreitung bei den Hydraena-Arten, Oreodytes rivalis und Deronectes sp. geschlossen werden, die ausschließlich an nicht oder nur schwach versauerten Probestellen vorkommen. Im Kaufunger Wald wurde die Gattung Hydraena nur in nicht versauerten Gewässern gefunden (MATTHIAS 1983), während sie nach BAUER et al. (1988) auch in Bächen mit periodisch absinkenden $\mathrm{pH}$-Werten bis 4,6 vorkommt. Für Oreodytes rivalis liegen Nachweise aus Gewässern mit mittleren pH-Werten um 4,0 vor (SCULLION \& EDWARDS 1980, HARRIMAN \& MORRISON 1982). Eine interessante Verbreitung zeigt Agabus guttatus, dessen Imagines ausschließlich an den beiden stark versauerten Stellen $M$ und $L 1$ gefangen wurden. Die Art des Krenals und Hypokrenals tritt auch in anderen Gebieten fast nur in den oberen Abschnitten versauerter Bäche auf (MATTHIAS 1983, WESTERMANN 1990). 


\subsection{Benthosuntersuchungen}

Unter Berücksichtigung ausschließlich der bei den Benthosuntersuchungen ermittelten Artenzahlen ergeben sich für die einzelnen Probestellen meist deutlich niedrigere Werte als mit der Emergenzerfassung zusammen, da aus der artenreichen Gruppe der Plecopteren die Filipalpia in der Regel als Larven nur bis zur Gattung bestimmbar sind. Aber auch bereits aus der Benthosuntersuchung wird die Abhängigkeit der Anzahl der vorkommenden Arten (i.S.v. Arten und höheren Taxa) vom Grad der Gewässerversauerung deutlich (Abb. 66). Für 1986, dem Jahr aus dem eine gleich große Zahl an Probenahmen für alle Stellen vorliegt, wurden an den nicht versauerten Probestellen 24 - 30 Arten, den stark versauerten 5 - 10 Arten, den mäßig versauerten 12 - 16 Arten und den schwach versauerten 15 - 21 Arten gezählt. Eine Überschneidung in der Artenzahl der einzelnen pH-Gruppen ergibt sich damit nur zwischen den mäßig und schwach versauerten Probestellen aufgrund der relativ niedrigen Artenzahl an L3.

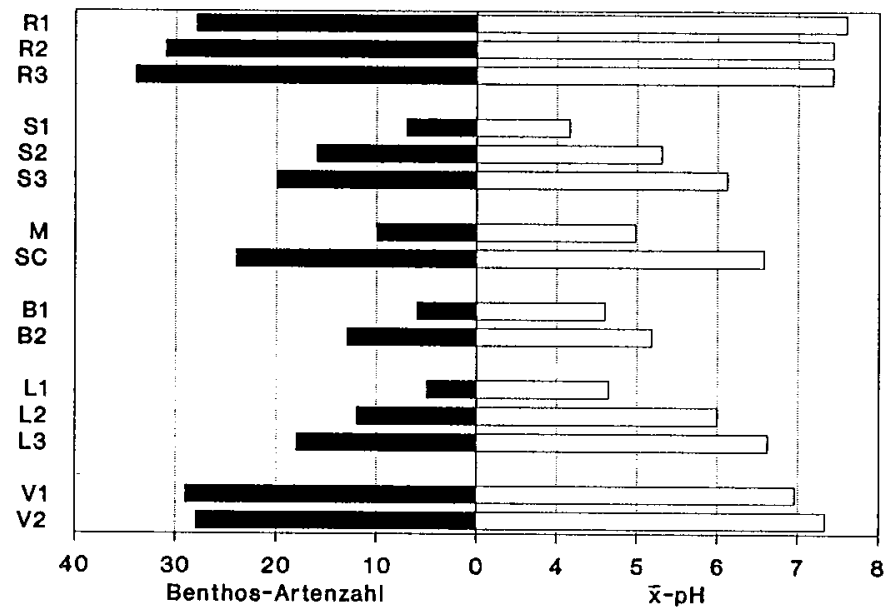

Abb. 66: Benthos-Artenzahlen im Vergleich mit den mittleren $\mathrm{pH}-$ Werten der Probestellen 
Veränderungen im Artenspektrum zwischen den einzelnen Jahren treten unregelmäßig und im Ausmaß unabhängig vom Versauerungsgrad auf. Sie betreffen ausschließlich Arten mit niedrigen Abundanzen und sind in erster Linie auf unregelmäßige Besiedlungen durch einige Coleopteren-Arten sowie in geringem Umfang auf nur in einzelnen Jahren nachgewiesene seltene Ephemeropterenund Plecopteren-Arten zurückzuführen.

\subsubsection{Benthos der Hauptuntersuchungsbäche}

\subsubsection{Besiedlungsdichten}

Im Rahmen der intensiven Untersuchungen an den Probestellen von Alter Riefensbeek und Großer Söse wurden an jeder Stelle 23 Benthosbeprobungen zwischen April 1986 und Dezember 1988 durchgeführt. Die im Mittel der Probenahmen weitaus höchste Besiedlungsdichte konnte dabei für die am stärksten versauerte Probestelle S1 ermittelt werden (Abb. 67). S2 und, bei weiterem Rückgang, S3 liegen dagegen mit ihren Abundanzen deutlich unter den Zahlen der Probestellen der Alten Riefensbeek, in der ebenfalls mit R1 die quellnächste Probestelle die höchste Besiedlungsdichte des Gewässers aufweist. Zwischen

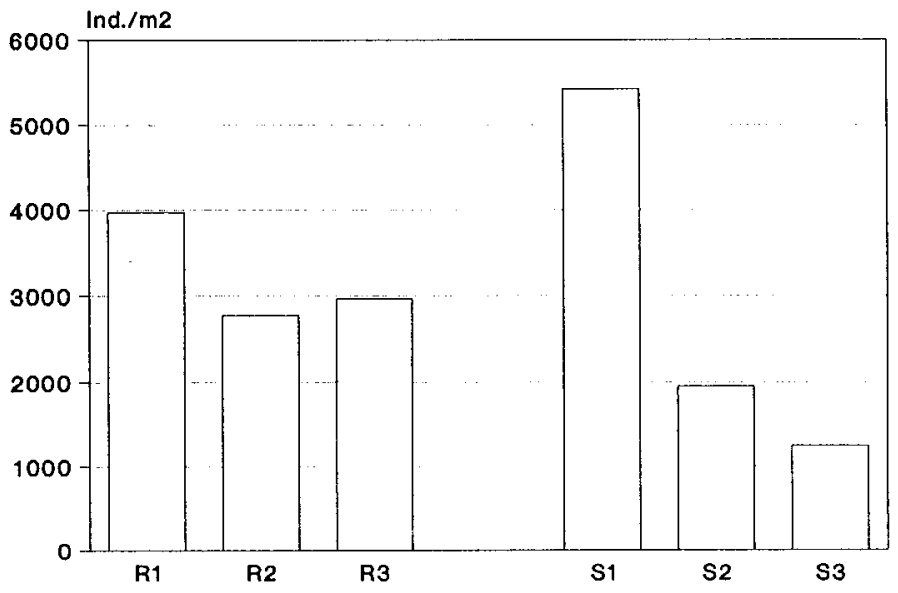

Abb. 67: Mittlere Benthosbesiedlungsdichten der Hauptuntersuchungsstellen 
R2 und R3 ergibt sich nur ein geringer Unterschied. Insgesamt erreicht die Große Söse zwar nur $89 \%$ der Benthos-Abundanz der Alten Riefensbeek, \$1 aber $136 \%$ von R1.

Betrachtet man die Einzeljahre, so wird deutlich, daß sich die hohe Besiedlungsdichte an S1 zum einen aus einer außergewöhnlich hohen Dichte im Herbst 1986 erklärt, zum anderen die Besiedlungsdichten, abgesehen von Minima in den Sommermonaten, beinahe zu allen Probenahmeterminen deutlich über denen der mittleren und unteren Probestelle der Großen Söse liegen (Abb. 68 - 70). Im Herbst 1986 weisen auch S2 und S3 ein absolutes Abundanzmaximum auf. Der Vergleich mit der Emergenzerfassung in diesem Jahr zeigt für die Monate ab Juli keine ungewöhnlich hohen Zahlen (vgl. Abb. 98). Das Maximum der Emergenz dürfte aber, wie in den folgenden Jahren, bereits im Juni gelegen haben und damit nicht erfaßt worden sein. In allen drei Jahren nehmen die Benthosbesiedlungsdichten in der Großen Söse bachabwärts stark ab. \$3 hat mit alljährlichen Maxima in den Herbstmonaten und Minima im Sommer den regelmäßigsten Jahresgang.

In der Alten Riefensbeek sind unter anderem aufgrund der größeren Zahl emergenzunabhängiger Arten die Besiedlungsdichten insgesamt etwas ausgeglichener als in der Großen Söse. R1 weist konstant in jedem Jahr die höchsten Dichten auf (Abb. 71 - 73). 1986 konnte auch hier eine ungewöhnlich hohe mittlere Jahresabundanz festgestellt werden, die an dieser Stelle vor allem auf sehr hohen Abundanzen von Gammarus pulex in den Sommermonaten beruhte. Für 1987 ergeben sich für alle Probestellen der Hauptuntersuchungsbäche wesentlich niedrigere Werte als 1986 (Abb. 74). S1 bleibt die Stelle mit der weitaus höchsten Dichte aber wesentlich geringerer Differenz zu den anderen Stellen als im Vorjahr. 1988 liegen alle Stellen der Alten Riefensbeek, mit der höchsten Dichte an R1, über den Probestellen der Großen Söse. In der Großen Söse wird aber auch in diesem Jahr die Abundanzabnahme von S1 nach \$3 sehr deutlich. S1 ist 1988 die einzige Stelle, die im Vergleich zu 1987 eine Abnahme statt einer deutlichen Zunahme der Besiedlungsdichten zeigt.

Unter Berücksichtigung der von RÜDDENKLAU (1989) angegebenen maximalen Trichopteren-Abundanzen, die sich für einen direkten Vergleich allerdings nicht eignen, sind keine wesentlichen Veränderungen im Bild der Besiedlungsdichten zu erwarten. Die Trichopteren tragen jedoch dazu bei, daß die Unterschiede zwischen den Probestellen der Alten Riefensbeek und S1 geringer und die zu S2 
und S3 größer werden müßten. Die Ordnung zeigt in der Alten Riefensbeek einen stark ausgeprägten Rückgang der maximalen Abundanzen bachabwärts von 2370 auf $890 \mathrm{Ind} . / \mathrm{m}^{2}$. Die dazu vergleichsweise sehr niedrigen maximalen Abundanzen in der Großen Söse mit $110-180 \mathrm{lnd} . / \mathrm{m}^{2}$ weisen ebenfalls eine Abnahme von $\$ 1$ nach $\$ 3$ auf.

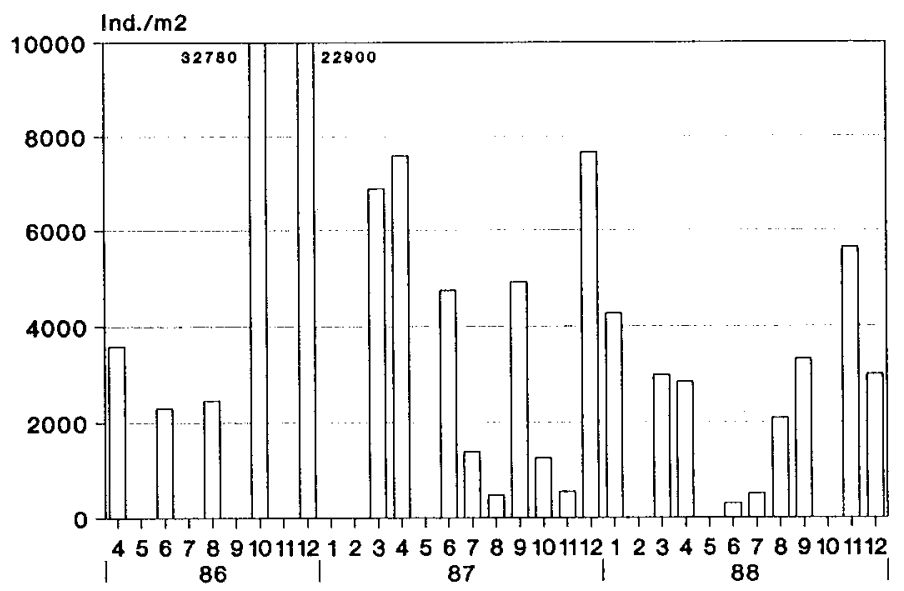

Abb. 68: Benthosbesiedlungsdichten an $\$ 1$ 


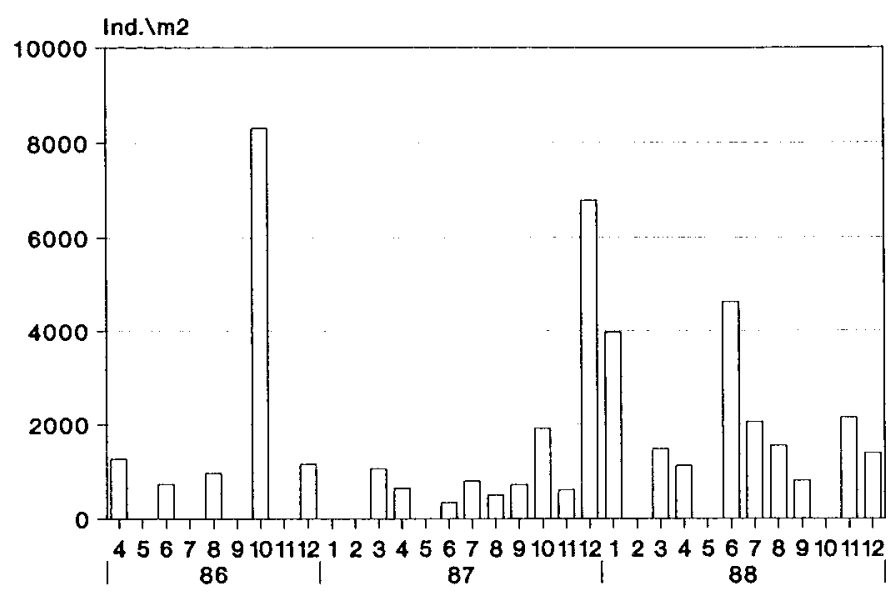

Abb. 69: Benthosbesiedlungsdichten an $\mathrm{S} 2$

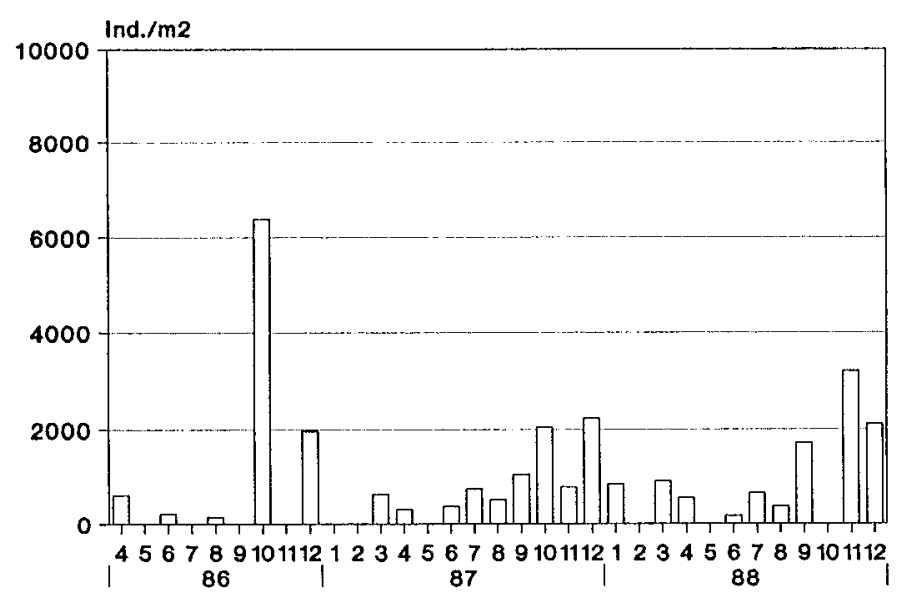

Abb. 70: Benthosbesiedlungsdichten an S3 


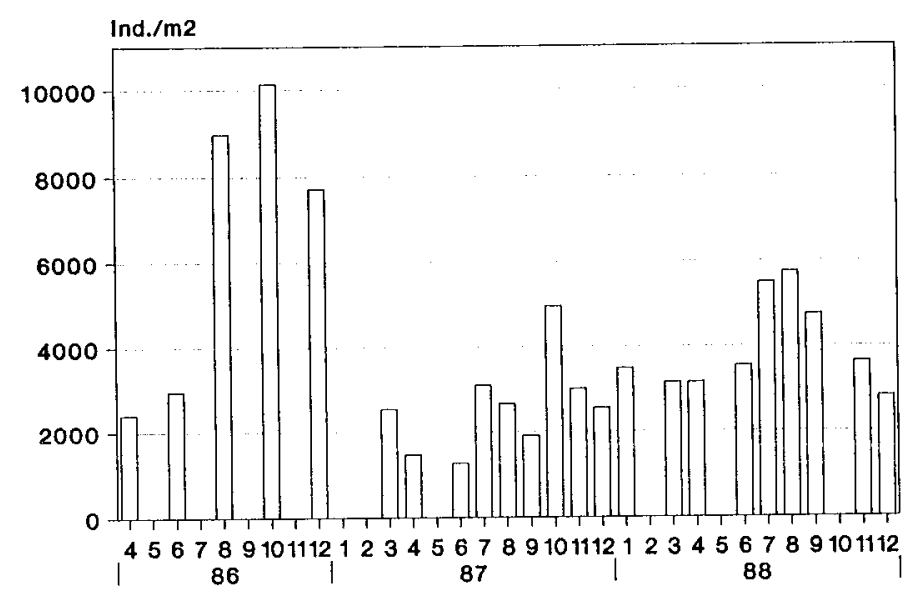

Abb. 71: Benthosbesiedlungsdichten an R1

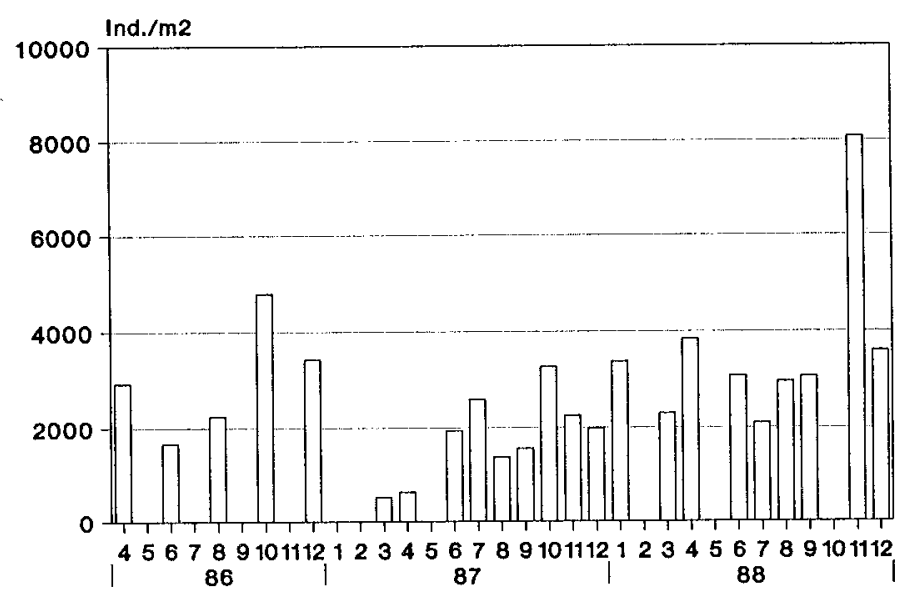

Abb. 72: Benthosbesiedlungsdichten an R2 


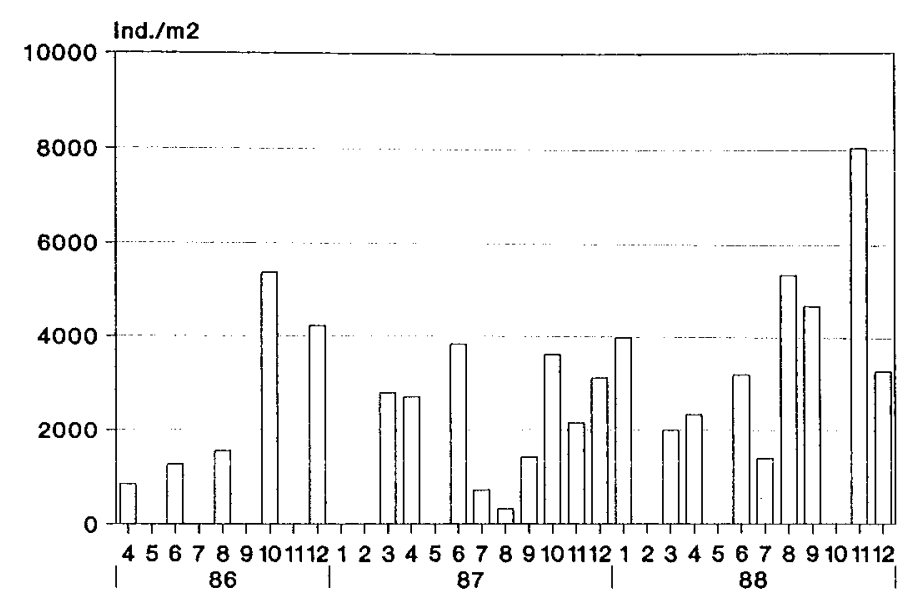

Abb. 73: Benthosbesiedlungsdichten an R3

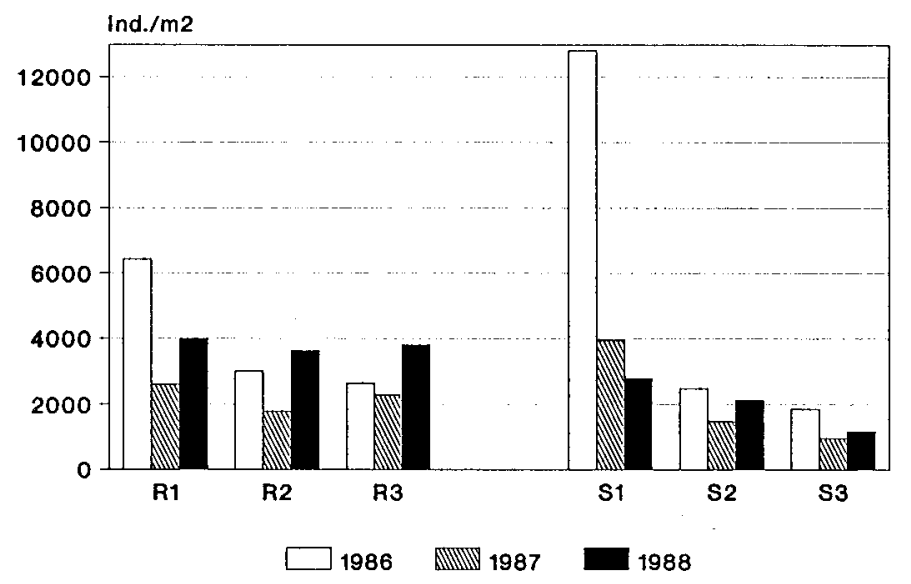

Abb. 74: Mittlere Benthosbesiedlungsdichten der Hauptuntersuchungsstellen in den Einzeljahren 


\subsubsection{Dominanzstrukturen}

Im Vergleich der beiden Bäche stellen in der Alten Riefensbeek die Gattungen Baetis und Leuctra sowie mit bereits wesentlich geringerer Dominanz Gammarus pulex die eudominanten Arten (Tab. 17). Die dominanten Arten sind Limnius perrisi, Amphinemura spp. und Esolus angustatus. In der Großen Söse gehören mit Leuctra spp., Protonemura spp. und Nemoura spp. ausschließlich Plecopteren zu den eudominanten Arten. Als dominante Art kommt die nur an S2 und S3 lebende Ephemeropteren-Gattung Baetis hinzu. Eudominante und dominante Arten haben in der Alten Riefensbeek einen Dominanzanteil von $80 \%$, während sie in der Großen Söse $90 \%$ erreichen.

Betrachtet man die Dominanzstruktur der einzelnen Probestellen, werden größere Unterschiede innerhalb der Gewässer deutlich. An allen drei Probestellen der Alten Riefensbeek stellt Baetis als wichtigste Ephemeropteren-Gattung rund ein Viertel der Gesamtbesiedlung (Abb. 75 - 77). Baetis ist damit an R1 und R2 die Art mit der höchsten Dominanz. An R3 wird diese Rolle von Leuctra übernommen, die über den Gesamtuntersuchungszeitraum betrachtet an R1 nach Gammarus pulex die dritt- und an R2 die zweithäufigste Art ist. Während an $\mathrm{R} 1$ und $\mathrm{R} 2$ noch von relativ ausgeglichenen Dominanzstrukturen gesprochen werden kann, haben an R3 die drei eudominanten Arten zusammen bereits einen Dominanzanteil von $71,3 \%$.

Die Dominanzstruktur von R3 ähnelt dadurch den Verhältnissen an S3 und S2 (Abb. 80, 79). An diesen mäßig bzw. schwach versauerten Stellen der Großen Söse erreicht Baetis jedoch nur rund $16 \%$ der Gesamtdominanz, wohingegen Leuctra an S3 mit einem Anteil von über $50 \%$ für eine sehr unausgeglichene Dominanzstruktur verantwortlich ist. Im Gegensatz zur Alten Riefensbeek finden sich unter den dominanten Arten an keiner Stelle Coleopteren. An S1 wird die Dominanzstruktur durch drei Plecopteren-Gattungen bestimmt, die 97,3\% der Dominanz stellen, ohne daß eine der Gattungen aber einen unausgewogen hohen Anteil hat (Abb. 78).

Aus den Ergebnissen der Einzeljahre lassen sich Trends in der Entwicklung der Biozönosen und der Stabilität der Dominanzstrukturen ablesen (Abb. 75 - 80). Die unter dem Gesichtspunkt der Gewässerversauerung besonders interessante Entwicklung der Gattung Baetis zeigt an allen Probestellen der Alten Riefensbeek einen maximalen Dominanzanteil der Gattung im Jahr 1987 an. An R1 und R2 
Tab. 17: Benthos-Dominanzstrukturen der beiden Hauptuntersuchungsbäche

\begin{tabular}{|c|c|c|c|c|c|c|}
\hline \multirow[b]{2}{*}{1.} & \multirow{2}{*}{$\frac{\text { Art }}{\text { Baetis sp. }}$} & \multicolumn{2}{|c|}{$\begin{array}{l}\text { Alte Riefensbeek } \\
\text { Präsenz Dominanz }\end{array}$} & \multicolumn{2}{|c|}{$\begin{array}{l}\text { Grosse Söse } \\
\text { Präsenz Dominanz }\end{array}$} & \multirow[b]{2}{*}{4.} \\
\hline & & 100 & 26,79 & 67 & 5,98 & \\
\hline 2. & Leuctra sp. & 100 & 23,41 & 100 & 41,26 & 1. \\
\hline 3. & Gammarus pulex LINNAEUS & 100 & 10,01 & 0 & & \\
\hline 4. & Limnius sp. & 100 & 7,77 & 100 & 0,13 & 12. \\
\hline 5. & Amphinemura sp. & 100 & 5,61 & 100 & 2,41 & 6. \\
\hline 6. & Esolus sp. & 100 & 5,46 & 100 & 0,09 & 14. \\
\hline 7. & Rhithrogena sp. & 100 & 3,48 & 33 & 0,10 & 13. \\
\hline 8. & Protonemura sp. & 100 & 2,34 & 100 & 21,67 & 2. \\
\hline 9. & Elmis sp. & 100 & 2,26 & 100 & 0,04 & 18. \\
\hline 10. & Ecdyomurus sp. & 100 & 2,01 & 67 & 0,04 & 19. \\
\hline 11. & Isoperla sp. & 100 & 1,73 & 100 & 0,22 & 10. \\
\hline 12. & Habroleptoides modesta HAGEN & 100 & 1,40 & 0 & & \\
\hline 13. & Siphonoperla torrentium (PICIET) & 100 & 1,29 & 67 & 2,40 & 7. \\
\hline 14. & Helodes sp. & 100 & 0,94 & 100 & 0,05 & 17. \\
\hline 15. & Ancylus fluviatilis MUELER & 100 & 0,75 & 0 & & \\
\hline 16. & Epeorus sylvicola PICTET & 100 & 0,66 & 67 & 0,06 & 16. \\
\hline 17. & Esolus angustatus (P. MELLER) & 100 & 0,56 & 100 & 0,02 & 21. \\
\hline 18. & Nemoura sp. & 100 & 0,56 & 100 & 21,11 & 3. \\
\hline 19. & Brachyptera seticomis (KLAPALEK) & 100 & 0,45 & 67 & 3,10 & 5. \\
\hline 20. & Ephemerella ignita PODA & 100 & 0,43 & 0 & & \\
\hline 21. & Ephemerella mucronata BENGISSON & 100 & 0,42 & 0 & & \\
\hline 22. & Limnius perrisi (DUFOUR) & 100 & 0,42 & 100 & 0,03 & 20. \\
\hline 23. & Elmis mugetii LATREIIE & 100 & 0,27 & 0 & & \\
\hline 24. & Habrophlebia lauta EATON & 100 & 0,26 & 0 & & \\
\hline 25. & Perlodes sp. & 100 & 0,17 & 33 & 0,02 & 22. \\
\hline 26. & Dinocras cephalotes (CARTIS) & 100 & 0,11 & 0 & & \\
\hline 27. & Niphargus sp. & 100 & 0,07 & 67 & 0,00 & 11. \\
\hline 28. & Hydraena sp. & 100 & 0,07 & 33 & 0,01 & 24. \\
\hline 29. & Elmis aenea (P. MJELIER) & 100 & 0,04 & 0 & & \\
\hline 30. & Chloroperla tripunctata (SOOPOLI) & 67 & 0,04 & 67 & 0,73 & 8. \\
\hline 31. & Hydraena palustris ER. & 100 & 0,04 & 33 & 0,00 & 25. \\
\hline 32. & Deronectes sp. & 100 & 0,03 & 0 & & \\
\hline 33. & Oreodytes rivalis (GYUENIHAL) & 67 & 0,03 & 0 & & \\
\hline 34. & Hydroporus sp. & 67 & 0,02 & 0 & & \\
\hline 35. & Oreodytes sp. & 100 & 0,02 & 33 & 0,00 & 28. \\
\hline 36. & Pisidium sp. & 100 & 0,02 & 0 & & \\
\hline 37. & Hydraena belgica D'OROH. & 67 & 0,02 & 0 & & \\
\hline 38. & Perla marginata (PANZER) & 33 & 0,01 & 33 & $0, \infty$ & 26. \\
\hline 39. & Diura bicaudata (LINNAEUS) & 100 & 0,01 & 100 & 0,22 & 9. \\
\hline 40. & Agabus sp. & 67 & 0,01 & 100 & 0,08 & 15. \\
\hline 41. & Haliplus sp. & 67 & $0, \infty$ & 0 & & \\
\hline 42. & Hydraena dentipes GERM. & 67 & 0,00 & 0 & & \\
\hline \multirow[t]{3}{*}{43.} & Anacaena globulus (PAYKULL) & 33 & $0, \infty$ & 0 & & \\
\hline & Siphlomurus aestivalis EATON & 0 & & 33 & 0,01 & 23. \\
\hline & Hydroporus nigrita (FARRICTUS) & 0 & & 33 & $0, \infty$ & 27. \\
\hline
\end{tabular}


ist sie durchgehend die Art mit der höchsten Abundanz, an R3 nur 1986. Ein Rückgang der Dominanz ist im gesamten Bach für Gammarus pulex über die drei Untersuchungsjahre zu verzeichnen. Leuctra hat dagegen allgemein einen zunehmenden Trend. Die Coleopteren unterliegen an allen Stellen größeren Schwankungen, von denen Esolus angustatus stärker betroffen ist als Limnius perrisi. In der Großen Söse zeigt die Baetis-Dominanz sehr starke Schwankungen von Jahr zu Jahr. An S3 werden die höchsten Dominanzen 1986 und 1987 erreicht, an S2 hingegen 1988 nach nur geringen Anteilen in den beiden vorausgegangenen Jahren. S2 übertrifft dabei die Werte der Stellen der Alten Riefensbeek. Den permanent höchsten Dominanzanteil mit größeren Schwankungen von Jahr zu Jahr hat an S2 und S3 Leuctra. Alle anderen dominanten Arten unterliegen z.T. großen Schwankungen. An $\$ 1$ haben die drei eudominanten Gattungen immer einen Dominanzanteil von über $96 \%$ mit allerdings stark wechselnden Anteilen der einzelnen Arten, so daß 1986 Nemoura, 1987 Leuctra und 1988 Protonemura die Gattung mit der höchsten Abundanz ist. Die Dominanzstruktur an S1 ist damit eine der instabilsten der sechs Probestellen.

Die Jahresgänge der Dominanzen sind wie die Gesamtbesiedlungsdichten in hohem Maße von den Entwicklungsgängen der eudominanten Arten abhängig. Da an allen Stellen Arten mit sehr hohen Dominanzanteilen vorkommen, zeichnen die Abundanzkurven zu einem großen Teil die Besiedlungsdichten dieser Arten nach (Abb. 68 - 73).

Für die extrem hohen Dichten an S1 im Herbst 1986 sind insbesondere Nemoura und Leuctra verantwortlich. Bis zum März 1987 findet jedoch eine Verschiebung statt, bei der Protonemura den höchsten Dominanzanteil gewinnt und Leuctra sowie insbesondere Nemoura stark zurückgehen. Zum Herbst 1987 und 1988 sind ebenfalls wieder Nemoura und Leuctra die Arten mit der höchsten Dominanz. Das Abundanzmaximum an S2 im Herbst 1986 ist wie an S1 auf Leuctra und Nemoura zurückzuführen, zu denen an dieser Stelle noch Brachyptera seticornis tritt. Zur Jahreswende 1987/88 dominieren dagegen Protonemura, Leuctra und Brachyptera seticornis. Nachdem Baetis 1986 bereits in den Sommermonaten sehr zahlreich und $1987 \mathrm{im}$ Sommer nur schwach vertreten war, ist das Abundanzmaximum im Sommer 1988 weitestgehend allein auf diese Gattung zurückzuführen. An S3 gehört Baetis hingegen in jedem Jahr zu den dominanten Sommer- und Herbstarten. In den Winter- und Frühahresmonaten hat sie keine Bedeutung. Die Wintermaxima werden vor allem von Leuctra gebildet. 
Die Probestelien der Alten Riefensbeek zeigen entsprechend den zumindest an R1 und R2 konstanteren Dominanzstrukturen eine relativ große Konstanz in der Zusammensetzung der Abundanzmaxima. Die in jedem Jahr an R3 im Herbst auftretenden hohen Abundanzen sind hier wie auch an R2 vor allem auf Baetis und Leuctra zurückzuführen. Die höheren Dichten in den Sommermonaten werden an beiden Stellen von Esolus angustatus und Leuctra ausgelöst. Baetis kann auch in der Alten Riefensbeek vereinzelt in den Sommermonaten eine höhere Abundanz erreichen, was vor allem an R1 zu beobachten ist, wobei gleichzeitig Gammarus pulex verstärkt auftritt. An allen drei Stellen bestimmt Baetis im Gegensatz zur Großen Söse den Abundanzverlauf auch in den Wintermonaten sehr wesentlich.

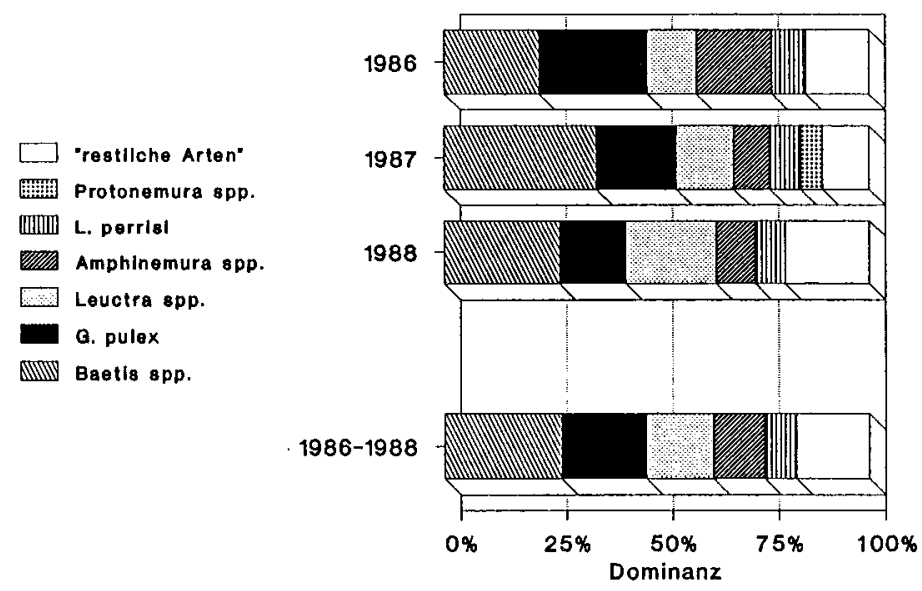

Abb. 75: Eudominante und dominante Arten an R1 


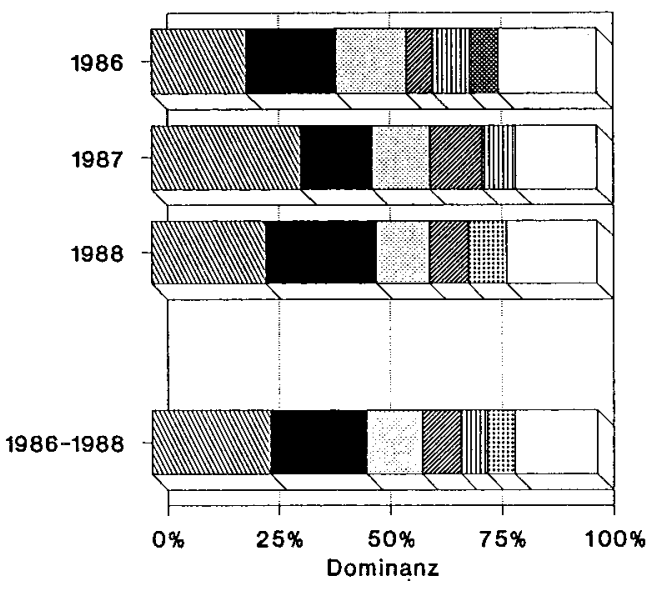

Abb. 76: Eudominante und dominante Arten an R2

$\square$ "restllche Arten"

WIII L. perris!

E. angustatus

Baetis spp.

MilW Leuctra spp.

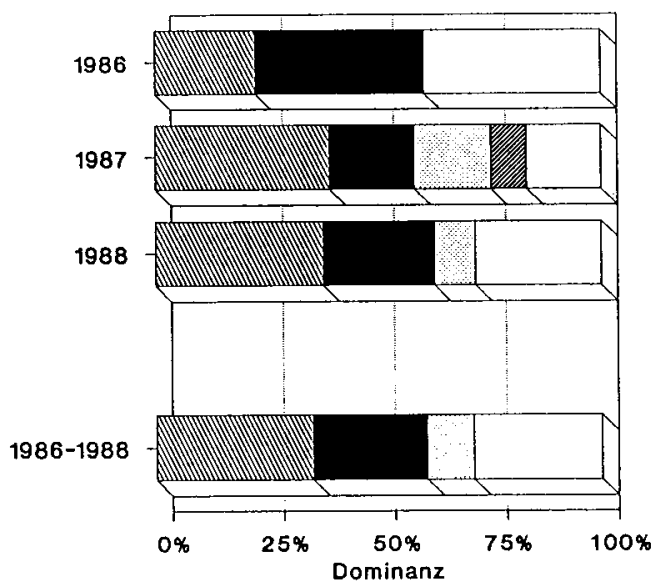

Abb. 77: Eudominante und dominante Arten an R3 
[.] "restliche Arten"

— Nemoura spp.

Protonemura spp.

MW. Leuctra spp.

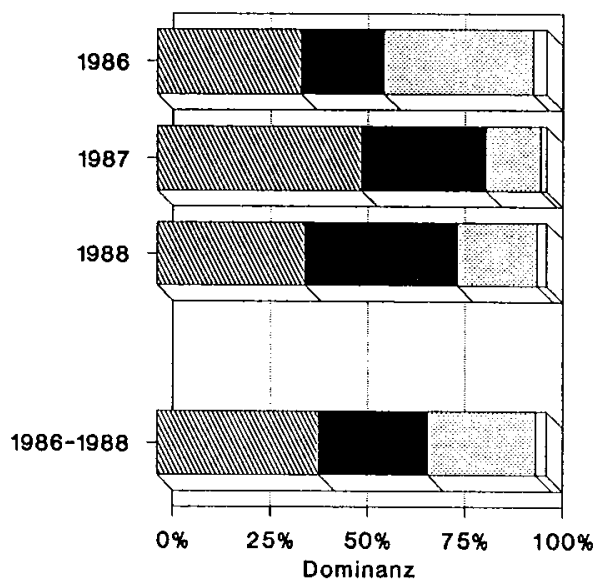

Abb. 78: Eudominante und dominante Arten an S1
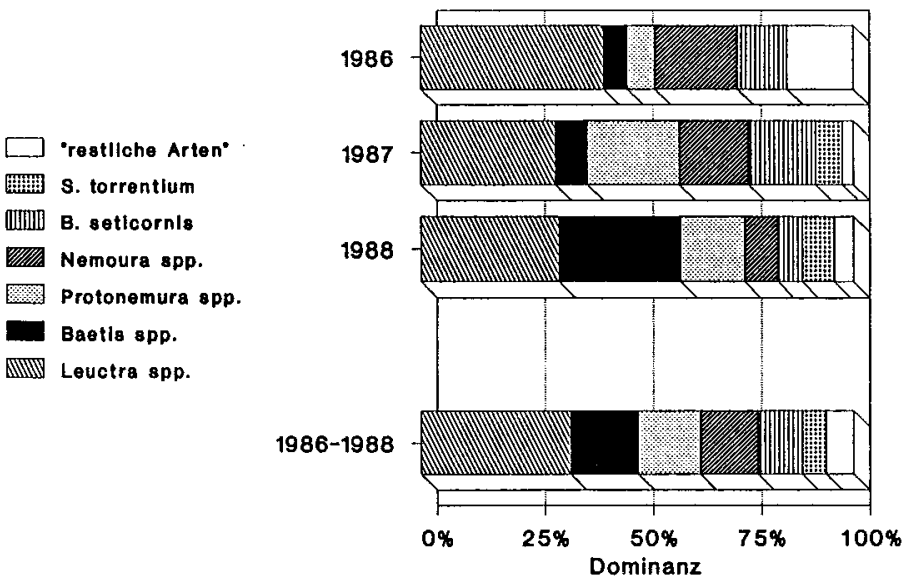

Abb. 79: Eudominante und dominante Arten an S2 

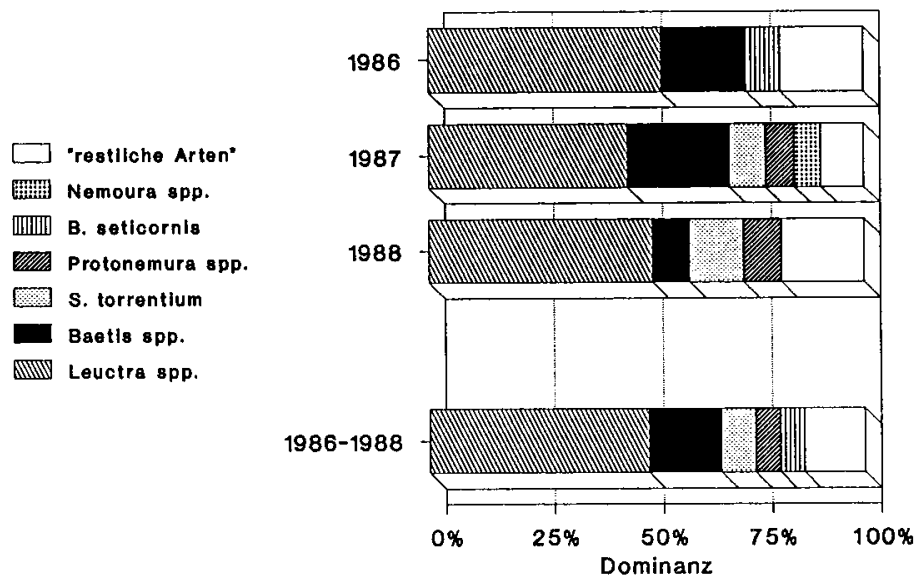

Abb. 80: Eudominante und dominante Arten an $\$ 3$

\subsubsection{Zusammenfassender Vergleich der Probestellen}

Zusammenfassend kann für die Probestellen an Alter Riefensbeek und Großer Söse festgehalten werden, daß in beiden Bächen die quellnächsten Stellen die höchsten Besiedlungsdichten aufweisen, wobei die Dichten an der stark versauerten Stelle S1, über den Zeitraum von drei Jahren betrachtet, höher sind als an R1. Die Unterschiede zwischen R2 und R3 sind gering, wohingegen von S2 nach S3 eine weitere Verringerung der Besledlungsdichten zu verzeichnen ist. Beide Stellen liegen deutlich unter den Werten des nicht versauerten Gewässers. 1988 ergibt sich auch für $\mathrm{S} 1$ eine niedrigere Dichte als für alle Probestellen der Alten Riefensbeek, wobei der Gradient von S1 nach S3 aber bestehen bleibt. Entwicklungsbedingte Abundanzmaxima sind in jedem Jahr vor allem im Sommer und Herbst zu verzeichnen. Als wichtigste Ephemeropteren-Gattung führt Baetis dabei in der Großen Söse an S2 und S3 regelmäßig zu Abundanzmaxima in den Sommermonaten. In der Alten Riefensbeek treten die durch Baetis bedingten Maxima dagegen vor allem im Herbst und Winter auf. Besiedlungsdichten und Dominanzstrukturen der Probestellen der Alten Riefensbeek 


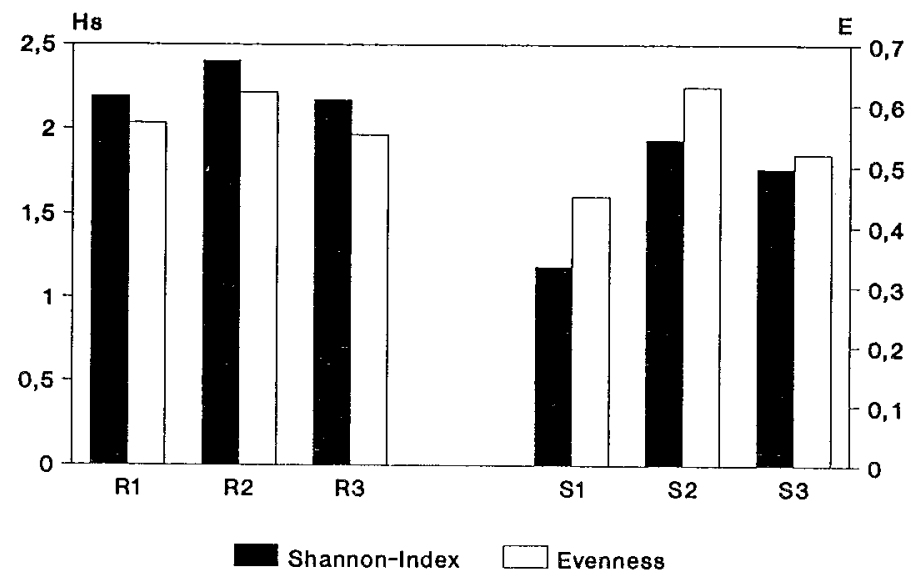

Abb. 81: Benthos-Diversitätsindices und Evenness der Probestellen der Hauptuntersuchungsbäche

sind insgesamt ausgeglichener als die der Großen Söse. Aber auch in dem nicht versauerten Gewässer können einige eudominante Arten sehr hohe Dominanzanteile erreichen, was insbesondere an der quellfernsten Probestelle festzustellen ist.

Hierauf und auf die wesentlich höheren Artenzahlen sind die besseren Werte des SHANNON-Index für die Alte Riefensbeek zurückzuführen (Abb. 81). Während in dem nicht versauerten Gewässer alle Werte über 2,15 mit einem Maximum an R2 liegen, errechnen sich für die Große Söse Werte unter 1,95 mit dem Maximum ebenfalis an der mittleren Probestelle. An beiden mittleren Stellen erreicht auch die Evenness ihr Maximum. Den niedrigsten Wert für Diversität und Evenness hat die stark versauerte Stelle S1. Die Evenness der restlichen Probestellen liegt relativ dicht beieinander. $\mathrm{Zu}$ berücksichtigen ist bei diesen Benthos-Diversitätsberechnungen allerdings, daß die Plecopteren zum größten Teil nur als Gattungen in die Berechnungen eingehen, hinter denen sich eine unterschiedliche Zahl an Arten versteckt, so daß die Emergenzerfassungen für diese Ordnung ein wesentlich zuverlässigeres Zahlenmaterial liefern können und damit auch größere Unterschiede zwischen den Probestelien zu erwarten sind. 


\subsubsection{Benthos der Haupt- und Nebenuntersuchungsbäche im Vergleich}

Anhand der Ergebnisse der 1986 und 1987 erfolgten achtmaligen Beprobung aller 15 Stellen der Haupt- und Nebenuntersuchungsbäche ist eine Überprüfung der dargestellten Ergebnisse im Hinblick auf allgemeine auf die Gewässerversauerung zurückzuführende Aussagen möglich. Aus der Zusammenstellung der mittleren Besiedlungsdichten wird deutlich, daß die beiden permanent stark sauren Probestellen S1 und L1 deutlich höhere Dichten als die jeweils weiter bachabwärts gelegenen Stellen desselben Gewässers aufweisen (Abb. 82). Die beiden stark versauerten Stellen mit großen $\mathrm{pH}$-Schwankungen $\mathrm{M}$ und $\mathrm{B} 1$ zeigen hingegen nur eine geringfügig bzw. weitgehend identische Besiedlungsdichte im Vergleich mit den unterhalb gelegenen Stellen SC bzw. B2. Alle mäßig und schwach versauerten Probestellen (B2, S2, L2 bzw. S3, SC, L3) weisen auf niedrigem Niveau relativ dicht zusammenliegende Gesamtabundanzen auf. Die Probestellen der beiden nicht versauerten Bäche liegen mit ihren Besiedlungsdichten über diesen Werten, wobei R1, V1 und V2 die nach S1 insgesamt höchsten Dichten haben. Dies läßt sich auch aus den Daten der einzelnen Untersuchungsjahre ablesen (Abb. 83). Dabei ist jedoch die unterschiedliche Zahl der gemeinsamen Probenahmen der beiden Jahre (5 bzw. 3) zu berücksichtigen. S1 kommt 1987 nur auf mit R1 und V2 vergleichbare Werte.

Die festgestellten maximalen Trichopteren-Abundanzen, die sich für direkte Vergleiche mit den mittleren Abundanzen der anderen Tiergruppen nicht eignen, haben die höchsten Werte an den nicht versauerten Probestellen, wobei nur B2 R3 übertrifft (RÜDDENKLAU 1989). Abgesehen von B2 sind die maximalen Abundanzen der versauerten Gewässer dagegen deutlich niedriger. Die sich zwischen den Probestellen ergebenden Unterschiede sind relativ groß. Wesentliche Auswirkungen auf das Bild der Gesamtabundanz sind auch bei Einbeziehung der Trichopteren nicht zu erwarten.

Aus den Dominanzstrukturen sind eine Reihe gemeinsamer Charakteristika für die verschiedenen Versauerungsgrade ablesbar. Im Varleybach gehören wie in der Alten Riefensbeek Baetis und Leuctra zu den eudominanten Arten (Abb. 84). Der Baetis-Anteil ist hier jedoch etwas niedriger als in der Alten Riefensbeek. Eine Besonderheit ist im Varleybach der hohe Anteil von Amphinemura, der sonst nur an R1 zu beobachten ist. Unter den dominanten Arten sind an allen Stellen Coleopteren zu finden. 
Alle stark versauerten Probestellen zeigen sehr unausgeglichene Dominanzstrukturen. An M treten wie an $\$ 1$ Leuctra, Protonemura und Nemoura als eudominante Arten mit einer Leuctra-Dominanz von über $54 \%$ auf (Abb. 85). Auch an L1 und B1 haben einzelne Arten sehr hohe Dominanzen (Abb. 86, 87). Neben Leuctra und Nemoura findet sich als Besonderheit der Probestellen an Großer Bode und Warmer Bode unter den eudominanten Arten Amphinemura. Die mäßig und die schwach versauerten Probestellen zeigen dagegen wesentlich ausgeglichenere Dominanzstrukturen. Außer an SC, wo Baetis die höchste Dominanz hat, ist an den restlichen Stellen Leuctra die Art mit der höchsten Abundanz.

Die Berechnung des SHANNON-Index ergibt die höchsten Werte für die nicht versauerten Probestellen und SC (Abb. 88). Die niedrigsten Werte weisen die sauersten Stellen in der Reihenfolge L1, B1, S1, M auf. Es folgen die mäßig und schwach versauerten Stellen B2, S3, L2, L3 und S2. Für alle diese Probestellen liegt der Diversitätsindex maximal bei 1,90. Einen Index über 2,10 haben alle Stellen der nicht versauerten Bäche sowie als einzige schwach versauerte Probestelle SC. Bei der Evenness sind die stark versauerten und die nicht versauerten Stelle hingegen nicht klar voneinander getrennt. Die niedrigsten Werte ergeben sich aber auch hier an L1 und $M$ für zwei stark versauerte Probestellen.

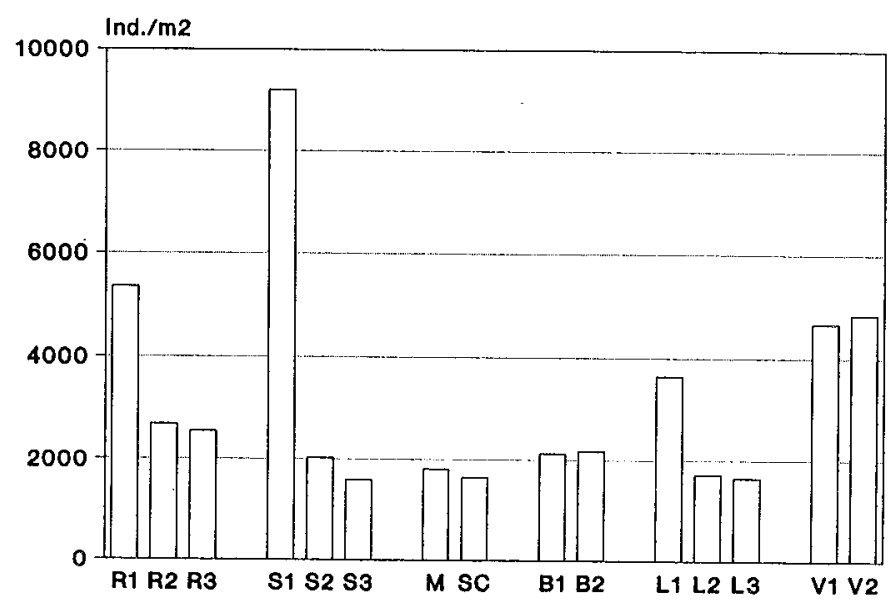

Abb. 82: Mittlere Benthosbesiedlungsdichten der gemeinsamen Probenahmen an Haupt- und Nebenuntersuchungsbächen 


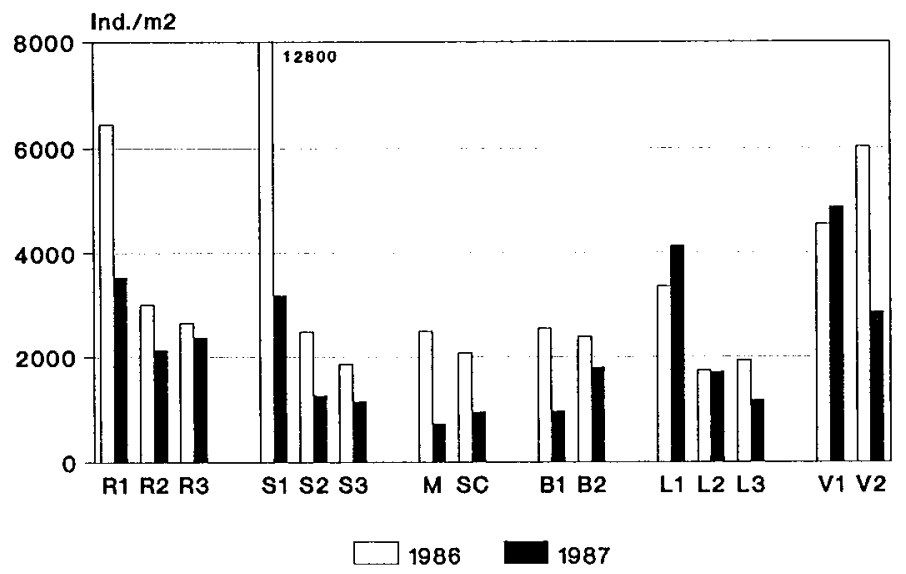

Abb. 83: Mittlere Benthosbesiedlungsdichten der gemeinsamen Probenahmen in den Einzeljahren

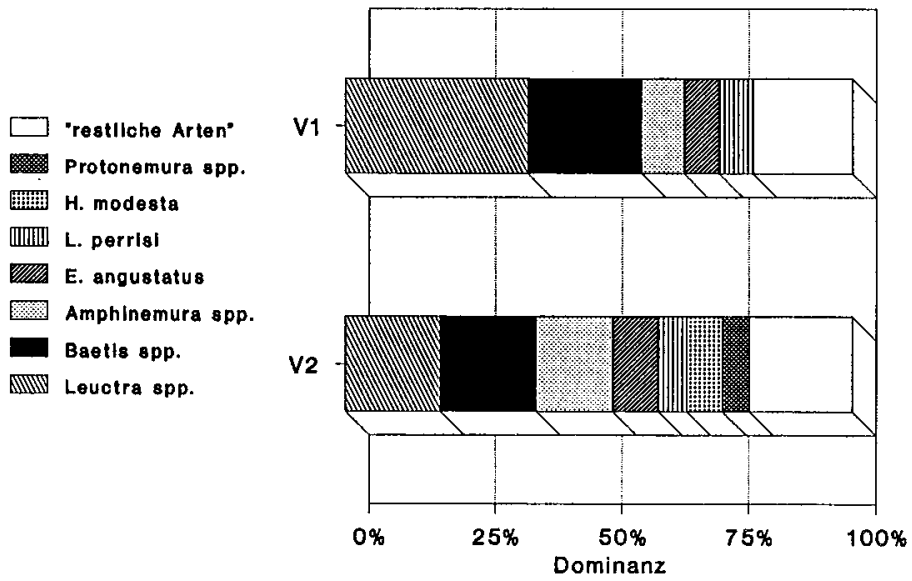

Abb. 84: Eudominante und dominante Arten an den Probestellen des Varleybachs 


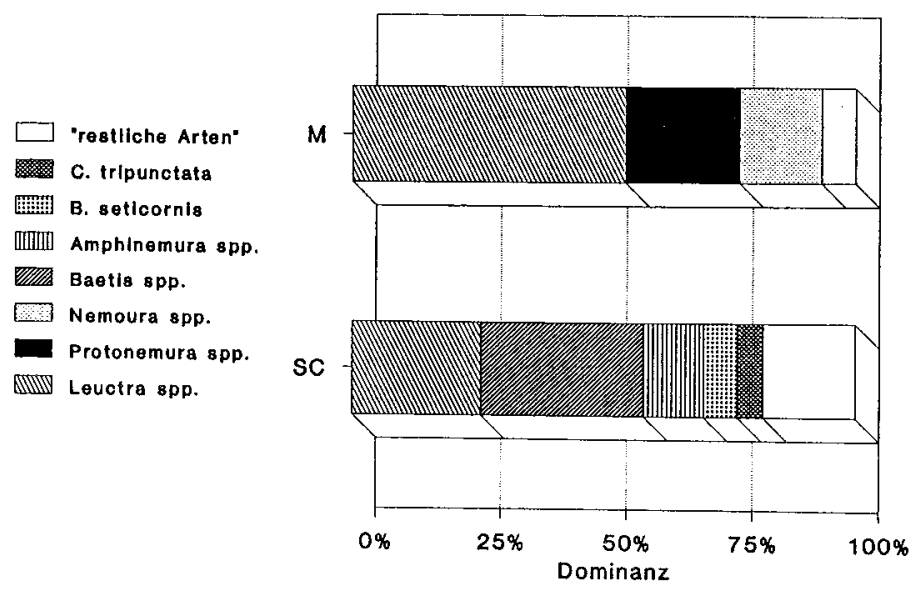

Abb. 85: Eudominante und dominante Arten an den Probestellen von Großem Mollenbach und Großer Schacht
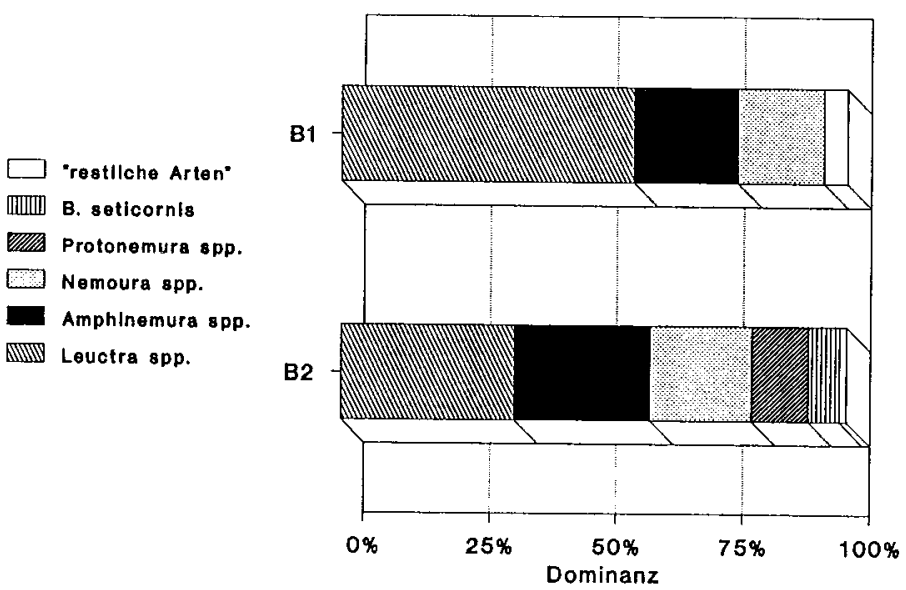

Abb. 86: Eudominante und dominante Arten an den Probestellen von Großer Bode und Warmer Bode 

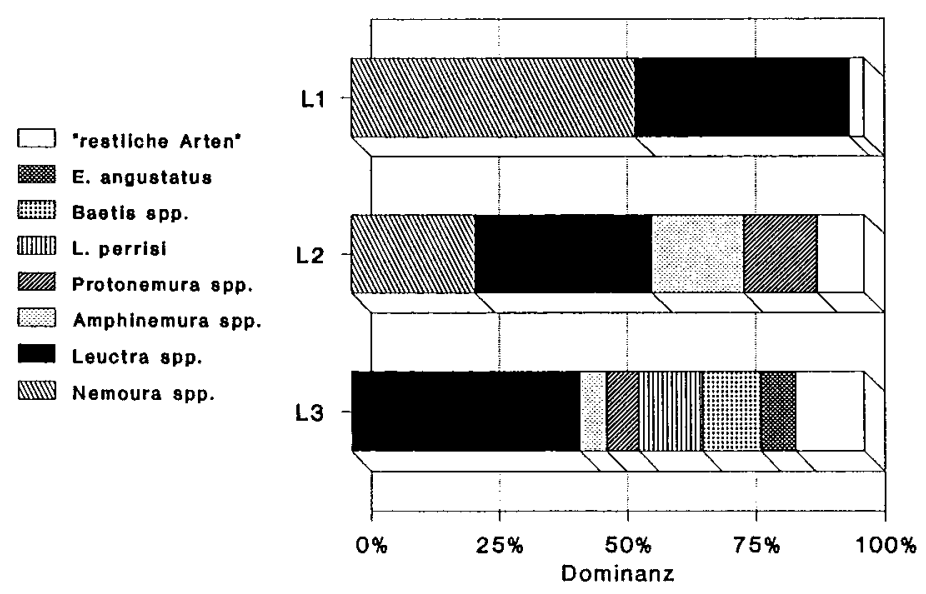

Abb. 87: Eudominante und dominante Arten an den Probestellen der Langen Bramke

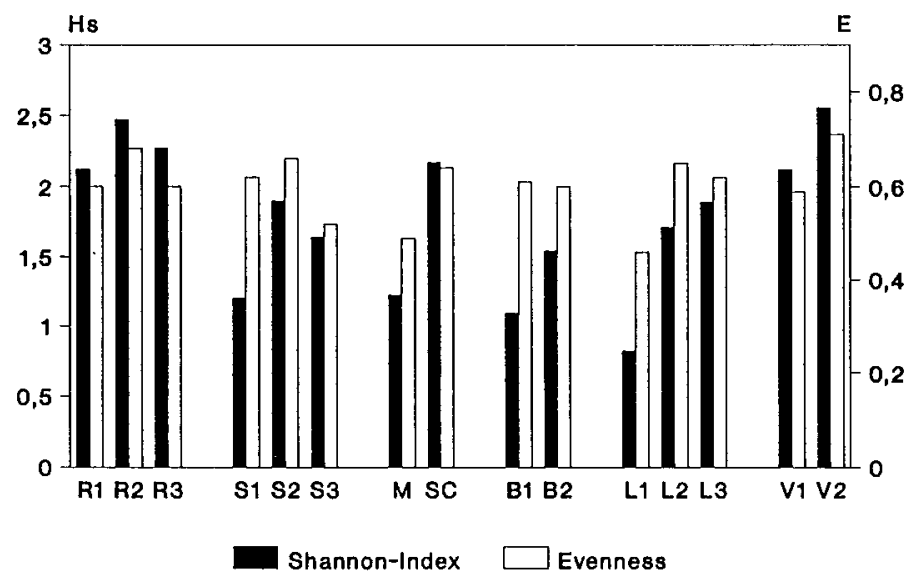

Abb. 88: Diversitäts-Indices und Evenness der Probestellen auf der Grundlage der gemeinsamen Benthosbeprobungen 


\subsection{Emergenzerfassung}

5.5.1 Emergenz der Hauptuntersuchungsbäche

\subsubsection{Artenzahlen}

In der Emergenz der Alten Riefensbeek wurden an den Probestellen je 9 Ephemeropteren- und 22 bis 25 Plecopteren-Arten gefunden. An R1 und R2 fehlen mit Baetis melanonyx, Ecdyonurus torrentis und Ephemerella mucronata sowie zusätzlich Rhithrogena semicolorata an R1 und Ephemerella ignita an R2 jeweils vier Ephemeropteren-Arten, die im Benthos nachgewiesen werden konnten. An R3 kamen Baetis scambus, Epeorus syivicola und Ephemerella mucronata im Emergenzfang nicht vor. Von den Plecopteren konnten ausschließlich Arten der großen und in relativ geringer Dichte vorkommenden Setipalpia in der Emergenz nicht gefangen werden, an R1 Dinocras cephalotes und Diura bicaudata, an R2 Dinocras cephalotes und Perlodes microcephalus sowie an R3 zusätzlich zu den Arten der oberhalb gelegenen Probestellen Isoperla.

In der Großen Söse kann sich nur eine äußerst artenarme EphemeropterenZönose entwickeln. An S2 wurden die beiden Arten Baetis vernus und Paraleptophlebia submarginata gefangen. An S3 treten mit Baetis vernus, Baetis rhodani und Baetis scambus ausschließlich Baetiden in der Emergenz auf. Im Benthos von $\$ 2$ wurden neben den aufgeführten Arten auch Siphlonurus aestivalis, Baetis rhodani und Ecdyonurus sp. sowie an $\$ 3$ Epeorus sylvicola, Rhithrogena semicolorata und Ecdyonurus venosus gefunden. Von den Plecopteren-Arten fehlen in der Emergenz aller drei Probestellen die im Benthos der gesamten Großen Söse vorkommende Isoperla sp., an S2 zusätzlich Chloroperla tripunctata, an S3 Perlodes microcephalus und Perla marginata.

\subsubsection{Emergenzdichten}

Über den gesamten Emergenz-Erfassungszeitraum betrachtet, wurden in der Alten Riefensbeek 15631 Ind./m² und in der Großen Söse 17500 Ind./m $/=$ $112 \%$ der Emergenz-Summe der Alten Riefensbeek) gefangen. In der Alten Riefensbeek tragen dabei die einzelnen Probestellen mit relativ gleichen Anteilen $(4971$ - 5501 Ind./m²) zu der Summe bei (Abb. 89). Eine starke Abnahme der Emergenzdichte ist hingegen in der Großen Söse von S1 nach S3 zu verzeichnen, wobei S1 $180 \%$ des Mittelwertes der Probestellen der Alten Riefensbeek 


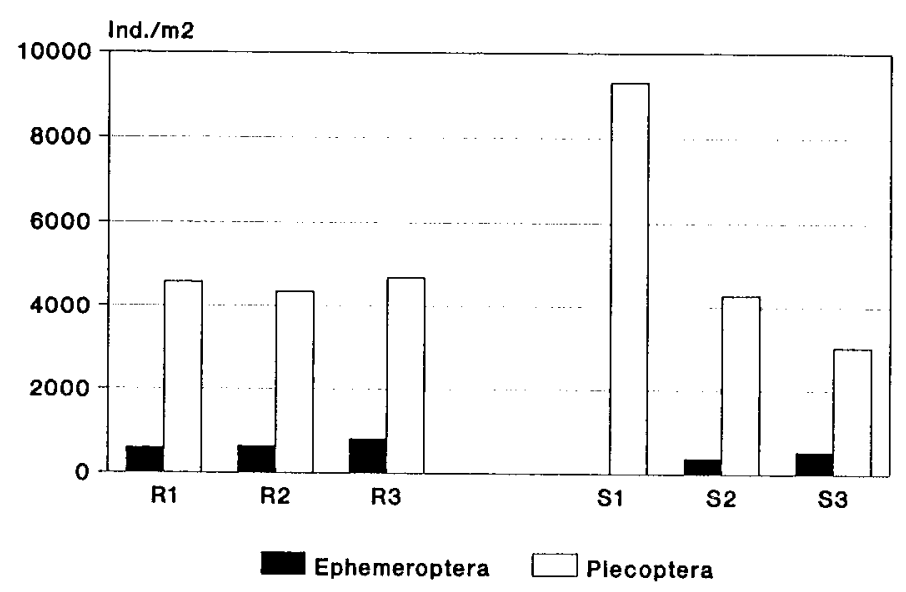

Abb. 89: Summe der Ephemeropteren- und Plecopteren-Emergenz des gesamten Erfassungszeitraums an den Hauptuntersuchungsbächen

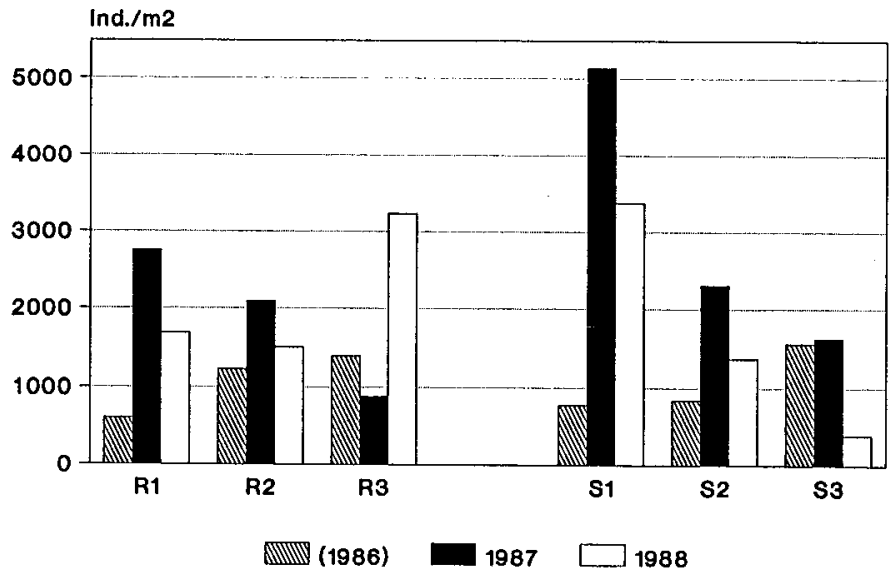

Abb. 90: Gesamtemergenzsummen der Einzeljahre 
erreicht. S2 liegt im Bereich des Wertes von R2, S3 hingegen mit nur 3555 Ind. $/ \mathrm{m}^{2}$ deutlich unter dem Wert von R3.

Die getrennte Betrachtung von Ephemeropteren und Plecopteren zeigt, daß in der Großen Söse nur $44 \%$ der Ephemeropteren-Individuenzahlen der Alten Riefensbeek gefangen wurden. Bei den Plecopteren waren es hingegen aufgrund des sehr hohen Wertes an S1 $122 \%$. Im Vergleich der einzelnen Probestellen weist die Alte Riefensbeek einen relativ konstanten Ephemeropteren-Anteil von 11,3 - 14,7 \% bei leicht ansteigender Tendenz von R1 nach R3 auf. In der Großen Söse findet bei den Ephemeropteren nach dem völligen Fehlen an $\$ 1$ von S2 nach S3 fast eine Verdoppelung des Anteils von 7,8 auf 14,5\% statt. Damit erreicht S3 die Werte der Alten Riefensbeek bei allerdings niedrigerer Besiedlungsdichte.

Im Vergleich der beiden vollständig erfaßten Jahre 1987 und 1988 ergeben sich für beide Gewässer große Schwankungen der Emergenzdichten (Abb. 90). Außer an R3 wurde 1987 an allen Probestellen eine um 64 (R1), 39 (R2), 52 (S1), 69 (S2) bzw. $318 \%$ (S3) höhere Individuenzahl gefangen als 1988. An R3 waren es 1988 bei einer starken relativen Zunahme der Zahlen sowohl der Plecopteren als auch der Ephemeropteren $267 \%$ mehr als 1987. Beide Insektenordnungen tragen, abgesehen von S1, wo nur Plecopteren vorkommen, auch zu dem Rückgang an den anderen Probestellen bei. Für die Große Söse ergibt sich, wie für den Gesamtuntersuchungszeitraum auch, in den beiden Einzeljahren eine starke Abnahme der Emergenz mit zunehmender Quellentfernung. In der Alten Riefensbeek ist dies auch $1987 \mathrm{zu}$ beobachten, 1988 wird an R3 jedoch eine um etwa 50 \% höhere Emergenzdichte erreicht als an R1 und R2, die in diesem Jahr fast gleich hohe Zahlen aufweisen.

\subsubsection{Dominanzstrukturen}

Die Analyse der Dominanzstrukturen beider Bäche über den gesamten Erfassungszeitraum betrachtet, zeigt in einigen Punkten gravierende Unterschiede zwischen dem versauerten und dem unversauerten Gewässer (Tab. 18, Abb. 91 - 96). Eudominante oder dominante Arten der Alten Riefensbeek sind Leuctra inermis und Leuctra aurita, die in der Großen Söse nur an S3 einen Anteil über 5 $\%$ an der Emergenz haben. An R1 treten daneben noch Amphinemura sulcicollis und Protonemura nitida eudominant auf. In der Großen Söse kommen beide 
Tab. 18: Dominanzstrukturen der Emergenz der Hauptuntersuchungsbäche

$\begin{array}{lll}\text { Art } & \text { Alte Riefensbeek } & \text { Grosse Söse } \\ \text { Präsenz Dominanz } & \text { Präsenz Dominanz }\end{array}$

\begin{tabular}{|c|c|c|c|c|c|c|}
\hline 1. & Leuctra inermis KFMPNY & 100 & 25,9 & 67 & 4,6 & 8. \\
\hline 2. & Leuctra aurita NAVAS & 100 & 23,2 & 67 & 2,8 & 10. \\
\hline 3. & Baetis spp. & 100 & 9,0 & 67 & 7,4 & 5. \\
\hline 4. & Amphinemura sulcicollis (SIEPHENS) & 100 & 5,7 & 67 & 0,1 & 18. \\
\hline 5. & Protonemura nitida (SIFPHENS) & 100 & 5,1 & 0 & & \\
\hline 6. & Leuctra albida KEMPNY & 100 & 4,5 & 67 & 0,1 & 19. \\
\hline 7. & Protonemura intricata (RIS) & 100 & 4,1 & 0 & & \\
\hline 8. & Protonemura auberti ILLIES & 100 & 3,4 & 100 & 10,8 & 4. \\
\hline 9. & Leuctra pseudosignifera AUBERT & 100 & 3,1 & 100 & 3,7 & 9. \\
\hline 10. & Leuctra braueri KEMPNY & 67 & 1,8 & 0 & & \\
\hline & Habrophlebia lauta EATON & 100 & 1,8 & 0 & & \\
\hline 12. & Leuctra sp. & 100 & 1,7 & 100 & 1,0 & 12. \\
\hline 13. & Leuctra nigra (OLIVIER) & 100 & 1,3 & 100 & 2,5 & 11. \\
\hline 14. & Leuctra prima KEMPNY & 100 & 1,1 & 100 & 0,5 & 16. \\
\hline & Brachyptera seticornis (KLAPAIEX) & 100 & 1,0 & 67 & 0,9 & 13. \\
\hline 16. & Protonemura nimborum (RIS) & 100 & 1,0 & 0 & & \\
\hline 17. & Siphonoperla torrentium (PICTET) & 100 & 0,9 & 67 & 5,7 & 6. \\
\hline 18. & Ecdyomurus venosus FABRICIUS & 100 & 0,6 & 0 & & \\
\hline 19. & Ephemerella ignita PODA & 100 & 0,6 & 0 & & \\
\hline & Nemurella pictetii (KLAPALEK) & 100 & 0,6 & 100 & 0,7 & 14. \\
\hline & Leuctra hippopus KEMPNY & 100 & 0,5 & 33 & 0,1 & 20. \\
\hline & Nemoura cambrica SIEPHENS & 100 & 0,5 & 100 & 4,8 & 7. \\
\hline 23. & Anphinemura standfussi (RIS) & 67 & 0,4 & 100 & 11,6 & 3. \\
\hline 24. & Leuctra pseudocingulata MENDL & 67 & 0,4 & 100 & 12,6 & 2. \\
\hline 25. & Habroleptoides modesta HACEN & 100 & 0,3 & 0 & & \\
\hline 26. & Isoperla sp. & 100 & 0,2 & 0 & & \\
\hline 27. & Protonemura hrabei RAUSER & 67 & 0,2 & 33 & 0,1 & 23. \\
\hline 28. & Rhithrogena semicolorata aRTIS & 100 & 0,2 & 0 & & \\
\hline 29. & Ohloroperla tripunctata (SOOPOLI) & 67 & 0,1 & 67 & 0,6 & 15. \\
\hline 30. & Epeorus sylvicola PICIEI & 100 & 0,1 & 33 & 0,0 & 25 . \\
\hline 31. & Leuctra fusca LTNNAEIS & 33 & 0,1 & 0 & & \\
\hline 32. & Leuctra major BRANCK & 33 & 0,1 & 33 & 0,1 & 21. \\
\hline 33. & Leuctra rauscheri AUBERT & 67 & 0,1 & 100 & 29,4 & 1. \\
\hline 34. & Nemoura cinerea (RETZIUS) & 33 & 0,1 & 0 & & \\
\hline 35. & Nemoura marginata PICIET & 66 & 0,1 & 0 & & \\
\hline 36. & Protonemura meyeri PICIKT & 67 & 0,1 & 33 & 0,1 & 22. \\
\hline 37. & Cloeon sp. & 33 & 0,0 & 0 & & \\
\hline 38. & Diura bicaudata (LNNAEUS) & 100 & 0,0 & 100 & 0,3 & 17. \\
\hline & Perla marginata (PANZER) & 33 & 0,0 & 0 & & \\
\hline & Perlodes sp. & 100 & 0,0 & 0 & & \\
\hline & Paraleptophlebia submarginata (SIEPHENS) & 0 & & 33 & 0,1 & 24. \\
\hline
\end{tabular}


Arten nur in geringer Anzahl bzw. gar nicht vor. Die verschiedenen Baetis-Arten, als wichtigste Vertreter der Ephemeropteren, erreichen zusammen an allen Stellen der Alten Riefensbeek dominante Anteile. Für die Große Söse typische Arten sind Leuctra rauscheri, Amphinemura standfussi und Leuctra pseudocingulata, die in der Alten Riefensbeek an R3 völlig fehlen und an R1 und R2 nur rezendent oder subrezedent auftreten. Die für alle drei Stellen der Großen Söse ebenfalls typische Protonemura auberti ist an R1 ebenfalls dominant. Einen von der Versauerung scheinbar unbeeinträchtigten Längsgradienten zeigt insbesondere Leuctra nigra, die in beiden Bächen in der Reihenfolge subdominant, rezedent, subrezedent vorkommt. Einen ähnlichen Gradienten weist auch Leuctra pseudosignifera in beiden Gewässern auf.

Einzelne eudominante Arten können in beiden Bächen sehr hohe Anteile erreichen: Leuctra aurita an R2 $41 \%$, Leuctra inermis an R3 $47 \%$, Leuctra rauscheri an $\mathrm{S} 160 \%$. In der Alten Riefensbeek steigt der Anteil der eudominanten Arten an der Gesamtabundanz von R1 nach R3 von 43 auf $65 \%$ an, wohingegen er in der Großen Söse an S1 und S3 bei 73 bzw. $77 \%$ liegt. An S2 werden von den eudominanten Arten zwar nur $53 \%$ erreicht, zusammen mit den dominanten sind es jedoch bereits $92 \%$ der Gesamtabundanz.

Beim Vergleich der einzelnen Jahre ergeben sich die größten Veränderungen in der Dominanzstruktur für die Probestelien S3 und R3. Dominierende Plecopteren-Arten der Probestelle R1 sind in jedem Jahr Leuctra inermis, Amphinemura sulcicollis (Hauptemergenz lag 1986 vor dem Beginn der Erfassung), Protonemura nitida, Leuctra aurita und Protonemura auberti (Abb. 91). Zu dieser relativ festgefügten Gruppe eudominanter und dominanter Arten stoßen nur vereinzelt andere sonst weniger häufige Arten. Auch an R2 wird die Dominanzstruktur in jedem Jahr weitestgehend von denselben Arten bestimmt: Leuctra aurita, Leuctra inermis und Leuctra albida (Abb. 92). Wesentlich uneinheitlicher stellen sich die Dominanzverhältnisse an R3 dar (Abb. 93). Weitaus häufigste Art ist zwar in jedem Jahr Leuctra inermis, die als Frühjahrsschlüpfer 1986 wegen der erst im Juli beginnenden Emergenzerfassung unterrepräsentiert ist, daneben treten von Jahr zu Jahr wechselnde Arten hervor, unter denen sich Leuctra aurita und Leuctra albida herausheben.

Kaum Veränderungen in der Dominanzstruktur sind an S1 zu verzeichnen, wo in jedem Jahr Leuctra rauscheri, Protonemura auberti und Amphinemura standfussi mit die höchsten Dominanzanteile besitzen (Abb. 94). Ebenfalls sehr festgefügt 
ist die Dominanzstruktur an $\mathrm{S} 2$ durch Leuctra rauscheri, Leuctra pseudocingulata, Amphinemura standfussi, Nemoura cambrica (als Frühjahrsschlüpfer 1986 unterrepräsentiert) und Protonemura auberti (Abb. 95). Wie schon R3 zeigt sich auch die unterste Probestelle der Großen Söse als die Stelle des Gewässers mit den größten jährlichen Veränderungen (Abb. 96). Allein Siphonoperla torrentium tritt von den Plecopteren in allen drei Jahren als dominante Art auf.

Von den Ephemeropteren gehört in der Alten Riefensbeek und an S3 die Gattung Baetis in jedem Jahr zu den dominanten Arten. 1986 erreichte Baetis an allen vier Stellen sowie an S2 einen außergewöhnlich hohen Dominanzanteil $>10 \%$, der auch mit Erfassung der Frühjahrsemergenz relativ hoch ausgefallen wäre.

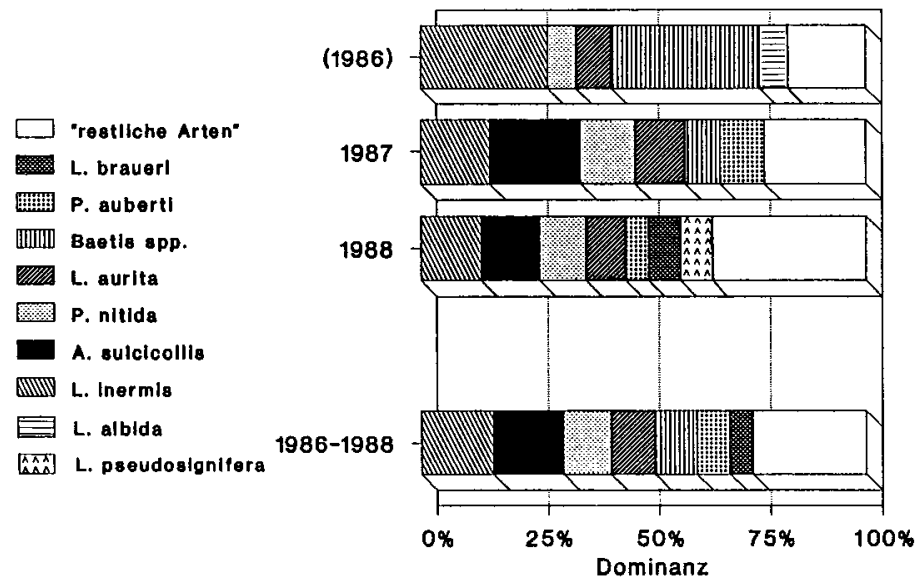

Abb. 91: Eudominante und dominante Arten der Emergenz von R1 


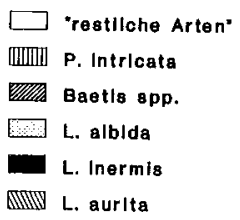

1986-1988

(1986)

1987

1988
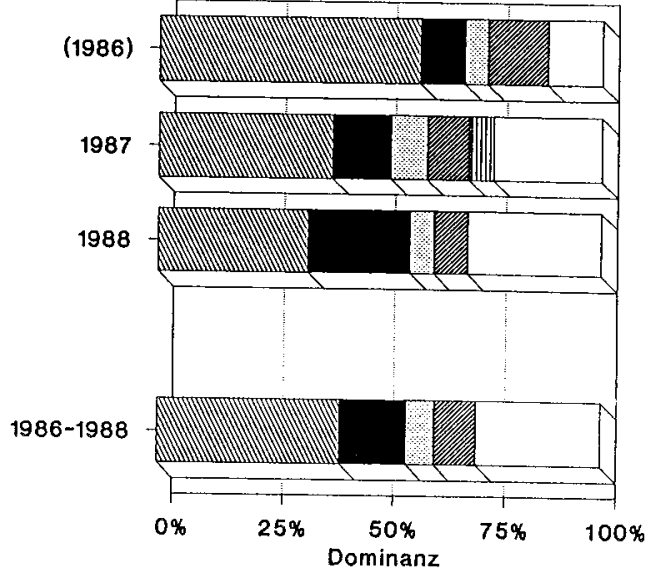

Abb. 92: Eudominante und dominante Arten der Emergenz von R2

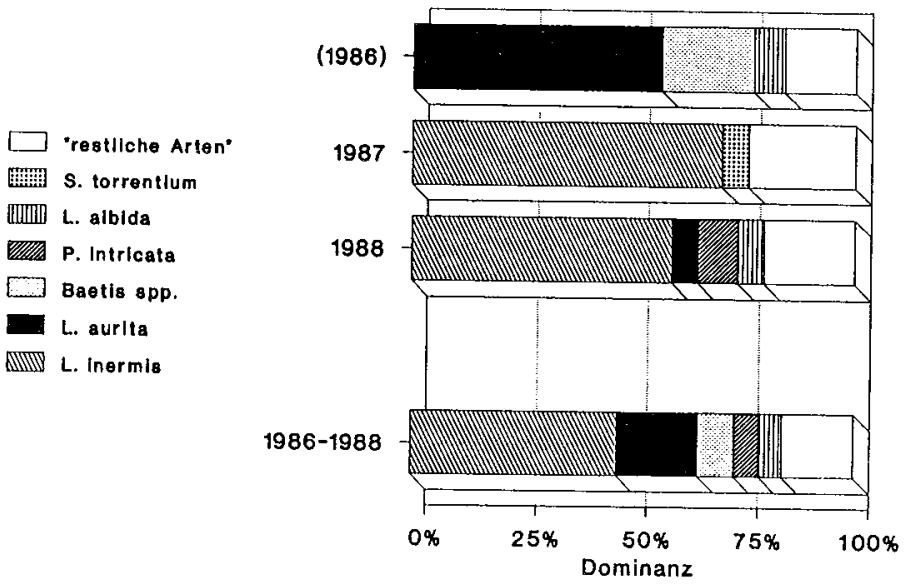

Abb. 93: Eudominante und dominante Arten der Emergenz von R3. 


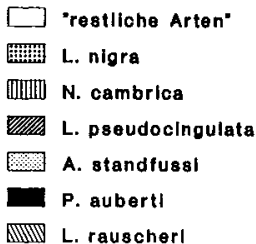

1986-1988

(1986)

1987

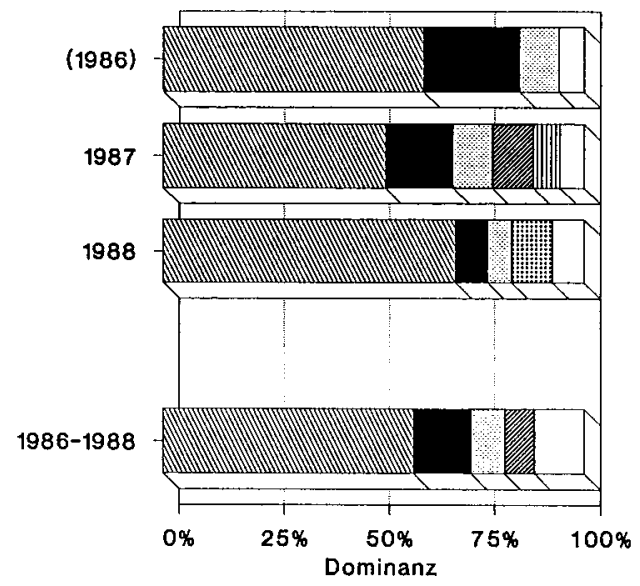

Abb. 94: Eudominante und dominante Arten der Emergenz von S1

$\square$ "restllche Arten"

Baetis spp.

典曲 P. aubertl

피미 N. cambrlca

W. pseudosignifera

A. standfuesi

L. pseudocingulata

Aivil L. rauscheri

s. torrentlum 

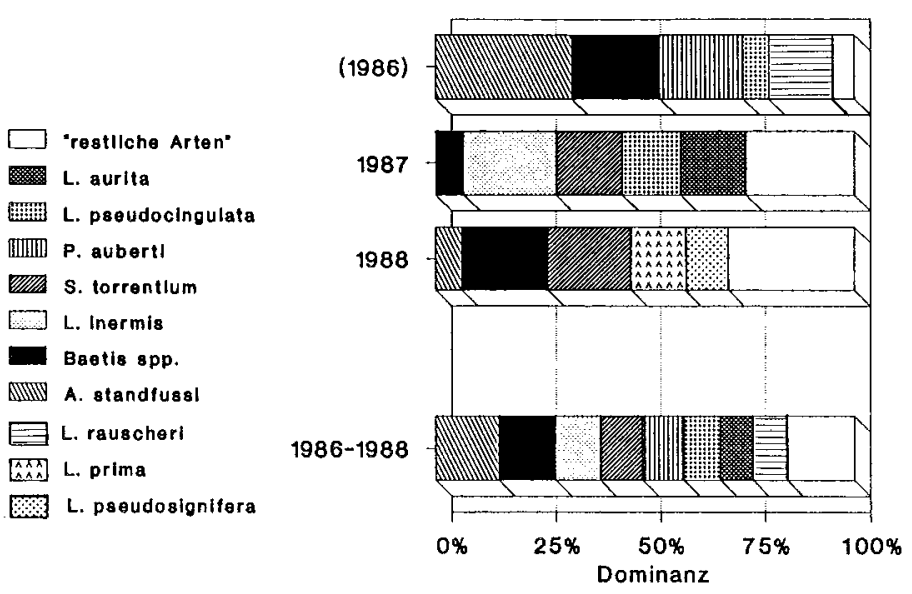

Abb. 96: Eudominante und dominante Arten der Emergenz von $\$ 3$

\subsubsection{Zeitlicher Ablauf}

Die Höhe der Emergenz wird an allen Probestellen im wesentlichen durch die stark dominierenden Arten bestimmt. Da es hohe Dominanzen einzelner Arten gibt, treten deren Emergenzmaxima an einige Stellen besonders stark hervor. Von den Plecopteren ist an den Probestellen der Alten Riefensbeek Leuctra prima im März, 1988 schon im Februar, die erste Art, die schlüpft (Abb. 97). Typische in größerer Individuenzahl vorkommende Arten der Frühjahrsemergenz sind auf den gesamten Bach bezogen in der Reihenfolge ihres Erscheinens Leuctra pseudosignifera, Leuctra inermis, Amphinemura sulcicollis und Protonemura intricata. In den Sommermonaten schließen sich Leuctra aurita, Protonemura auberti, Leuctra albida und Leuctra braueri an. Eine typische Herbstform aller drei Stellen ist Protonemura nitida.

Auch in der Großen Söse sind Leuctra prima und Leuctra pseudosignifera die ersten schlüpfenden Arten des Jahres (Abb. 98). Es folgen Leuctra nigra, Nemoura cambrica und Protonemura auberti. Zahlenmäßig bedeutende Sommer- 
arten sind Leuctra rauscheri, die vor allem für die Emergenzmaxima an $\mathbf{S 1}$ und S2 im Juni/Juli verantwortlich ist, Leuctra inermis und Siphonoperla torrentium. Ab August treten dann Leuctra pseudocingulata und Amphinemura standfussi stärker hervor und bestimmen die Emergenz bis weit in den Herbst hinein.

Wie die Plecopteren erreichen die Ephemeropteren der Alten Riefensbeek das Maximum in allen Untersuchungsjahren zwischen Juni und August, das von mehreren Baetis-Arten durch Baetis muticus, Baetis scambus und Baetis rhodani gebildet wird (Abb. 99). Wichtigste Art der Frühjahrsemergenz ist Baetis alpinus. Zu den Sommerarten trat 1988 an R3 in größerer Anzahl Habrophlebia lauta. Die Herbstemergenz wird fast ausschließlich von Baetis rhodani gestellt. In der Großen Söse wird das Emergenzgeschehen an S2 fast ausschließlich von Baetis vernus bestimmt. An $\$ 3$ treten daneben Baetis rhodani und Baetis scambus auf (Abb. 100).

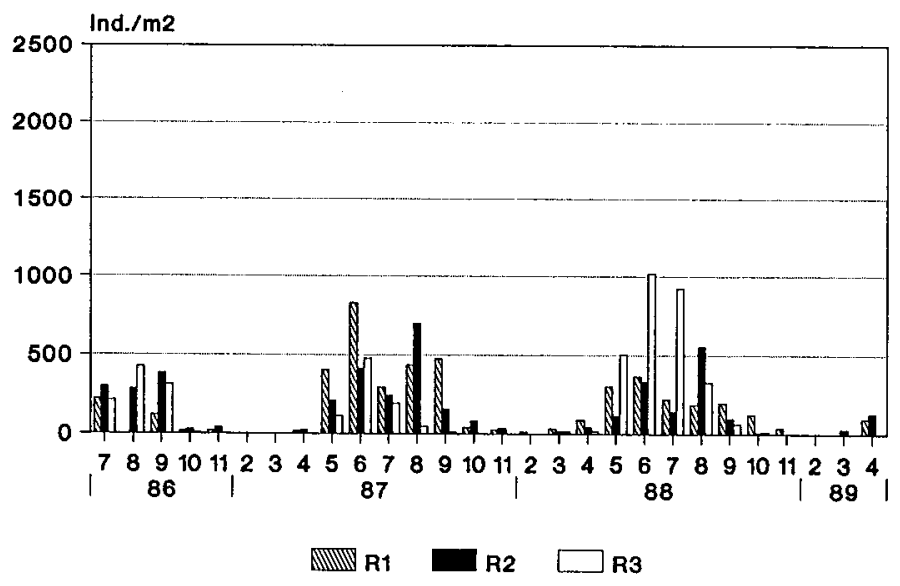

Abb. 97: Monatliche Emergenzsumme der Plecopteren an den Probestellen der Alten Riefensbeek 


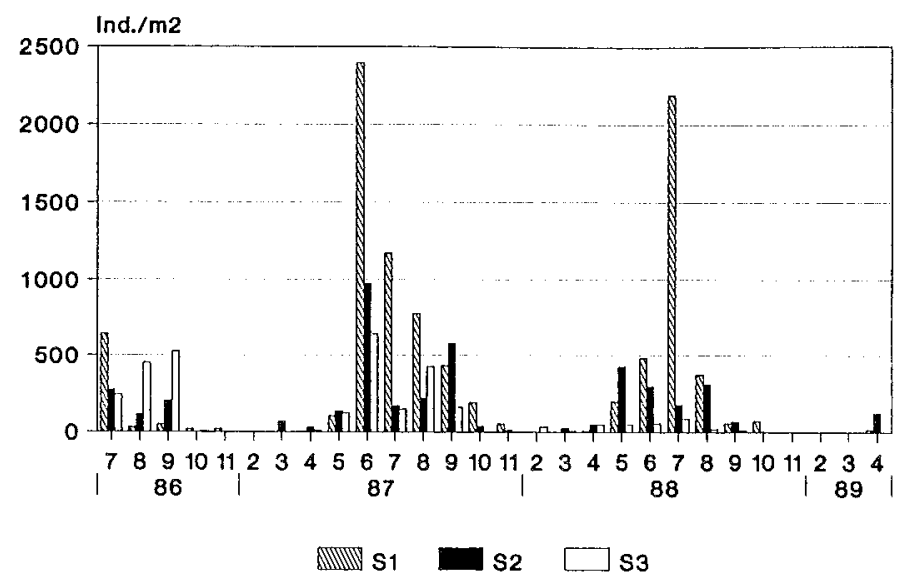

Abb. 98: Monatliche Emergenzsumme der Plecopteren an den Probestellen der Großen Söse

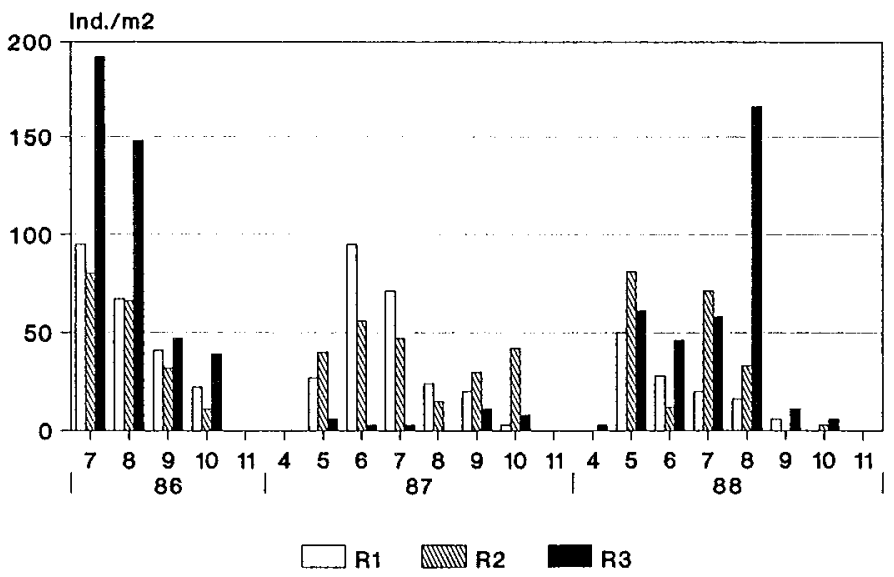

Abb. 99: Monatliche Emergenzsumme der Ephemeropteren an den Probestellen der Alten Riefensbeek 


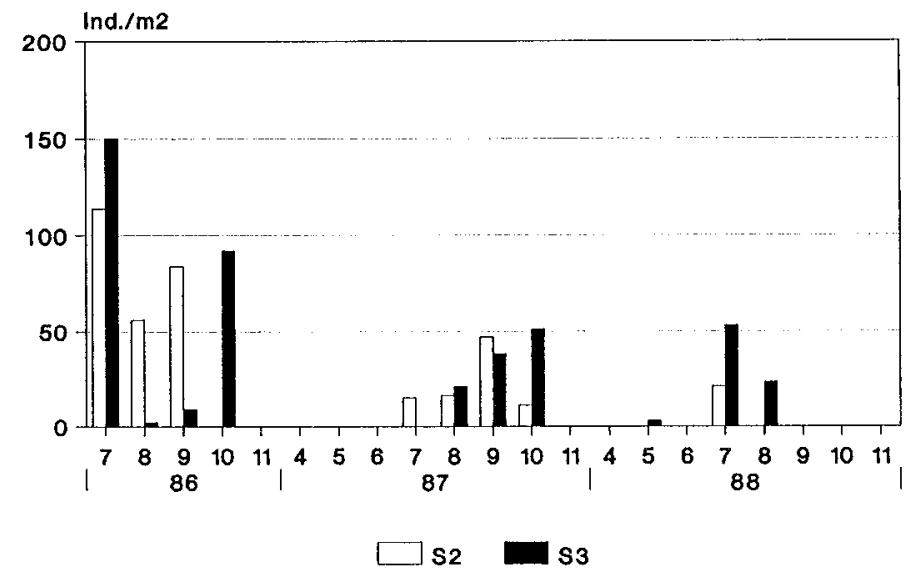

Abb. 100: Monatliche Emergenzsumme der Ephemeropteren an den Probestellen der Großen Söse

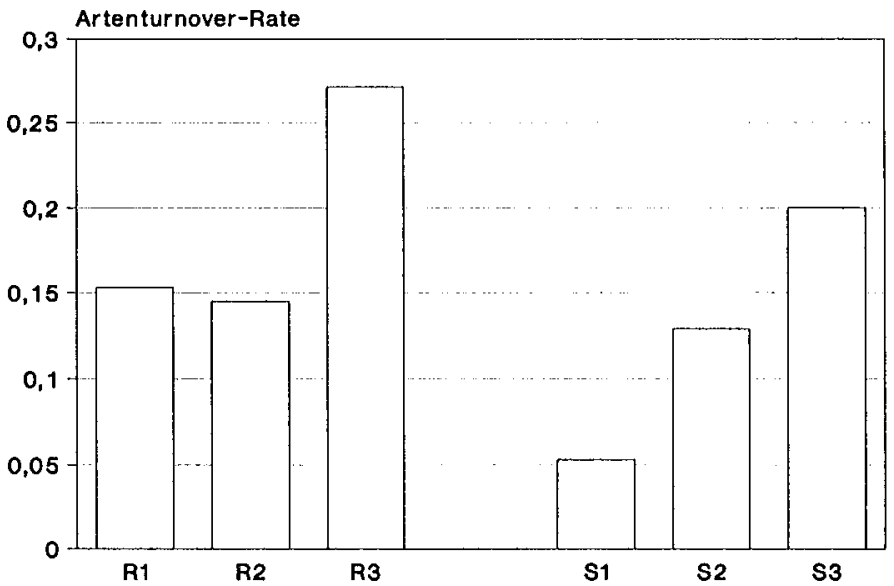

Abb. 101: Arten-Turnoverrate der Emergenz zwischen den Jahren 1987 und 1988 


\subsubsection{Biozönotischer Ähnlichkeitsvergleich}

Die dargestellten Zusammenhänge zwischen dem jährlichen Artenwechsel an den einzelnen Probestellen sowie der Ähnlichkeit in der Arten- und Dominanzstruktur der Probestellen untereinander können durch verschiedene Indices besser verdeutlicht und insbesondere quantifiziert werden. Die ArtenTurnoverrate zeigt für den Vergleich der beiden vollständig erfaßten Jahre 1987 und 1988 den weitaus stabilsten Artenbestand für $\mathrm{S} 1$ an (Abb. 101). Im Mittelfeld sind relativ dicht zusammen S2, R2 und R1 zu finden, wohingegen es bei S3 und insbesondere R3 einen großen Wechsel in der Artenzusammensetzung zu verzeichnen gab.

Der Vergleich der Probestellen hinsichtlich der Artenidentität mit Hilfe der JACCARDschen Zahl ergibt die höchsten Werte für die Probestellen der Alten Riefensbeek untereinander, wobei die jeweils am nächsten gelegenen Stellen die höchsten Werte aufweisen (Tab. 19). Den größten Unterschied in der Artenzusammensetzung zu den Probestellen der Alten Riefensbeek zeigt S1. Die Unterschiede zwischen den einzelnen Probestellen sind in der Großen Söse wesentlich größer als in der Alten Riefensbeek.

Neben der Artenidentität ist die Dominanzidentität, wie sie durch die RENKONEN-Zahl beschrieben werden kann, der zweite wichtige Parameter im Vergleich der Zoozönosestrukturen (Tab. 20). Die bereits erfolgte Darstellung der Dominanzstrukturen wird unterstrichen durch die Ergebnisse der Berechnungen. Die höchsten Dominanzidentitäten ergeben sich für die jeweils nächstgelegenen Probestellen beider Bäche, wobei sich abgesehen von S1 - S2 mit $52 \%$ für alle anderen Paare stets ein Wert von rund 57 \% errechnet. Im Vergleich der Probestellen von Großer Söse und Alter Riefensbeek ist eine sehr stark ausgeprägte Abnahme der Dominanzidentität mit zunehmender Versauerung abzulesen. Alle drei Probestellen der Großen Söse weisen den höchsten Wert zu R1 auf, so daß sich Gemeinsamkeiten einer Längszonierung nicht widerspiegeln.

Ein direkter, quantifizierender Vergleich der Biozönosen ist mit Hilfe des WAINSTEIN-Index möglich. Die Werte lassen sich anhand einer Cluster-Analyse mit Dendrogramm-Darstellung grafisch verdeutlichen (Tab. 21, Abb. 102). Versauertes und unversauertes Gewässer besitzen deutlich voneinander getrennte Zoozönosestrukturen. In der Alten Riefensbeek setzt sich R3 leicht von R1 und R2 ab, wohingegen in der Großen Söse die permanent versauerte 
Tab. 19: JACCARD-Artenidentität (untere linke Hälfte) und Anzahl der in der Emergenz gemeinsam vorkommenden Arten (obere rechte Hälfte)

\begin{tabular}{l|rrrrrr} 
R1 & & 33 & 30 & 10 & 16 & 19 \\
R2 & 0,72 & & 29 & 11 & 18 & 22 \\
R3 & 0,61 & 0,64 & & 7 & 13 & 17 \\
S1 & 0,23 & 0,29 & 0,17 & & 10 & 11 \\
S2 & 0,34 & 0,45 & 0,29 & 0,43 & & 17 \\
S3 & 0,39 & 0,54 & 0,37 & 0,41 & 0,57 & \\
\hline & $\mathrm{R} 1$ & $\mathrm{R} 2$ & $\mathrm{R} 3$ & $\mathrm{~S} 1$ & $\mathrm{~S} 2$ & $\mathrm{~S} 3$
\end{tabular}

Tab. 20: Dominanzidentität nach RENKONEN

\begin{tabular}{l|rrrrrr} 
R1 & & & & & \\
R2 & 56,8 & & & & \\
R3 & 44,2 & 56,6 & & & & \\
& & & & & & \\
S1 & 13,8 & 6,4 & 1,1 & & & \\
S2 & 24,3 & 19,2 & 12,7 & 52,1 & \\
S3 & 39,5 & 34,1 & 30,7 & 37,2 & 56,8 & \\
\hline & $R 1$ & $R 2$ & $R 3$ & $S 1$ & $S 2$ & S3
\end{tabular}

Tab. 21: WAINSTEIN-Indices

\begin{tabular}{l|rrrrrr}
$\mathrm{R} 1$ & & & & & \\
$\mathrm{R} 2$ & 40,9 & & & & \\
$\mathrm{R} 3$ & 27,0 & 36,2 & & & & \\
& & & & & & \\
$\mathrm{~S} 1$ & 3,2 & 1,9 & 0,2 & & & \\
$\mathrm{~S} 2$ & 8,3 & 8,6 & 3,7 & 22,4 & $\mathrm{~S} 2,4$ & $\mathrm{~S} 3$ \\
$\mathrm{~S} 3$ & 15,4 & 18,4 & 11,4 & 15,3 & 32
\end{tabular}


Probestelle S1 nur eine geringe Ähnlichkeit zu S2 und S3 aufweist. Aus den errechneten Werten ergibt sich außerdem, daß im Vergleich der beiden Bäche der Versauerungsgrad das Maß der Ähnlichkeit zu den Stellen der Alten Riefensbeek bestimmt. Die Unähnlichkeit ist am größten zwischen allen Stellen der Großen Söse und R3. Entsprechend einer zu erwartenden Längszonierung hat S2 die größte Übereinstimmung, auf allerdings sehr niedrigem Niveau, mit R2 und S1 mit R1.

Neben diesen vergleichenden Betrachtungen sind abgesehen von der Artenzahl auch SHANNON-Index und Evenness geeignet, Aussagen über die "Qualität" der untersuchten Zoozönosen zu machen (Abb. 103). Beide Indices zeigen in den untersuchten Gewässern einen entgegengesetzten Verlauf. In der Alten Riefensbeek sinken Diversität und Evenness von R1 nach R3. In der Großen Söse steigen die Werte mit abnehmender Wasserstoffionenkonzentration an. S1 hat aufgrund der niedrigen Artenzahi mit der zusätzlichen hohen Dominanz weniger Arten den weitaus niedrigsten SHANNON-Index. Hohe Artenzahl und relativ ausgeglichene Dominanzstruktur lassen R1 den höchsten Wert erreichen, wohingegen R2 und R3 zwar auch hohe Artenzahlen aufweisen, die Dominanz einiger Arten aber sehr hoch ist und dadurch der Diversitätsindex auf Werte absinkt, die auch S2 und S3 haben bzw. sogar überschreiten.

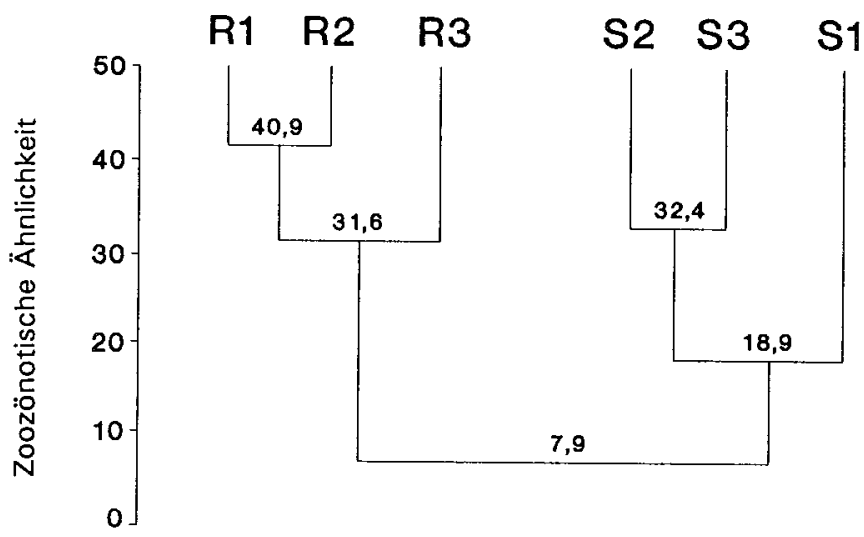

Abb. 102: Dendrogramm zur Cluster-Analyse der WAINSTEIN-Indices 


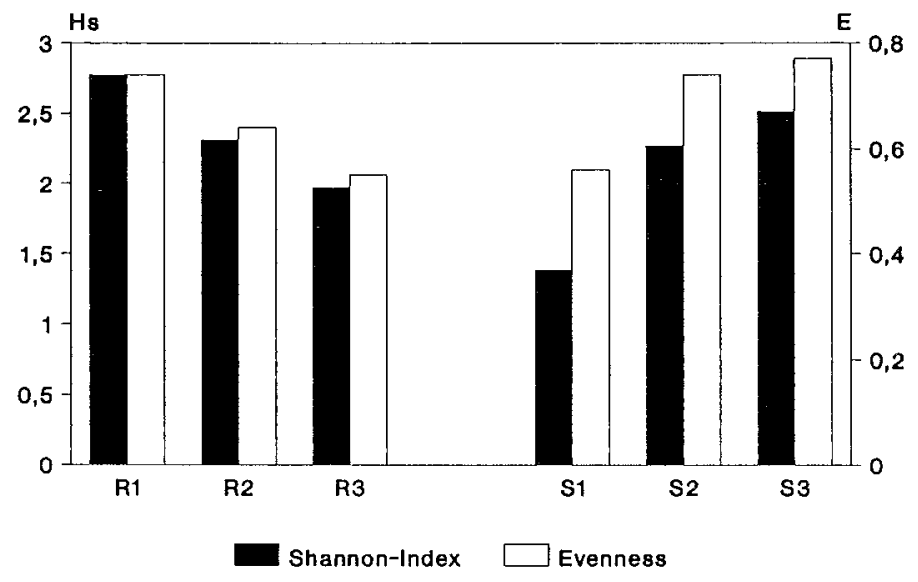

Abb. 103: Diversitäts-Indices $\left(H_{s}\right)$ und Evenness $(E)$ der Emergenz

An den beiden unteren Stellen der Großen Söse ist zwar die Artenzahl niedrig, aber die Dominanzstrukturen sind relativ ausgeglichen. Dies spiegelt sich noch stärker in der Evenness wider, die für S2 und S3 zusammen mit R1 eine hohe Gleichverteilung der vorkommenden Arten anzeigt. Die unausgeglichene Dominanzstruktur an S1 und R3 wird durch die Evenness bestätigt. Gleichzeitig muß jedoch beachtet werden, daß in der Alten Riefensbeek 31 - 34 Arten und in der Großen Söse von S1 nach S3 dagegen nur 11, 19 und 23 Arten in der Emergenz nachgewiesen werden konnten.

\subsubsection{Emergenz der Nebenuntersuchungsbäche}

Für die Zeit vom 15.06.1986 bis 31.12.1987 liegt eine gemeinsame Emergenzerfassung an allen 15 Probestellen vor. Erkenntnisse der intensiveren Untersuchungen an den Probestellen von Alter Riefensbeek und Großer Söse können dadurch auf allgemeine sich aufgrund der Gewässerversauerung abzeichnende Tendenzen überprüft werden. 


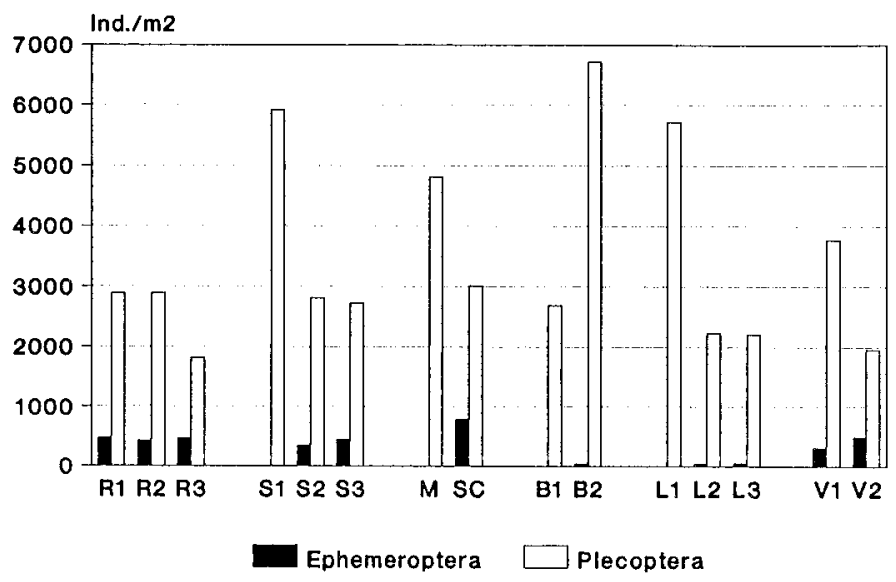

Abb. 104: Summe der Emergenz des gemeinsamen Erfassungszeitraums $1986 / 1987$

Die höchsten Artenzahlen konnten in der Emergenz der nur schwach versauerten bzw. nicht versauerten Probestellen SC, V1 und V2 mit 28, 26 und 23 Arten gefunden werden, worunter $8 \mathrm{bzw}$. am Varleybach 7 EphemeropterenArten waren. Zur Emergenz von L3 mit 22 Arten und B2 mit 20 Arten zählen dagegen bei etwa gleicher Zahl an Plecopteren-Arten nur maximal zwei Baetiden. Deutlich niedriger sind die Gesamtartenzahlen mit 11 bis 7 Arten an M, B1, L2 und L1. Ephemeropteren wurden unter diesen Stellen nur an L2 im September 1986 in Form weniger Baetis-Subimagines gefangen.

Die Gesamtemergenz nimmt, wie bereits in Großer Söse und Alter Riefensbeek gesehen, mit Ausnahme von Großer Bode/Warmer Bode mit zunehmender Quellentfernung ab (Abb. 104). Von B1 nach B2 ist hingegen ein Anstieg um das 2,5-fache auf den Spitzenwert aller Probestellen von $6756 \mathrm{lnd} . / \mathrm{m}^{2}$ festzustellen. Der niedrige Wert an B1 könnte in den für die faunistische Besiedlung ungünstigen Substratverhältnissen begründet sein. Alle anderen versauerten quellnahen Probestellen weisen deutlich höhere Emergenzdichten auf als die entsprechenden Stellen der beiden neutralen Bäche; in den mittleren und unteren Bereichen liegen die Probestellen in einer gemeinsamen Schwankungsbreite. Einen größeren Anteil an der Emergenz erreichen die Ephemeropteren mit 20,7 \% nur 
an SC und mit 19,8\% an V2. Diese Anteile liegen über den maximal $14,7 \%$ der Alten Riefensbeek. An V1 machen Ephemeropteren nur 7,4\% und an den restlichen Stellen weniger als $2 \%$ der Emergenz aus.

Trichopteren haben an allen Probestellen nur eine geringe Emergenzdichte (RÜDDENKLAU 1989). An Probestellen mit Ephemeropteren-Vorkommen liegt ihre Zahl fast immer deutlich unter der der Ephemeropteren. In den nicht versauerten Bächen wurde eine bachabwärts abnehmende Emergenz zwischen 90 und 236 Ind. $/ \mathrm{m}^{2}$ ermittelt. In allen versauerten Gewässern wurden um oder sogar weit unter $100 \mathrm{lnd} . / \mathrm{m}^{2}$ gefangen.

Für die Betrachtung der Dominanzstruktur ist insbesondere das vollständig erfaßte Jahr 1987 von Interesse. Auch in den Nebenuntersuchungsbächen erreichen unabhängig vom Grad der Gewässerversauerung einzelne Arten sehr hohe Anteile an der Emergenz. Gemeinsame eudominante Art der VarleybachProbestellen ist Leuctra aurita. An beiden Stellen ist Amphinemura sulcicollis unter den dominanten Arten (Abb. 105, 106). V1 weist daneben Leuctra inermis als eudominante Art auf. An V2 ist 1987 neben Leuctra aurita allein hier mit Habrophlebia lauta eine Ephemeroptere eudominant.

Nur in der Großen Schacht hatten die Ephemeropteren mit der Gattung Baetis 1986 ebenfalls eine sehr hohe Emergenzdichte, die 1987 jedoch nicht wieder auftrat (Abb. 108). Die großen Unterschiede in der Dominanzstruktur zu den stark versauerten Probestellen werden beim Vergleich zwischen SC und der oberhalb gelegenen Stelle $M$ deutlich, die keine gemeinsamen dominanten Arten aufweisen (Abb. 107). Eine charakteristische Art in der Dominanzstruktur ist an allen Stellen der Langen Bramke Leuctra nigra (Abb. 111 - 113). Die zweite wichtige Art dieses Baches ist Nemurella pictetii, deren Emergenzdichte bachabwärts jedoch stark zurückgeht. Gemeinsame dominante Arten von B1 und B2 sind Leuctra rauscheri, Amphinemura standfussi und Nemoura cambrica, wobei an B1 die Dominanzstruktur sehr einseitig von Leuctra rauscheri beherrscht wird (Abb. $109-110$ ). 


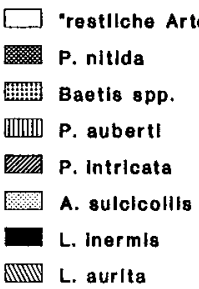

Abb. 105: Eudominante und dominante Arten des Benthos im Varleybach an V1

[- "restlliche Arten"

眖乘 Baetis spp.

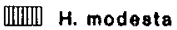

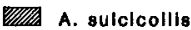

H. lauta

L. aurita

L. alblda
(1986)

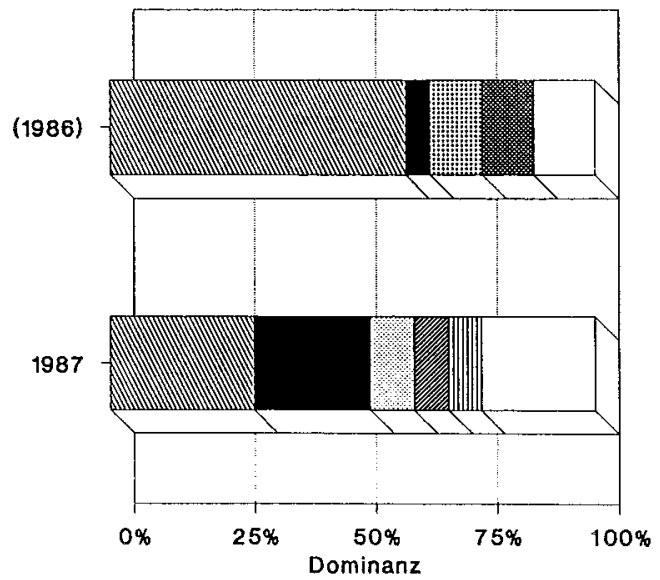

1987
(1986)

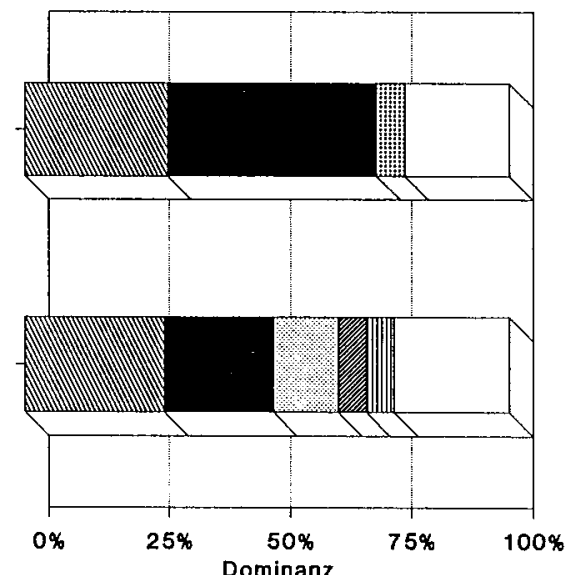

Abb. 106: Eudominante und dominante Arten des Benthos im Varleybach an V2 


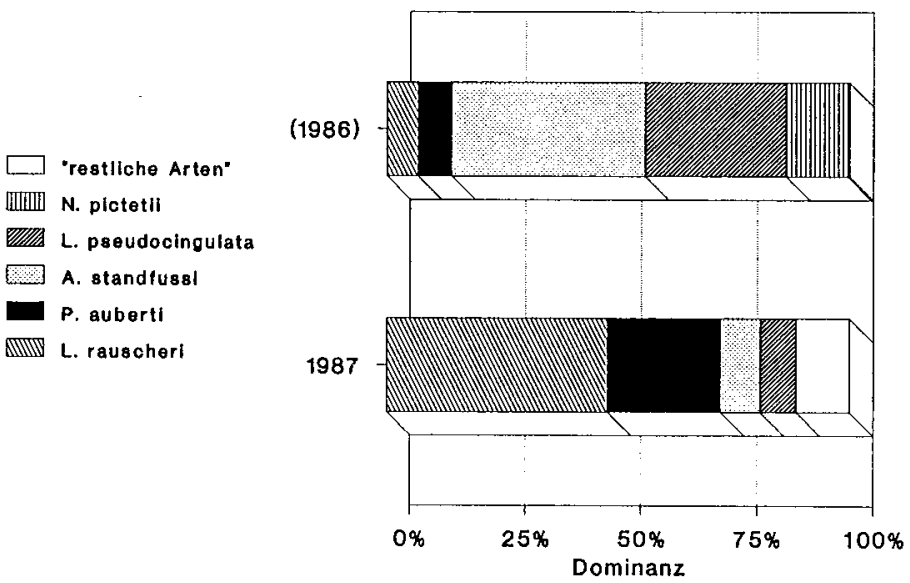

Abb. 107: Eudominante und dominante Arten des Benthos im Großen Mollenbach an $\mathrm{M}$

\section{$\square$ "restliche Arten"}

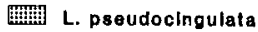
IIIIII) P. aubert!

Waetls spp.

L. inermls

C. tripunctata

Mili L. aurlta

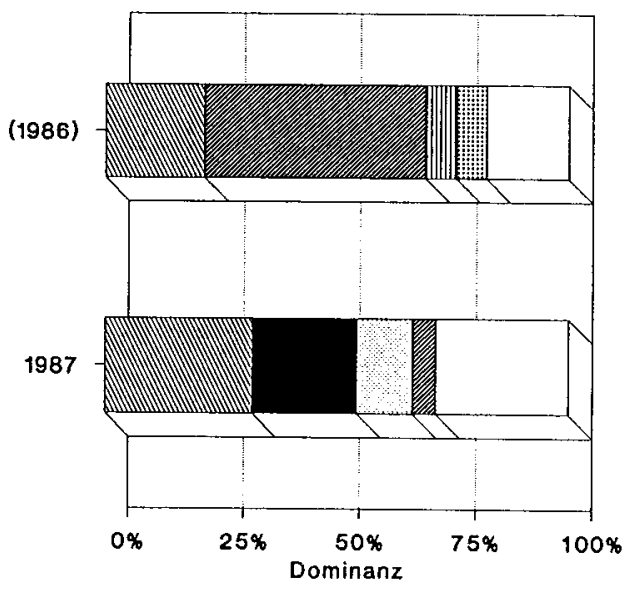

Abb. 108: Eudominante und dominante Arten des Benthos in der Großen Schacht an SC 


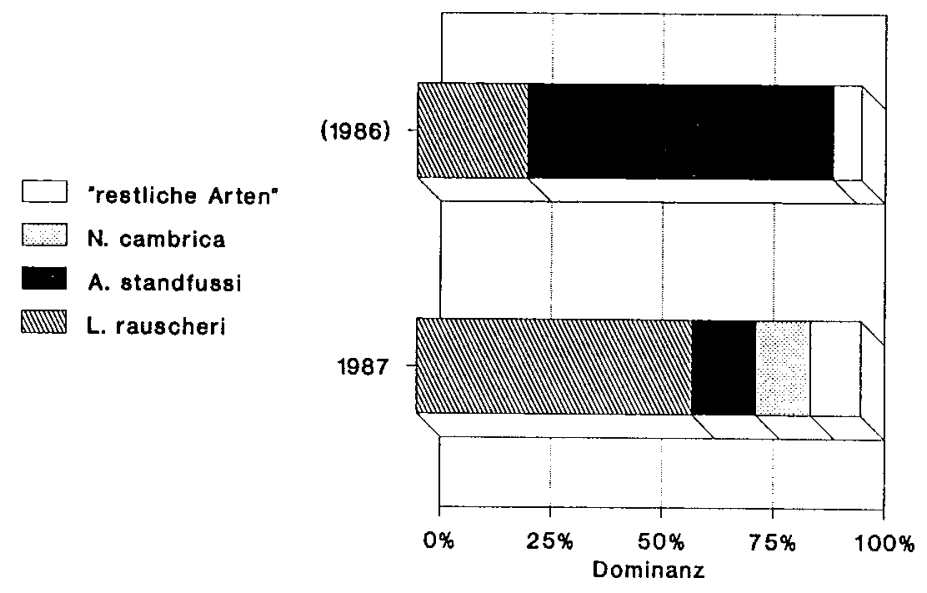

Abb. 109: Eudominante und dominante Arten des Benthos in der Großen Bode an B1
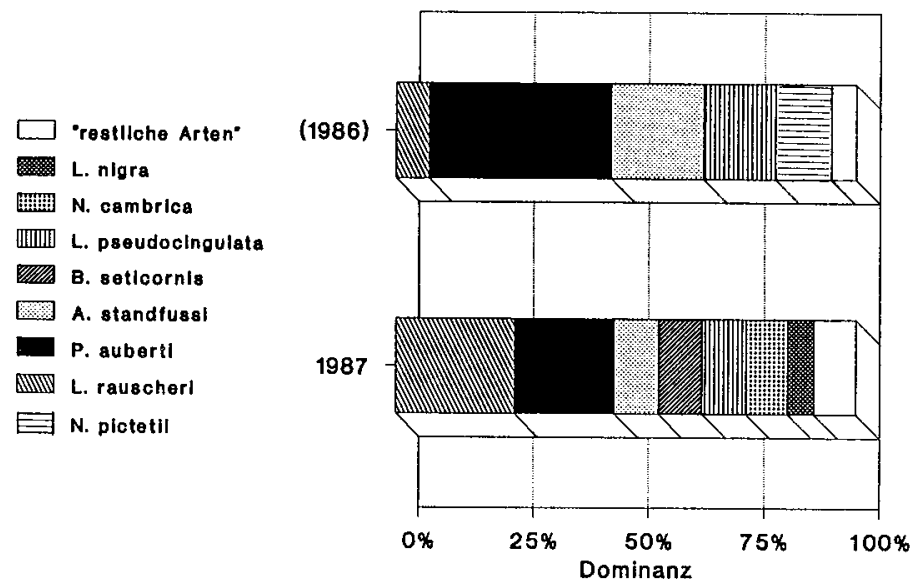

Abb. 110: Eudominante und dominante Arten des Benthos in der Warmen Bode an $\mathrm{B2}$ 


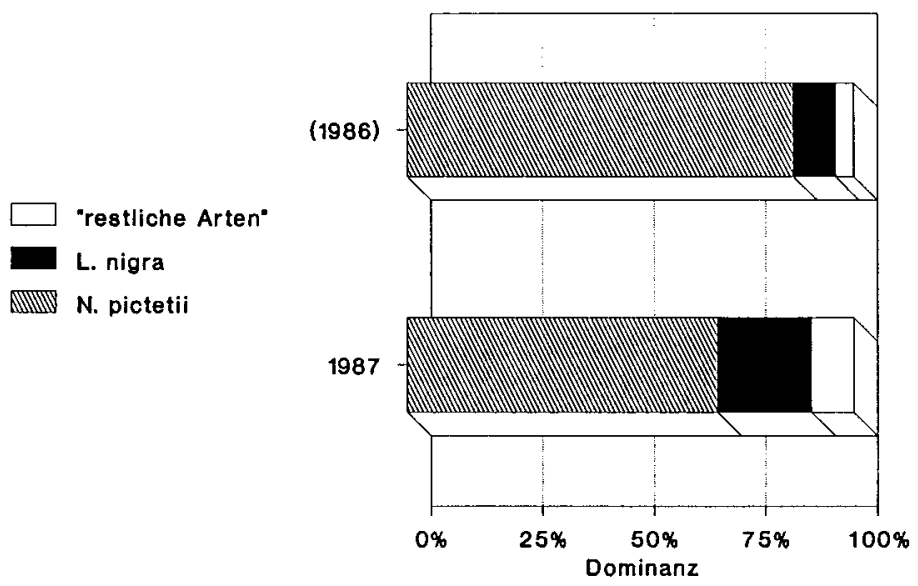

Abb. 111: Eudominante und dominante Arten des Benthos in der Langen Bramke an L1

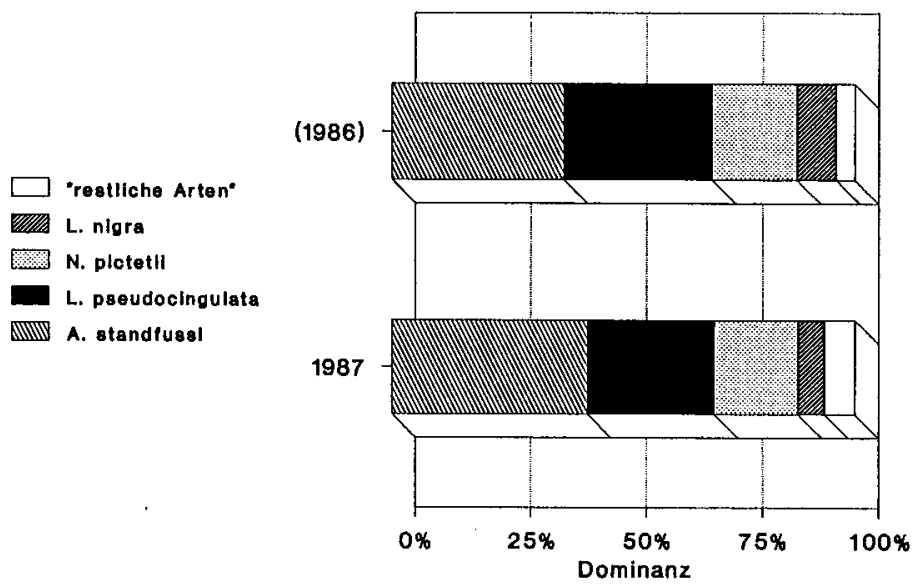

Abb. 112: Eudominante und dominante Arten des Benthos in der Langen Bramke an L2 

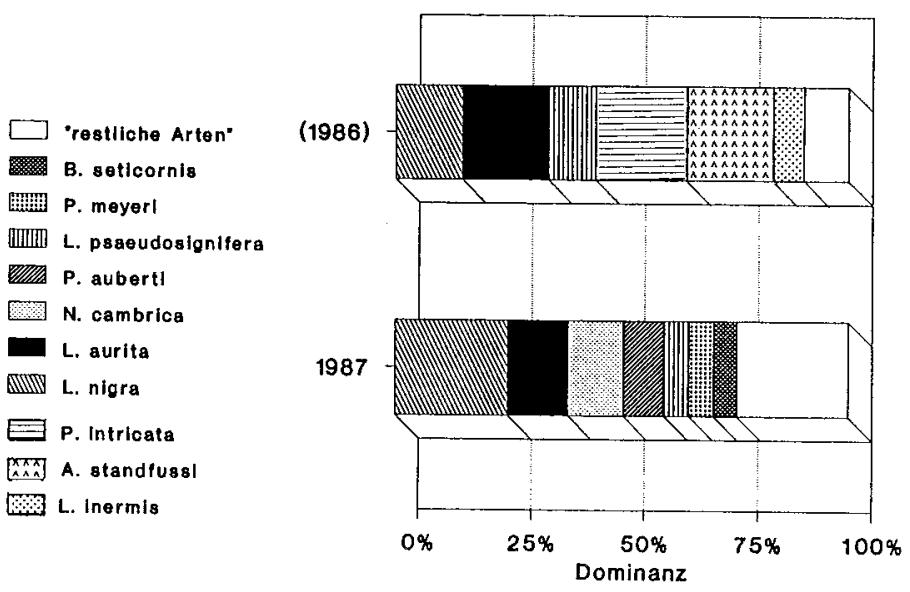

Abb. 113: Eudominante und dominante Arten des Benthos in der Langen Bramke an L3

Die Jahresperiodik der Ephemeropteren-Emergenz wird nur im Varleybach nicht allein von Baetis-Arten bestimmt. Der Kurvenverlauf spiegelt hier weitestgehend die Emergenz von Habroleptoides modesta im Frühjahr und Habrophlebia lauta im Sommer wider (Abb. 114). An den restlichen Stellen sind Baetis muticus und Baetis rhodani (SC und $V 1$ ) bzw. Baetis rhodani und Baetis vernus die bestimmenden Arten. An der schwach versauerten Probestelle SC ist das Emergenzmaximum deutlich zum Herbst hin verschoben, und an den restlichen versauerten Gewässern mit Ephemeropteren-Emergenz treten Ephemeropteren ausschließlich im August/September auf.

Das Maximum der Plecopteren-Emergenz liegt an den meisten Probestellen in den Sommermonaten (Abb. 115, 116). Die hohen Emergenzdichten im Juni sind an $M$ und B2 auf Leuctra rauscheri, an V1 und SC auf Leuctra inermis bzw. Chloroperla tripunctata und Leuctra inermis zurückzuführen. An der höher gelegenen Probestelle B1 tritt das Maximum durch Leuctra rauscheri erst im Juli auf. Die Frühjahrsemergenz an $L 1$ und $L 3$ wird von Leuctra nigra gebildet, wohingegen die Sommeremergenz an L1 vorwiegend aus Nemurella pictetii besteht. 


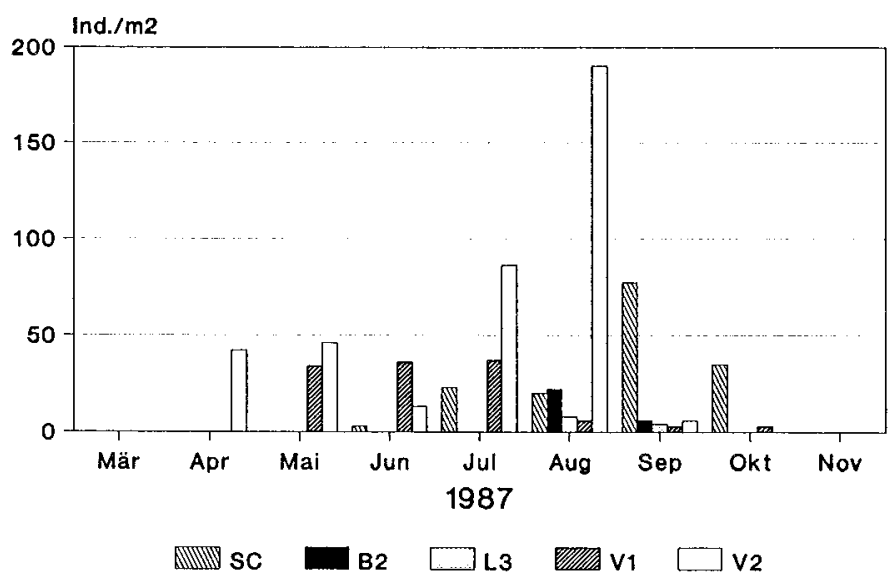

Abb. 114: Summe der monatlichen Ephemeropteren-Emergenzen

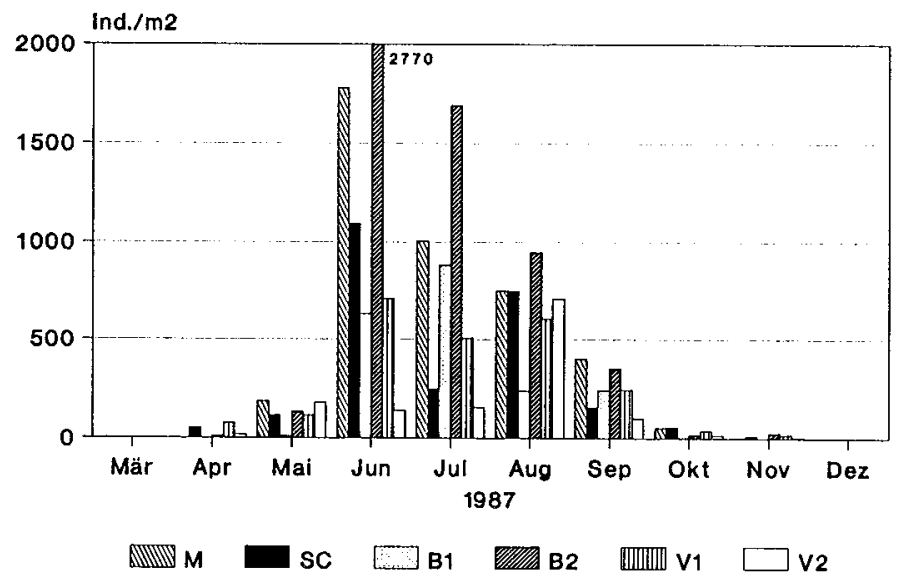

Abb. 115: Summe der monatlichen Plecopteren-Emergenzen 


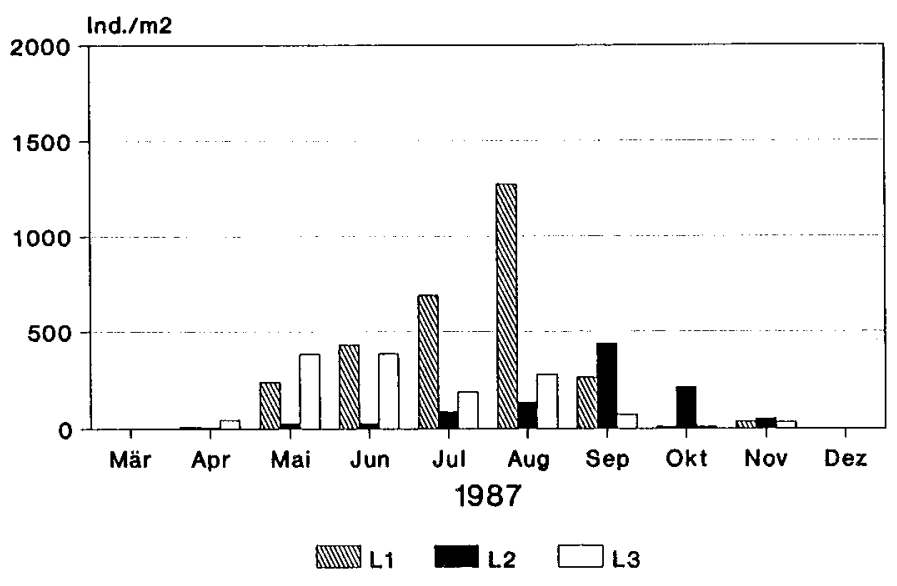

Abb. 116: Summe der monatlichen Plecopteren-Emergenzen der Langen Bramke

\subsubsection{Geschlechterverhältnis}

Beim Geschlechterverhältnis der in der Emergenz erfaßten Tiere zeigt sich keine Bezjehung zum Versauerungsgrad der Probestellen. An allen Stellen ist sowohl bei den weitaus meisten Ephemeropteren- als auch Plecopteren-Arten ein erheblicher Überschuß an Männchen festzustellen. So treten an allen Probestellen der Großen Söse und der Alten Riefensbeek die eudominanten Arten von wenigen Ausnahmen abgesehen mit einem Verhältnis von Männchen zu Weibchen auf, das weit größer ist als $10: 1$. Auch bei den dominanten und subdominanten Arten ist meist ein erheblicher Überschuß an Männchen vorhanden, der jedoch nur selten ein so krasses Ungleichgewicht zeigt wie bei den eudominanten Arten. Ein Verhältnis von $1: 1$ oder sogar leicht darunter haben fast ausschließlich subrezedente Arten, von denen einige aber auch einen Überschuß an Männchen aufweisen. Zwischen dem Geschlechterverhältnis und den Dominanzen der jeweiligen Arten im darauffolgenden Jahr sind keine direkten Beziehungen aus den vorliegenden Daten abzulesen. 
Die Ursachen für diesen hohen Überschuß an Männchen sind nicht ersichtlich. Ein methodischer Fehler bei der Erfassung kann weitgehend ausgeschlossen werden, da ein so großes Ungleichgewicht bei Verwendung der gleichen Fallen bei anderen Untersuchungen nicht beobachtet werden konnte (BLANKE 1988, RAMACHERS \& BLANKE 1987, WESTERMANN 1990). Zudem erbrachte auch die Bearbeitung der Trichopteren sowohl über die Emergenzerfassung als auch durch Licht- und Handnetzfang bei sehr vielen Arten einen hohen Überschuß an Männchen (RÜDDENKLAU 1989).

\subsubsection{Biozönotischer Ähnlichkeitsvergleich}

Eine Gesamtbetrachtung von Diversität und Evenness anhand der Emergenzdaten des gemeinsamen Erfassungszeitraums aller Probestellen zeigt, daß der SHANNON-Index mit zunehmender Versauerung eine Tendenz zur Abnahme zeigt, die allerdings nur bei den am stärksten versauerten Probestellen (S1, $M$, B1, L1) auch deutlich in Erscheinung tritt, wohingegen die Schwankungsbreite in der Gruppe der mäßig und schwach versauerten sowie nicht versauerten Stellen so groß ist, daß hier anhand des Wertes einer einzeinen Stelle kein Diversitätsverlust aufgrund der Versauerung ermittelt werden kann (Abb. 117). Wie schon an den Hauptuntersuchungsbächen festgestellt, spiegelt sich die Versauerung in der Evenness nicht wider. Der sehr niedrige Wert für L1 weist aber zusammen mit der niedrigen Diversität auf die an dieser Probestelle herrschenden, vor allem gewässermorphologisch bedingten, extremen Lebensbedingungen und die daraus resultierende nur sehr schwach differenzierte Biozönose hin.

Die Einbeziehung der Nebenuntersuchungsbäche in den biozönotischen Ähnlichkeitsvergleich auf der Grundlage des gemeinsamen Emergenzerfassungszeitraums, wie er bereits für die Hauptuntersuchungsbäche durchgeführt wurde, ermöglicht es darzustellen, inwieweit eine Differenzierung der Biozönosen aufgrund der Gewässerversauerung erfolgt.

Die JACCARD-Artenidentität ist bei sehr hohen Werten zwischen den am stärksten versauerten Probestellen (S1, B1, M) am größten, was darauf hinweist, daß sich die Biozönosen stark versauerter Probestellen durch eine relativ charakteristische Artenzusammensetzung auszeichnen (Tab. 22). Die nicht versauerten Stellen haben zwar auch relativ hohe Werte, zeigen aber 


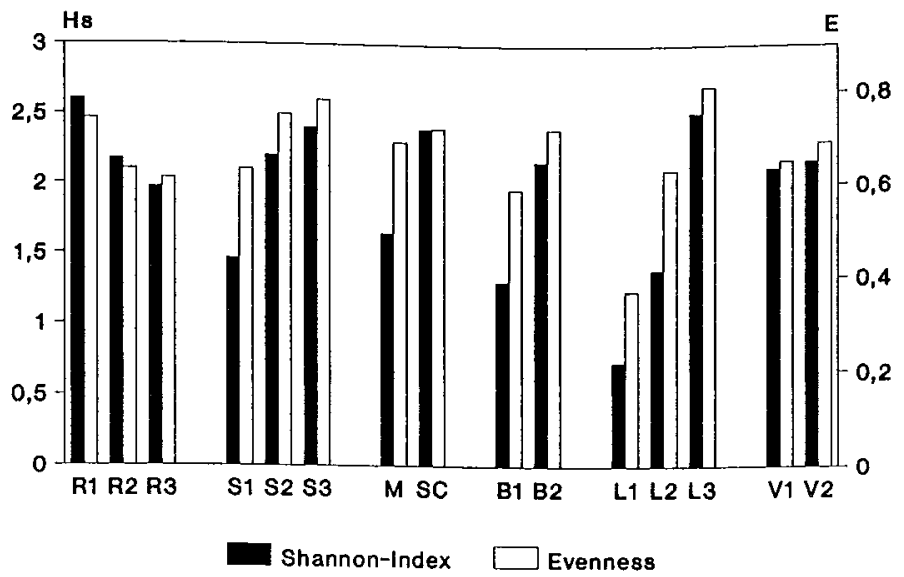

Abb. 117: Diversitäts-Indices und Evenness der gemeinsamen Emergenzerfassung

gegenüber den stark versauerten Stellen bereits größere Schwankungen im Artenbestand. Eine ebenfalls noch relativ hohe Artenidentität ergibt sich zwischen den mäßig sauren Probestellen. Die größten Unterschiede innerhalb eines Bachsystems bestehen zwischen $M$ und SC sowie zwischen den relativ dicht zusammenliegenden Stellen der Langen Bramke.

Die höchste Dominanzidentität besteht nach der RENKONEN-Zahl zwischen den stark sauren Probestellen M und S1 (Tab. 23). Allgemein errechnen sich relativ hohe Werte zwischen allen stark und mäßig sauren Stellen. Zwischen den nicht versauerten Stellen können sich dagegen größere Unterschiede ergeben (z.B. R1 - V2). Die schwach sauren Probestellen zeigen untereinander nur eine geringe Dominanzidentität. Die niedrigsten Werte sind wie bei der Artenidentität zwischen den stark sauren und den nicht versauerten Stellen zu verzeichnen. An der Langen Bramke wird die Sonderstellung von L1 deutlich, die abgesehen vom eigenen Bachsystem mit allen anderen Stellen die wenigsten Gemeinsamkeiten in der Dominanzstruktur aufweist. 
Die Zusammenfassung des JACCARD- und RENKONEN-Index zum WAINSTEINIndex und dessen Darstellung als Dendrogramm ergibt eine zusammenfassende Darstellung der biozönotischen Ähnlichkeit (Tab. 24, Abb. 118). Sehr deutlich wird die Außenseiterrolle von L1, die nicht nur zu den stark versauerten Probestellen gehört, sondern auch eine deutlich abweichende Choriotopstruktur aufweist. Die restlichen Probestellen teilen sich in zwei Gruppen auf. In der ersten Gruppe sind alle nicht versauerten Stellen und SC zu finden, in der zweiten Gruppe alle versauerten Stellen, wobei L2 und L3 eine gewisse Außenseiterposition in dieser Gruppe einnehmen, wodurch zusammen mit L1 der von den anderen Gewässern etwas abweichende eigenständige Charakter der Langen Bramke zum Ausdruck kommt. In der Gruppe der versauerten Probestelien heben sich zwei Untergruppen $a b$, von denen die erste sich aus den stark versauerten Probestellen M, S1 und B1 und die zweite sich aus den mäßig versauerten, durch häufige stärkere Säureschübe gekennzeichneten Stellen B2 und S2 zusammensetzt. Die Position von S3, L3 und L2 wird anhand der eigentlichen Indices deutlicher. Danach tendiert L2 mehr zu den stark versauerten und L3 zu den mäßig versauerten Stellen. S3 nimmt eine Mittelstellung zwischen den mäßig versauerten (S2, B2) und den schwach versauerten (SC, L3) Stellen ein.

Eine vergleichbare Analyse der Trichopteren-Zönosen erbrachte ähnliche Ergebnisse (RÜDDENKLAU 1989). Auch bei den Trichopteren kommt L1 eine Außenseiterposition zu. Darüber hinaus bilden bei dieser Tiergruppe B1 und B2 eine eigene Gruppe. Die restlichen Probestellen sind sehr deutlich in die nicht versauerten und die restlichen versauerten Probestellen aufgespalten, wobei auch hier zwischen $\mathrm{M}$ und $\mathrm{S} 1$ die Unähnlichkeit am geringsten ist. 


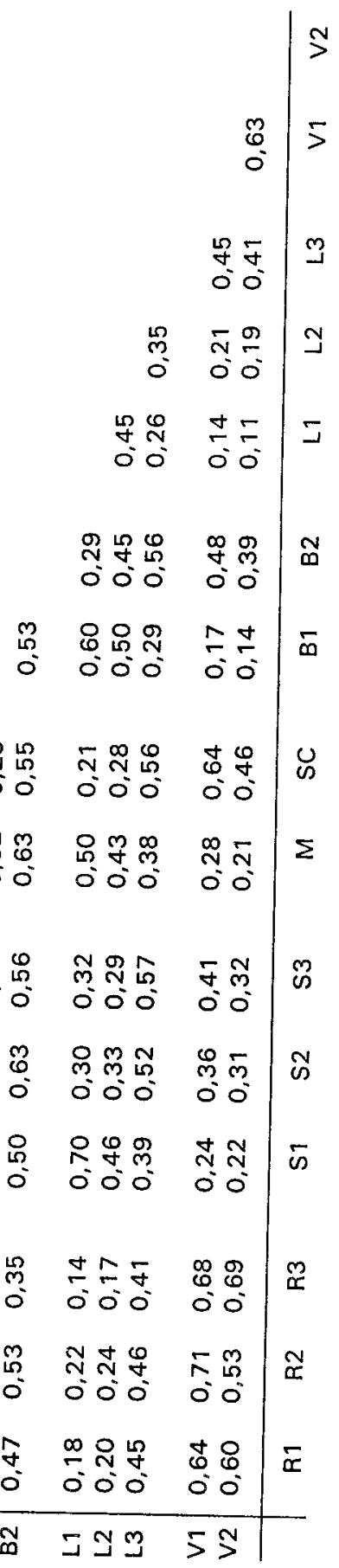




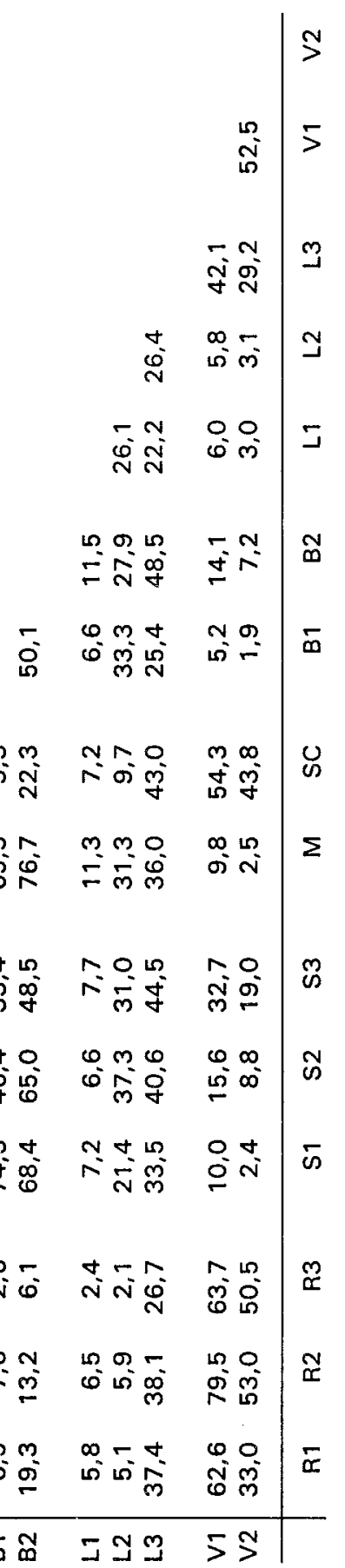




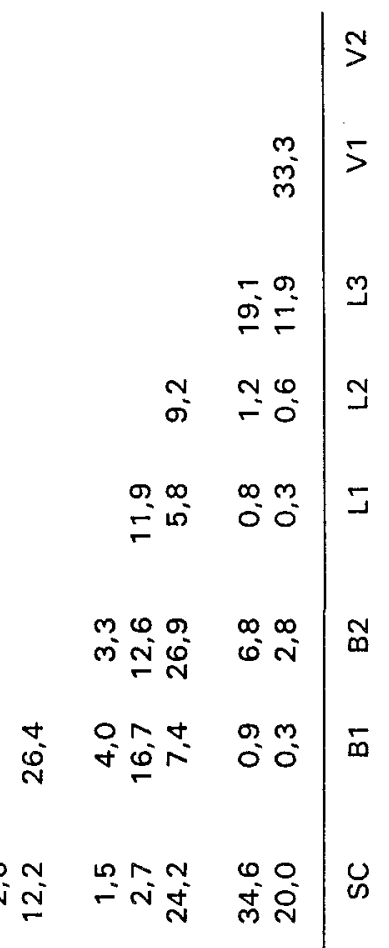

$$
\begin{aligned}
& \text { L }
\end{aligned}
$$

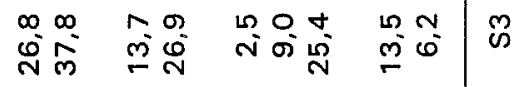

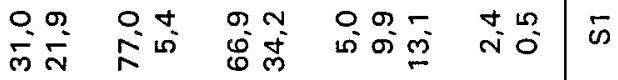

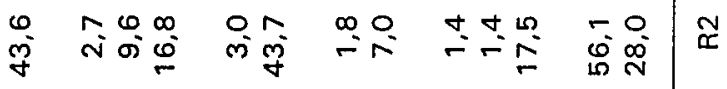

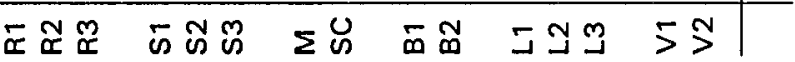

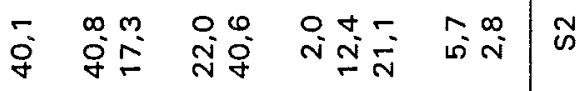

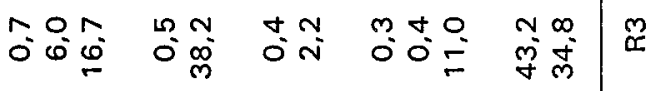

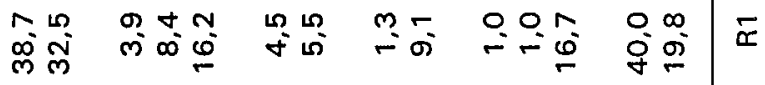




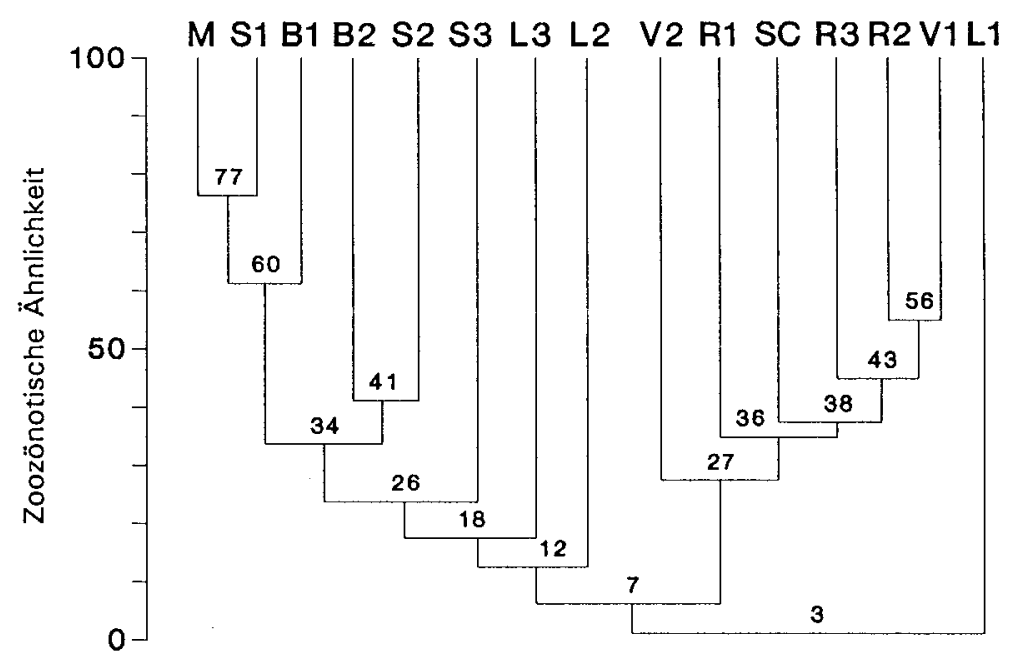

Abb. 118: Dendrogramm zur Cluster-Analyse der WAINSTEIN-Indices auf der Grundlage der Daten des gemeinsamen Emergenzerfassungszeit raums aller Probestellen 


\subsection{Ichthyozönosen}

Fische stellen ein inzwischen schon klassisches Objekt der ökologischen Forschung zur Gewässerversauerung dar. Die stark ausgeprägte Reaktion fast aller Arten auf die Erhöhung der Wasserstoffionenkonzentration und insbesondere auch die geringe Toleranz gegenüber der mit der Versauerung verbundenen starken Zunahme der für sie toxischen Aluminium-Spezies sowie die aufgrund der Langlebigkeit und Stellung in der Nahrungskette als Top-Prädatoren eine viel stärkere Bedeutung als bei den Invertebraten erlangende Akkumulation von Schwermetallen hat zu einer Vielzaht von fischereibiologischen, physiologischen und histologischen Untersuchungen an dieser Tiergruppe geführt (u.a. FROMM 1980, BAKER \& SCHOFIELD 1982, HOCH 1982, BROWN 1983, HENRIKSEN et al. 1984, LENHART \& STEINBERG 1984, KARLSSON-NORRGREN et al. 1986, MALTE 1986, FISCHER-SCHERL et al. 1988, NEVILLE \& CAMPBELL 1988, RAMAMOORTHY 1988, SEGNER et al. 1988).

\subsubsection{Vorkommende Arten}

In den Fließgewässern des Harzes können von Natur aus fast ausschließlich Bachforelle (Salmo trutta fario) und Groppe (Cottus gobio) angetroffen werden (REICHENBACH-KLINKE 1956, REICHENBACH-KLINKE 1958, LESSMANN \& HEITKAMP 1990). In einigen größeren Bächen sind darüber hinaus auch Elritze (Phoxinus phoxinus) und Bachschmerle (Noemacheilus barbatulus) $\mathrm{zu}$ finden (WÜSTEMANN 1989). Abgesehen von einer an $\vee 2$ im September 1986 gefangenen, aus dem Besatz des Granestausees stammenden Regenbogenforelle (Oncorhynchus mykiss) von $29,0 \mathrm{~cm}$ Länge und $278 \mathrm{~g}$ Gewicht konnten an den in die Untersuchungen einbezogenen Probestellen nur die beiden weit verbreiteten Arten festgestellt werden. Die groben Substratverhältnisse der untersuchten Gewässer müssen für Bachneunauge, Bachschmerle und Elritze als ungeeignet angesehen werden (GOLL 1985).

Die Verbreitung zeigt bereits eine sehr enge Beziehung zum Versauerungsgrad der Gewässer. Groppen wurden an vier Stellen gefangen, die alle in den nicht versauerten Bächen liegen. Als einzige der nicht versauerten Probestellen weist R1 keine Groppen auf. Bachforellen kommen an allen elf nicht, schwach oder mäßig versauerten Probestellen vor, wohingegen keine der stark versauerten Stellen einen Fischbestand hat. 
An allen untersuchten Gewässern liegt die Fischereiberechtigung bei den Forstverwaltungen, die die Bäche nicht bewirtschaften und insbesondere auch keinen Besatz durchführen, so daß von autochthonen Beständen ausgegangen werden kann. An Großer Söse nahe S2 und an Alter Riefensbeek $200 \mathrm{~m}$ oberhalb R3 befinden sich zwei kleinere mit Regenbogenforellen besetzte Fischteiche, die aus den Bächen gespeist werden. Aus diesen Teichanlagen konnte zu keinem Zeitpunkt ein Tier im Bach selbst gefangen werden.

Die Groppe ist ein stationär lebender, bodenorientierter und dämmerungsaktiver Fisch, der in kühlen sauerstoffreichen Fließgewässern mit steinigem Grund lebt (GAUMERT 1986, GOLL 1985). Sie ernährt sich überwiegend von größeren Arten des Makrozoobenthos (WELTON et al. 1983). Die Laichzeit liegt zwischen März und Mai, die Entwicklungsdauer der Eier beträgt 4 - 6 Wochen und die Tiere sind am Ende des zweiten Lebensjahres geschlechtsreif. Groppen reagieren sehr empfindlich auf Verschlechterungen der Wasserqualität und Biotopveränderungen (GOLL 1985, SPÄH \& BEISENHERZ 1986, GAUMERT 1986). Die Art ist in besonderem Maße durch die Gewässerversauerung in ihrem Bestand gefährdet (STEINBERG 1983, LUBIENIECKI \& STEINBERG 1985, LESSMANN \& HEITKAMP 1990).

Die Bachforelle kommt als Charakterart im Rhithral von Bächen und Flüssen stationär vor. Die Nahrung besteht bei den jüngeren Tieren vor allem aus Benthosorganismen und Anflug, bei älteren auch aus größeren Beutetieren wie zum Beispiel Kleinfischen. Die Laichzeit erstreckt sich mit regionalen Unterschieden auf die Wintermonate zwischen November und März. Die Entwicklungsdauer beträgt 410 Tagesgrade. Die Männchen sind im zweiten, die Weibchen im dritten Jahr geschlechtsreif. Gefährdet ist die Art vor allem durch übermäßige Schadstoffbelastungen, Gewässerausbau und unnötigen Besatz (GAUMERT 1981, SCHINDLER 1975).

\subsubsection{Groppenpopulationen}

Wegen der Längenselektivität der Elektrobefischung können die tatsächlichen Besiedlungsdichten der Groppe nur angenähert ermittelt werden. Die gewonnenen Daten vermitteln dennoch einige wichtige Ergebnisse für einen relativen Vergleich. In der Alten Riefensbeek liegen die Besiedlungsdichten im Mittel der fünf Befischungen an R3 um etwa 200 Ind./ha höher als an R2, wobei zwischen 


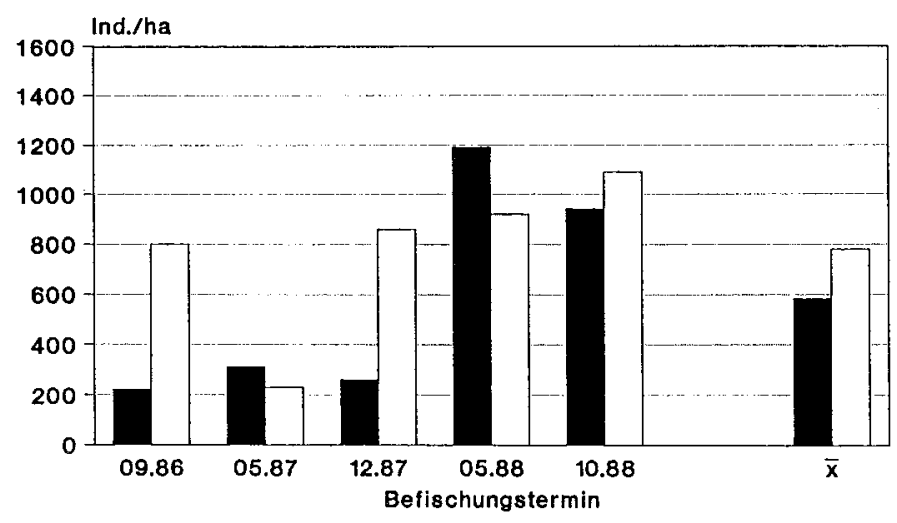

R2 $\square$ R3

Abb. 119: Groppen-Dichten in der Alten Riefensbeek

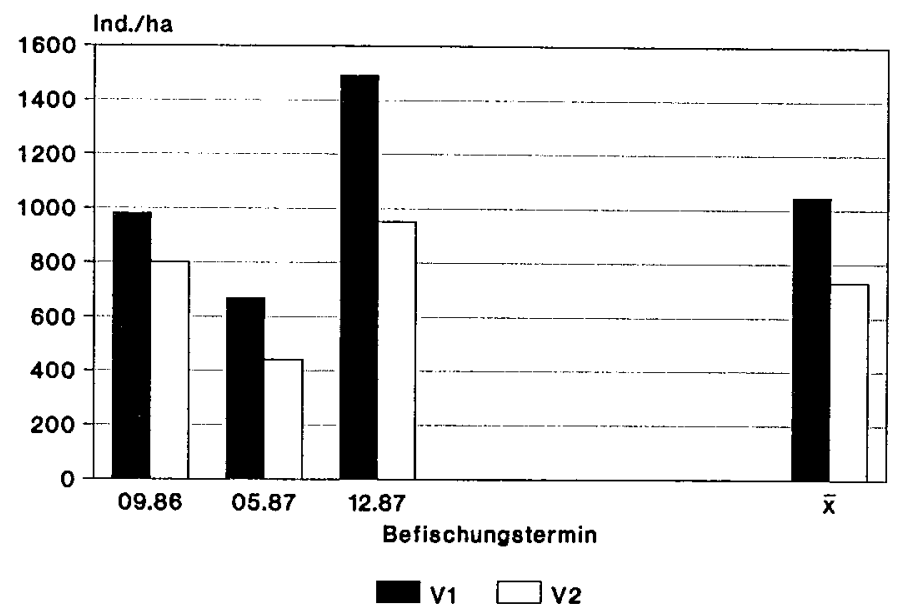

Abb. 120: Groppen-Dichten im Varleybach 
den einzelnen Terminen sehr starke Schwankungen festzustellen sind (Abb. 119). Im Varleybach treten die Unterschiede etwas schwächer auf, sind aber dennoch sehr groß. Im Gegensatz zur Alten Riefensbeek mit einer Zunahme der mittleren Besiedlungsdichte flußabwärts, konnten im Varleybach an V1 stets deutlich höhere Dichten festgestellt werden als an V2 (Abb. 120). Auffällig ist in beiden Gewässern der Rückgang zwischen September 1986 und Mai 1987. Dazwischen lag ein relativ kalter Winter. Bis Dezember 1987 haben sich die Populationen wieder erholt, und der folgende sehr milde Winter trägt, wie in der Alten Riefensbeek festgestellt werden kann, zu einem weiteren Anwachsen der Besiedlungsdichte bei. Im Vergleich mit Untersuchungen an der Sieber (maximal 180 Ind./hal konnten relativ hohe Besiedlungsdichten festgestellt werden (LESSMANN 1983, HEITKAMP et al. 1985). Daß die Dichten dennoch als niedrig einzustufen sind, machen die in der Hase bei Osnabrück ermittelten 8500 ind./ha deutlich (ZUCCHI \& GOLL 1981).

Der im Vergleich von den anderen drei Probestellen abweichende Verlauf der Groppenabundanzen an R2 spricht für eine erst relativ junge Besiedlung oder Wiederbesiedlung dieser Stelle, die im Bereich der oberen Verbreitungsgrenze der Groppe in der Alten Riefensbeek liegt. Da Groppen im Brunnenbach bis zu einer Höhe von $600 \mathrm{~m}$ in größerer Dichte vorkommen (LESSMANN \& HEITKAMP 1990), kann das Fehlen der Art an R1 nicht durch ein Überschreiten einer Höhenverbreitungsgrenze erklärt werden. An R3 konnten wie an den Stellen des Varleybachs zu allen Befischungsterminen alle Altersklassen angetroffen werden. Im Gegensatz dazu wurden an R2 in den beiden ersten Untersuchungsjahren nur wenige größere Tiere gefangen. Erst 1988 hatte sich eine mit den anderen Stellen vergleichbare Population mit Eier tragenden Weibchen und Jungtieren aufgebaut. So betrug die mittlere Länge der an R2 gefangenen Tiere im September $19869,2 \mathrm{~cm}$, im Gegensatz zu $5,5 \mathrm{~cm}$ an R3, und hatte im Oktober 1988 mit $5,2 \mathrm{~cm}$ einen nahezu identischen Wert mit R3, wo $5,4 \mathrm{~cm}$ ermittelt wurden.

\subsubsection{Bachforellenpopulationen der Hauptuntersuchungsbäche}

Bei der Bachforelle spiegeln nicht nur Verbreitung sondern auch Altersaufbau der Populationen, Besiedlungsdichte und Biomasse die starke Abhängigkeit der Art von unversauerten Gewässern wider. 


\subsubsection{Populationsstruktur}

Die Betrachtung der Längenzusammensetzungen zeigt gravierende Störungen des Altersaufbaus der Populationen an den versauerten Probestellen (Abb. 121 . 125). Im Vergleich der beiden Hauptuntersuchungsbäche konnten in der Alten Riefensbeek an allen Stellen zu allen Befischungsterminen natürliche Populationen angetroffen werden. Aufgrund des strengen Winters 1986/87 mit entsprechend langer Entwicklungsdauer der Forelleneier ließen sich im Mai 1987 in den Bächen noch keine Tiere der Altersklasse $0+$ nachweisen. Im Dezember war dieser Jahrgang jedoch an allen Probestellen der Alten Riefensbeek vorhanden. Nach dem milden Winter $1987 / 88$ konnten bereits im Mai 1988 Tiere der Altersklasse $\mathrm{O}+$ an R1 und R3 gefangen werden. Bis zum Herbst trat dieser Jahrgang wieder deutlich an allen Probestellen auf. Stets stellen die Altersklassen $1+$ und $2+$ den weitaus größten Teil der Populationen, noch ältere Tiere kommen nur vereinzelt vor.

In der Großen Söse fehlte die Altersklasse O+ im Mai 1987 an Probestelle S3 wie an allen Probestellen der Alten Riefensbeek. Aber auch im Dezember 1987 bestand die Bachforellenpopulation nur aus Tieren der Altersklassen $\geq 1+$, so daß es in diesem Jahr zu keiner Reproduktion kam. 1988 konnten sowohl im Mai als auch im Oktober Tiere der Altersklasse $\mathrm{O}_{+}$in geringer Anzahl gefangen werden, wobei im Oktober aber nur $58 \%$ der Stärke des an R3 zu verzeichnenden Jahrgangs erreicht wurden. Entscheidender Unterschied zwischen beiden Jahren waren die Intensitäten der Winter mit viel Schnee im strengen Winter $1986 / 87$ und lange Zeit keinem Schnee im milden Winter 1987/88, so daß es auch zu keinen größeren Schneeschmelzen mit entsprechenden längeren Säureschüben in diesem Winter kam. Ende Dezember 1986 trat hingegen ein extremes Hochwasser auf.

An S2, wo die Bachforellen an einem für sie unüberwindbaren Sohlsturz ihre Verbreitungsgrenze in der Großen Söse haben, bestand die Population zu allen Befischungsterminen ausschließlich aus sehr wenigen stets um die $20 \mathrm{~cm}$ großen, mehrjährigen Tieren. 
Abb. 121: Längenzusammensetzung der Bachforellenpopulation zu den einzelnen Befischungsterminen an R1
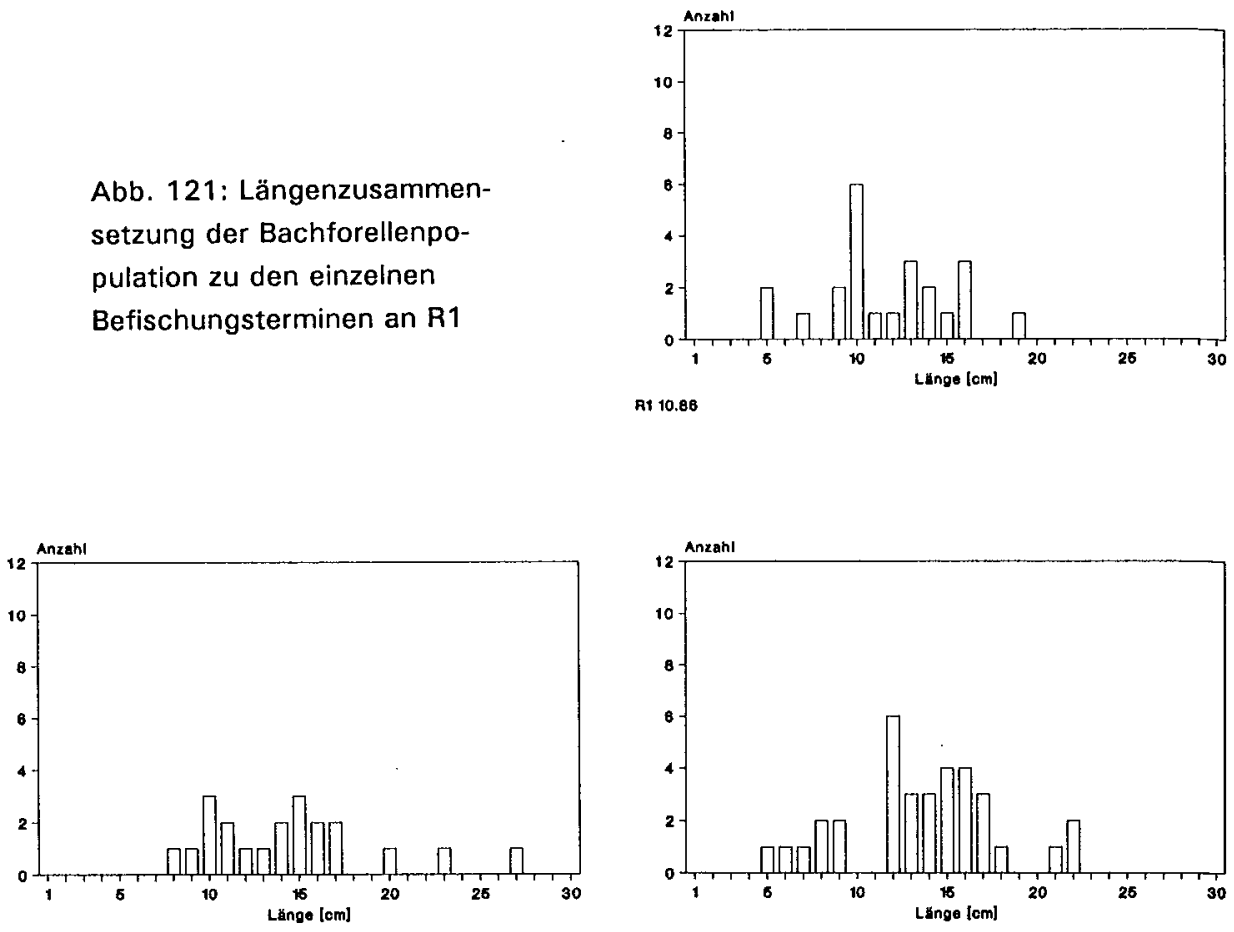

ค1 05.87

A1 12.87
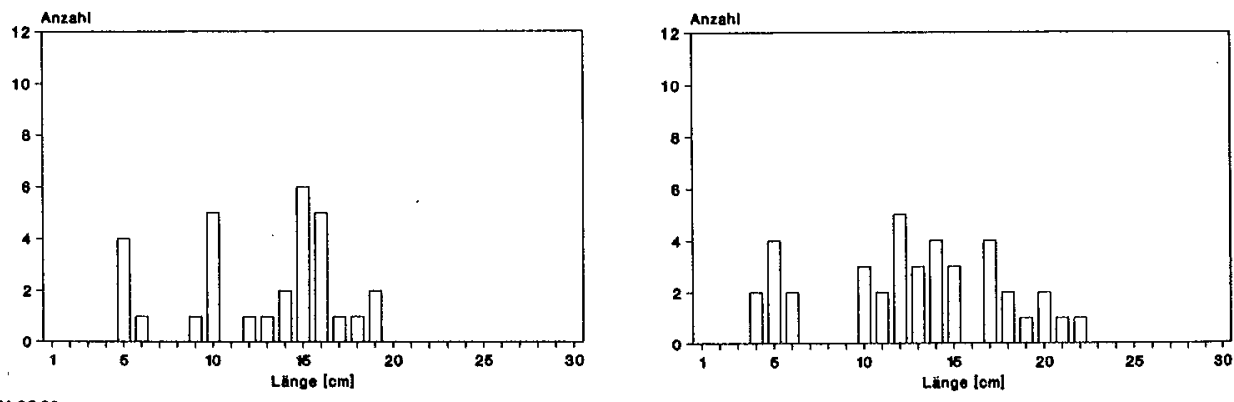

R1 06.88

A1 10.88 
Abb. 122: Längenzusammensetzung der Bachforellenpopulation zu den einzelnen Befischungsterminen an R2

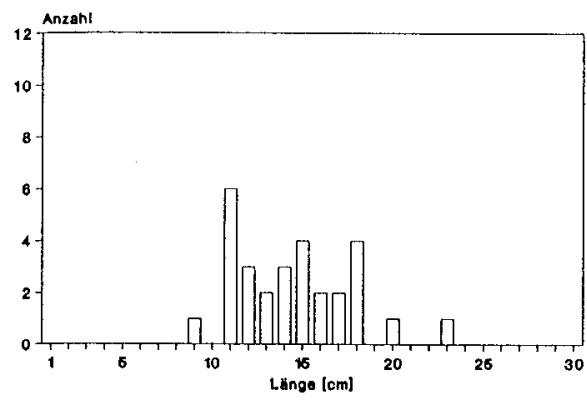

F2 06.87

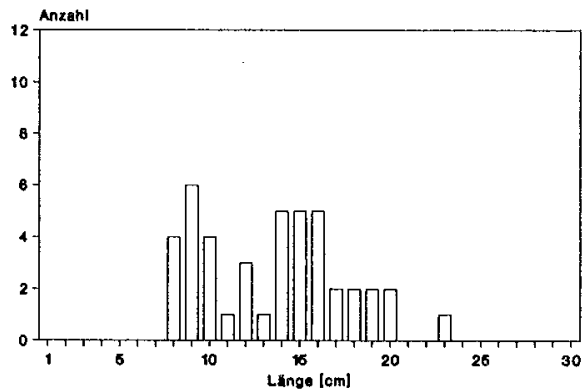

F2 06.88

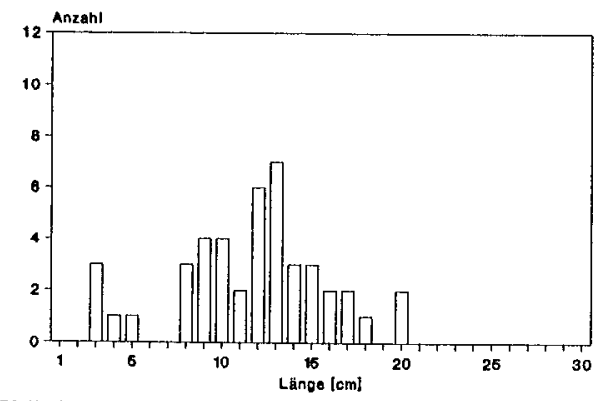

R2 10.86

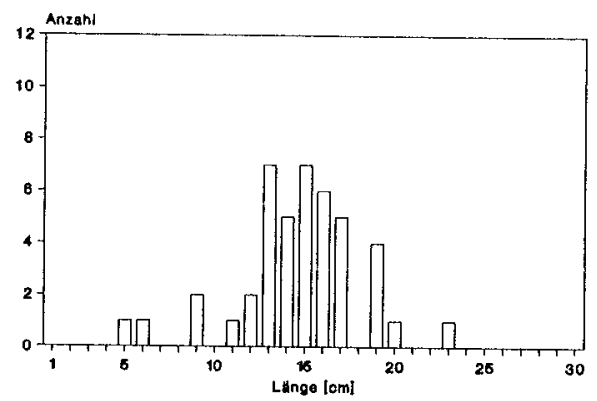

R2 12.67

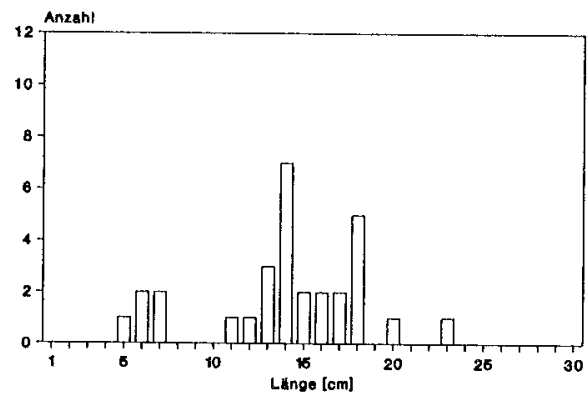

A2 10.88 
Abb. 123: Längenzusammensetzung der Bachforellenpopulation zu den einzelnen Befischungsterminen an R3

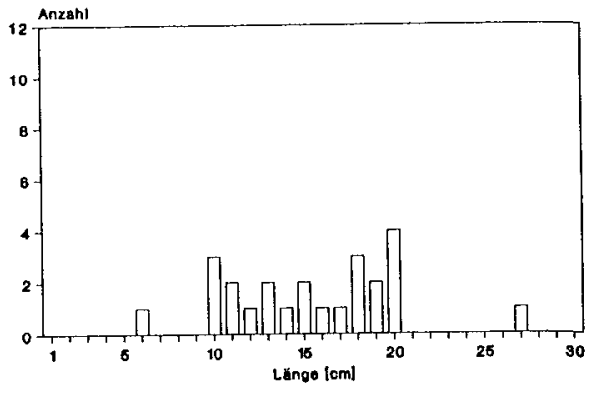

A3 10.86
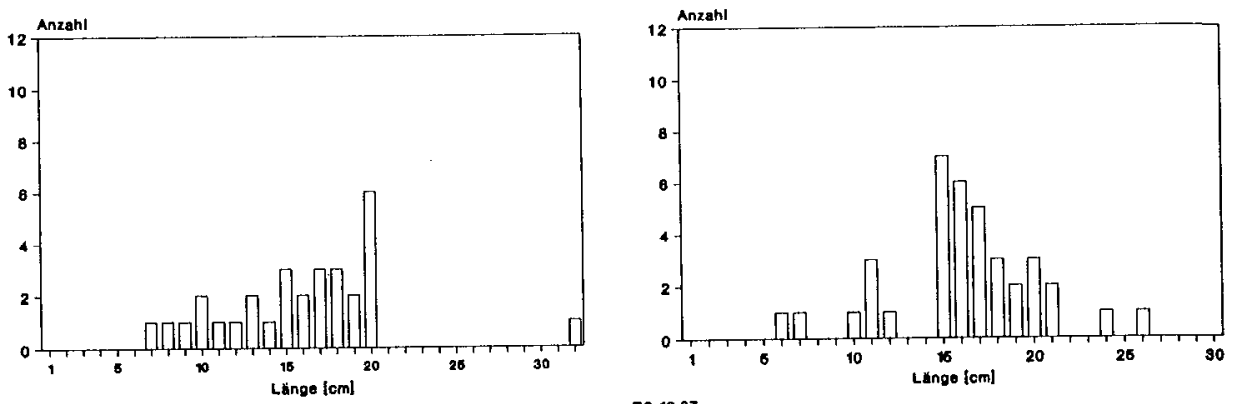

R3 06.87

F3 12.87
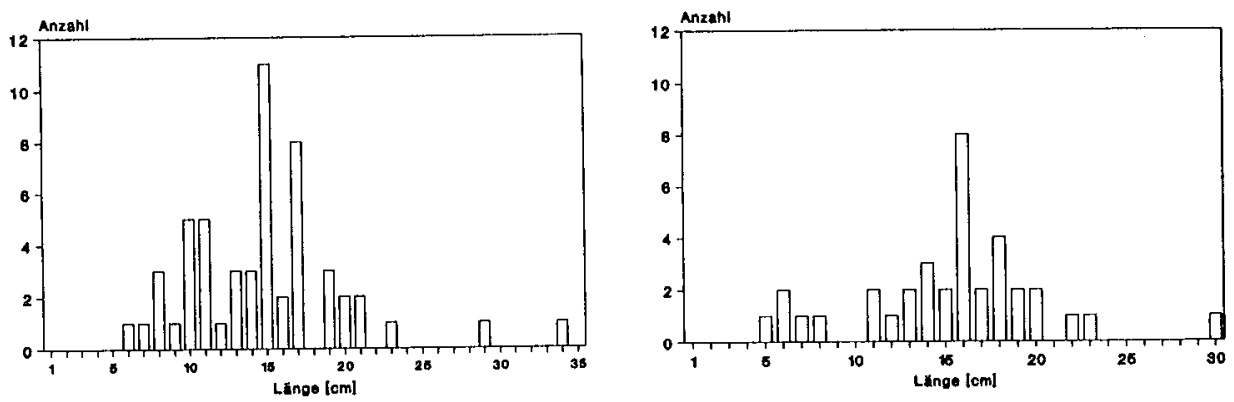

ค3 08.88

ค3 10.88 
Abb. 124: Längenzusammensetzung der Bachforellenpopulation zu den einzelnen Befischungsterminen an S2

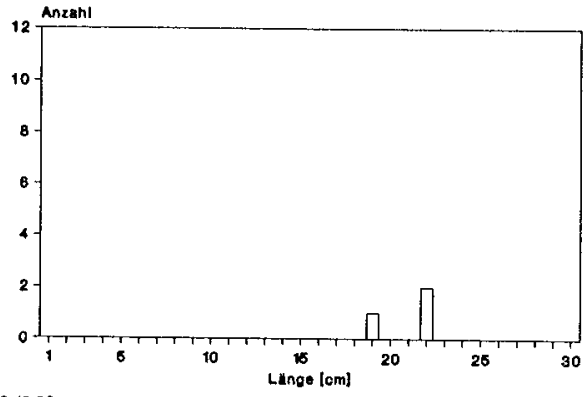

8210.86
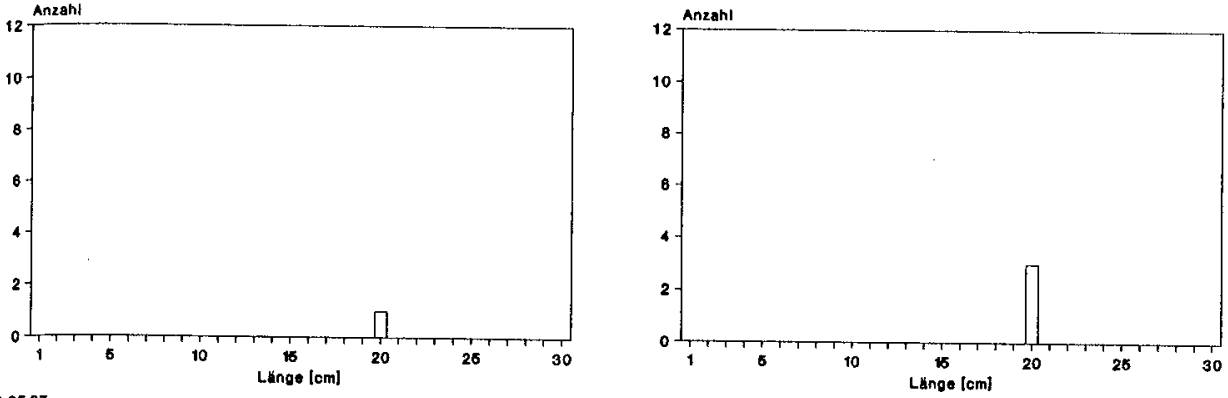

$\$ 205.87$

S2 12.87
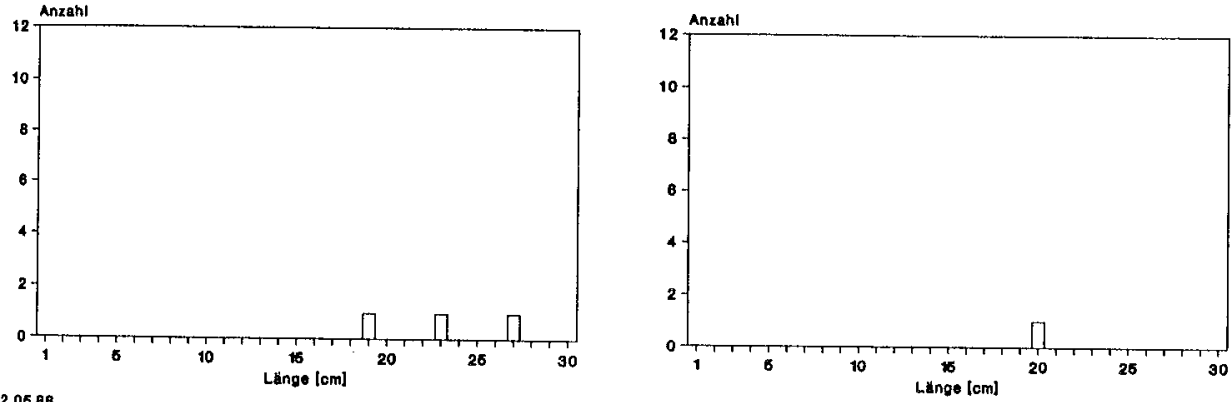

8210.88 
Abb. 125: Längenzusammensetzung der Bachforellenpopulation zu den einzelnen Befischungsterminen an \$3

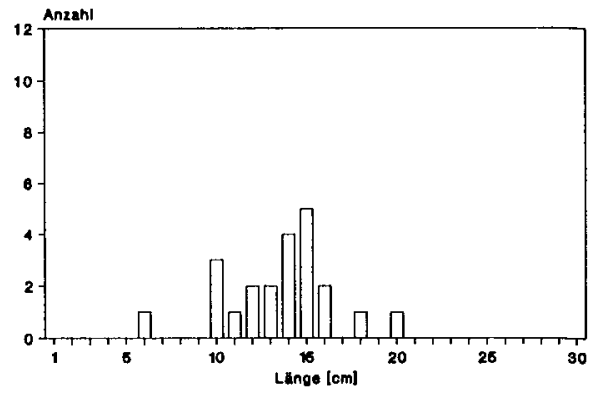

8310.88
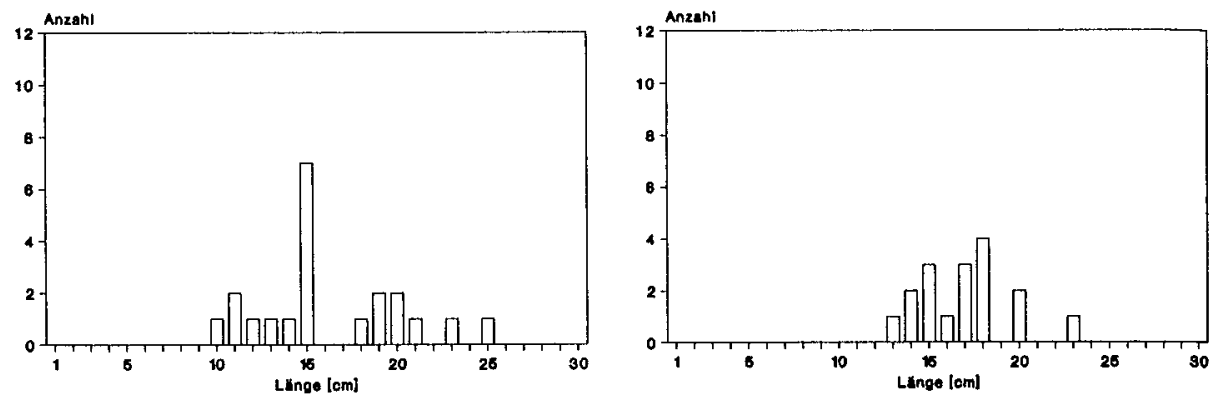

8306.87

8312.87
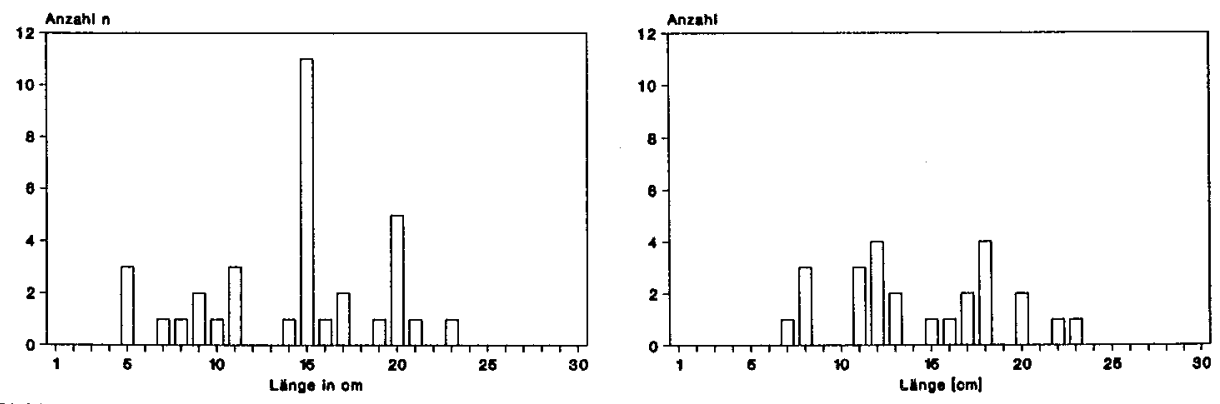

S3 05.88

8310.88 


\subsubsection{Besiedlungsdichte}

Im Vergleich der fünfmaligen Befischungen an den beiden Hauptuntersuchungsbächen zeichnet sich eine stark ausgeprägte Abnahme der Besiedlungsdichten von R1 nach R3 ab, die abgesehen vom Mai 1988 auch zu allen einzelnen Befischungsterminen festzustellen war (Abb. 126, 127). Im Gegensatz dazu ist in der Großen Söse nach dem völligen Fehlen der Bachforelle an S1 ein nur sehr kleiner Bestand an der mäßig versauerten Stelle S2 vorhanden, der im Mittel nur $7 \%$ von R2 erreicht. Im schwach sauren Bereich an S3 ist der Bachforellenbestand auf etwa $50 \%$ von R3 angewachsen. An beiden unteren Stellen wurden im Mai 1988 nach dem relativ milden Winter die, höchsten Dichten aller Befischungstermine registriert. An R3 hatte sich seit September 1986 mehr als eine Verdoppelung des Bestandes ergeben. Bis zum Oktober 1988 nahmen die Dichten an beiden Stellen jedoch wieder stark ab. An R1, R2 und S2 waren die Unterschiede zwischen den einzelnen Terminen uneinheitlich und relativ gering.

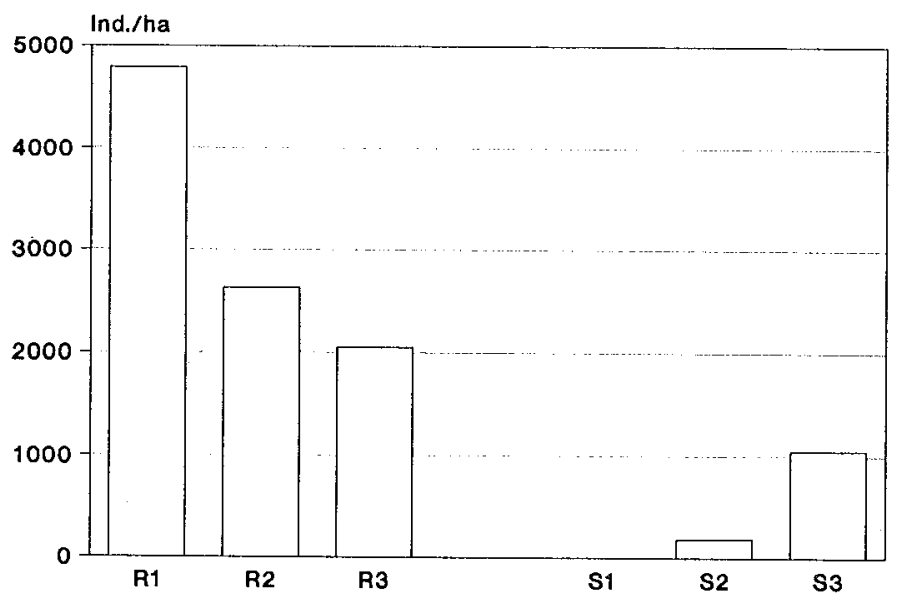

Abb. 126: Mittlere Bachforellenbesiedlungsdichten an den Probestellen von Alter Riefensbeek und Großer Söse 


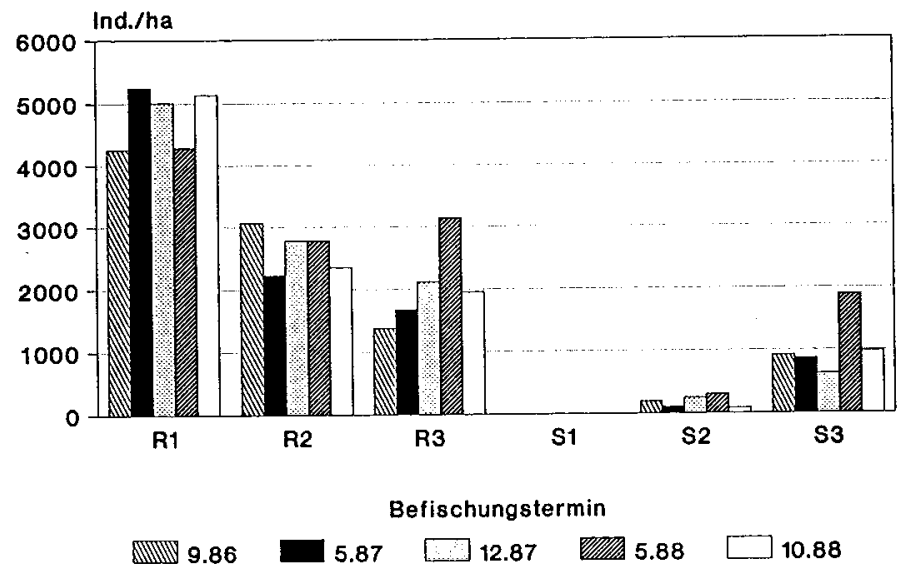

Abb. 127: Besiedlungsdichten der Bachforelle zu den einzelnen Befischungsterminen an den Probestellen von Alter Riefensbeek und Großer Söse

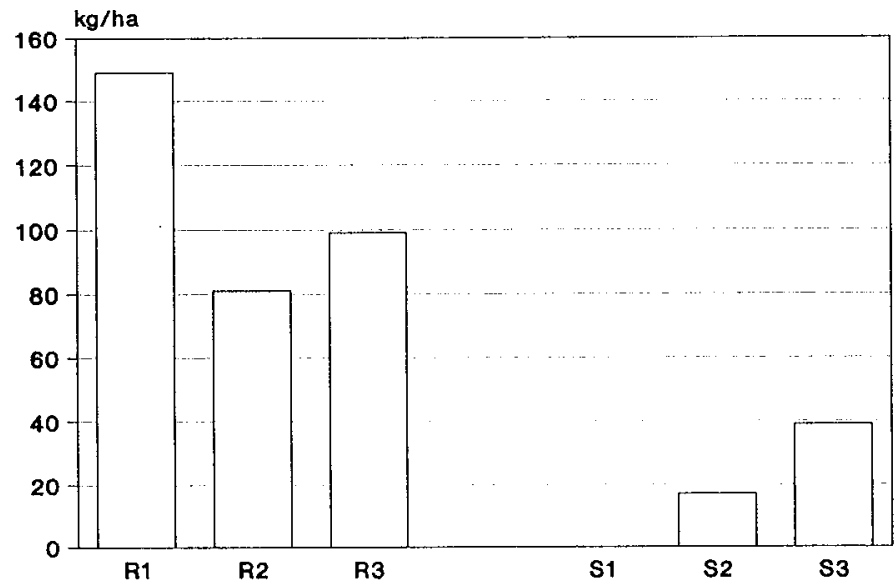

Abb. 128: Mittlere Biomassen der Bachforellen an den Probestellen von Alter Riefensbeek und Großer Söse 


\subsubsection{Biomasse}

Für die mittlere Biomasse ergeben sich den Besiedlungsdichten entsprechende, wesentlich höhere Werte in der Alten Riefensbeek als in der Großen Söse (Abb. 128). S2 erreicht nur $21 \%$ von R2 und S3 $39 \%$ von R3. Der BiomassenAnstieg von R2 nach R3 erklärt sich aus dem bachabwärts zunehmenden Anteil größerer und schwererer Tiere. Dies ist auch der Grund für den relativ hohen Wert an S2, wo ausschließlich größere Bachforellen gefangen wurden.

\subsubsection{Korpulenzfaktor}

Der über den Ernährungszustand der Fische Auskunft gebende Korpulenzfaktor zeigt mit einem Mittelwert um 1,0 für alle Probestellen bei nur leicht höheren Werten an der Alten Riefensbeek keine gravierenden Unterschiede zwischen beiden Bächen an (Abb. 129, 130). Die großen Unterschiede an S2 erklären sich vor allem aus der natürlichen Streuung aufgrund der sehr geringen Individuenzahl. Relativ große Schwankungen sind auch an R2 und R3 mit über dem Durchschnitt liegenden Werten vor allem an den ersten beiden Befischungsterminen zu beobachten. Eine Beziehung zur Besiedlungsdichte besteht nicht. Auch konnten in dem betroffenen Zeitraum keine ungewöhnlich hohen Benthosdichten oder Emergenzraten festgestellt werden, die auf ein größeres Nahrungsangebot hätten schließen lassen.

5.6.4 Bachforellenpopulationen der Haupt- und Nebenuntersuchungsbäche im Vergleich

\subsubsection{Populationsstruktur}

Die an den Nebenuntersuchungsbächen gewonnenen Ergebnisse bestätigen die starke Abhängigkeit der Bachforellen-Populationsstrukturen von der Gewässerversauerung. Wie an $\$ 2$ kommen mit B2 an einer zweiten mäßig versauerten Probestellen ausschließlich sehr wenige große, ältere Tiere vor (Abb. 131). An L2 stellt sich der Populationsaufbau dagegen noch günstiger dar, weist aber bereits auf eine dramatische Entwicklung hin (Abb. 132). Direkt unterhalb der Probestelle befindet sich seit Ende der 40er Jahre ein Meßwehr, das für aufwandernde Fische unpassierbar ist. Die oberhalb des Wehres lebende Bachforellenpopulation ist seitdem isoliert. Es konnten hier nur wenige Tiere, die 


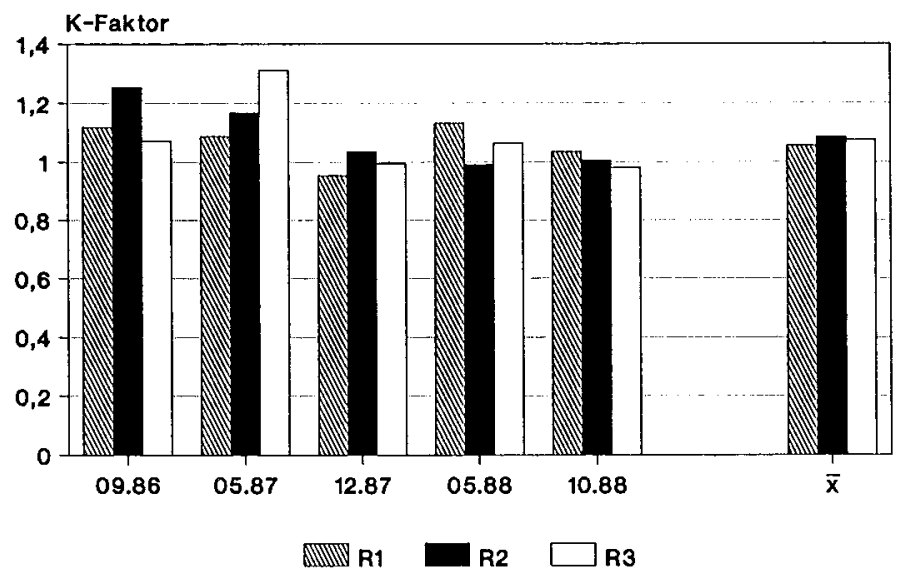

Abb. 129: Korpulenzfaktoren der Bachforellenpopulationen zu den einzelnen Befischungsterminen an den Probestellen der Alten Riefensbeek

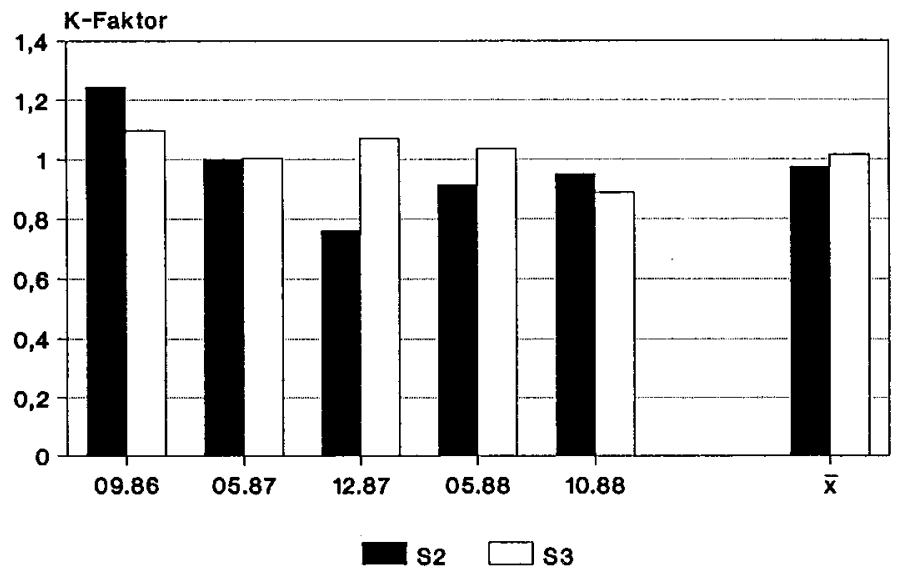

Abb. 130: Korpulenzfaktoren der Bachforellenpopulationen zu den einzelnen Befischungsterminen an den Probestellen der Großen Söse 
alle größer waren als $10 \mathrm{~cm}$, gefangen werden. Mit einem Erlöschen der Population muß daher bei einem Fortschreiten der Gewässerversauerung in den nächsten Jahren gerechnet werden. Auch unterhalb des Wehres fehlen Jungforellen des Jahrgangs $0+$ bereits vollständig. Ältere Jahrgänge sind jedoch in weitestgehend normaler Zusammensetzung vorhanden, und im Dezember 1987 wurde dort auch Laich tragende Weibchen gefangen.

Von den schwach versauerten Probestellen ähnelt die Populationsstruktur an SC weitestgehend der an L2, wobei hier sogar überwiegend noch größere Tiere vorkommen (Abb. 133). Eine Reproduktion findet im untersuchten Bereich der Großen Schacht nicht statt. Im Gegensatz dazu sind an der am schwächsten versauerten Stelle L3 (Abb. 134) und an den nicht versauerten Stellen des Varleybachs (Abb. 135, 136), wie schon an den Probestellen der Alten Riefensbeek beobachtet, zu jedem Befischungstermin alle Jahrgänge zu finden. Die Zahl der gefangenen Jungforellen ist aber an allen Stellen größeren Schwankungen von Befischungstermin zu Befischungstermin unterworfen. Die Altersklasse $\mathrm{O}_{+}$ erreicht an V2 die höchste Anzahl.

Damit sind die nicht versauerten Probestellen und L.3 die einzigen Stellen der Untersuchungen, an denen in jedem der erfaßten Jahre eine Reproduktion stattgefunden hat. Von einer in Einzeljahren möglichen Vermehrung kann man noch an S3 ausgehen. An L2 und SC kommen zwar noch die Altersklassen > zahlreich vor, eine erfolgreiche Reproduktion konnte jedoch nicht festgestellt werden. Nur ein zeitweiliges Überleben ist Bachforellen an S2 und B2 möglich, die ausschließlich von sehr wenigen großen, älteren Tieren besiedelt werden.

\subsubsection{Besiedlungsdichte und Biomasse}

Besiedlungsdichte und Biomasse zeigen für die gemeinsamen Untersuchungen an allen 15 Stellen, den Populationsstrukturen entsprechend, die höchsten Werte für die nicht versauerten Gewässer und $L 3$ an (Abb. 137, 138). Im Varleybach nimmt wie in der Alten Riefensbeek die Zahl der größeren Tiere bachabwärts $z u$, so daß die Unterschiede in der Besiedlungsdichte bei der Biomasse fast ausgeglichen werden. Die sehr hohen Werte für L2 erklären sich aus der besonderen Struktur des abgefischten Bachabschnitts, der bei relativ großer Wasserführung nur eine durchschnittliche Breite von $0,5 \mathrm{~m}$ hat und zudem unterhalb eines für Fische nicht passierbaren Wehres liegt, wodurch es 
zu einem "Staueffekt" aufwanderungswilliger Tiere kommen kann. Die Werte sind deshalb mit den anderen nur eingeschränkt vergleichbar. Sehr deutlich setzen sich die restlichen versauerten Probestellen dagegen mit ihren sehr niedrigen Werten ab.

Die Auftragung der mittleren Längen und des mittleren Gewichts der gefangenen Bachforellen ( $\mathrm{Abb}$. 139, 140) stellt zum einen noch einmal die für die Reproduktion wichtigen Stellen heraus, macht aber zum anderen auch deutlich, daß sich bachabwärts die Unterschiede bei einem allgemein höheren Anteil größerer Tiere zunehmend verwischen, solange die mittleren $\mathrm{pH}$-Werte bei oder über 6,0 liegen. Der Unterschied zu-den Stellen mit den mittleren $\mathrm{pH}$-Werten unter 6,0 tritt deutlich hervor, genauso wie das Fehlen der Bachforelle bei mittleren $\mathrm{pH}$ Werten unter 5,0 .

\subsubsection{Korpulenzfaktor}

Wie bereits bei den Hauptuntersuchungsbächen ergeben sich auch bei den Bächen der Nebenuntersuchungen keine Beziehungen des Ernährungszustandes der Fische zur Gewässerversauerung. Der Korpulenzfaktor liegt an fast allen Stellen zwischen 1,0 und 1,1 und erreicht nur im Varleybach etwas höhere Werte (Abb. 141). Da die versauerten Gewässerbereiche mit Fischvorkommen nicht nur niedrigere Benthosbesiedlungsdichten aufweisen sondern auch einen geringeren Fischbestand, kommt es zu keiner Verschlechterung der Ernährungssituation für den einzelnen Fisch. Aus dem Gesamtplot von Gewicht und Länge sind keine größeren Abweichungen einzelner Probestellen oder einzelner Tiere zu entnehmen (Abb. 142). Die größten gefangenen Fische mit Längen über $30 \mathrm{~cm}$ stammen von $\mathrm{R} 3$ und insbesondere $\mathrm{V} 2$. 
Abb. 131: Längenzusammensetzung der Bachforellenpopulation zu den einzelnen Befischungsterminen an B2

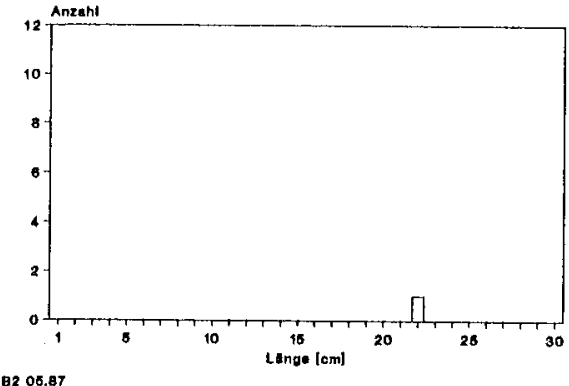

Abb. 132: Längenzusammensetzung der Bachforellenpopulation zu den einzelnen Befischungsterminen an L2

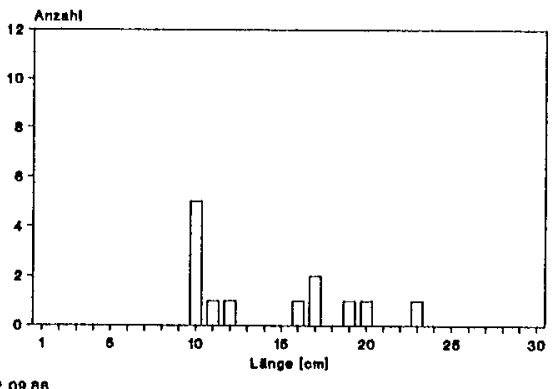
L2 09.8B
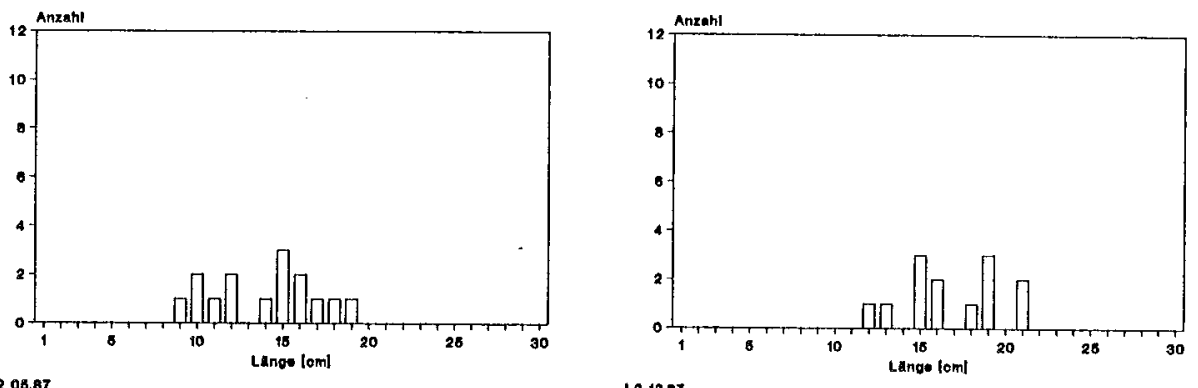
Abb. 133: Längenzusammensetzung der Bachforellenpopulation zu den einzelnen Befischungsterminen an SC

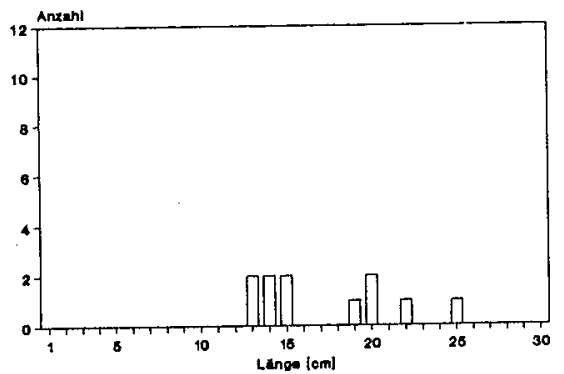
sc 05.87

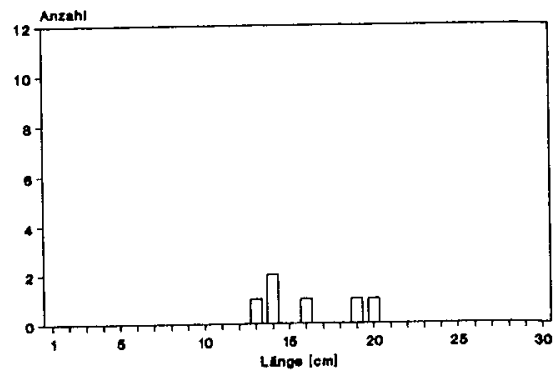

$8 \mathrm{sc} 00,86$

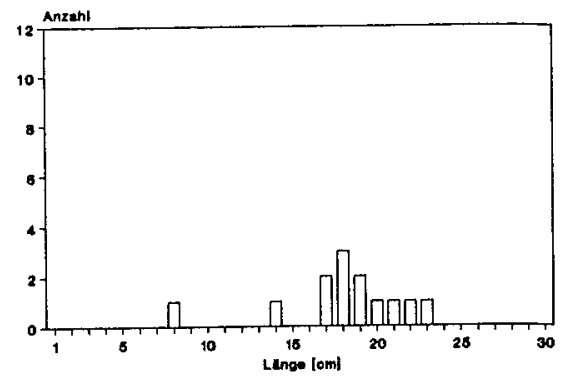

$\operatorname{sc} 12,87$

Abb. 134: Längenzusammensetzung der Bachforellenpopulation zu den einzelnen Befischungsterminen an L3
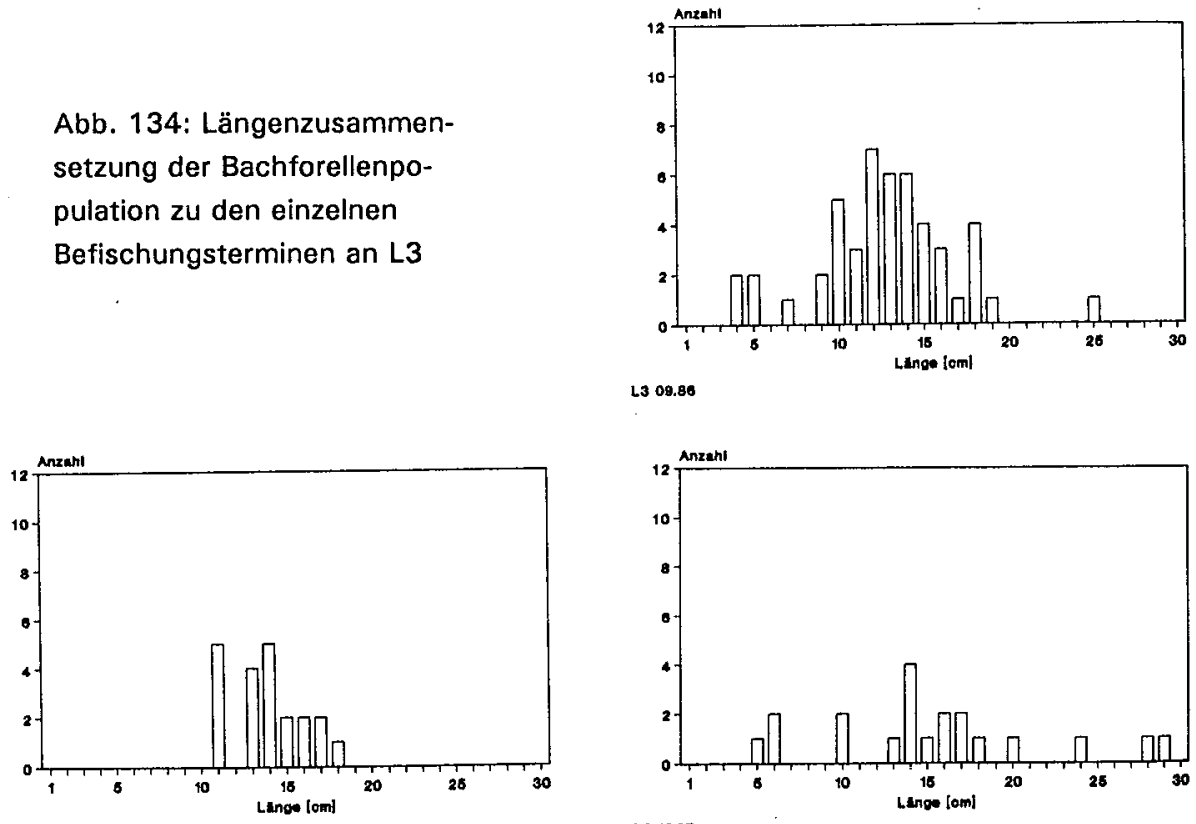

L3 09.86

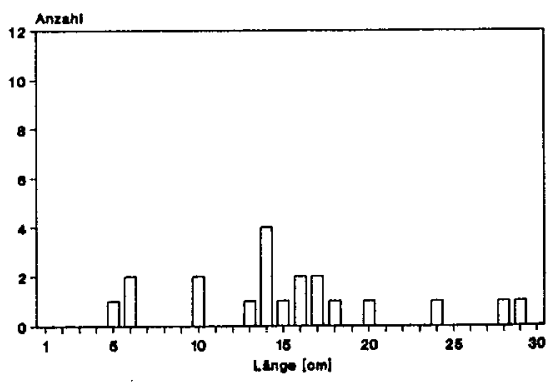

L3 12.8T 
Abb. 135: Längenzusammensetzung der Bachforellenpopulation zu den einzelnen Befischungsterminen an $\mathrm{V} 1$

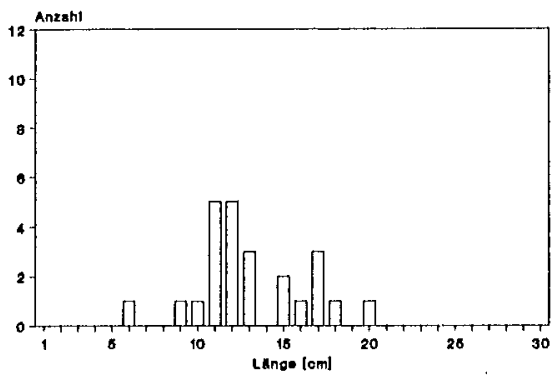

vios.e7

Abb. 136: Längenzusammensetzung der Bachforellenpopulation zu den einzelnen Befischungsterminen an V2

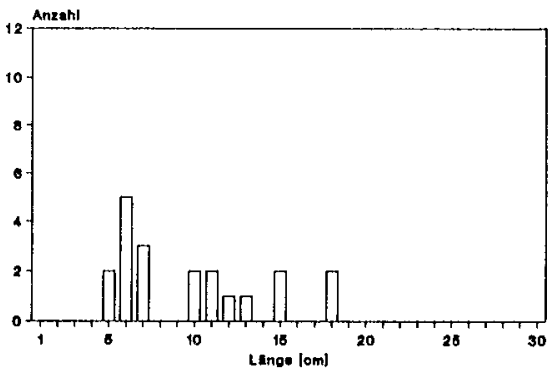
V2 08.87

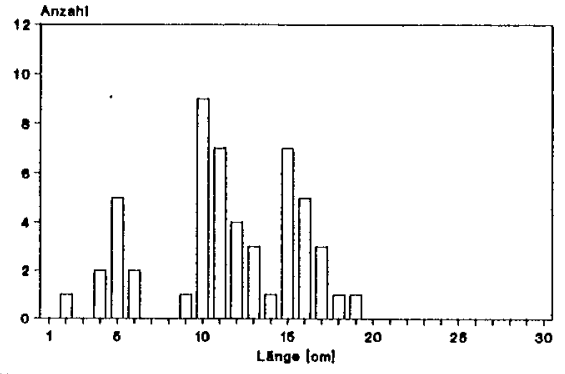

v1 09.88

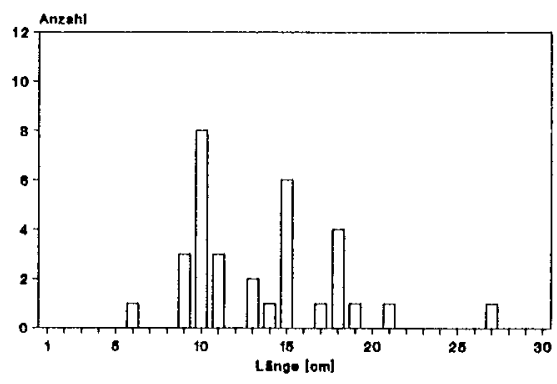

VI 12,87

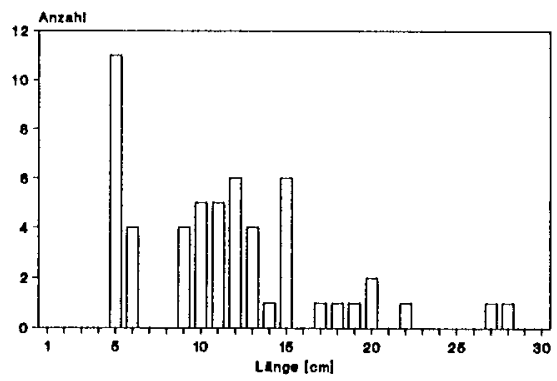

v2 09.00

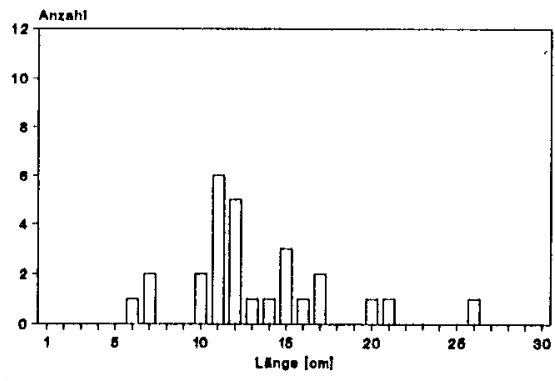

V2 12.87 


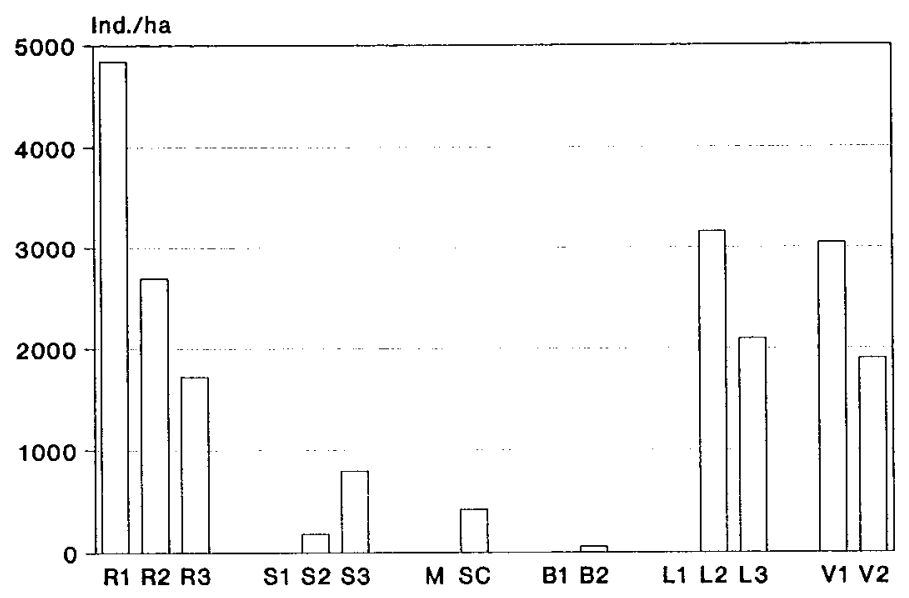

Abb. 137: Mittlere Bachforellen-Besiedlungsdichten der gemeinsamen Befischungstermine

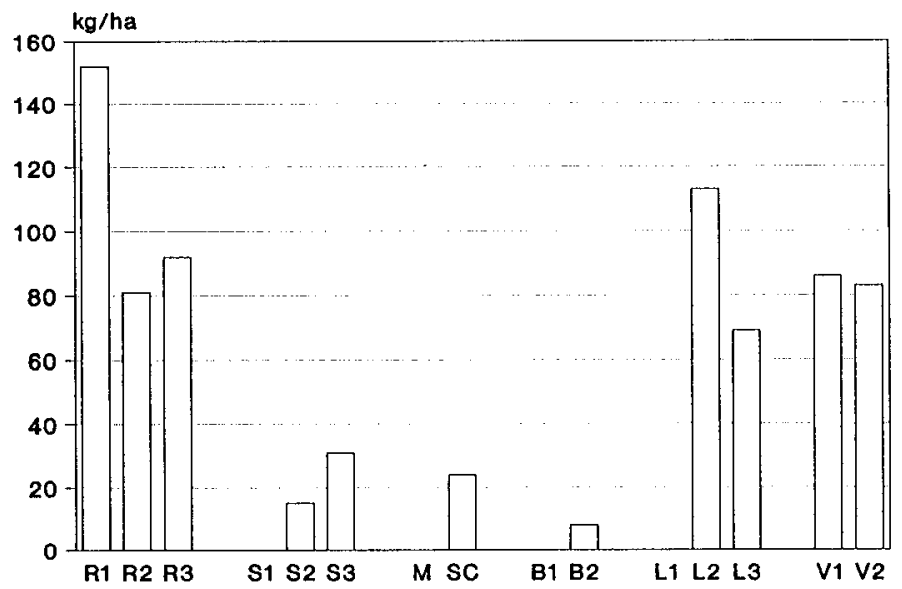

Abb. 138: Mittlere Bachforellen-Biomassen der gemeinsamen Befischungstermine 


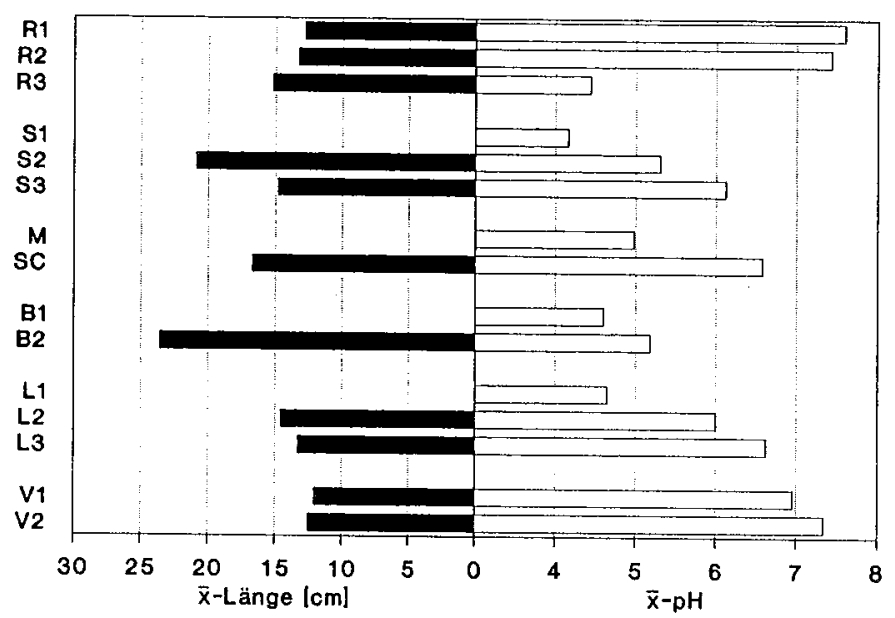

Abb. 139: Vergleich zwischen mittlerer Länge der Bachforellen und den mittleren $\mathrm{pH}$-Werten der Probestellen

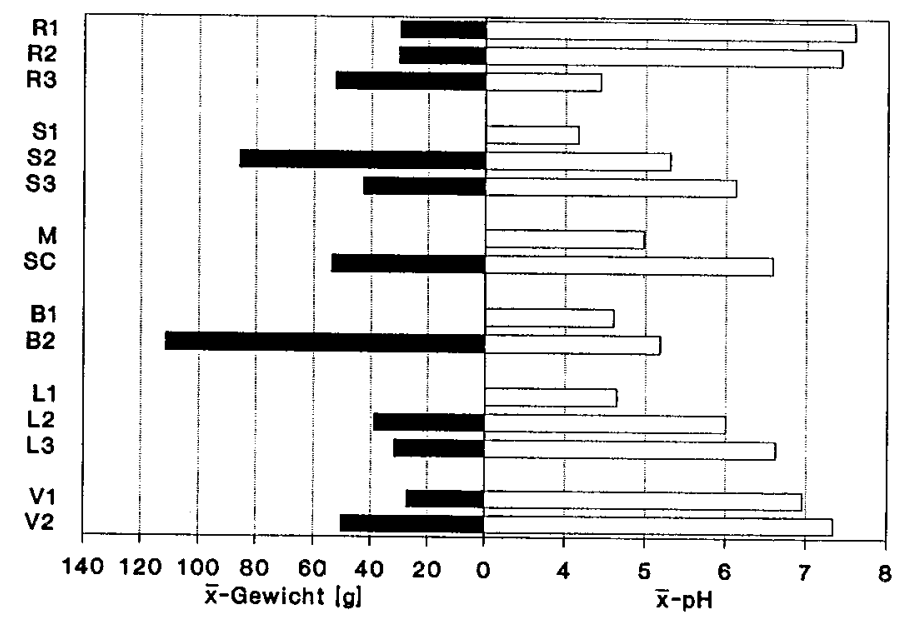

Abb. 140: Vergleich zwischen mittlerem Gewicht der Bachforellen und den mittleren $\mathrm{pH}$-Werten der Probestellen 


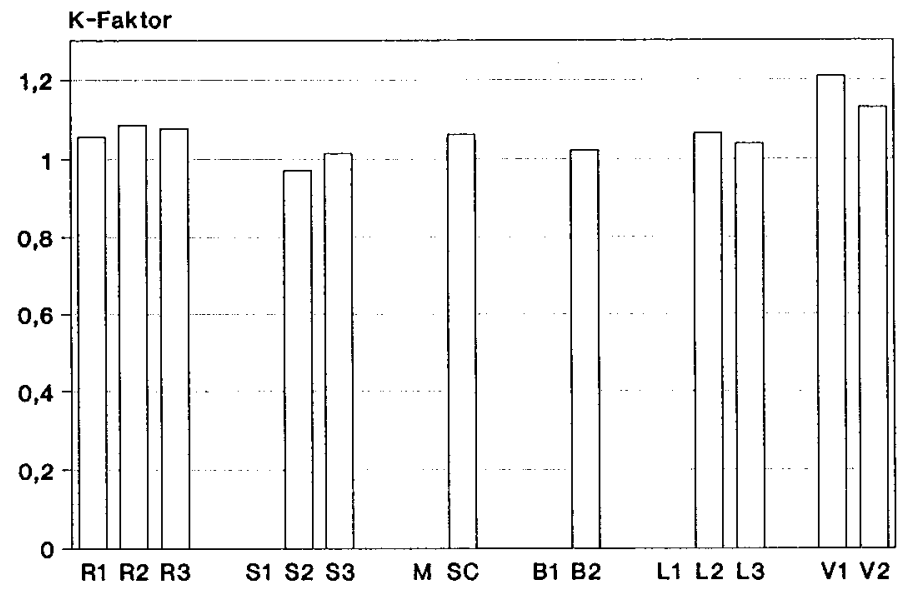

Abb. 141: Korpulenzfaktoren der Bachforellenpopulationen

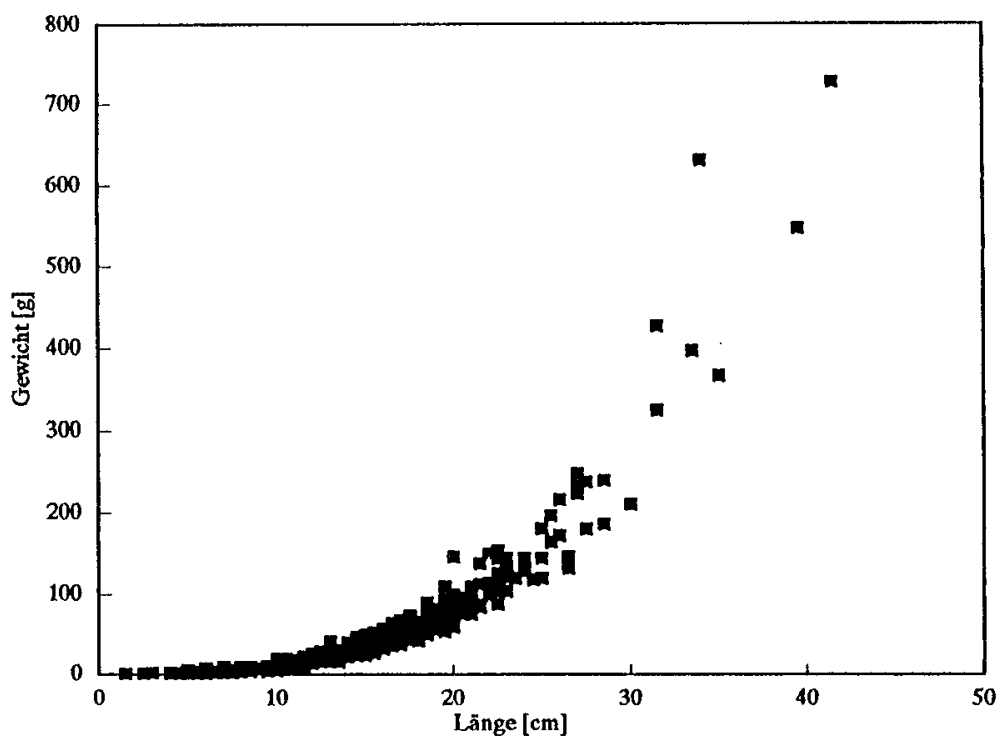

Abb. 142: Beziehung zwischen Länge und Gewicht der gefangenen Bachforellen 


\section{Diskussion}

6.1 Bedeutung der abiotischen Faktoren für die Zusammensetzung der Biozönosen

Der Harz ist aufgrund seiner exponierten geographischen Lage am Nordrand der deutschen Mittelgebirge in der Hauptwindrichtung der großen Industriegebiete an Rhein und Ruhr, der Benelux-Länder und Großbritanniens eines der am höchsten durch Luftschadstoffe belasteten Gebiete Europas. Dies wird besonders deutlich, wenn man Sulfat- und Nitratkonzentrationen aus Naß- und Trockendeposition unter Fichtenaltbeständen an ausgewählten Meßstationen der größeren deutschen Mittelgebirge im Mittel der letzten fünf Jahre miteinander vergleicht (Tab. 25). Die Schwefeldepostion liegt damit insbesondere auch weit über in Nordamerika und Skandinavien gemessenen Werten (FÜHRER et al. $1988)$.

Bei ausreichender Bodenmächtigkeit passiert der größte Teil des mit Schadstoffen angereicherten Niederschlagswassers in der Regel erst den Boden bevor er in die Gewässer gelangt. Der Boden stellt dabei einen Puffer mit Rückhalte- und Filterfunktion zwischen Atmosphäre und Hydrosphäre dar. In versauerungsempfindlichen Gebieten ist die chemische Verwitterung jedoch nur in der Lage, einen Säureeintrag auszugleichen, der $10-25 \mathrm{~kg} \mathrm{~S} / \mathrm{ha}{ }^{*} \mathrm{a}$ nicht übersteigt (SCHNOOR \& STUMM 1986). Bei den Stickoxiden werden $20 \mathrm{~kg}$ N/ha*a als ökosystemverträglicher Grenzwert versauerungsempfindlicher Gebiete angesehen (HÜSER \& REHFUESS 1988 zit. nach ZAHN 1990).

Tab. 25: Sulfat- und Nitratkonzentrationen der Gesamtdeposition unter

Fichtenaltbeständen deutscher Mittelgebirge (nach ZAHN 1990)

\begin{tabular}{lcc} 
& $\begin{array}{c}\mathrm{SO}_{4}-\mathrm{S} \\
{\left[\mathrm{kg} / \mathrm{ha}{ }^{*} \mathrm{a}\right]}\end{array}$ & $\begin{array}{c}\mathrm{NO}_{3}-\mathrm{N} \\
{\left[\mathrm{kg} / \mathrm{ha}{ }^{*} \mathrm{al}\right.}\end{array}$ \\
\hline Harz & $45,6-158,0$ & $10,8-77,5$ \\
Bayerischer Wald & $21,8-35,1$ & $7,6-14,7$ \\
Schwarzwald & $25,4-44,1$ & $7,9-26,1$ \\
Hunsrück & $32,8-39,7$ & $11,0-18,2$ \\
Kaufunger Wald & 59,0 & 19,5 \\
Rothaargebirge & 59,4 & 17,4 \\
\hline
\end{tabular}


In der Sösemulde wurden darüber hinaus in den humosen Oberböden auf allen Substraten sehr hohe Schwermetallanreicherungen festgestellt (ANDREAE \& MAYER 1989), die aus der atmosphärischen Deposition stammen müssen, da nur eine geringe geogene Schwermetallbelastung nachgewiesen werden konnte (ROOSTAI 1987, MATSCHULLAT et al. 1989). In tieferen Bodenschichten wurde auch auf dem relativ gut puffernden Untergrund des Diabas im Einzugsgebiet der nicht versauerten Alten Riefensbeek eine Versauerungsfront gefunden, oberhalb der Calcium, Magnesium, Kalium und Natrium aus dem Boden ausgewaschen sind und Aluminium freigesetzt wird (MALESSA \& ULRICH 1989). In den Einzugsgebieten der permanent versauerten Probestellen ist diese Versauerungsfront bereits bis zur wassergesättigten Zone vorgedrungen.

Eine der sichtbarsten Folgen des Schadstoffeintrags und der Bodenversauerung ist das seit Anfang der 80er Jahre großflächig voranschreitende Waldsterben, dessen Folgen für die Vegetation im Harz am deutlichsten auf dem AckerBruchberg-Zug zu sehen sind (WAGNER 1989). Die andere gravierende Folge des Schadstoffeintrags ist die Gewässerversauerung. In Gebieten mit mächtigen Böden und einem nur geringen oberflächlichen oder oberflächennahen Abfluß, wie z.B. dem größten Teil des Einzugsgebiets der Langen Bramke, sind die Gewässer erst nach erfolgter Bodenversauerung gefährdet. In den großen Gebieten des Harzes, die nur eine dünne Bodendecke aufweisen und wo insbesondere hohe Niederschläge ohne längeren Gesteins- oder Bodenkontakt in die Bäche gelangen, ist bereits seit mehreren Jahrzehnten eine Gewässerversauerung zu verzeichnen.

Indizien für einen bereits längere Zeit zurückliegenden Beginn der Versauerung sind zum Beispiel schon seit längerem nicht mehr oder nur noch eingeschränkt genutzte Fischteiche an versauerten Bächen und die Tatsache, daß in dem auf Brockengranit liegenden Oderteich bis in die 20er Jahre dieses Jahrhunderts eine Fischzucht betrieben wurde, die der Fischversorgung von St. Andreasberg durch zunächst zwei, später einer Fischerfamilie diente. Bis in den Zweiten Weltkrieg hinein wurden im See Forellen geangelt. Zu dieser Zeit wurden im Frühjahr jedoch bereits regelmäßig verendete Tiere gefunden. Später erfolgte nochmals ein erfolgreicher Besatz mit Saiblingen. Fehlgeschlagen sind dann wiederholte Besatzversuche in den 50er Jahren (MATSCHULLAT 1989). Aufgrund der sehr unruhigen Sedimentation läßt sich für den Oderteich, der heute einen mittleren $\mathrm{pH}$-Wert von 3,9 hat, kein paläolimnologischer Nachweis der Versauerungsgeschichte erbringen. 
Die Große Söse mit ihren drei Probestellen und der relativ dichten Folge von pHMessungen kann als gutes Beispiel für die Betrachtung der hydrochemischen Veränderungen durch die Versauerung dienen. Ganzjährig sehr niedrige pHWerte zeigen an $\mathrm{S} 1$ eine bereits vollständig erfolgte Versauerung der Wasser leitenden Bodenschichten im Einzugsgebiet der Probestelle an. An der ebenfalls ganzjährig stark sauren Stelle $L 1$ ist aufgrund der starken Vermoorung mit einem überwiegend oberflächennahen Fließweg in den Bach zu rechnen. In der Großen Söse lassen sich im mittleren und unteren Bereich in zeitlicher Korrelation mit einem erhöhten Abfluß periodische pH-Absenkungen beobachten, wobei die niedrigsten $\mathrm{pH}$-Werte mit $\mathrm{pH}$-Stürzen von bis zu 2,2 Einheiten nach längeren Trockenperioden in den Sommer- und Herbstmonaten erreicht werden. Die Höhe des Abflusses ist dabei nur einer von mehreren Faktoren, der das Ausmaß der pH-Erniedrigung bestimmt.

Die hohen Schadstoffkonzentrationen sind auf die in der Trockenperiode erfolgte Akkumulation von Säurebildnern in der Vegetation und den obersten Bodenschichten und deren gesammelten oberflächennahen Eintrag in den Bach zurückzuführen. Darüber hinaus ist mit einer Beteiligung der bei der Nitrifikation im Boden entstehenden Salpetersäure zu rechnen, die durch Austrocknung ebenfalls in den obersten Bodenschichten aufkonzentriert wird (ULRICH \& BÜTTNER 1985. MEESENBURG \& SCHOEN 1988). In anderen Gebieten wurde beobachtet, daß diese Starkregen-Depressionen des $\mathrm{pH}$-Werts nur eine relativ kurze Dauer von Stunden bis zu wenigen Tagen haben und mit einem starken Anstieg der Sulfat- und Metallkonzentrationen verbunden sind (BAUER et al. 1988, GÖRTZ et al. 1985, SCHOEN 1988).

Ebenfalls zu pH-Absenkungen kann es in der Großen Söse und den anderen versauerten Bächen zu Zeiten der Schneeschmelze kommen, wobei jedoch nie absolute pH-Minima gemessen werden. Eine stärkere Schneedecke war allerdings im Untersuchungszeitraum nur im Winter 1986/87 vorhanden, wohingegen sich in den beiden folgenden, milden Wintern nur zeitweilig Schneedecken aufbauen konnten, in denen nur eine geringe Schadstoffakkumulation erfolgen konnte. Säureschübe während der Schneeschmelze sind in ihrer Intensität abhängig von der Dicke der Schneedecke, der Dauer der Frostperiode, der Zahl der Zwischenschneeschmelzen und insbesondere auch von der Art der Schneeschmelze. Besonders niedrige pH-Werte können bei plötzlich einsetzendem Tauwetter verbunden mit Regen und dabei auftretendem hohen 
oberflächlichen Abfluß beobachtet werden, wobei die pH-Absenkung mit der Dicke der Schneedecke an Intensität und Dauer gewinnt (BJÄRNBORG 1983, BAUER et al. 1988). Die pH-Absenkung dauert im Vergleich mit den Starkregenereignissen relativ lange. Die Minima treten schon relativ kurz nach dem Beginn der Schneeschmelze auf, da mit den ersten $30 \%$ des Schmelzwassers bereits 50 - $80 \%$ der akkumulierten Schadstoffe in die Bäche gelangen. Danach steigt der $\mathrm{pH}$-Wert nur langsam wieder an (JOHANNESSEN \& HENRIKSEN 1978). Da bei der physiologischen Wirksamkeit die Dauer der Einwirkung eines Schadstoffs eine wesentliche Rolle spielen kann, wie auch die laborexperimentellen Untersuchungen gezeigt haben, sind die SchneeschmelzSäureschübe in ihren Auswirkungen auf die Fauna wahrscheinlich wesentlich kritischer als die Starkregen-Depressionen während des Sommerhalbjahres.

Derartige stärkere, periodische Absenkungen des $\mathrm{pH}$-Werts bei Starkregenereignissen und während der Schneeschmelze werden in allen von Gewässerversauerung betroffenen Gebieten beobachtet, wobei in der Bundesrepublik die von nur periodischen $\mathrm{pH}$-Absenkungen betroffenen Gewässerabschnitte wesentlich länger sind als die permanent stärker versauerten Strecken (FRIEDRICH \& GÖRTZ 1985, HERTER-MAIER \& SCHOEN 1988).

Die Daten der hydrochemischen Untersuchungen zeigen, daß der $\mathrm{pH}$-Wert einen Komplexfaktor darstellt und jede Änderung der Wasserstoffionenkonzentration von Änderungen anderer physiko-chemischer Faktoren begleitet wird. So ist der pH-Wert in hohem Maße mit den Konzentrationen von Aluminium, Calcium, Magnesium, DOC und Cadmium korreliert. Dadurch wächst in den versauerten Gewässern mit steigenden Protonenkonzentrationen auch die Belastung durch die ökophysiologisch wichtigen Elemente Aluminium und Cadmium, wohingegen die in entgegengesetzter Richtung wirkenden Konzentrationen an Calcium und Magnesium abnehmen. Daß sich über die untersuchten Elemente hinaus noch weitere Korrelationen ergeben, zeigen die Untersuchungen von BORG (1983), der bei Cadmium, Blei, Aluminium, Mangan, Zink, Eisen, Chrom, Kobalt und Arsen eine Korrelation zur Wasserstoffionenkonzentration feststellte.

Hydrochemisch lassen sich alle untersuchten Bäche als Silikatbäche charakterisieren, die von Karbonatbächen vor allem durch Leitfähigkeiten $<300$ $\mu \mathrm{S} / \mathrm{cm}$, niedrige Calcium- und Magnesium-Konzentrationen sowie eine geringe Gesamt- und Karbonathärte zu unterscheiden sind IOTTO \& BRAUKMANN 
1983). Eine Abwasserbelastung, die zu erhöhtem Nährstoffeintrag und sauerstoffzehrenden Prozessen führen würde, ließ sich für alle Probestellen ausschließen, so daß insbesondere eine ausreichende Sauerstoffversorgung selbst für empfindliche Arten bei Werten, die ständig im Sättigungsbereich liegen, gewährleistet ist. Von den untersuchten hydrochemischen Parametern ergeben sich nur beim Nitrat und Zink keine unmittelbaren Beziehungen zur Wasserstoffionenkonzentration. Zusammen mit den Sulfat-Konzentrationen sind die Nitrat-Belastungen in allen Bächen gleichermaßen als hoch einzustufen. Zink erreicht zwar abgesehen von der Alten Riefensbeek in allen Bächen ebenfalls relativ hohe Werte, aber nur die Meßwerte an L1 liegen deutlich über denen der anderen Stellen. Beim Blei ist neben $\mathrm{L1}$ auch an $\mathrm{S} 1$ eine erhöhte Belastung festzustellen, bleibt aber wie beim Zink noch so niedrig, daß ein Einfluß auf die Biozönose hierdurch kaum nachweisbar sein dürfte.

$\mathrm{Zu}$ den wichtigsten abiotischen Faktoren, die die Verbreitung von Fließgewässerorganismen und damit die Zusammensetzung der Biozönosen über die Gewässerchemie hinaus bestimmen, solange es zu keiner direkten anthropogenen Beeinflussungen kommt, gehören als übergeordnete Faktoren geographische Lage, Topographie, Klima, Einzugsgebietsbewaldung, Gefälle mit Breite und Tiefe und Quellentfernung des untersuchten Gewässserabschnitts (Abb. 143). Hiervon werden die gewässerinternen Faktoren Abfluß, Strömung, Substratverhältnisse, Temperatur, Licht- und Nahrungsangebot weitgehend bestimmt (BRAUKMANN 1987).

Viele dieser verbreitungsrelevanten Faktoren zeigen in den untersuchten Gewässer so geringe Unterschiede zwischen den Probestellen, daß die entscheidenden, die Zusammensetzung der Biozönose bestimmenden Faktoren relativ gut abgegrenzt werden können. Die kleineren Gewässer sind unter Berücksichtigung der hydrochemischen Untersuchungsergebnisse dem Typus des montanen Silikat-Bergbaches $\left(S M_{h}\right)$ zuzurechnen. Bei den größeren vom Acker kommenden Bächen und an B2 zeigen insbesondere die großen Abflußschwankungen, Geschiebeführung, Substratzusammensetzung und Querprofil Verhältnisse hochmontaner Silikat-Gebirgsbäche $\left({ }_{s} \mathrm{H}_{\mid}\right)$an (OTTO \& BRAUKMANN 1983, BRAUKMANN 1987). In seiner grundlegenden Arbeit konnte BRAUKMANN (1987) jedoch zeigen, daß es innerhalb der Ephemeropteren, Plecopteren und Coleopteren sowie auch anderer Tiergruppen keine Gebirgsbacharten gibt, die nicht auch in Bergbächen vorkommen. Umgekehrt gibt es auch keine Arten, die als spezifische Leit- oder 

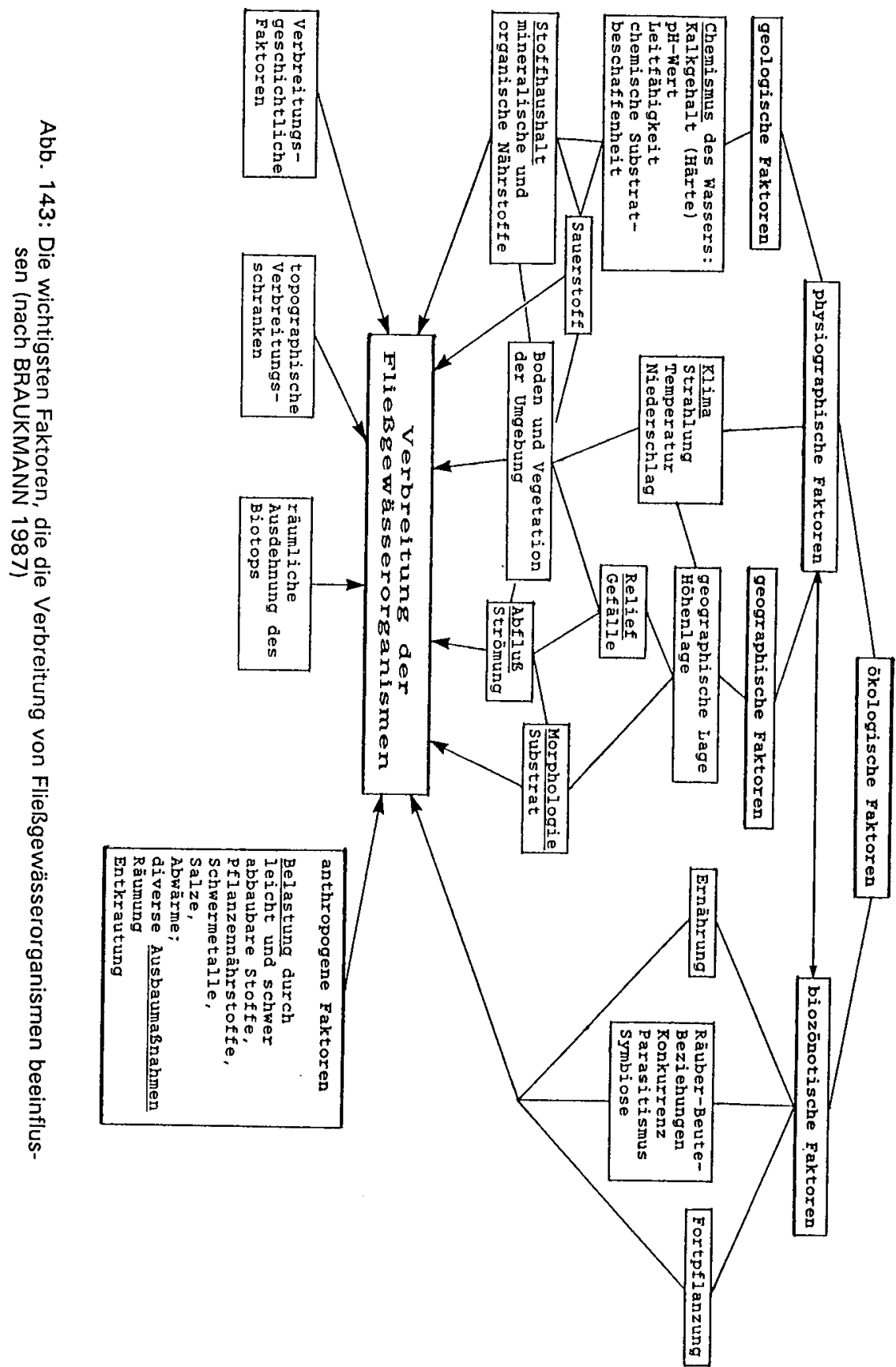
Charakterarten nur der Bergbäche angesehen werden können, was eine wichtige Voraussetzung für die durchgeführten vergleichenden Untersuchungen ist.

Neben diesen vergleichbaren physiographischen Faktoren stellt auch die Wassertemperatur einen wichtigen Faktor dar, der vor allem Einfluß hat auf den Sauerstoffgehalt und die Entwicklungsdauer der Gewässerorganismen (SCHMITZ 1957, RUTTNER 1962, HUMPESCH 1983). Den ermittelten Maximaltemperaturen und den Wetterdaten ist zu entnehmen, daß die Monatsmitteltemperaturen an allen Probestellen auch in den Sommermonaten deutlich unter $15{ }^{\circ} \mathrm{C}$ bleiben und damit auch nach den thermischen Verhältnissen eindeutig dem Rhithral zuzuordnen sind (ILLIES 1952, ILLIES 1961, ILLIES \& BOTOSANEANU 1963). Die an die Gebirgs- und Bergbäche angepaßten kaltstenothermen Arten werden daher von den Temperaturbedingungen nirgendwo beeinträchtigt.

Der Temperaturhaushalt wird insbesondere auch von der Beschattung beeinflußt, die des weiteren Einfluß auf die trophische Struktur hat. Voll beschattete Bäche zeigen eine geringe Tages- und Jahresamplitude. Die autochthone Primärproduktion ist gering. Unbeschattete Gewässer weisen dagegen größere Temperaturschwankungen und ein starkes Algenwachstum auf (MALICKY 1978, BRAUKMANN 1987). Da alle untersuchten Gewässer Waldbäche sind, an denen Fichten dominieren, die über den gesamten Gewässerverlauf betrachtet eine überwiegend volle bis teilweise Beschattung haben und an denen sich aus den Temperaturmessungen keine grundlegenden Unterschiede ergeben haben, können durch die Beschattung bedingte Unterschiede weitgehend ausgeschlossen werden. Eine erhöhte Primärproduktion ist aber dennoch an den weniger stark beschatteten Probestellen R1, S1, L1 und L2 lokal möglich.

Eine große Bedeutung kommt unter den hydromorphologischen Faktoren der Choriotopstruktur zu, die in Gebirgs- und Bergbächen vor allem von Lithal und Akal geprägt wird, wohingegen der Anteil an Psammal, Pelal und Phytal relativ gering ist (BRAUKMANN 1987). Wie durch die Cluster-Analyse deutlich gemacht werden konnte, ergeben sich hier größere Abweichungen von R1, B1 und insbesondere L1, die einen größeren Anteil feineren Materials aufweisen als die anderen Probestellen, was Auswirkungen auf die faunistische Besiedlung erwarten läßt. 
Zusammenfassend ist für die abiotischen Faktoren festzustellen, daß die Wasserstoffionenkonzentration und die mit ihr korrelierten zahlreichen hydrochemischen Parameter sowie an einigen Stellen auch die Choriotopstrukturen die abiotischen Faktoren darstellen, hinsichtlich der größere Unterschiede zwischen den Probestellen bestehen. 


\subsection{Auswirkungen der Versauerung auf die Biozönosen}

Aufgrund der abiotischen Untersuchungsergebnisse kann davon ausgegangen werden, daß in den beiden nicht versauerten Gewässern Alte Riefensbeek und Varleybach weitgehend das natürliche Spektrum der in Harzer Fließgewässern vorkommenden Arten $z \mathrm{u}$ finden ist, wenn sie keinen Belastungen durch Schadstoffe ausgesetzt sind. Soweit auch in diesen beiden Bächen gegenüber einem zu erwartenden natürlichen Hintergrund bereits höhere Konzentrationen z.B. an Sulfat und Nitrat gemessen werden, ist deren Einfluß auf die Fauna nicht genau zu bestimmen. Angesichts der ermittelten Konzentrationen und unter Berücksichtigung aller hydrochemischen Parameter ist aber bisher nicht mit signifikanten Auswirkungen zu rechnen.

Von den bei den Untersuchungen nachgewiesenen typischen Gebirgs- sowie Gebirgs- und Bergbacharten nach BRAUKMANN (1987) (Tab. 26) kommen alle in zumindest einem der beiden nicht versauerten Bäche vor, wobei keine Art, die ausschließlich in einem der nicht versauerten Gewässer auftritt, in einem der versauerten Bäche gefunden werden kann. Acht der Ephemeropteren- und alle neun Plecopteren- und drei Coleopteren-Arten sind in beiden Gewässern anzutreffen. Während alle Taxa der Plecopteren und Coleopteren auch in schwach versauerten Bächen gefunden werden können, leben dort weder Ancylus fluviatilis noch Gammarus fossarum, und von den typischen

Tab. 26: Typische Gebirgsbacharten bzw. Gebirgs- und Bergbacharten nach BRAUKMANN (1987), die in den Untersuchungsgewässern vorkommen

\begin{tabular}{|c|c|}
\hline Mollusca & Ancylus fluviatilis \\
\hline Amphipoda & Gammarus fossarum \\
\hline Ephemeroptera & $\begin{array}{l}\text { Baetis alpinus, Baetis melanonyx, Epeorus sylvicola; } \\
\text { Rhithrogena semicolorata, Ecdyonurus spp., Ephemerella } \\
\text { mucronata, Habroleptoides modesta, Baetis muticus, } \\
\text { Baetis lutheri, Habrophlebia lauta }\end{array}$ \\
\hline Plecoptera & $\begin{array}{l}\text { Chloroperia tripunctata; Protonemura spp., Siphonoperla } \\
\text { torrentium, Leuctra spp., Isoperla spp., Brachyptera } \\
\text { seticornis, Perlodes microcephalus, Amphinemura spp., } \\
\text { Nemoura spp. }\end{array}$ \\
\hline oleo & lus angustatus, Limnius perrisi, Oreodytes rivalis \\
\hline
\end{tabular}


Ephemeropteren-Arten fehlen Ephemerella mucronata und Baetis lutheri. Diese faunistischen Ergebnisse unterstreichen die Berechtigung der Festlegung von Alter Riefensbeek und Varleybach als Referenzgewässer für die vergleichenden Untersuchungen.

Die mit der Gewässerversauerung verbundene tiefgreifende Änderung der Struktur der Biozönosen ist zum einen auf direkte Schädigungen der Organismen durch die hohen Schadstoffkonzentrationen zum anderen auf daraus resultierende indirekte Auswirkungen zurückzuführen, durch die es zu Veränderungen in den Räuber-Beute-Beziehungen und in der Nahrungskette kommt.

Weltweit konnte bisher bei alten Untersuchungen zu den Auswirkungen der Versauerung auf Gewässerbiozönosen ein drastischer Rückgang der Gesamtartenzahlen, der mehr oder weniger alle Tiergruppen betrifft, mit zunehmenden Wasserstoffionenkonzentrationen festgestellt werden (vgl. LENHART \& STEINBERG 1984, DECKER 1987). Mollusken, Crustaceen, Ephemeropteren und Fische können dabei, wie auch im Harz zu beobachten, aus versauerten Gewässern vollständig verschwinden. Darüber hinaus werden sowohl Abnahmen der Besiedlungsdichten (HEITKAMP et al. 1985, BAUER et al. 1988) als auch keine wesentlichen Unterschiede in der Gesamtabundanz zu nicht versauerten Fließgewässern (HARRIMAN \& MORRISON 1982, TOWNSEND et al. 1983, SIMPSON et al. 1985, WEATHERLEY et al. 1989) beschrieben.

Aufgrund der maximal über drei Jahre durchgeführten Untersuchungen im Harz zeichnet sich $a b$, daß die Benthos-Besiedlungsdichten versauerter Gewässer meistens unter denen vergleichbarer nicht versauerter Probestellen liegen. Relativ konstante pH-Verhältnisse im sauren Bereich scheinen eher zu höheren Abundanzen und damit zu einer Verringerung der Unterschiede zu führen als stärkere nur periodische Absenkungen des $\mathrm{pH}$-Werts. An den permanent stark versauerten Gewässern kann es verstärkt zu Massenentwicklungen einzelner Arten kommen, die die Abundanz nicht versauerter Gewässer weit übersteigt, wie an Probestelle S1 beobachtet werden konnte. Bei der Emergenzdichte erreichen die versauerten Stellen alle die Werte der nicht versauerten Gewässer, übertreffen diese mit zunehmender Versauerung meist sogar sehr deutlich, was vor allem auf den steigenden Anteil der Plecopteren an der Gesamtbesiedlung zurückgeführt werden kann. Die nicht in der Emergenz erfaßten Coleopteren und Gammariden nehmen hingegen stark ab bzw. kommen in versauerten Gewässern nicht mehr vor. Allgemein gilt für Artenzahlen und Besiedlungsdichten das 
zweite biozönotische Grundprinzip, wonach einseitige und extreme Lebensbedingungen zu Artenarmut führen und die vorhandenen Arten dafür einen größeren Individuenreichtum zeigen (THIENEMANN zit. nach SCHAEFER \& TISCHLER 1983), wovon vor allem einige Plecopteren-Arten profitieren.

Die Ursachen für die Toxizität versauerter Gewässer liegen primär in den erhöhten Wasserstoffionenkonzentrationen und dem damit verbundenen Anstieg der Konzentrationen an monomeren anorganischen Aluminium-Species und Schwermetallen (vgl Kap. 5.3). Durch experimentelle Untersuchungen wurden teilweise artspezifisch sehr unterschiedliche Beteiligungen der einzelnen Belastungsfaktoren an der Toxizität festgestellt.

Am intensivsten sind bisher Fische untersucht worden. Negative Auswirkungen der Wasserstoffionenkonzentrationen sind bei der Bachforelle bei pH-Werten < 5,0 festzustellen (LEIVESTAD et al. 1980). In Verbindung mit erhöhten Aluminium-Konzentrationen kommt es jedoch bereits im pH-Bereich zwischen 5 und $6 \mathrm{zu}$ einem Anstieg der Mortalitätsraten, wobei die monomeren anorganischen Aluminium-Species die höchste toxische Wirkung zwischen pH 5,1 und 5,5 erreichen (NEVILLE \& CAMPBELL 1988). Bei pH 4,5 und $\geq 0,2 \mathrm{mg} / \mid \mathrm{Al}{ }^{3+}$ steigt die Mortalitätsrate auf $100 \%$ (BAKER \& SCHOFIELD 1982). Fischbrut reagiert noch wesentlich empfindlicher. Bei Dottersackbrut wurden subletale Schädigungen in Form von Blutungen bereits bei $\mathrm{pH}$-Werten zwischen 5,5 und 6,8 und Aluminium-Konzentrationen von $0,07 \mathrm{mg} / \mathrm{l}$ festgestellt (LUBIENIECKI \& STEINBERG 1987). Als pathologische Befunde sind an den Fischen vor allem Schädigungen an den Kiemen zu beobachten (MUNIZ \& LEIVESTAD 1980 , FISCHER-SCHERL et al. 1988), die das zentrale Organ für den Gasaustausch, die Säure-Basen-Regulation und die Steuerung des osmotischen Gleichgewichts darstellen (RANDALL et al. 1982, EDDY 1982). Die Folgen davon sind Veränderungen an den Nieren und der Elektrolytgehalte des Blutplasmas (FISCHERSCHERL et al. 1988).

Während bei den Fischen eine Beteiligung des Aluminiums an der Toxizität bei allen untersuchten Arten nachgewiesen werden konnte, ergeben sich bei den Invertebraten nicht so einheitliche Ergebnisse der experimentellen Untersuchungen. BAUER et al. (1988) sehen anhand der Ergebnisse von ORMEROD et al. (1987), HALL et al. (1987), ALLARD \& MOREAU (1987), HAVAS \& HUTCHINSON (1982) und HAVAS \& LIKENS (1985) einen Trend, der andeutet, daß Aluminium bei Konzentrationen $<1 \mathrm{mg} / \mathrm{l}$ und bei $\mathrm{pH}$-Werten $<5,0$ keine 
zusätzliche Mortalität bedingt. Aber auch unter diesen Bedingungen ist als subletaler Effekt eine erhöhte organismische Drift festzustellen. PH-Werte $>5,0$ und Aluminium-Konzentrationen $>1,0 \mathrm{mg} / \mathrm{l}$ können dagegen zu einer Erhöhung der Mortalitätsraten führen. Dies würde eine Übereinstimmung, auf allerdings höherem Konzentrationsniveau, mit den Ergebnissen bei den Fischen bedeuten. Die in Ergänzung zu den Freilandarbeiten im Harz durchgeführten laborexperimentellen Untersuchungen haben im Gegensatz zu dem von BAUER et al. (1988) festgestellten Trend bei Epeorus sylvicola, Ecdyonurus venosus und insbesondere bei Habroleptoides modesta auch bei niedrigeren $\mathrm{pH}-$ Werten bei Aluminium-Konzentrationen von etwa $1 \mathrm{mg} / \mathrm{l}$, wie sie auch im Freiland gemessen werden, zu einer Erhöhung der Mortalitätsraten geführt (AVERMANN 1990a). Dagegen konnte für eine Reihe weiterer Arten ein Einfluß des Aluminiums bei $\mathrm{pH}-$ Werten $<5,0$ ebenfalls nicht festgestellt werden.

Oft bereits frühzeitig auftretende Effekte der Gewässerversauerung bei den Invertebraten sind die ansteigenden Driftraten versauerungssensibler Arten (RADDUM \& FJELLHEIM 1987, WEATHERLEY et al. 1988, WASSMANN 1987, MERRETT et al. 1991) und Änderungen der Respirationsraten, wie sie bei Ephemeropteren gemessen wurden (HERRMANN \& ANDERSSON 1986). Als direkte physiologische Schädigungen konnten beispielsweise bei Crustaceen Störungen der Osmoregulation und Eientwicklung (HAVAS et al. 1984), bei Gammarus fossarum Fehlregulationen im lonenhaushalt (MEINEL \& MATTHIAS 1982) oder bei Ephemeropteren Aluminium-Präzipitationen auf den Kiemen mit Störungen der Osmoregulation und des lonentransports (HERRMANN \& ANDERSSON 1986) festgestellt werden.

Bei den Schwermetallen wurden zwar kaum Überschreitungen der Trinkwassergrenzwerte festgestellt, gerade Schwermetallgehalte können sich aber in ihrer Wirkung auf den Organismus potenzieren. Die Analysen haben an den meisten Probestellen deutliche Überschreitungen der natürlichen Konzentrationsbereiche ergeben. Besondere Gefahren gehen hierbei von Blei, Zink und Cadmium aus, die zusammen eine synergistische Wirkung im Vergleich mit den einzelnen Metallen aufweisen (REICHENBACH-KLINKE 1978). Die Toxizitätswirkung von Metallen nimmt in der Regel mit höheren $\mathrm{pH}$ - und Calcium-Werten und niedrigerer Temperatur ab (SCHWEIGER 1957, PHILLIPS 1980). Dies gilt auch für das Aluminium, bei dem besonders Huminstoffe durch Komplexbildung für eine Verringerung des Anteils toxischer Al-Verbindungen sorgen können (McCAHON \& PASCOE 1989). 
Wie schon die autökologischen Betrachtungen gezeigt haben, ergibt sich häufig eine große Diskrepanz zwischen den experimentell ermittelten Toleranzwerten und den Daten, die durch Freilandbeobachtungen erhoben wurden und in der Regel eine wesentlich geringere Toleranz erwarten lassen. Die Ursachen hierfür sind sehr vielfältig. Laborexperimentelle Untersuchungen basieren meist nur auf Toxizitätstests an wenigen, gut zu hälternden Organismen, die von einheitlichem Alter und einheitlicher Größe sind, so daß keine heterogene Antwort, wie sie die natürliche Population liefern würde, erwartet werden kann (CAIRNS 1981). Die sensibelsten Phasen stellen bei Insekten die Häutungen und die Emergenz dar (BELL 1971). Nahrung, physiologische Wechselwirkungen, Substrat, Strömung und Lichtverhältnisse sind nur einige Faktoren, die im Experiment von den natürlichen Bedingungen fast immer abweichen (BUIKEMA \& BENFIELD 1979, JERNELON 1978). Darüber hinaus fehlen die Wechselwirkungen zwischen den Arten, die Konkurrenz und der Räuberdruck. Experimentelle Untersuchungen sind daher zwar notwendig, um im Zusammenhang mit der Gewässerversauerung die Auswirkungen auf einzelne Arten zu beschreiben, sie können aber allein keine zufriedenstellenden Ergebnisse liefern, um die Veränderungen im aquatischen Ökosystem zu analysieren, zu denen auch die indirekten Auswirkungen der Gewässerversauerung gehören.

Unter den indirekten Auswirkungen der Versauerung ist die Veränderung der trophischen Strukturen ein zentraler Punkt. In versauerten Fließgewässern ist nicht zwangsläufig mit einer verminderten Primärproduktion zu rechnen (PLANAS \& MOREAU 1986, STOKES 1986), wofür auch die relativ geringen Unterschiede bei den Besiedlungsdichten sprechen. Eine Verringerung der bakteriellen Abbautätigkeit konnte wiederholt nachgewiesen werden (BAKER et al. 1983, HILDREW et al. 1984, BURTON et al. 1985, PALUMBO et al. 1987, GARDEN \& DAVIES 1989), was zu einer Anreicherung von organischer Substanz in den Gewässern führen kann (BRETTFELD 1987, BURTON \& ALLAN 1986, KIMMEL et al. 1985). Durch den geringeren mikrobiellen Aufschluß weist der Detritus zudem einen geringeren Nährstoffgehalt auf (HAMIER 1987).

Wie RÜDDENKLAU (1989) für die im Harz untersuchten Bäche anhand der Trichopteren zeigen konnte, bleibt dies nicht ohne Auswirkungen auf die Biozönosen im Hinblick auf die Anteile der funktionellen Ernährungstypen an der Gesamtabundanz. Während in den nicht versauerten Bächen Weidegänger mit die höchsten Abundanzen erreichen, wird die Trichopteren-Zönose der versauerten Bäche vor allem von Räubern und Zerkleinerern bestimmt. In Bezug auf die 
Gesamtzönose ergeben sich ebenfalls deutliche Veränderungen. Durch den drastischen Rückgang der Ephemeropteren und phytophagen Coleopteren an den versauerten Probestellen sinkt der Anteil der Weidegänger sehr stark, während gleichzeitig die Dominanz der saprophagen Filipalpia-Arten der Plecopteren ansteigt und damit der Anteil der Zerkleinerer stark zunimmt. Die Anteile der räuberischen Plecopteren- und Coleopteren-Arten bewegen sich an allen Stellen im normalen Schwankungsbereich von unter 1 bis maximal $3 \%$ der Abundanz. Die Zahl der räuberischen Arten nimmt aber mit zunehmender Versauerung stark ab, wovon bei den Plecopteren Diura bicaudata, bei den Coleopteren Agabus guttatus und bei den Trichopteren Plectrocnemia conspersa profitieren, die an den stark versauerten Stellen ihre höchsten Abundanzen erreichen. Eine detaillierte Auswertung der Veränderungen im Trophiegefüge der Probestellen für die Gesamtzoozönose erscheint erst nach Abschluß der Bearbeitung aller Tiergruppen sinnvoll .

Auf die Abnahme der Abundanzen bei Weidegängern und Filtrierern sowie die Zunahme der Dichte der Zerkleinerer mit abnehmenden $\mathrm{pH}$-Werten weisen auch die Ergebnisse einer Reihe anderer Untersuchungen hin (EGGLISHAW 1964, 1968, FRIBERG et al. 1980, TOWNSEND et al. 1983, OTTO \& SVENSSON 1983, HILDREW et al. 1984, MACKAY \& KERSEY 1985). Diskutiert wird eine selektive Förderung der Zerkleinerer aufgrund eines größeren und gleichmäßigeren Nahrungsangebots über das Jahr durch den langsameren mikrobiellen Abbau. Positiv für die säuretoleranten Arten unter den Plecopteren ist insbesondere das Entfallen der Konkurrenz durch die Gammariden, die in nicht versauerten Gewässern einen großen Teil der Zerkleinerer stellen können. Ursachen für den starken Rückgang der Weidegänger können zum einen in Veränderungen der submersen Vegetation, insbesondere der epilithischen Algen begründet sein (WINTERBOURN et al. 1985, ORMEROD et al. 1987a), zum anderen können unter den Weidegängern aber auch ausreichend säuretolerante Arten fehlen. 
6.3 Möglichkeiten und Wege zu einer biologischen Indikation der Gewässerversauerung

Ein Ziel der Untersuchungen war es, Möglichkeiten und Wege einer biologischen Indikation der Gewässerversauerung zu prüfen. An permanent stark versauerten Probestellen wie S1 und L1 besteht für eine biologische Indikation scheinbar keine Notwendigkeit, da hier der Versauerungszustand des Gewässers bereits durch wenige $\mathrm{pH}-$ Messungen zu ermitteln ist. Betrachtet man jedoch den Gang des $\mathrm{pH}$-Wertes an den anderen versauerten Probestellen mit ihren starken Schwankungen zwischen dem neutralen und sauren Bereich, so wird schnell deutlich, wie schwierig es sein kann, den Versauerungsgrad anhand einmaliger oder weniger $\mathrm{pH}-$ Messungen richtig einzuschätzen. Diese starken $\mathrm{pH}$-Schwankungen unterliegenden Bereiche stellen aber den Großteil der von der Versauerung betroffenen Gewässerabschnitte. Wie die Untersuchungsergebnisse gezeigt haben, manifestieren sich die verschiedenen Grade der Versauerung über einen längeren Zeitraum in den faunistischen und biozönotischen Strukturen, was für eine Bioindikation genutzt werden kann.

Wiederholt ist die Verwendung des Artenfehlbetrags nach KOTHE (1962) als ein Weg der biologischen Versauerungsindikation vorgeschlagen worden (MATTHIAS 1983, UBA 1987). Bei der praktischen Anwendung tritt jedoch das Problem der Festlegung einer verläßlichen Bezugsartenzahl auf, da die Bäche von der Quellregion her versauern und damit die Möglichkeit nicht gegeben ist, in ein und demselben Gewässer ober- und unterhalb der Schadstoffbelastung die Artenzahlen miteinander zu vergleichen. Es wird ein Referenzgewässer benötigt, bei dem vorher sichergestellt werden muß, daß es sich aufgrund der Übereinstimmung der sich durch die Versauerung nicht verändernden Faktoren für einen Vergleich eignet, wozu bereits relativ umfangreiche Untersuchungen notwendig sein können. In vielen betroffenen Gebieten stehen zudem keine unversauerten Vergleichsgewässer mehr zur Verfügung.

Für die Berechnung des Artenfehlbetrags der untersuchten Harzbäche kann aufgrund der Ergebnisse der abiotischen Untersuchungen die Alte Riefensbeek als Referenzgewässer herangezogen werden (Abb. 144). Die sich ergebenden Artenfehlbeträge ermöglichen zwar grundsätzlich eine korrekte identifizierung der stärker versauerten Probestellen, es sind jedoch auch sehr leicht Fehlinterpretationen möglich. So zeigen zum Beispiel alle Probestellen der Langen Bramke einen größeren relativen Artenfehlbetrag als es ihrem tatsächlichen Versaue- 


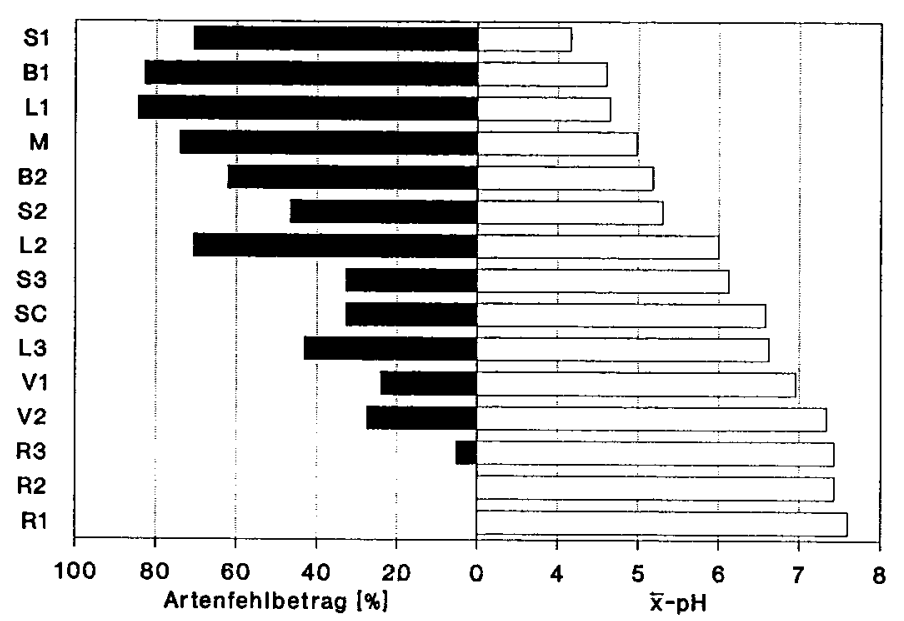

Abb. 144: Artenfehlbetrag in Abhängigkeit von den mittleren $\mathrm{pH}-$ Werten

rungsgrad entspricht, und der nicht versauerte Varleybach rückt sehr dicht an die Probestellen mit episodischen Säureschüben heran. Läßt sich anhand der Werte schon keine Grenze zwischen nicht versauerten und versauerten Gewässern ziehen, so scheint eine Unterteilung in verschiedene Versauerungsgrade völlig ausgeschlossen.

Ein weiterer Ansatz zur Beurteilung der Versauerung nutzt vor allem die autökologischen Ergebnisse zur Säuretoleranz charakteristischer stenoioner Arten (ENGBLOM \& LINGDELL 1984, RADDUM \& FJELLHEIM 1984, UBA 1987). In Frage kommen hierfür in erster Linie die als besonders säuresensibel geltenden Gammariden, Ancylus fluviatilis sowie verschiedene Ephemeropteren. Für viele dieser Arten sind bis heute die verbreitungsregulierenden Faktoren jedoch nicht ausreichend bekannt. So lassen sich trotz vielfältiger Ansätze die Verbreitung von Gammarus fossarum und Gammarus pulex im Mittelgebirgsraum nicht immer zufriedenstellend erklären (WUNDSCH 1922, DITTMAR 1955, SCHRIMPF \& FOECKLER 1985). In einem Gewässer können Gammariden wie auch Ancylus fluviatilis fehlen, ohne daß dieses versauert ist. Wie die autökologischen 
Betrachtungen außerdem gezeigt haben, wurden bei Untersuchungen teilweise sehr unterschiedliche Versauerungstoleranzen für einzelne Arten ermittelt, die sehr stark von den sonstigen physiko-chemischen Bedingungen abhängig zu sein scheinen. Laborexperimentell ermittelte Werte lassen sich ebenfalls nur unter großen Vorbehalten auf das Freiland übertragen. Die Überprüfung des Vorkommens bestimmter versauerungssensitiver Arten und die Berechnung des Artenfehlbetrags erlauben daher nur sehr begrenzte Aussagen über den Versauerungszustand eines Fließgewässers.

Das klassische System zur biologischen Bestimmung der Gewässergüte bei organischen, fäulnisfähigen Verunreinigungen ist das Saprobiensystem (KOLKWITZ \& MARSSON 1902, KOLKWITZ \& MARSSON 1908, KOLKWITZ \& MARSSON 1909). Für die Beurteilung werden hierbei nicht nur bestimmte Arten als Leitorganismen herangezogen, sondern es wird die biozönotische Gesamtstruktur berücksichtigt, da neben qualitativen Merkmalen auch der quantitativen Besiedlung eine große Bedeutung zukommt. Die Ergebnisse der Untersuchungen an 104 versauerten und nicht versauerten Fließgewässerprobestellen in Wales ermöglichte unter Heranziehung halbquantitativer faunistischer Daten eine Unterscheidung von vier Probestellengruppen, die sich hinsichtlich der physikochemischen Faktoren am stärksten in ihren $\mathrm{pH}$-Werten und Aluminium-Konzentrationen unterscheiden. Es konnte ein Schlüssel mit Indikatororganismen erstellt werden, anhand dessen eine relativ korrekte Zuordnung jeder der 104 Probestellen zu einer der vier Gewässergruppen möglich ist (WADE et al. 1989).

Zur Erstellung eines solchen "Versauerungsbestimmungsschlüssels" für den Harz ist die Datengrundlage der vorliegenden Untersuchungen zu klein. Erforderlich ist hierbei auch die Einbeziehung möglichst aller Makrobenthosgruppen und die Berücksichtigung von Arten, die nur niedrige Abundanzen haben. Die Betrachtung der Dominanzstrukturen der Harzuntersuchungen hat gezeigt, daß sich hierin gravierende Unterschiede zwischen den Probestellen ergeben, wobei zu prüfen ist, ob diese allein unter Berücksichtigung weitestgehend nur der dominanten Arten sowie einiger weiterer leicht erfaßbarer Kriterien ausreichen, um eine Versauerungsindikation für die untersuchten Bäche zu ermöglichen.

Das am stärksten differenzierte Datenmaterial steht über die Emergenzerfassungen zur Verfügung. Eine Zusammenstellung der Probestellen in der Reihenfolge ihrer mittleren $\mathrm{pH}$-Werte mit Auflistung der Arten, die eine Dominanz $\geq 5 \%$ erreichen, zeigt, daß sich für die von ihrem $\mathrm{pH}$-Gang her als nicht, schwach, 
mäßig und stark versauert definierten Bereiche relativ charakteristische Artenund Dominanzstrukturen ergeben (Tab. 27).

Die nicht versauerten Probestellen weisen alle eine hohe Dominanz von Leuctra aurita auf. $\mathrm{Zu}$ den dominanten Taxa gehören immer die Gattung Baetis oder eine andere Ephemeroptere, an vier der fünf Stellen Leuctra inermis und an drei Stellen Amphinemura sulcicollis. Keine dieser Arten ist unter den dominanten Arten der stark versauerten Stellen zu finden. Die drei am stärksten versauerten Probestellen, ohne L1, zeichnen sich hingegen dadurch aus, daß Leuctra rauscheri die Art mit der höchsten Dominanz ist. Gemeinsam mit ihr kommen Protonemura auberti und Amphinemura standfussi vor. Weitere dominante Arten sind Leuctra pseudocingulata, Nemoura cambrica, Leuctra nigra und Nemurella pictetii, die ebenfalis an keiner der nicht versauerten Stellen mit höheren Abundanzen gefunden werden können. Die an $\mathrm{L} 1$ dominierenden Arten Nemurella pictetii und Leuctra nigra sind auch an den anderen stark versauerten Stellen vertreten, erreichen dort aber nur geringe Dichten.

Die mäßig sauren Probestellen B2, S2 und L2 sind ebenfalls durchgehend durch die an den stark sauren Probestellen dominierenden Arten charakterisiert. Daneben treten an B2 und S2 mit Brachyptera seticornis, Leuctra pseudosignifera und Siphonoperla torrentium auch einzelne Arten unter den dominanten Arten auf, die weder für die stark sauren noch für die nicht versauerten Probe-

Tab. 27: Die eudominanten und dominanten Taxa der Emergenzerfassung

\section{1. "Stark versauert"}

s1

Leuctra rauscheri

Protonemura auberti Amphinemura standfussi Leuctra pseudocingulata

M

Leuctra rauscheri

Protonemura auberti

Amphinemura standfussi

Leuctra pseudocingulata
B1

Leuctra rauscheri Amphinemura standfussi Nemoura cambrica
L1

Nemurella pictetii

Leuctra nigra 
Tab. 27 (Forts.)

2. "Mäßig versauert"

\section{B2}

Protonemura auberti

Leuctra rauscheri

Amphinemura standfussi

Leuctra pseudocingulata

Brachyptera seticornis

Nemoura cambrica s2

Leuctra rauscheri

Leuctra pseudocingulata

Amphinemura standfussi

Leuctra pseudosignifera

Nemoura cambrica

Protonemura auberti

Baetis spp.

Siphonoperla torrentium

\section{3. "Schwach versauert"}

\section{\$3}

Amphinemura standfussi

Baetis spp.

Leuctra inermis

Siphonoperla torrentium

Protonemura auberti

Leuctra pseudocingulata

Leuctra aurita

Leuctra rauscheri

\section{4. "Nicht versauert"}

\section{V1}

Leuctra aurita

Leuctra inermis

Protonemura intricata

Baetis spp.

Amphinemura sulcicollis

Protonemura auberti

\section{R2}

Leuctra aurita

Leuctra inermis

Baetis spp.

Leuctra albida
SC

Leuctra aurita

Baetis spp.

Chloroperla tripunctata

Leuctra inermis

\section{V2}

Leuctra aurita

Leuctra albida

Habrophlebia lauta

\section{R1}

Leuctra inermis

Amphinemura sulcicollis

Protonemura nitida

Leuctra aurita

Baetis spp.

Protonemura auberti

Leuctra braueri

\section{L2}

Amphinemura standfussi Leuctra pseudocingulata Nemurella pictetii

\section{L3}

Leuctra nigra

Leuctra aurita

Protonemura intricata

Amphinemura standfussi

Nemoura cambrica

Protonemura auberti

Leuctra pseudocingulata

\section{R3}

Leuctra inermis

Leuctra aurita

Baetis spp.

Protonemura intricata

Leuctra albida 
stellen typisch sind. Die Zuordnung von L2 zu den mäßig versauerten Stellen erhält durch das Emergenzspektrum eine Rechtfertigung erst bei Berücksichtigung auch der nur in geringer Zahl vorkommenden Arten, unter denen Baetis und Protonemura meyeri sind, die an stark versauerten Stellen fehlen.

An den schwach versauerten Probestellen S3, SC und L3 finden sich dagegen stets sowohl für den nicht versauerten als auch für den stark versauerten Bereich charakteristische Arten in unterschiedlicher Zahl. Danach tendieren S3 mit Amphinemura standfussi als Art mit der höchsten Dominanz und der ebenfalls vertretenen Protonemura auberti sowie $L 3$ mit drei für die Versauerung typischen Arten mehr zu den stärker sauren Probestellen, wohingegen SC insbesondere durch Leuctra aurita und den ebenfalls für den nicht versauerten Bereich charakteristischen Leuctra inermis und Baetis mehr zu den nicht versauerten Stellen zu zählen ist, was bereits durch den biozönotischen Ähnlichkeitsvergleich zum Ausdruck kam (vgl. Kap. 5.5.5). Die größten Abweichungen und damit verbunden größere Unsicherheiten bei der Zuordnung zu einer der Versauerungsgruppen zeigen die Probestellen der Langen Bramke. Dies dürfte mit darauf zurückzuführen sein, daß die Lange Bramke der kleinste der untersuchten Bäche ist.

Die meisten der charakteristischen Arten aller vier Versauerungsgruppen sind sehr säuretolerant. Auch in den nicht versauerten Bächen sind unter den dominanten Arten nur wenige zu finden, die in versauerten Gewässern nicht vorkommen. Die starke Abnahme der Artenzahlen mit zunehmender Versauerung läßt sich für die Unterscheidung des Versauerungsgrades nur ergänzend nutzen, da zwischen den Gruppen größere Überschneidungen auftreten.

Daß es in Abhängigkeit vom Ausmaß der Versauerung zu relativ charakteristischen Dominanzstrukturen nicht nur in den untersuchten Bächen kommt, zeigen Vergleiche mit den Ergebnissen von Emergenzuntersuchungen in anderen Gewässern. Die Arten mit den höchsten Abundanzen im mit $\mathrm{pH}$-Werten $<4$ stark versauerten Bereich der Oder im Harz sind Leuctra rauscheri, Leuctra nigra, Amphinemura standfussi, Protonemura auberti und Leuctra pseudocingulata (GRASHOF 1972) und damit ausschließlich Arten, die auch bei den eigenen Untersuchungen die stark versauerten Bereiche charakterisieren. 
Ein Vergleich mit der von MATTHIAS (1983) im benachbarten Kaufunger Wald ermittelten Emergenzstruktur ergibt relativ große Gemeinsamkeiten, vor allem auch unter Berücksichtigung der dortigen geringeren Gewässergröße. Die sechs Arten mit den höchsten Abundanzen des nicht versauerten Wengebachs (Protonemura intricata, Baetis spp., Leuctra inermis, Protonemura nitida, Amphinemura sulcicollis und Leuctra albida) sind alle auch unter den dominanten Arten der nicht versauerten Harzbäche zu finden. Abgesehen von Amphinemura sulcicollis zeichnen sich die drei stark versauerten Gewässer im Kaufunger Wald mit Protonemura auberti, Nemurella pictetii, Nemoura cinerea und Leuctra nigra durch auch im Harz typische Vertreter der stark versauerten Bereiche aus. Im Endschlagbach, der einen mittleren $\mathrm{pH}-$ Wert von 4,7 hat, tritt darüber hinaus Siphonoperla torrentium auf, wodurch die etwas schwächere Versauerung bereits in der Emergenzstruktur zum Ausdruck kommt. Die Eingruppierung der Nieste im Bereich zwischen stark und nicht versauert ergibt sich durch mehrere Arten der stark versauerten Bereiche bei gleichzeitiger Anwesenheit typischer Arten des nicht versauerten Bereichs. Amphinemura sulcicollis ist im Kaufunger Wald eine Art, die in allen Bächen in größerer Zahl vorkommt, so daß der im Harz scheinbar pH-abhängige Dominanzwechsel zwischen Amphinemura sulcicollis und Amphinemura standfussi wohl eher auf andere Faktoren zurückzuführen ist.

Der Vergleich mit Emergenzuntersuchungen an wesentlich kleineren Fließgewässern des Hils (WESTERMANN 1990) zeigt ebenfalls, daß selbst hier noch eine relativ gute Versauerungsklassifizierung anhand der Ergebnisse der Harzuntersuchungen möglich ist. Die Unterschiede sind jedoch größer als im Vergleich mit den Bächen des Kaufunger Waldes.

Eine entsprechende Darstellung der Daten der Benthosaufsammlungen ergibt ebenfalls überwiegend unterschiedliche Dominanzstrukturen zwischen den verschiedenen pH-Gruppen (Tab. 28). Während bei den Emergenzdaten die zusätzliche Berücksichtigung der Artenzahlen nur einen begrenzten Beitrag zur Klassifizierung leisten kann, sollten sie bei der Betrachtung der Benthosdaten mit herangezogen werden (vgl. Abb. 66).

An allen stark versauerten Probestellen gehören Leuctra und Nemoura zu den dominanten Plecopteren-Gattungen. Des weiteren können noch Protonemura und Amphinemura auftreten. Ephemeropteren fehlen in dieser Gruppe wie auch an den mäßig versauerten Stellen völlig, an denen die dominanten Arten von 
Tab. 28: Die eudominanten und dominanten Taxa der gemeinsamen Benthosbeprobungen

\section{1. "Stark versauert"}

S1

Leuctra spp.

Nemoura spp.

Protonemura spp.

\section{M}

Leuctra spp.

Protonemura spp.

Nemoura spp.

\section{2. "Mäßig versauert"}

B2

Leuctra spp.

Amphinemura sp.

Nemoura spp.

Protonemura spp.

Brachyptera seticornis

\section{3. "Schwach versauert"}

\section{S3}

Leuctra spp.

Baetis spp.

Brachyptera seticornis

\section{4. "Nicht versauert"}

V1

Leuctra spp.

Baetis spp.

Amphinemura sp.

Esolus angustatus

Limnius perrisi
B1

Leuctra spp.

Amphinemura sp.

Nemoura spp.

s2

Leuctra spp.

Nemoura spp.

Brachyptera seticornis

Protonemura spp.

Chloroperla tripunctata
L1

Nemoura spp.

Leuctra spp.
L2

Leuctra spp.

Nemoura spp.

Amphinemura sp.

Protonemura spp.
SC

Baetis spp.

Leuctra spp.

Amphinemura sp.

Brachyptera seticornis

Chloroperla tripunctata
13

Leuctra spp.

Limnius perrisi

Baetis spp.

Esolus angustatus

Protonemura spp.

Amphinemura sp.

Elmis spp.

Nemoura spp.

\section{R3}

Baetis spp.

Leuctra spp.

Amphinemura sp.

Esolus angustatus

Habroleptoides modesta

Limnius perrisi

Protonemura spp.
Baetis spp.

Leuctra spp.

Limnius perrisi

Esolus angustatus 
Tab. 28 (Forts.)

R2

Baetis spp.

Leuctra spp.

Limnius perrisi

Gammarus pulex

Esolus angustatus

Elmis spp.
R1

Baetis spp.

Gammarus pulex

Amphinemura sp.

Leuctra spp.

Limnius perrisi

den Plecopteren-Gattungen Leuctra, Nemoura, Protonemura und Amphinemura gestellt werden. Daneben tritt aber an B2 und S2 mit Brachyptera seticornis bzw. Chloroperla tripunctata eine weitere dominante Art auf, die für die stark versauerten Stellen untypisch ist. $L 2$ läßt sich nach diesen Kriterien jedoch nicht von den stark versauerten Stellen unterscheiden. Anhand der Gesamtartenzahl wird der Unterschied zwischen beiden Gruppen ebenfalls deutlich, so daß L2 nach diesem Kriterium dicht an B2 und S2 heranrückt.

An den nicht versauerten Stellen ist Baetis immer eine der Arten mit der höchsten Abundanz. Hinzu kommen hier stets eine oder mehrere ColeopterenArten. Unter den dominanten Arten können sich des weiteren Gammarus pulex und eine zweite Ephemeropteren-Art befinden. An den schwach versauerten Stelien sind sowohl typische Arten der nicht versauerten Stellen als auch Arten der mäßig und stark versauerten anzutreffen. Kennzeichnend ist das Auftreten von Baetis, neben der an S3 und auch SC die in den nicht versauerten Bächen nicht mehr zu den dominanten Arten gehörende Brachyptera seticornis vorkommt. Dies gilt an SC auch für Chloroperla tripunctata. Die Dominanzstruktur an L3 spricht fast ausschließlich für eine Einordnung der Probestelle bei den nicht versauerten Stellen. Als Hinweis auf eine schwache Versauerung kann gewertet werden, daß Nemoura, eine für die mäßig und stark versauerten Bereiche typische Gattung, vorkommt und insbesondere, daß die Gesamtartenzahl deutlich unter der der nicht versauerten Stellen und der von S3 und SC liegt.

Bei einer Analyse im Rahmen einer Gewässerüberwachung wird kaum die Möglichkeit bestehen, die Daten von acht Probenahmen heranziehen zu können. Deshalb ist es angebracht zu überprüfen, welche Aussagen auf der Basis von 
einzelnen Probenahmen bereits gemacht werden können. Die Anzahl der sich hinter den Gattungsnamen verbergenden Arten und deren unterschiedliche Entwicklungsgänge führen im Jahresverlauf zu Dominanzverschiebungen an den Probestellen. Leuctra und Nemoura zeigen zum Beispiel an allen mäßig und stark versauerten Stellen ein Abundanzminimum in den Sommermonaten und hohe Werte vom Herbst bis zum Frühjahr. An den nicht versauerten Stellen tritt Leuctra hingegen das ganze Jahr hindurch mit stärkeren, unregelmäßigen Schwankungen auf. An den stark versauerten Probestellen ist Amphinemura nur in den Sommermonaten zu finden, die restlichen Stellen verzeichnen zusätzlich oder ausschließlich ein Maximum im Herbst. Brachyptera seticornis läßt sich im Benthos nur zwischen Herbst und Frühjahr nachweisen. Zu den stets anzutreffenden Arten gehören die ihren gesamten Entwicklungszyklus im Gewässer durchlaufenden Gammariden und Coleopteren. Aber auch bei ihnen kommt es zu großen Schwankungen der Abundanz im Jahresverlauf.

Eine besondere Problematik ergibt sich bei der korrekten Berücksichtigung der Larven der Gattung Baetis. In den nicht versauerten Gewässern ist Baetis ganzjährig mit einem Abundanzmaximum im Herbst und Winter, teilweise auch im Sommer, anzutreffen. An den schwach versauerten Stellen tritt sie nur in einzelnen Jahren ganzjährig auf. Im Winter und Frühjahr kommt es stets zu einem starken Abundanzrückgang, der bis zum oder fast bis zum völligen Verschwinden der Gattung führt. Die mäßig versauerten Probestellen zeigen ein ähnliches Bild wie die schwach versauerten. Der Zeitraum des Auftretens der Gattung im Gewässer ist jedoch in der Regel deutlich kürzer. In den Sommermonaten kann Baetis an allen schwach und mäßig versauerten Stellen sehr hohe Dominanzen vor allem durch Baetis vernus erreichen, bei deren Eientwicklung es in den Wintermonaten zu einer Diapause kommt, wodurch die Art in den Wintermonaten vor niedrigen $\mathrm{pH}-$ Werten geschützt ist (vgl. Kap. 5.3.3). Außerdem ist im Hinblick auf solche für die Beurteilung wichtigen einzelnen Arten zu beachten, daß noch sehr wenig bekannt ist über die Zusammenhänge zwischen Häufigkeit und Intensität von episodischen Versauerungen an mäßig und schwach versauerten Stellen und die Reaktion der einzelnen Arten hierauf. Werden einzelne Säureschübe noch relativ gut toleriert, kommt es bei Wiederholungen in kürzeren Abständen oder einer längeren Belastungsdauer zu einer sehr starken Erhöhung der Mortalität (MERRETT et al. 1991). Der von Larven von Baetis vernus tolerierte minimale $\mathrm{pH}$-Wert von 4,3 an S2 während einer Hochwasserwelle führte so im Juli 1988 als singuläres Ereignis zu keinem Rückgang der 
Benthos-Besiedlungsdichten bei Baetis. Dies erschwert eine Beurteilung der Versauerungssituation an den meisten Stellen in starkem Umfang.

Eine korrekte Beurteilung ist daher das ganze Jahr hindurch nur an den stark versauerten Stellen sichergestellt. Um auch die leichteren Versauerungsgrade erfassen und unterscheiden zu können, bieten nach den Untersuchungsergebnissen die Frühjahrsmonate März und April die besten Chancen, da zum einen die Gewässerversauerung in den Wintermonaten durch die in höherer Frequenz auftretenden Säurewellen einen größeren Einfluß auf die Zusammensetzung der Makrofauna ausübt und zum anderen zu diesem Zeitpunkt die meisten Insektenlarven so weit entwickelt sind, daß sie sich gut bestimmen lassen. Die Untersuchungen versauerter Gewässer in Wales erbrachte ebenfalls die größten Differenzierungen der biozönotischen Strukturen in den Frühjahrsmonaten (WADE et al. 1989).

Durch die Analyse der bei den Benthosaufsammlungen in den Monaten März bzw. April der einzelnen Untersuchungsjahre gewonnenen Daten kann ebenso wie auf der Grundlage des Gesamtuntersuchungszeitraums meistens eine Klassifizierung der Probestellen nach ihrem Versauerungsgrad durchgeführt werden (Tab. 29). An allen stark versauerten Stellen sind zu diesem Zeitpunkt insgesamt nur 3 - 4 Arten zu finden. Als dominante Arten treten grundsätzlich nur Leuctra und Nemoura sowie meist auch Protonemura auf. An den mäßig versauerten Stellen ist neben diesen drei Arten immer auch eine andere Art aus der Gruppe Amphinemura, Brachyptera seticornis, Chloroperla tripunctata, Siphonoperla torrentium unter den dominanten Arten zu finden. Außerdem ist mit 5 - 8 Arten eine höhere Artenzahl festzustellen. Die nicht versauerten Probestellen sind in jedem Jahr gut durch die an ihnen vorkommenden 4 - 5 Ephemeropteren-Arten sowie die meist sehr zahlreich auftretenden Coleopteren zu erkennen. Auch die Gesamtartenzahl liegt deutlich über der der versauerten Gewässer. Schwierig kann die Unterscheidung zwischen den schwach und den mäßig versauerten Stellen sein, da sich hier zwischen den Probenahmen größere Unterschiede ergeben, die eine eindeutige Zuordnung nicht immer möglich machen. Aufgrund häufig wechselnder Intensität und insbesondere Dauer von Säureschüben ist zwischen diesen beiden Gruppen auch von der hydrochemischen Seite eine Abgrenzung für kürzere Zeiträume relativ schwierig. Die schwach versauerten Stellen weisen aber tendenziell höhere Artenzahlen auf. Es ist mit Baetis maximal eine Ephemeropteren-Art zu finden. Deren Auftreten kann von Jahr zu Jahr unterschiedlich sein. Dies trifft auch auf die mäßig versauerte Stelle B2 zu. 
Tab. 29: Eudominante und dominante Taxa des Benthos im März bzw. April der Untersuchungsjahre

1. "Stark versauert"

\subsection{6}

S1

Leuctra
Protonemura
Nemoura

B1

$$
\begin{aligned}
& \text { Leuctra } \\
& \text { Nemoura }
\end{aligned}
$$

L1 Nemoura

Leuctra

M Leuctra

Nemoura

Protonemura
$03 . / 04.87$

Protonemura

Leuctra

Nemoura

Leuctra

Nemoura

Protonemura

Nemoura

Leuctra

Leuctra

Protonemura

Nemoura
03.88

Protonemura

Leuctra

Nemoura

2. "Mäßig versauert"

04.86

B2

Leuctra
Nemoura
Protonemura
Amphinemura

S2

Leuctra
Nemoura
Protonemura
C. tripunctata

L2

Leuctra
Nemoura
Amphinemura

$03 . / 04.87$

Leuctra

Amphinemura

Nemoura

Protonemura

B. seticornis

Leuctra

S. torrentium

Nemoura

Protonemura

Protonemura

Amphinemura

Leuctra

Nemoura
03.88

\section{Leuctra}

Protonemura

Nemoura

S. torrentium

3. "Schwach versauert"

\subsection{6}

S3
Leuctra
C. tripunctata
Amphinemura
Nemoura
Protonemura

$03 . / 04.87$

Leuctra

S. torrentium

Nemoura

Protonemura

B. seticornis

Baetis

Amphinemura

\subsection{8}

Leuctra

Protonemura

S. torrentium

B. seticornis 
Tab. 29 (Forts.)

SC

Leuctra
Amphinemura
Nemoura

L3
Leuctra
Limnius
Esolus
Nemoura
Amphinemura
Elmis

4. "Nicht versauert"

\subsection{6}

V1

Leuctra
Limnius
Baetis
Esolus
R. semicol.
Elmis

V2

Leuctra
Protonemura
Esolus
Baetis
Limnius

R3

Leuctra

Esolus

Limnius

Elmis

R2

Limnius
G. pulex
Elmis
Leuctra
Esolus
Baetis
Protonemura

R1
S. torrentium

Leuctra

Baetis

Nemoura

C. tripunctata

Leuctra

Protonemura

Amphinemura

Limnius

03.104 .87

03.88

Leuctra

Esolus

Amphinemura

Baetis

Limnius

Esolus

Leuctra

Amphinemura

D. cephalotes

Protonemura

$\begin{array}{ll}\text { Leuctra } & \text { Baetis } \\ \text { Baetis } & \text { Leuctra } \\ \text { Esolus } & \text { S. torrentium }\end{array}$

R. semicol.

Limnius

Baetis

Leuctra

Leuctra

Baetis

Limnius

R. semicvol.

Protonemura

R. semicol.

Limnius

G. pulex

Baetis

Leuctra

G.pulex

Limnius

Protonemura

Amphinemura 
An L3 fehlen zwar Ephemeropteren, aber hier gehören in beiden Jahren Coleopteren zu den dominanten Arten.

Eine korrekte Eingruppierung würde sich anhand dieser Ergebnisse auch für die 1982 untersuchten Probestellen im Oberlauf der Sieber im Harz ergeben (LESSMANN 1983, HEITKAMP et al. 1985). An der obersten, permanent stark versauerten Stelle sind im März ebenfalls nur Nemoura, Leuctra und Protonemura zu finden gewesen. Die unterhalb gelegenen Stellen, die von den pH-Werten her zum mäßig und schwach versauerten Bereich tendieren, können durch das Auftreten von Brachyptera seticornis bzw. Brachyptera seticornis und Baetis ebenfalls gut eingruppiert werden. Die nicht versauerten Stellen zeichnen sich auch in der Sieber und ihren Zuflüssen durch mehrere Ephemeropteren- und Coleopteren-Arten unter den dominanten Arten aus. Insgesamt bringt eine Benthosaufsammlung nach der Frühjahrsschneeschmelze in den meisten Fällen relativ zuverlässige und ausreichend differenzierende Daten, um die Versauerungsbelastung einer Probestelle beurteilen zu können.

Eine Versauerungs-Klassifizierung anhand von Herbstbeprobungen ist hingegen nur für die permanent stark versauerten und die nicht versauerten Stellen möglich, die sich aufgrund von Artenzahlen und Dominanzstrukturen stets sehr deutlich voneinander unterscheiden. Bei der großen Zahl der Stellen, die im Sommerhalbjahr längere Zeit $\mathrm{pH}-$ Werte im neutralen Bereich aufweisen, werden die im Frühjahr festgestellten biozönotischen Unterschiede kleiner, so daß nur noch zwischen nicht versauerten, periodisch versauerten und permanent stark versauerten Stellen unterschieden werden kann.

Ebenfalls gut eignen sich, wie die fischereikundlichen Untersuchungen ergeben haben, Fische für die Versauerungsindikation. Die vorkommenden Arten können einen ersten wichtigen Hinweis liefern, da sich Groppe und Bachforelle gemeinsam nur in den nicht versauerten Bächen nachweisen lassen. Das Fehlen der Groppe an R1 zeigt aber auch, daß neben der Versauerung noch andere Gründe für die Verbreitung dieser Art entscheidend sein können. Vorkommen und Analyse der Struktur der Bachforellenpopulationen anhand von Altersaufbau und Besiedlungsdichten geben an allen untersuchten Stellen gut den Versauerungszustand des Gewässers wieder. Fische fehlen an allen stark versauerten Probestellen. Populationen mit einem normalen Altersaufbau und hoher Besiedlungsdichte sind nur in den nicht versauerten Bereichen und an $\mathrm{L} 3 \mathrm{zu}$ finden. Einen gestörten Altersaufbau zeigen die schwach versauerten Bereiche der Probestel- 
len S3, SC und L2, wobei nur an S3 in einzelnen Jahren Jungfische festgestellt werden konnten. Stets nur wenige ältere Tiere kommen in den mäßig sauren Bereichen von S2 und B2 vor.

Nach diesen Befunden weist L3 die gleiche Populationsstruktur wie die nicht versauerten Stellen und L2 die gleiche wie die schwach versauerten auf. Dies liegt zum einen darin begründet, daß die pH-Messungen an diesen beiden Stellen nur Säureschübe mit relativ geringer Intensität und vor allem Frequenz erbracht haben, wodurch sie bereits eine Zwischenstellung zwischen den pH-Gruppen einnehmen, zum anderen müssen bei der Betrachtung nur einer Art deren Toleranzgrenzen die Eingruppierung bestimmen. Unter diesen Gesichtspunkten ergibt sich eine sehr gute Übereinstimmung zwischen den verschiedenen Versauerungsgruppen und der Reaktion der Bachforellenpopulationen, die die Art zu einem wichtigen Indikator der Gewässerversauerung machen.

Vergleicht man die beiden sich von den abiotischen Bedingungen her sehr ähneinden Stellen S3 und SC miteinander, erstaunt es, daß aufgrund der Analyse der Emergenz und auch des Makrozoobenthos SC eine Stelle ist, die bereits sehr stark zu den nicht versauerten Probestellen tendiert, auf anderen Seite aber die Bachforellenpopulation deutlich schwächer entwickelt ist als an S3 und hier im Gegensatz zu S3 auch bei keiner Befischung ein Jungfisch gefangen wurde. Eine mögliche Erklärung hierfür liefern die gemessenen Konzentrationen an Gesamt-Aluminium, bei denen SC mit maximal $966 \mu \mathrm{g} / \mathrm{l}$ deutlich über den maximal $450 \mu \mathrm{g} / \mathrm{l}$ liegt, die an $\mathrm{S} 3$ gemessen wurden. Beide Stellen haben einen mittleren Anteil an monomeren anorganischen Spezies von etwa $70 \%$. Dies spricht dafür, daß die Aluminium-Konzentrationen im $\mathrm{pH}$ Bereich zwischen 5 und 6 bei der Bachforelle von wesentlich größerer Bedeutung sind als bei den Invertebraten (vgl. Kap. 6.2).

GEBHARDT et al. (1989) kommen aufgrund der Ergebnisse ihrer Untersuchungen an Bachforellenpopulationen des Schwarzwaldes zur Einteilung von vier Versauerungsklassen, die anhand der Verbreitungs- und Populationsstrukturbefunde der Bachforelle definiert werden. Danach sind R1 und $V 1$ als nicht versauert anzusehen, R2, L3, V2, R3 und unter Einschränkungen auch S3 als mäßig versauert, L2, SC, S2 und B2 als stark versauert sowie $S 1, L 1, B 1$ und $M$ als übermäßig stark versauert. Diese Gruppeneinteilung läßt sich für die im Harz untersuchten Fließgewässer im Hinblick auf die Ergebnisse der Untersuchungen an den Bachforellen, insbesondere auch anhand der Untersuchungen der 
Makroinvertebraten und von den pH-Gängen der Probestellen her nur teilweise nachvollziehen.

Eine zusammenfassende Betrachtung der Frage der Möglichkeiten der biologischen Indikation der Gewässerversauerung muß anhand der an den 15 Probestellen im Harz gewonnenen Ergebnisse zu dem Schluß gelangen, daß die biologische Indikation sowohl über die Makroinvertebraten als auch über die Fische grundsätzlich möglich ist. Vor einer Übertragung auf andere Gebiete und auch auf Fließgewässer mit anderen hydrographischen Bedingungen im Harz selbst bedarf es aber noch der Klärung einer Reihe von offenen Fragen und erheblicher Ergänzungen.

Die Definition verschiedener Versauerungsgrade sollte anhand hydrochemischer Faktoren erfolgen. Der pH-Wert ist aufgrund der mit inm durch hohe Korrelationen verbundenen zahlreichen weiteren Parameter der naheliegendste und auch geeignete Faktor, um eine Klassifizierung der Versauerung vorzunehmen. Auf der Grundlage der mittleren Konzentrationen und der Frequenz und Intensität von Konzentrationsänderungen der Wasserstoffionen können bei den untersuchten Bächen vier verschiedene Versauerungsgrade unterschieden werden, die per definitionem als "nicht versauert", "schwach versauert", "mäßig versauert" und "stark versauert" bezeichnet werden. Die vier Klassen stehen für folgende $\mathrm{pH}$ Bereiche:

$\begin{array}{ll}\text { pH-Mittelwert } & >6,8 \\ \text { Minimum-pH } & >6,0\end{array}$

2 "Schwach versauert":

pH-Mittelwert

$6,8-6,1$

Minimum-pH

$5,7-4,9$

3 "Mäßig versauert":

pH-Mittelwert

$6,0-5,0$

Minimum-pH

$5,3-4,0$

4 "Stark versauert":

pH-Mittelwert

$<5,0$

Minimum-pH

$<4,4$

Während die nicht versauerten Stellen ganzjährig pH-Werte im neutralen Bereich haben, treten an schwach versauerten Stellen periodische $\mathrm{pH}$-Absenkungen mit allerdings noch relativ geringer Intensität und Frequenz auf. Die weitaus 
überwiegende Zeit des Jahres liegen die $\mathrm{pH}$-Werte im neutralen Bereich. An mäßig versauerten Stellen machen sich die $\mathrm{pH}$-Absenkungen aufgrund größerer Intensität und Frequenz bereits auch bei den mittleren pH-Werten bemerkbar. An stark versauerten Stellen sind die Wasserstoffionenkonzentrationen ganzjährig sehr hoch. An einigen dieser Stellen kann es jedoch zeitweilig zu kurzen Perioden mit neutralen pH-Werten kommen.

Anhand der biozönotischen Strukturen des Makrozoobenthos und/oder durch fischereikundliche Untersuchungen lassen sich die Probestellen entsprechend der herrschenden $\mathrm{pH}$-Bedingungen mehr oder weniger einer der Klassen zuordnen, wobei es auch Übergangsbereiche geben kann und Unterschiede zwischen einzelnen Jahren möglich sind. Für die Benthosuntersuchungen eignen sich insbesondere die Frühjahrsmonate März und April. Bei fischereikundlichen Untersuchungen sind im Herbst die aussagekräftigsten Ergebnisse zu erwarten. In Bezug auf die einzelnen Versauerungsgrade ergeben sich zu diesen Zeitpunkten bei der Besiedlung durch Mollusken, Amphipoden, Ephemeropteren, Plecopteren und Coleopteren sowie bei den Fischpopulationen folgende Strukturen:

1. "Nicht versauert":

hohe Abundanzen mindestens einer Ephemeropteren-Art und/oder einer Coleopteren-Art; Gesamtartenzahl > 10, darunter mindestens 4 Ephemeropteren-Arten, meistens Vorkommen von Ancylus fluviatilis und Gammariden. Bachforelle mit normalem Altersaufbau und in relativ großer Dichte, meistens Vorkommen der Groppe.

\section{2. "Schwach versauert":}

hohe Abundanzen von Leuctra, ansonsten Zusammensetzung der dominanten Arten der Benthoszönose sehr unterschiedlich. Gesamtartenzahl 8 - 11, Ephemeropteren können durch Baetis vertreten sein. Bachforelle mit gestörtem Altersaufbau durch Fehlen der Jungfische in jedem oder in den meisten Jahren, Besiedlungsdichten niedrig; Groppe fehlt.

\section{3. "Mäßig versauert":}

hohe Abundanzen von Leuctra, Nemoura, Protonemura sowie einer weiteren Plecopteren-Art aus der Gruppe Amphinemura, Brachyptera seticornis, Chloroperla tripunctata, Siphonoperla torrentium; Gesamtartenzahl 5 - 8, keine Ephemeropteren. Nur wenige größere Bachforellen in geringer Dichte. 
4. "Stark versauert":

hohe Abundanzen ausschließlich von Leuctra, Nemoura und meist Protonemura;

Gesamtartenzahl 3 - 4, keine Ephemeropteren. Fische fehlen. 


\section{Danksagung}

Daß die Forschungsarbeiten in dem dargestellten großen Umfang durchgeführt werden konnten, ist einer ganzen Reihe von Frauen und Männern zu verdanken, die zum Gelingen der Untersuchungen in vielfältigster Weise beigetragen haben. Für die gewährte großzügige Unterstützung möchte ich insbesondere Prof. Dr. Ulrich Heitkamp, Prof. Dr. Jürgen Schneider, Dr. Jörg Matschullat, Prof. Dr. Dr. Bernhard Ulrich, Dr. Peter Groth, Dipl.-Geogr. Ralf Trapphoff, Dr. Ulrich Siewers, Dipl.-Geol. Abdul Hanan Roostai, Dr. Michael Hauhs und Dr. Gabi Dietze herzlich danken. 


\section{Zusammenfassung}

Zwischen 1986 und 1989 wurden im Rahmen eines interdisziplinären Forschungsvorhabens limnologische Untersuchungen zu den Auswirkungen der Gewässerversauerung auf Fließgewässerbiozönosen im Harz durchgeführt. Für die Untersuchungen wurden im Epirhithral von sechs über den Westharz verstreut liegenden Bächen 15 von direkten anthropogenen Einflüssen freie und von Lage und Struktur her vergleichbare Probestellen ausgewählt, an denen aufgrund der geologischen Bedingungen mit einer unterschiedlichen Empfindlichkeit gegenüber der Versauerungsbelastung zu rechnen war. Die in der Sösemulde gelegenen jeweils drei Probestellen an der nicht versauerten Alter Riefensbeek und der versauerten Großen Söse wurden im Rahmen einer Schwerpunktuntersuchung zwischen 1986 und 1989 einer intensiveren Bearbeitung unterzogen als die 1986 und 1987 untersuchten Stellen der Nebenuntersuchungsbäche. Die vorliegende Arbeit gibt die Ergebnisse der mittels Benthosbeprobungen und Emergenzerfassungen durchgeführten Untersuchungen der Makrobenthosgruppen Mollusca, Amphipoda, Ephemeroptera, Plecoptera und Coleoptera sowie die durch Elektrobefischungen gewonnenen Ergebnisse der fischereikundlichen Untersuchungen wieder.

Der Harz gehört zu den am stärksten durch Luftschadstoffe belasteten Gebieten Mitteleuropas, was in allen Gewässern zu einer hohen Sulfat- und Nitratkonzentration führt. In vielen der nur geringe Konzentrationen an basischen Kationen aufweisenden, elektrolytarmen Gewässern ist dies mit einer Versauerung verbunden. Die Analyse der umfangreichen $\mathrm{pH}-\mathrm{Messungen}$ ermöglichte eine Unterteilung der Probestellen in vier Gruppen, die Unterschiede in Bezug auf Intensität und Frequenz der pH-Absenkungen zeigen:

- nicht versauerte Gewässerabschnitte mit nur geringen Schwankungen der Wasserstoffionenkonzentration im permanent neutralen Bereich,

- schwach versauerte Gewässerabschnitte mit mittleren $\mathrm{pH}$-Werten im neutralen Bereich und gelegentlichen periodischen Säureschüben mit pH-Absenkungen bis etwa $\mathrm{pH}$,

- mäßig versauerte Gewässerabschnitte mit mittleren pH-Werten im schwach sauren Bereich $(\mathrm{pH} 5-6)$ und regelmäßigen periodischen $\mathrm{pH}$-Absenkungen mit $\mathrm{pH}-$ Werten $<5$, 
- stark versauerte Gewässerabschnitte mit mittleren pH-Werten im stark sauren Bereich $(\mathrm{pH}<5)$, die permanent stark versauert sind oder nur kurze Zeit des Jahres neutrale $\mathrm{pH}-$ Werte aufweisen.

$\mathrm{Zu}$ den wichtigsten mit den pH-Werten korrelierten hydrochemischen Parametern gehören die Säurekapazität sowie die Konzentrationen an Aluminium, Calcium, Magnesium, Cadmium und Blei. Speziierungsanalysen des Aluminiums zeigen, daß der Anteil monomerer anorganischer Species mit zunehmenden Konzentrationen an gelöstem organischem Kohlenstoff an den stark versauerten Stellen abnimmt. Eine Cluster-Analyse der Daten der hydrochemischen Parameter führt zu einer Aufteilung der Probestellen in zwei Hauptgruppen mit den nicht versauerten Stellen auf der einen und den versauerten Probestellen auf der anderen Seite.

Im Rahmen der biologischen Bearbeitungen konnten insgesamt 76 Arten und höhere Taxa nachgewiesen werden, wobei die artenreichsten Gruppen die Plecopteren mit 35 Arten und die Ephemeropteren mit 19 Arten sind. Für alle Gruppen ergibt sich eine hohe Abhängigkeit der an einer Probestelle vorkommenden Artenzahl vom Grad der Versauerung, wobei sich Gastropoden und Amphipoden nur in den nicht versauerten Gewässern nachweisen lassen und Ephemeropteren mit zunehmender Versauerung den stärksten Artenrückgang bis zum Fehlen in den stark versauerten Bereichen zeigen. An den stark versauerten Stellen kommen von den untersuchten Tiergruppen in der Regel nur noch Plecopteren und Coleopteren vor.

Die autökologische Auswertung der Untersuchungsergebnisse erbrachte entsprechend sehr niedrige bis niedrige Säuretoleranzen für die Gammariden, die meisten der Ephemeropteren-Arten und den größten Teil der Setipalpia unter den Plecopteren.

Im Benthos sind sehr hohe Dominanzen einzelner Plecopteren-Arten an den stark versauerten Probestellen zu finden, wohingegen sich die Dominanzstrukturen der mäßig und schwach versauerten Stellen zunehmend denen der nicht versauerten Stellen annähern, die die am stärksten ausgeglichenen Dominanzstrukturen aufweisen, was auch in einem Anstieg der Diversitätsindices zum Ausdruck kommt. In Bezug auf die Besiedlungsdichten weisen die nicht versauerten Stellen überwiegend höhere Gesamtabundanzen auf als die versauerten Gewässerbereiche. An den stark versauerten Stellen 
können Massenentwicklungen einzelner Plecopteren-Arten beobachtet werden, wobei die Besiedlungsdichten die der nicht versauerten Stellen weit überschreiten.

An der Emergenz haben die Ephemeropteren unabhängig vom Versauerungsgrad nur einen Anteil von maximal $20 \%$. Während die Ephemeropteren an den nicht versauerten Stellen bereits im Frühjahr höhere Abundanzen zeigen, tritt die Gruppe an versauerten Stellen erst im Sommer und Herbst auf. Die Gesamtemergenzdichte erreicht an drei der vier stark versauerten Stellen die höchsten Werte. Zwischen mäßig, schwach und nicht versauerten Stellen ergeben sich bei der Emergenzdichte keine Unterschiede. Während die Dominanzstrukturen in den stark versauerten Bereichen stets von nur wenigen eudominanten Arten bestimmt werden, ist mit abnehmender Versauerung eine deutliche Tendenz zu ausgeglicheneren Strukturen zu verzeichnen, wobei aber auch an nicht versauerten Stellen wenige Arten eine sehr hohe Dominanz erreichen können. Im Vergleich der Untersuchungsjahre weisen die einzelnen Stellen nur geringe Veränderungen der Dominanzstrukturen auf. Insgesamt ist eine relativ große Übereinstimmung des Spektrums dominanter Arten innerhalb der verschiedenen Versauerungsgrade festzustellen. Entsprechend ergibt ein biozönotischer Ähnlichkeitsvergleich deutlich getrennte biozönotische Strukturen zwischen versauerten und nicht versauerten Gewässerabschnitten, wobei die stark versauerten Probestellen untereinander die größte Ähnlichkeit aufweisen. Abhängig vom Grad der Versauerung lassen sich relativ charakteristische biozönotische Strukturen nachweisen. Für die Diversität errechnen sich die niedrigsten Werte für die stark versauerten Gewässerabschnitte, wohingegen die mäßig und schwach versauerten Stellen keine grundsätzlichen Unterschiede zu den nicht versauerten Bereichen zeigen.

Ebenfalls eine starke Abhängigkeit vom Grad der Versauerung erbrachten die fischereikundlichen Untersuchungen für die Fischpopulationen. Nur in nicht versauerten Gewässer kann neben der Bachforelle (Salmo trutta fario) die Groppe (Cottus gobio) auftreten, und es können sich normal strukturierte Fischpopulationen mit höheren Dichten entwickeln. In schwach versauerten Gewässern fehlt die Groppe, und die Bachforellen zeigen bei niedrigen Besiedlungsdichten einen gestörten Altersaufbau durch Fehlen der Jungfische in den meisten Jahren. Mäßig versauerte Gewässer weisen nur einen Bestand von wenigen größeren Bachforellen auf. In stark versauerten Bereichen fehlen Fische vollständig. 
Durch die Verknüpfung und Analyse der biologischen und abiotischen Daten läßt sich nachweisen, daß die beobachteten Unterschiede in der Struktur der Benthosbiozönosen und bei den Fischpopulationen weitestgehend auf die Gewässerversauerung zurückzuführen sind, wobei für Veränderungen sowohl erhöhte Wasserstoffionenkonzentrationen und teilweise auch erhöhte Konzentrationen monomeren anorganischen Aluminiums verantwortlich gemacht werden können, als auch indirekte Veränderungen zu beobachten sind. Die Abhängigkeit der biozönotischen Auswirkungen vom Grad der Gewässerversauerung und die relativ charakteristischen Emergenz- und Benthosdominanzstrukturen lassen sich für eine biologische Indikation des Ausmaßes der Versauerung nutzen. 
ALICKE, R. (1974): Die hydrochemischen Verhältnisse im Westharz und ihre Beziehung zur Geologie und Petrographie. - Clausthal-Zellerfeld.

ALLARD, M. \& G. MOREAU (1987): Effects of experimental acidification on a lotic macroinvertebrate community. - Hydrobiologia 144: 37-49.

ANDREAE, H. \& R. MAYER (1989): Einfluß der Bodenversauerung auf die Mobilität von Schwermetallen im Einzugsbereich der Söse-Talsperre im Harz. - DVWK Mitt. 17: 285-292.

APEL, R. \& H. FAUTH (1977): Hydrogeologische Verhältnisse. - Erläuterungen zur Geologischen Karte von Bayern, Bayerisches Geologisches Landesamt, München.

AUBERT, J. (1959): Plecoptera. - Insecta Helvetica Fauna 1, Lausanne.

AVERMÁNN, T. (1990a): Untersuchungen zur Toleranz ausgewählter Fließgewässerorganismen gegenüber Versauerungserscheinungen. - Diplomarbeit Univ. Göttingen.

AVERMANN, T. (1990b): VII. Laborexperimentelle Untersuchungen. - In: HEITKAMP, U. \& D. LESSMANN ( $\mathrm{Hg}$. ), Fallstudie Harz: Auswirkungen der Gewässerversauerung auf Bergbach- und Seebiozönosen. - Berichte des Forschungszentrums Waldökosysteme, Göttingen, Reihe B, Bd. 19: 217-254.

BACKHAUS, K., B. ERICHSON, W. PLINKE, C. SCHUCHARD-FISCHER \& R. WEIBER (1989): Multivariate Analysemethoden. - Berlin, Heidelberg, New York.

BAKER, J.P. \& C.L. SCHOFIELD (1982): Aluminium toxicity of fish in acidic waters. - Water, Air, Soil Poll. 18: 289-309.

BAKER, M.D., W.E. INNISS, C.I. MAYFIELD \& P.T.S. WONG (1983): Effect of acidification, metals and metalloids on sediment microorganisms. - Water Res. 17: 925-930.

BAUER, J. , P. SCHMITT, R. LEHMANN \& T. FISCHER-SCHERL (1987): Untersuchungen zur Gewässerversauerung an der oberen Waldnaab (Oberpfälzer Wald; Nordost-Bayern). - Berichte ANL 1: 139-170.

BAUER, J., R. LEHMANN \& A. HAMM (1988): PH-Wert-Veränderungen an ungepufferten Seen und Fließgewässern durch saure Deposition und ökologische Aspekte der Gewässerversauerung. - In: BAYERISCHE LANDESANSTALT FÜR WASSERFORSCHUNG (Hg.), Gewässerversauerung im nord- und nordostbayerischen Grundgebirge. München.

BELL, H. (1971): Effect of low $\mathrm{pH}$ on the survival and emergence of aquatic insects. - Water Res. 5: 313-319.

BENGTSSON, J. (1988): Autecological studies on Baetis rhodani (PICT.) (Ephemeroptera). - Arch. Hydrobiol. 111: 615-623.

BERTHELEMY, C. (1962): Contribution a I'etude systematique des Elminthidae (Coleopteres). - Bull. Soc. Hist. nat. Toulouse 97: 201-225.

BJÄRNBORG, B. (1983): Dilution and acidification effects during the spring flood of four Swedish mountain brooks. - Hydrobiologia 101:19-26.

BLAB, J., E. NOWAK, W. TRAUTMANN \& H. SUKOPP (1984): Rote Liste der gefährdeten Tiere und Pflanzen in der Bundesrepublik Deutschland. Naturschutz Aktuell 1, Greven.

BLANKE, D. (1988): Ökologische Untersuchungen der Makrobenthon- und Fischfauna des südniedersächsischen Mittelgebirgsbaches der Bewer bei Markoldendorf. - Diplomarbeit Univ. Göttingen.

BLAUME, A. (1990): Kartierung und limnogeologische Untersuchung von Sedimenten aus dem Oderteich (Harz). - Diplomarbeit Univ. Göttingen.

BOHLE, H.W. (1969): Untersuchungen über die Embryonalentwicklung und die embryonale Diapause bei Baetis vernus CURTIS und Baetis rhodani (PICTET)(Baetidae, Ephemeroptera). - Zoolog. Jahrb. Abt. Anat. Ont. 86: 493-557. 
BORG, H. (1983): Trace metals in Swedish natural fresh waters. - Hydrobiologia 101: 27-34.

BRAASCH, D. \& U. JACOB (1976): Die Verwendung der Ephemeropteren (Insecta) der DDR als Indikatoren für die Wassergüte. - Entomol. Nachr. 20: 101-109.

BRAHMER, G. \& K.-H. FEGER (1989): Hydrochemische Bilanzen kleiner bewaldeter Einzugsgebiete des Südschwarzwaldes. - DVWK Mitt. 17: 205211.

BRAUER, M., A. THEURICH \& B. STREIT (1990): Plecopterenbesiedlung in zwei Mittelgebirgsbächen mit unterschiedlichem geologischem Einzugsgebiet. - In: DEUTSCHE GESELLSCHAFT FÜR LIMNOLOGIE ( $\mathrm{Hg}$. ), Erweiterte Zusammenfassungen der Jahrestagung 1988 in Goslar 2: 139-145.

BRAUKMANN, U. (1987): Zoozönologische und saprobiologische Beiträge zu einer allgemeinen regionalen Bachtypologie. - Arch. Hydrobiol. Beih. Ergebn. Limnologie 26.

BREHM, J. \& M.P.D. MEIJERING (1982): Zur Säureempfindlichkeit ausgewählter Süßwasser-Krebse (Daphnia und Gammarus, Crustacea). - Arch. Hydrobiol. 95: 17-27.

BRETTFELD, R. (1987): Der Einfiuß der pH-Wert-Absenkung auf die biologische Struktur eines Bergbachsystems im mittleren Erzgebirge. - Veröff. Naturhist. Museum Schleusingen 2: 57-76.

BROCK, V. \& R. BLUM (1977): Steinfliegen (Plecoptera) in einem Fließgewässer im Harz. - Festschrift 125 Jahre Naturwiss. Verein Goslar: 37-60.

BROWN, D.J.A. (1983): Effect of calcium and aluminium concentrations on the survival of brown trout (Salmo trutta) at low pH. - Bull. Environm. Contam. Toxicol. 30: 582-587.

BRUNKEN, H. (1988): Ausbreitungsdynamik von Noemacheilus barbatulus (LINNAEUS, 1758). - Diss. TU Braunschweig.

BUCK, H. \& H. MERZ (1976): Baetis-Schlüssel (Larven). - Landesanstalt für Gewässerkunde Baden-Württemberg.

BUIKEMA, A.L. \& E.F. BENFIELD (1979): Use of macroinvertebrate life history information in toxicity tests. - J. Fish. Res. Bd. Can. 36: 321.

BÜRK, R. (1979): Vergleichende Untersuchungen zur Wassergüte einiger Bäche im südlichen Odenwald. - Diplomarbeit Univ. Heidelberg.

BURTON, T.M., R.M. STANFORD \& J.W. ALLAN (1985): Acidification effects on stream biota and organic matter processing. - Can. J. Fish. Aquat. Sci. 42: 669-675.

BURTON, T.M. \& J.W. ALLAN (1986): Influence of $\mathrm{pH}$, aluminium, and organic matter on stream invertebrates. - Can. Journ. Fish. Aquat. Sci. 43: 1285-1289.

CAIRNS, J. (1981): Review paper: Biological monitoring, Part VI - future needs. - Water Res. 15: 941.

CARRICK, T.R. (1979): The effect of acid water on the hatching of salmonid eggs. - J. Fish. Biol. 14: 165-172.

CASPERS, N. (1980): Die Emergenz eines kleinen Waldbaches bei Bonn. Decheniana Beih. 23.

CASPERS, N. \& H. STIERS (1977): Beitrag zur Kenntnis der Plecopteren der Eifel (Insecta: Plecoptera). - Decheniana 130: 136-150.

CHUTTER, F.M. (1972): A reappraisel of Needham and Usinger's data on the variability of stream fauna when sampled with a Surber Sampler. Limnol. Ocean. 17: 139-141.

CHUTTER, F.M. \& R.G. NOBLE (1966): The reliability of a method for sampling stream invertebrates. - Arch. Hydrobiol. 62: 95-103.

CORING, E. (1988): Untersuchungen zur Zusammensetzung der Diatomeen-Flora in versauerten und unversauerten Harzbächen. - Staatsexamensarbeit Univ. Göttingen. 
CORING, E. \& U. HEITKAMP (1989): Auswirkungen der Wasserstoffionenkonzentration auf die Zusammensetzung der Diatomeenassoziationen ausgewählter Harzbäche. - DVWK Mitt. 17: 407-412.

CORING, E. (1990) : III. Diatomeenflora. - In: HEITKAMP, U. \& D. LESSMANN (Hg.), Fallstudie Harz: Auswirkungen der Gewässerversauerung auf Bergbach- und Seebiozönosen. - Berichte des Forschungszentrums Waldökosysteme, Göttingen, Reihe B, Bd. 19: 53-82.

COWLING, E.B. (1980): Acid precipitation and its effects on terrestrial and aquatic ecosystems. - Annal. New York Acad. Sci. 338: 540-555.

D'ITRI, F.M. (Hg.) (1982): Acid precipitation - effects on ecological systems. Ann Arbor.

DECKER, C. (1987): Gewässerversauerung in Mitteleuropa, Ursachen und Auswirkungen auf aquatische Ökosysteme - Eine Literaturstudie. Staatsexamensarbeit Univ. Göttingen.

DFG, Deutsche Forschungsgemeinschaft (1982): Schadstoffe im Wasser. I. Metalle. - Forschungsbericht der DFG. Boppard.

DIERSCHKE, H., A. OTTE \& H. NORDMANN (1983): Die Ufervegetation der Fließgewässer des Westharzes und seines Vorlandes. - Naturschutz $u$. Lanschaftspflege in Niedersachsen, Beiheft 4.

DIETZE, G. (1990): The use of flow injection analysis for speciation of aluminium in acid soil water. - Geolog. Landesamt Baden-Württemberg, Freiburg (unveröff.).

DITTMAR, H. (1955): Ein Sauerlandbach. - Arch. Hydrobiol. 50: 305-552.

DRABLOS, D. \& A. TOLLAN (Hg.) (1980): Ecological impact of acid precipitation. - Proc. Int. Conf. Ecol. Impact Acid Precip., Sandefjord, Norway, 11-14 March 1980.

DRISCOLL, C.T. (1985): Aluminium in acidic surface waters: chemistry, transport and effects. - Environm. Health Persp. 63: 93-104.

DRISCOLL, C.T., J.P. BAKER, J.J. BISOGNI \& C.L. SCHOFIELD (1980): Effect of aluminium speciation on fish in dilute acidified waters. - Nature 284: $161-164$.

DWD, Deutscher Wetterdienst (1986-1989): Monatlicher Witterungsbericht. Amtsblatt des Dt. Wetterdienstes.

EDDY, F.B. (1982): Osmotic and ionic regulation in captive fish with particular reference to salmonids. - Comp. Biochem. Physiol. 72B: 125-141.

EGGLISHAW, H.J. (1964): The distributional relationship between the bottom fauna and plant detritus in streams. - Jour. Animal Ecol. 33: 463-476.

EGGLISHAW, H.J. (1968): The quantitative relationship between bottom fauna and plant detritus in streams of different calcium concentrations. - J. Appl. Ecol. 5: 731-740.

ELLENBERG, H. (1986): Vegetation Mitteleuropas mit den Alpen. - Stuttgart.

ELLIOTT, J.M., U.H. HUMPESCH \& T.T. MACAN (1988): Larvae of the British Éphemeroptera: A key with ecological notes. - Freshwater Biol. Ass. Sci. Publ. 49.

ENGBLOM, E. \& P.-E. LINGDELL (1984): The mapping of short-term acidification with the help of biological $\mathrm{pH}$ indicators. - Inst. Freshwater Res. Drottningholm Rep. 61: 60-68.

FAUTH, H., U. SIEWERS \& G. MÜLLER (1983): Cadmium, Uran und Blei in unseren Gewässern. - Bild der Wissenschaft 20: 77-95.

FISCHER-SCHERL, TH., R.W. HOFFMANN, B. KÜGEL, G. MERK, H. MILLER \& H.J. HOFFMANN (1988): Einfluß der Gewässerversauerung auf die Fischfauna. - In: BAYERISCHE LANDESANSTALT FÜR WASSERFORSCHUNG (Hg.), Gewässerversauerung im nord- und nordostbayerischen Grundgebirge. München.

FREUDE, H., K.W. HARDE \& G.A. LOHSE (1971): Die Käfer Mitteleuropas, Band 3. - Krefeld.

FREUDE, H., K.W. HARDE \& G.A. LOHSE (1979): Die Käfer Mitteleuropaś, Band 6. - Krefeld. 
FRIBERG, F., C. OTTO \& B.S. SVENSSON (1980): Effects of acidification on the dynamics of allochthonous leaf material and benthic invertebrate communities in running waters. - In: D. DRABLOS \& A. TOLLAN ( $\mathrm{Hg}$.$) ,$ Ecological impact of acid precipitation. - Proc. Int. Conf. Ecol. Impact Acid Precip., Sandefjord, Norway, 11-14 March 1980: 304-305.

FRIEDRICH, G. \& Ẃ. GÖRTZ (1985): Untersuchungen zum Problem Gewässerversauerung. - LAWA NRW, Jahresbericht 1985, Düsseldorf: 8-13.

FROMM, P.O. (1980): A review of some physiological and toxicological responses of freshwater fish to acid stress. - Envir. Biol. Fish. 5: 79-93.

FROST, S., A. HUNI \& W.E. KERSHAW (1971): Evaluation of a kicking technique for sampling stream bottom fauna. - Can. Jour. Zool. 49: 167173.

FÜHRER, H.-W., H.-M. BRECHTEL, H. ERNSTBERGER \& C. ERPENBECK (1988): Ergebnisse von neuen Depositonsmessungen in der Bundesrepublik Deutschland und im benachbarten Ausland. - DVWK Mitteilungen 14: 1122.

GARDEN, A. \& R.W. DAVIES (1989): Decomposition of leaf litter exposed to simulated acid rain in a buffered lotic system. - Freshwater Biol. 22: 3344.

GAUMERT, D. (1981): Süßwasserfische in Niedersachsen, Arten und Verbreitung als Grundlage für den Fischartenschutz. - Schriftenreihe des Nds. Min. für Ernähr., Landw. u. Forsten, Hannover. Wolfenbüttel.

GAUMERT, D. (1986): Kleinfische in Niedersachsen, Hinweise zum Artenschutz. - Mitt. Nds. Landesamt f. Wasserwirtschaft 4, Hildesheim.

GEBHARDT, $H$. , M. LINNENBACH, R. MARTHALER, A. NESS \& $H$. SEGNER (1989): Die Bachforelle (Salmo trutta f. fario) - ein Bioindikator für die Gewässerversauerung. - Fischökologie 1: 1-21.

GELLERT, J.F. (1958): Grundzüge der physischen Geographie von Deutschland. - Berlin.

GLÖER, P., C. MEIER-BROOK \& O. OSTERMANN (1980): Süßwassermollusken. - Dt. Jugendbund für Naturbeobachtungen, Hamburg.

GOLL, A. (1985): Öko-Porträts Groppe und Elritze. - NVN, BSH, Beilage zu "natur", München.

GÖRTZ, W., H. ANNA \& S. WALDSTÄDT (1985): Metallaustrag in Fließgewässern induziert durch anthropogene Säurebildner. - FKG Seminar Berlin 9.10.5.1985.

GRASHOF, H. (1972): Ephemeroptera, Plecoptera und Trichoptera aus Harzer Fließgewässern. - Diplomarbeit Univ. Gießen.

GROTH, P. \& R. TRAPPHOFF (1988): Exkursionsführer zur Jahrestagung der Deutschen Gesellschaft für Limnologie in Goslar. - Harzwasserwerke, Langelsheim.

HAASE, H., M. SCHMIDT \& J. LENZ (1970): Der Wasserhaushalt des Westharzes. Hydrologische Untersuchungen 1941-1965. - Veröff. Nds. Inst. Landesentw., Göttingen, Reihe A, 95.

HAINES, T.A. (1981): Acidic precipitation and its consequences for aquatic ecosystems. - Trans. Am. Fish. Soc. 110: 669-707.

HALL, R.J., C.T. DRISCOLL \& G. E. LIKENS (1987): Importance of hydrogen ions and aluminium in regulating the structure and function of stream ecosystems: an experimental test. - Freshwater Biol. 18: 17-43.

HALSBAND, E. \& I. HALSBAND (1975): Einführung in die Elektrofischerei. Berlin.

HARMANEN, M. (1980): Der Einfluß saurer Gewässer auf den Bestand der Ephemeriden- und Plecopterenfauna. - Gew. Abwässer 66/67: 130-136.

HARRIMAN, R. \& B.R.S. MORRISON (1982): Ecology of streams draining forested and non-forested catchments in the area of central Scotland subject to acid precipitation. - Hydrobiologia 88: 251-263. 
HAUHS, M. (1985a): Wasser- und Stoffhaushalt im Einzugsgebiet der Langen Bramke (Harz). - Ber. Forschungszentrum Waldökosysteme/Waldsterben Univ. Göttingen 17.

HAUHS, M. (1985b): Der Einfluß des Waldsterbens auf den Zustand von Oberflächengewässern. - Z. dt. geol. Ges. 136: 585-597.

HAUHS, M. (1985c): Die Beziehung zwischen saurer Deposition, Bodenversauerung, Waldsterben und Gewässerversauerung im Einzugsgebiet der Langen Bramke (Harz). - In: NATIONALPARKVERW. BAYER. WALD (Hg.), Wald und Wasser, 5. Tagungsbericht: 531-570.

HAUHS, M., K. ROST-SIEBERT, G. RABEN, T. PACES \& B. VIGERUST (1989): Summery of European data. - In: J.L. MALANCHUK \& J. NILSSON (Hg.), The role of nitrogen in the acidification of soils and surface waters. Miljorapport 10.

HAVAS, M. \& T.C. HUTCHINSON (1982): Aquatic invertebrates from the Smoking Hills, N.W.T.: Effects of pH and metals on mortality. - Can. J. Fish. Aquat. Sci. 39: 890-903.

HAVAS, M. \& T.C. HUTCHINSON (1983): Effect of low pH on the chemical composition of aquatic invertebrates from tundra ponds at Smoking Hills, N.W.T., Canada. - Can. J. Zool. 61: 242-249.

HAVAS, M., T.C. HUTCHINSON \& G.E. LIKENS (1984): The effects of low $\mathrm{pH}$ on sodium regulation in two species of Daphnia. - Can. J. Zool. 62: 1965-1970.

HAVAS, M. \& G.E. LIKENS (1985): Changes in Na influx and outflux in Daphnia magna (STRAUS) as a function of elevated Al concentrations in soft water at low pH. - Proc. Nat. Acad. Sci. 82: 7345-7349.

HEINRICHS, H., B. WACHTENDORF, K.H. WEDEPOHL, B. RÖSSNER \& $G$. SCHWEDT (1986): Hydrogeochemie der Quellen und kleineren Zuflüsse der Sösetalsperre. - Newes Jahrbuch Miner. Abh. 156: 23-62.

HEITKAMP, H., D. LESSMANN \& C. PIEHL (1985): Makrobenthos, Moos- und Interstitialfauna des Mittelgebirgsbachsystems der Sieber im Harz (SüdNiedersachsen). - Arch. Hydrobiol./Suppl. 70: 279-364.

HEITKAMP, H. \& D. LESSMANN (Hg.) (1990): Fallstudie Harz: Auswirkungen der Gewässerversauerung auf Bergbach- und Seebiozönosen. - Berichte des Forschungszentrums Waldökosysteme, Göttingen, Reihe B, Bd. 19.

HEITKAMP, U. \& J. WILLERS (1990): VIII. Zooplankton und Zoobenthon der Seen. - In: HEITKAMP, U. \& D. LESSMANN (Hg.), Fallstudie Harz: Auswirkungen der Gewässerversauerung auf Bergbach- und Seebiozönosen. Berichte des Forschungszentrums Waldökosysteme, Göttingen, Reihe B, Bd. 19: 255-291.

HEITKAMP, H., D. LESSMANN, J. ROMMELMANN, R. RÜDDENKLAU \& J. WULFHORST (1991): Limnology of a slightly alkaline and an acidified brook in the Harz Mountains (FRG). - Verh. Internat. Verein. Limnol. 24: $1862-1868$.

HENRIKSEN, A. (1979): A simple approach for identifying and measuring acidification of freshwater. - Nature 278: 542-545.

HENRIKSEN, A., O.K. SKOGHEIM \& B. ROSSELAND (1984): Episodic changes in $\mathrm{pH}$ and aluminium speciation kill fish in a Norwegian salmon river. Vatten 40: 255-260.

HERRMANN, J. \& K.G. ANDERSSON (1986): Aluminium impact on respiration of lotic mayflies at low pH. - Water, Air, Soil Poll. 30: 703-709.

HERTER-MAIER, M. \& R. SCHOEN (1988): Verbreitung von Säureschüben in Fließgewässern des Nordschwarzwalds. - Hohenheimer Arbeiten, Gefährdung und Schutz von Gewässern.

HILDREW, A.G., C.R. TOWNSEND, J. FRANCIS \& K. FINCH (1984): Cellulolytic decomposition in streams of contrasting $\mathrm{pH}$ and its relationship with invertebrate community structure. - Freshw. Biol. 14: 323-328. 
HINZE, C. (1971): Erläuterungen zur Geologischen Karte von Niedersachsen, Blatt 4128 Clausthal-Zellerfeld. - Nas. Landesamt für Bodenforschung, Hannover.

$\mathrm{HOCH}$, J. (1982): Der Fischbestand eines nicht bewirtschafteten, mineralarmen Mittelgebirgsbaches (Große Ohe). - Österreichs Fischerei 35: 48-55.

HÖLL, K. (1986): Wasser: Untersuchung, Beurteilung, Aufbereitung, Chemie, Bakteriologie, Virologie, Biologie. - 7. Aufl., Berlin, New York.

HOLLAND, D.G. (1972): A key to the larvae, pupae and adults of the British species of Elminthidae. - Freshwater Biol. Ass. Sci. Publ. 26.

HÖVERMANN, J. (1953): Harz. - In: MEYNEN, E., J. SCHMITHÜSEN, J. GELLERT, E. NEEF, H. MÜLLER-MINY \& J. SCHULTZE (Hg.), Handbuch der naturräumlichen Gliederung Deutschlands. - Bad Godesberg.

HUET, M. (1949): Apercu des relations entre la pente et les populations des eaux courantes. - Schweiz. Z. Hydrol. 11: 333-351.

HUMPESCH, U. (1983): Einfluß der Temperatur auf die Dauer der Embryonalund Larvalentwicklung von Wasserinsekten. - Verh. Ges. Ökol. 10: 543549.

HÜSER, R. \& REHFUESS (1988): Stoffdepostion durch Niederschlag in ost- und südbayerischen Waldbeständen. - Forstl. Forschungsber. München 86.

HWW, Harzwasserwerke (1990): Untersuchungsergebnisse im Rahmen des ECEMonitoring-Programms "Gewässerversauerung in der Bundesrepublik Deutschland". - Zentrallabor der HWW, Langelsheim (unveröff.).

HYNES, H.B.N. (1977): A key to the adults and nymphs of British stoneflies (Plecoptera). - Freshwater Biol. Ass. Sci. Publ. 17.

ILLIES, J. (1952): Die Mölle. Faunistisch-ökologische Untersuchungen an einem Forellenbach im Lipper Bergland. - Arch. Hydrobiol. 46: 424-612.

ILLIES, J. (1955): Steinfliegen oder Plecoptera. - In: F. DAHL (Hg.), Tierwelt Deutschlands 43. Jena.

ILLIES, J. (1961): Versuch einer allgemeinen biozönotischen Gliederung der Fließgewässer. - Int. Rev. ges. Hydrobiol. 46: 205-213.

ILLIES, J. (1963): Plecoptera. - In: P. BROHMER (Hg.), Die Tierwelt Mitteleuropas IV. Leipzig.

ILLIES, J. (1978a): Limnofauna Europaea. - Stuttgart.

ILLIES, J. (1978b): Vergleichende Emergenzmessung im Breitenbach 19691976. - Arch. Hydrobiol. 82: 432-448.

ILLIES, J. \& L. BOTOSANEANU (1963): Problemes et methodes de la classification et de la zonation ecologique des eaux courantes, considerees surtout du point de vue faunistique. - Mitt. Intern. Verein. Limnol. 12: 157.

JACOBS, W. \& M. RENNER (1988): Biologie und Ökologie der Insekten. Stuttgart.

JENS, G. (1968): Tauchstäbe zur Messung der Strömungsgeschwindigkeit und des Abflusses. - Dt. Gewkdl. Mitt. 12: 90-95.

JENS, G. (1980): Die Bewertung der Fischgewässer. - Hamburg, Berlin.

JERNELON, A. (1978): General aspects of toxicology. - In: G.C. BUTLER (Hg.), Principles of ecotoxicolgy. New York.

JOHANNESSEN, M. \& A. HENRIKSEN (1978): Chemistry of snow meltwater: Changes in concentration during melting. - Water Resour. Research 14: 615-619.

KARLSSON-NORRGREN, L., W. DICKSON, O. LJUNBERG \& P. RUNN (1986): Acid water and aluminium exposure: gill lesions and aluminium accumulation in farmed brown trout, Salmo trutta L. - J. Fish Diseases 9: 1-9.

KIMMEL, W.G., D.J. MURPHEY, W.E. SHARPE \& D.R. DEWALLE (1985): Macroinvertebrate community structure and detritus processing rates in two southwestern Pennsylvanian streams acidified by atmospheric deposition. - Hydrobiologia 124: 97-102. 
KIMMINS, D.E. (1972): A revised key to the adults of the British species of Ephemeroptera with notes on their ecology. - Freshwater Biol. Ass. Sci. Publ. 15.

KIS, B. (1973): Plecoptera. - Fauna Rep. Social. Romania, Insecta 8.

KLAUSNITZER, B. (1977): Bestimmungstabellen für die Gattungen der aquatischen Coleopteren-Larven Mitteleuropas. - Beitr. Ent. 27: 145-192.

KLOTZEK, F. (1973): Faunistisch-ökologische Untersuchungen an Plecopteren der Einzugsgebiete von Bode und Selke im Harz. - Hercynia N.F. 10: 162-188.

KNIE, J. (1977): Ökologische Untersuchungen der Käferfauna von ausgewählten Fließgewässern des Rheinischen Schiefergebirges. - Decheniana 130: 151-221.

KOLKWITZ, R. \& M. MARSSON (1902): Grundsätze für die biologische Beurteilung des Wassers nach seiner Fauna und Flora. - Mitt. Kgl. Prüfungsanst. Wasservers. Abwasserbes. Berlin 1: 33-72.

KOLKWITZ, R. \& M. MARSSON (1908): Ökologie der pflanzlichen Saprobien. Ber. dt. Bot. Ges. 26a: 505-519.

KOLKWITZ, R. \& M. MARSSON (1909): Ökologie der tierischen Saprobien. Intern. Rev. Hydrobiol. 2: 126-152.

KOTHE, P. (1962): Der Artenfehlbetrag, ein einfaches Gütekriterium und seine Anwendung bei biologischen Vorfluteruntersuchungen. - Dt. Gewkdl. Mitt. 6: 60-65.

LAGLER, K.F. (1978): Capture, sampling and examination of fishes. - In: T. BAGENAL ( $\mathrm{Hg}$.$) , Methods for the assessment of fish production in fresh$ waters. - IBP-Handbook No 3, 3rd ed., Oxford: 7-47.

LANDA, V. (1969): Jepice - Ephemeroptera. - Fauna CSSR 18.

LAWA, Niedersächsisches Landesamt für Wasser und Abfall (1988): Untersuchung von Niederschlagswasser. - Ber. des LAWA, Hildesheim.

LAWRENCE, G.B., R.D. FULLER \& C.T. DRISCOLL (1986): Aqueous aluminium chemistry in the streams of two upper elevation watersheds in the White Mountains of New Hampshire. - Biogeochemistry 2: 115-135.

LEHRKE, D. \& H. REUSCH (1987): Bisher nachgewiesene Eintagsfliegen des Niedersächsischen Tieflands (Insecta, Ephmeroptera). - Braunschw. Naturkdl. Schr. 2: 685-691.

LEIVESTAD, H., J.P. MUNIZ \& B.O. ROSSELAND (1980): Acid stress in trout from a dilute mountain stream. - In: D. DRABLOS \& A. TOLLAN (Hg.), Ecological impact of acid precipitation. - Proc. Int. Conf. Ecol. Impact Acid Precip., Sandefjord, Norway, 11-14 March 1980.

LELEK, A. (1961): Fische des Rokytna-Flusses. - Diss. Tschech. Akad. Wiss. Brno (in tschech.).

LENHART, B. \& C. STEINBERG (1984a): Limnochemische und limnobiologische Auswirkungen der Versauerung von kalkarmen Oberflächengewässern. Informationsber. Bayer. Landesamt $f$. Wasserwirtschft 4/84, München.

LENHART, B. \& C. STEINBERG (1984b): Auswirkungen der Versauerung auf Fische. - Fischer \& Teichwirt 35: 298-302.

LESSMANN, D. (1983): Faunistisch-ökologische Untersuchungen an der Makrofauna der Sieber und ihrer Zuflüsse, eines Mittelgebirgsbachsystems im Harz. - Diplomarbeit Univ. Göttingen.

LESSMANN, D. \& U. HEITKAMP (1990): Auswirkungen der Gewässerversauerung auf die Fischpopulationen in Bergbächen des Harzes. - In: DEUTSCHE GESELLSCHAFT FÜR LIMNOLOGIE (Hg.), Erweiterte Zusammenfassungen der Jahrestagung 1988 in Goslar 2: 65-74.

LINKERSDÖRFER, S. \& P. BENECKE (1987): Auswirkungen von sauren Depositionen auf die Grundwasserqualität in bewaldeten Gebieten. Eine Literaturstudie. - UBA Materialien 4/87, Berlin. 
LUBIENIECKI, B. \& L. STEINBERG (1985): Der Einfluß des Sauren Regens auf die Embryonalentwicklung der Regenbogenforelle (Salmo gairdneri RICHARDSON) und auf die natürliche Entwicklung der Bachforelle (Salmo trutta f. fario) in ausgesuchten Bächen des Sauerlandes. - Der Fischwirt 7/8: 51-58.

LUBIENIECKI, B. \& L. STEINBERG (1987): Die Auswirkungen von anthropogenen Gewässerversauerungen auf die Ichthyofauna insbesonders der Bachforelle (Salmo trutta f. fario) in ausgesuchten Mittelgebirgsbächen. Der Fischwirt 37: 25-38.

MACKAY, R.J. \& K.E. KERSEY (1985): A preliminary study of aquatic insect communities and leaf decomposition in acid streams near Dorset, Ontario. - Hydrobiologia 122: 3-11.

MALESSA, V. \& B. ULRICH (1989): Beitrag zum Einfluß der Bodenversauerung auf den Zustand der Grund- und Oberflächengewässer. - DVWK Mitt. 17: 213-219.

MALESSA, V. \& H. ANDREAE (1990): Lage und Ausdehnung der Versauerungsfront in der Verwitterungsdecke Diabas, Grauwacke/Tonschiefer und Quarzit an Beispielen aus der Sösemulde. - Ber. Forschungszentrum Waldökosysteme, Reihe B, Bd. 19.

MALICKY, H. (1978): Der Temperaturgang einiger niederösterreichischer Gebirgsbäche. - Wetter und Leben 30: 170-183.

MALTE, H. (1986): Effects of aluminium in hard, acid water on metabolic rate, blood gas tensions and ionic status in the rainbow trout. - J. Fish. Biol. 29: $187-198$.

MASON, B.J. \& H.M. SEIP (1985): The current state of knowledge on the acidification of surface waters and guidelines for further research. Ambio 14: 45-51.

MATSCHULLAT, J. (1989): Umweltgeologische Untersuchungen zu Veränderungen eines Ökosystems durch Luftschadstoffe und Gewässerversauerung (Sösemulde, Harz). - Göttinger Arbeiten zur Geologie u. Paläontologie 42, Göttingen.

MATSCHULLAT, J., H. HEINRICHS, J. SCHNEIDER \& M. STURM (1987): Schwermetallgehalte in Seesedimenten des Westharzes (BRD). - Chem. Erde 47: 181-194.

MATSCHULLAT, J., H. HEINRICHS, A.H. ROOSTAI, J. SCHNEIDER \& U. SIEWERS (1989): Schwermetallbelastung und Gewässerversauerung im Harz. - DVWK Mitt. 17: 293-300.

MATSCHULLAT, J., H. HEINRICHS, J. SCHNEIDER \& U. SIEWERS (1990): Schwermetalle und Gewässerversauerung - Untersuchungen zum Verhalten von Schadstoffen in bewaldeten Ökosystemen. - Z. dt. geol. Ges. 141: 139-150.

MATTHES, G. (1963): Die Herkunft der Sulfat-lonen im Grundwasser. - Abh. Hess. Landesamt Bodenfor. 35, Wiesbaden.

MATTHIAS, U. (1983): Der Einfluß der Versauerung auf die Zusammensetzung der Bergbachbiozönosen. - Arch. Hydrobiol./Suppl. 65: 407-483.

McCAHON, C.P., D. PASCOE \& C. MCKAVANAGH (1987): Histochemical observations on the salmonids Salmo salar $L$. and Salmo trutta $L$. and Ecdyonurus venosus (Fabr.) following a simulated episode of acidity in an upland stream. - Hydrobiologia 153: 3-12.

MCCAHON, C.P. \& D. PASCOE (1989): Short-term experimental acidification of a Welsh stream: toxicity of different forms of aluminium at low pH to fish and invertebrates. - Arch. Environm. Contam. Toxicol. 18: 233-242.

MEESENBURG, H. \& R. SCHOEN (1988): Auswirkungen saurer Niederschläge auf die Wasserqualität der Haslach im Südschwarzwald. - Hohenheimer Arbeiten, Gefährdung und Schutz von Gewässern.

MEIJERING, M.P.D. (1971): Die Gammarus-Fauna der Schlitzerländer Fließgewässer. - Arch. Hydrobiol. 68: 575-608. 
MEIJERING, M.P.D. \& H.G. PIEPER (1982): Die Indikatorbedeutung der Gattung Gammarus in Fließgewässern. - Decheniana-Beih. 26: 111-113.

MEIJERING, M.P.D. \& H.G. PIEPER (1985): Zur Verbreitung der Gattung Gammarus (Crustacea: Amphipoda) im Fulda-Eder-Abflußgebiet mit besonderer Berücksichtigung der Bachversauerung. - Mittlg. Erg. Stud. Ökolog. Umweltsich. 10: 91-123.

MEINEL, W. \& U. MATTHIAS (1982): Chemisch-physikalische und faunistische Untersuchungen an einem Mittelgebirgsbach (Nieste, Naturpark Kaufunger Wald, Nordhessen). II. Faunistischer Teil. - Philippia V/1: 11-32.

MEINEL, W., U. MATTHIAS \& S. ZIMMERMANN (1985): Ökophysiologische Untersuchungen zur Säuretoleranz von Gammarus fossarum (KOCH). Arch. Hydrobiol. 104: 287-302.

MERRETT, W.J., G.P. RUTT, N.S. WEATHERLEY, S.J. THOMAS \& S.J. ORMEROD (1991): The response of macroinvertebrates to low $\mathrm{pH}$ and increased aluminium concentrations in Welsh streams: multiple episodes and chronic exposure. - Arch. Hydrobiol. 121: 115-125.

MEYNEN, E., J. SCHMITHÜSEN, J. GELLERT, E. NEEF, H. MÜLLER-MINY \& J. SCHULTZE (1953-1962): Handbuch der naturräumlichen Gliederung Deutschlands. - Bad Godesberg.

MOHR, K. (1984): Harz - westlicher Teil. - Sammlung Geolog. Führer 58, Berlin.

MOUNTFORD, M.D. (1962): An index of similarity and its application to classificatory problems. - In: P.W. MURPHY (Hg.), Progress in soil zoology, London: $43-50$.

MÜHLENBERG, M. (1989): Freilandökologie. - Heidelberg, Wiesbaden.

MÜLLER-LIEBENAU, I. (1969): Revision der europäischen Arten der Gattung Baetis LEACH, 1815 (Insecta, Ephemeroptera). - Gew. Abwässer 48/49.

MUNIZ, J.P. \& H. LEIVESTAD (1980): Acidification - effects on freshwater and fish. - In: D. DRABLOS \& A. TOLLAN (Hg.), Ecological impact of acid precipitation. - Proc. Int. Conf. Ecol. Impact Acid Precip., Sandefjord, Norway, 11-14 March 1980: 84-92.

NEVILLE, C.M. \& P.G.C. CAMPBELL (1988): Possible mechanisms of aluminium toxicity in a dilute, acidic environment to fingerlings and older life stages of salmonids. - Water, Air, Soil Poll. 42: 311-327.

NILSSEN, J.P. (1980): Acidification of small watersheds in southern Norway and some characteristics of acidic aquatic environments. - Int. Rev. ges. Hydrobiol. 65: 177-207.

ÖKLAND, J. \& K.A. ÖKLAND (1986): The effects of acid deposition on benthic animals in lakes and streams. - Experimentia 42: 471-486.

ORMEROD, S.J. \& R.W. EDWARDS (1987): The ordination and classification of macroinvertebrate assemblages in the catchment of the River Wye in relation to environmental factors. - Freshw. Biol. 17: 533-546.

ORMEROD, S.J., K.R. WADE \& A.S. GEE (1987a): Macrofloral assemblages in upland Welsh streams in relation to acidity, and their importance to invertebrates. - Freshw. Biol. 18: 545-557.

ORMEROD, S.J., P. BOOLE, C.P. MCCAHON, N.S. WEATHERLEY, D. PASCOE \& R.W. EDWARDS (1987b): Short-term experimental acidification of a Welsh stream: comparing the biological effects of hydrogen ions and aluminium. - Freshwater Biol. 17: 341-356.

OTTO, A. \& U. BRAUKMANN (1983): Gewässertypologie im ländlichen Raum. Schriftenreihe des Bundesmin. Ern., Landw. u. Forsten, Reihe A, 288.

OTTO, C. \& B.S. SVENSSON (1983): Properties of acid brown water streams in South Sweden. - Arch. Hydrobiol. 99: 15-36.

OVERREIN, L.N. (1983): Acid precipitation - an international environmental problem. - Water Sci. Tech. 15: 1-7.

OVERREIN, L., H. SEIP \& A. TOLLAN (1980): Acid precipitation -effects on forest and fish. - Final report of the SNSF-Project, Oslo.

PALISSA, A., E.M. WIEDENROTH \& K. KLIMT (1979): Anleitung zum ökologischen Geländepraktikum. - Potsdam. 
PALUMBO, A.V., P.J. MULHOLLAND \& J.W. ELWOOD (1987): Microbial communities on leaf material protected from macroinvertebrate grazing in acidic and circumneutral streams. - Can. J. Fish. Aquat. Sci. 44: 1064-1070.

PANKOW, W. (1979): Beitrag zur Kenntnis der mitteleuropäischen Arten der Gattung Elmis LATREILLE (Coleoptera, Elminthidae). - Ent. Z. 16: 182191 ,

PAULY, D. (1973): Über ein Gerät zur Vorsortierung von Benthosproben. - Ber. dt. wiss. Komm. Meeresforsch. 22: 458-460.

PECHARSKY, B.L. (1984): Predator-prey interactions among aquatic insects. In: V.H. RESH \& D.M. ROSENBERG (Hg.), The ecology of aquatic insects. New York.

PHILLIPS, J. (1980): Cadmium. - In: E. MERIAN (Hg.), Metalle in der Umwelt. Weinheim, Basel.

PIEHL, C. (1983): Die Besiedlung des hyporheischen Interstitials und der Moospolster eines Mittelgebirgsbachs (Sieber) im Harz. - Diplomarbeit Univ. Göttingen.

PIELOU, E.C. (1975): Ecological diversity. - New York, London, Sydney, Toronto.

PINKSTER, S. (1972): On members of the Gammarus pulex-group (Crustacea, Amphipoda) from Western Germany. - Bijdr. Dierk. 42: 164-191.

PLANAS, D. \& G. MOREAU (1986): Reaction of lotic periphyton to experimental acidification. - Water, Air, Soil Poll. 30: 681-686.

PRENZEL, J. (1986): A numerical scheme for the calculation of coupled chemical equilibra. - Geoderma 38: 31-40.

RADDUM G. \& A. FJELLHEIM (1984): Acidification and early warning organisms in freshwater in western Norway. - Verh. Intern. Verein. Limnol. 22: 1973-1980.

RADDUM, G. \& A. FJELLHEIM (1987): Effects of $\mathrm{pH}$ and aluminium on mortality, drift and moulting of mayfly Baetis rhodani. - Annls. Soc. roy. zool. Belg. 117: 77-87.

RALEIGH, R.F. \& C. SHORT (1981): Depletion sampling in stream ecosystems: assumptions and techniques. - Prog. Fish-Cult. 43: 115-120.

RAMACHERS, B. \& D. BLANKE (1987): Beitrag zur Evertebratenfauna eines Waldbachs im niedersächsischen Bergland bei Hannover. - Beitr. Naturkd. Nds. 40/4: 237-248.

RAMAMOORTHY, S. (1988): Effect of $\mathrm{pH}$ on speciation and toxicity of aluminium to rainbow trout (Salmo gairdneri). - Can. J. Fish. Aquat. Sci. 45: 634-642.

RANDALL, D.J., S.F. PERRY \& T.A. HEMING (1982): Gas transfer and acid/base regulation in salmonids. - Comp. Biochem. Physiol. 73B: 93-103.

RATMEYER, V. (1990): Kartierung und limnogeologische Untersuchung von Sedimenten aus Sösestausee und Sösevorbecken (Sösemulde, Harz). Diplomarbeit Univ. Göttingen.

REHFELDT, G. (1987): Wirkung von Talsperren und Gewässerbelastung auf Invertebratengesellschaften in Fließgewässern und Auen des Harzes. Arch. Hydrobiol. 111: 255-281.

REICHENBACH-KLINKE, H.-H. (1956): Die Fischfauna des Oberharzes. - Natur und Jagd in Niedersachen, Sonderausgabe der Beiträge zur Naturkunde Niedersachsens.

REICHENBACH-KLINKE, H.-H. (1958): Weitere Mitteilung über die Fischfauna des Harzes. - Beitr. Naturkde. Nds. 11: 7.

REICHENBACH-KLINKE, H.-H. (1978): Zur Ökologie der Schwermetallanreicherung in Fischen. - Fisch und Umwelt 6: 7-11.

ROMMELMANN, J. \& U. HEITKAMP (1989): Auswirkungen der Gewässerversauerung auf die Chironomidenbesiedlung (Diptera: Chironomidae) in Quellbächen der Senne, Ostwestfalen. - Göttinger Naturk. Schr. 1: 131. 143. 
ROOSTAI, A.H. (1987): Geogene und anthropogene Quellen von Schwermetallen im Einzugsgebiet der Sösetalsperre (Westharz). - Diplomarbeit Univ. Hannover.

RÜDDENKLAU, R. (1989): Ökologische Untersuchungen an Trichopteren ausgewählter Harzbäche unter besonderer Berücksichtigung der Gewässerversauerung. - Diplomarbeit Univ. Göttingen.

RÜDDENKLAU, R. (1990): $V$. Trichpterenfauna. - In: HEITKAMP, U. \& D. LESSMANN (Hg.), Fallstudie Harz: Auswirkungen der Gewässerversauerung auf Bergbach- und Seebiozönosen. - Berichte des Forschungszentrums Waldökosysteme, Göttingen, Reihe B, Bd. 19: 97-194.

RÜDDENKLAU, R. (1991a): Vergleich von Ergebnissen aus Emergenz-, Lichtund Handnetzfängen adulter Köcherfliegen sowie Benthosaufsammlungen verschiedener Fließgewässer im Westharz. - Lauterbornia 8: 21-40.

RÜDDENKLAU, R. (1991b): Die bisher vom Gebiet des Harzes bekannten Trichopteren sowie einige Anmerkungen zu ihrer Zoogeographie und zu ihrem Gefährdungsgrad. - Göttinger naturkdl. Schr. 2: 5-13.

RUTTNER, F. (1962): Grundriß der Limnologie. - Berlin.

SANDROCK, F. (1978): Vergleichende Emergenzmessungen an zwei Bächen des Schlitzerlandes (Breitenbach und Rohrwiesenbach) 1970-1971. - Arch. Hydrobiol./Suppl. 54: 328-408.

SCHAEFER, M. \& W. TISCHLER (1983): Ökologie. - Wörterbücher der Biologie. Stuttgart.

SCHELLENBERG, A. (1942): Krebstiere oder Crustacea, IV. Flohkrebse oder Amphipoda. - in: F. DAHL (Hg.), Tierwelt Deutschlands 40/IV. Jena.

SCHIMMER, H. (1987a): Untersuchung tierischer Indikatoren in ausgewähiten, versauerungssensitiven Fließgewässern des Sauer- und Siegerlandes. Diplomarbeit Univ. Göttingen.

SCHIMMER, H. (1987b): Gammarus-Bestimmung leicht gemacht. - Il. Zoolog. Inst. Univ. Göttingen (unveröffentl.).

SCHIMMER, H. \& G. FRIEDRICH (1990): Die Auswirkungen der Gewässerversauerung auf das Makrozoobenthos ausgewählter Mittelgebirgsbäche im Sauer- und Siegerland. - Lauterbornia 5: 49-66.

SCHINDLER (1975): Unsere Süßwasserfische. - Stuttgart.

SCHMITZ, W. (1957): Die Bergbach-Zoozönosen und ihre Abgrenzung, dargestellt am Beispiel der oberen Fulda. - Arch. Hydrobiol. 53: 465-498.

SCHNOOR, J.L. \& W. STUMM (1986): The role of chemical weathering in the neutralisation of acidic deposition. - Schweiz. Z. Hydrol. 48: 171-195.

SCHOEN, R. (1985): Zum Nachweis depositionsbedingter Versauerung in kalkarmen Fließgewässern der Bundesrepublik Deutschland. - In: NATIONALPARKVERW. BAYER. WALD (Hg.), Wald und Wasser, 5. Tagungsbericht: 631-644.

SCHOEN, R. (1988): Gewässerversauerung in der Bundesrepublik Deutschland unter besonderer Berücksichtigung der hsistorischen Entwicklung im süddeutschen Raum und methodischer Probleme bei der Erfassung der Versauerung. - Umweltbundesamt Forschungsbericht Nr. 10204342.

SCHOEN, R., R.F. WRIGHT \& M. KRIETER (1983): Regional survey of freshwater acidification in West Germany (FRG). - Acid Rain Research, Norw. Inst. Water Res. Rep. 5/1983, Oslo.

SCHOENEMUND, E. (1930): Eintagsfliegen oder Ephemeroptera. - In: F. DAHL (Hg.), Tierwelt Deutschlands 19. Jena.

SCHÖLL, F. (1987): Limnofauna des Nationalparks Bayerischer Wald unter besonderer Berücksichtigung der Gewässerversauerung. - Diss. Univ. Bonn.

SCHRIMPF, E. \& F. FOECKLER (1985): Gammarids in streams of northwestern Bavaria, West Germany: 1. Prediction of their general occurence by selected hydrochemical variables. - Arch. Hydrobiol. 103: 479-486.

SCHUBÖ, W. \& H.-M. UEHLINGER (1986): SPSS ${ }^{X}$ - Handbuch der Programmversion 2.2. - Stuttgart, New York. 
SCHWEIGER, G. (1957): Die toxikologische Einwirkung von Schwermetallsalzen auf Fische und Fischnährtiere. - Arch. Fischereiwiss. 8: 54-78.

SCHWERDTFEGER, F. (1975): Ökologie der Tiere. III. Synökologie. - Hamburg, Berlin.

SCHWOERBEL, J. (1986): Methoden der Hydrobiologie. - Stuttgart.

SCULLION, J. \& R.W. EDWARDS (1980): The effects of coal industry pollutants on the macroinvertebrate fauna of small rivers in the South Wales coalfield. - Freshw. Biol. 10: 141-162.

SEGNER, H., R. MARTHALER \& M. LINNENBACH (1988): Growth, aluminium uptake and mucous cell morphometrics of early life stages of brown trout, Salmo trutta, in low pH water. - Environm. Biol. Fish. 21: 153159.

SIEBERT, H. (1988): Hydrogeologische Untersuchungen im Einzugsgebiet des Sösestausees (Westharz). - Diplomarbeit Univ. Hannover.

SIEBERT, H. \& H. VIERHUFF (1989): Hydrogeologische Untersuchungen im Einzugsgebiet des Sösestausees (Westharz). - Bericht der Bundesanstalt für Geowissenschaften und Rohstoffe, Hannover (unveröff.).

SIEWERS, U. \& A.H. ROOSTAI (1990): Schwermetallbilanz aus Immission und geogenem Anteil im Einzugsgebiet der Sösetalsperre/Harz. - Ber. Forschungszentrums. Waldökosysteme, Göttingen, Reihe B, Bd. 19.

SIMPSON, K.W., R.W. BODE \& J.R. COLQUHOUN (1985): The macroinvertebrate fauna of an acid-stressed headwater stream in the Adirondack Mountains, New York. - Freshw. Biol. 15: 671-681.

SINGER, P. \& D. FLIEDNER (1970): Landeskunde Niedersachsen. - München.

SINGER, R. (1982): Effects of acid precipitation on benthos. - In: F.M. D'ITRI (Hg.), Acid precipitation - effects on ecological systems. Ann Arbor.

SOWA, R. (1970): Sur la taxonomie de Rhithrogena semicolorata (Curtis) et de quelques especes voisines d'Europe continentale (Ephemeroptera: Heptageniidael. - Rev. Suisse Zool. 77: 895-920.

SPÄH, H. \& W. BEISENHERZ (1986): Wiederbesiedlung von Forellenbächen, erfolgreiche Versuche mit Groppen. - Mitt. LÖLF NRW 3, 1986: 28-34.

STEFFAN, A.W. (1958): Die deutschen Arten der Gattungen Elmis, Esolus, Oulimnius, Riolus, Aptyktophallus (Coleoptera: Dryopidea). - Beitr. Entom. 8: 122-178.

STEFFAN, A.W. (1961): Vergleichend-mikromorphologische Genitaluntersuchungen zur Klärung des phylogenetischen Verwandtschaftsverhältnisses der mitteleuropäischen Dryopoidea (Coleoptera). - Zool. Jb. Syst. 88: 255-354.

STEINBERG, L. (1983): Artenhilfsprogramm Groppe. - LÖLF NRW, Naturschutz praktisch, Merkblätter zum Biotop- und Artenschutz 36.

STOKES, P.M. (1986): Ecological effects of acidification on primary producers in aquatic systems. - Water, Air, Soil Poll. 30: 421-438.

STONER, J.H., A.S. GEE \& K.R. WADE (1984): The effects of acidification on the ecology of streams in the Upper Tywi Catchment in West Wales. Environment. Poll. A 35: 125-158.

STUMM, W., J.J. MORGAN \& J.L. SCHNOOR (1985): Saurer Regen, eine Folge der Störung hydrogeochemischer Kreisläufe. - Naturwissenschaften 70: 216-223.

SURBER, E.W. (1937): Rainbow trout and bottom fauna production in one mile of a stream. - Trans. Amer. Fish. Soc. 66: 193-202.

SUTCLIFFE, D.W. \& T.R. CARRICK (1973): Studies on mountain streams in the English Lake District. I. PH, calcium and the distribution of invertebrates in the River Duddon. - Freshw. Biol. 3: 437-462.

THOMAS, A. (1968): Sur la taxonomie de quelques especes d'Ecdyonurus du Sud-Ouest de la France (Ephemeroptera). - Ann. Limnol. 4: 51-71.

THORUP, J., T.M. IVERSEN, N.O. ABSALONSEN, T. HOLM, J. JESSEN \& T. OLSEN (1987): Life cycles of four species of Baetis (Epehmeroptera) in three Danish streams. - Arch. Hydrobiol. 109: 49-65. 
TIPPING, E. \& C.A. BACKES (1987): Some aspects of the chemical speciation of aluminium in acid surface waters. - In: BARTH (Hg.), Reversibility of acidification, Oxford: 30-33.

TOWNSEND, C.R., A.G.HILDREW \& J.FRANCIS (1983): Community structure in some southern English streams: the influence of physicochemical factors. - Freshw. Biol. 13: 521-544.

UBA, Umweltbundesamt (1984): Gewässerversauerung in der Bundesrepublik Deutschland. - Materialien $1 / 84$, Berlin.

UBA, Umweltbundesamt (1987): Gewässerversauerung in der Bundesrepublik Deutschland. - Texte 22/87.

UEHLINGER, H.-M. (1988): SPSS/PC + - Benutzerhandbuch. - Stuttgart, New York.

ULRICH, B. (1985): Natürliche und anthropogene Komponenten der Bodenversauerung. - Mitt. dt. Bodenkdl. Ges. 43: 159-187.

ULRICH, B. (1989): Waldökosystemforschung, Konzepte und Wege. - DVWK Mitt. 17: 7-23.

ULRICH, B. \& G. BÜTTNER (1985): Waldsterben - Konsequenzen für die forstliche und landwirtschaftliche Ertragskraft. - Inform. z. Raumentwicklung Waldsterben und Raumordnung 10: 879-892.

ULRICH, B. \& H. MEYER (1987): Chemischer Zustand der Waldböden Deutschlands zwischen 1920 und 1960, Ursachen und Tendenzen seiner Veränderung. - Ber. Forschungszentrum Waldökosysteme, Göttingen, Reihe B, Bd. 5.

ULRICH, B. \& V. MALESSA (1989): Tiefengradienten der Bodenversauerung. Z. Pflanzenernähr. Bodenkd. 152: 81-84.

WADE, K.R., S.J. ORMEROD \& A.S. GEE (1989): Classification and ordination of macroinvertebrate assemblages to predict stream acidity in upland Wales. - Hydrobiologia 171: 59-78.

WAGENBRETH, O. \& W. STEINER (1989): Geologische Streifzüge. - Leipzig.

WAGNER, S. (1989): Letale Waldschäden im Gebiet des "Ackers" (Hochharz). Forst Holz 18: 494-498.

WASSMANN, R. (1987): Untersuchungen zur organismischen Drift in Fließgewässern Südniedersachsens. - Diss. Univ. Göttingen.

WEATHERLEY, N.S. \& S.J. ORMEROD (1987): The impact of acidification on macroinvertebrate assemblages in Welsh streams: towards an empirical model. - Environm. Poll. 46: 233-240.

WEATHERLEY, N.S., S.J. ORMEROD, S.P. THOMAS \& R.W. EDWARDS (1988): The response of macroinvertebrates to experimental episodes of low pH with different forms of aluminium during a natural spate. - Hydrobiol. 169: 225-232.

WEATHERLEY, N.S., G.P. RUTT \& S.J. ORMEROD (1989): Densities of benthic macroinvertebrates in upland Welsh streams of different acidity and landuse. - Arch. Hydrobiol. 115: 417-431.

WELTON, J.S., C.A. MILLS \& E.L. RENDLE (1983): Food and habitat partitioning in two small benthic fishes, Noemacheilus barbatulus (L.) and Cottus gobio L. - Arch. Hydrobiol. 97: 434-454.

WESTERMANN, F. (1990): Limnologische Untersuchungen der Makrobenthonfauna unversauerter und versauerter Bäche im Hils (Weser-LeineBergland, Südniedersachsen). - Diplomarbeit Univ. Göttingen.

WILLERS, J. (1988): Plankton- und Benthon-Zoozönosen einer versauerten (Oderteich) und einer versauerungsgefährdeten Talsperre (Sösestausee) im Harz. - Diplomarbeit Univ. Göttingen.

WILLOUGHBY, L.G. (1988): The ecology of Baetis muticus and Baetis rhodani (Insecta, Ephemeroptera), with special emphasis on acid water backgrounds. - Int. Rev. ges. Hydrobiol. 73: 259-273.

WILLUTZKI, H. (1962): Zur Waldgeschichte und Vermoorung sowie über Rekurrenzflächen im Oberharz. - Nova Acta Leopoldiana N.F. 25: 1-51. 
WINTERBOURN, M.J., A.G. HILDREW \& A. BOX (1985): Structure and grazing of stone surface organic layers in some acid streams of southern England. - Freshw. Biol. 15: 363-374.

WIPPERFÜRTH, J. (1989): Untersuchungen zur Passierbarkeit von Rohr- und Rahmendurchlässen in ausgewählten Harzbächen durch Vertreter rheobionter Tiergruppen (Ephemeroptera, Plecoptera, Trichoptera, Pisces). - Diplomarbeit Univ. Göttingen.

WULFHORST, J. (1991): Vergleichende Untersuchungen an Flohkrebsen, Wasserkäfern, Eintagsfliegen- und Köcherfliegenlarven aus Moospolstern und dem hyporheischen Interstitial entlang eines Versauerungsgradienten in zwei Bächen des Westharzes. - In: DEUTSCHE GESELLSCHAFT FÜR LIMNOLOGIE (Hg.), Erweiterte Zusammenfassungen der Jahrestagung 1990 in Essen: 376-380.

WUNDSCH, H.H. (1922): Beiträge zur Biologie von Gammarus pulex. - Arch. Hydrobiol. 13: 478-531.

WÜSTEMANN, O. (1989): Die Fischfauna des Harzes - ökologisch betrachtet. In: HARZMUSEUM WERNIGERODE (Hg.), Der Harz, eine Landschaft stellt sich vor, 21: 12-16.

ZAHN, H. (1990): Monitoringprogramm für versauerte Gewässer durch Luftschadstoffe in der Bundesrepublik Deutschland im Rahmen der ECE. - Umweltforschungsplan des Bundesministers $f$. Umwelt, Naturschutz $u$. Reaktorsicherheit, Wasserwirtschaft-Forschungsbericht 10204362.

ZIEMANN, H. (1975): Über den Einfluß der Wasserstoffionenkonzentration und des Hydrogenkarbonatgehaltes auf die Ausbildung von Bergbachbiozönosen. - Int. Rev. ges. Hydrobiol. 60: 523-555.

ZUCCHI, H. \& A. GOLL (1981): Untersuchungen zum Einfluß wasserbaulicher Maßnahmen auf Süßwasserfische an Abschnitten der oberen Hase (Kreis Osnabrück). - Natur u. Landschaft 56: 430.

ZURWERRA, A., M. METZLER \& I. TOMKA (1987): Biochemical systematics and evolution of the European Heptageniidae (Ephemeroptera). - Arch. Hydrobiol. 109: 481-510.

ZWICK, P. (1967): Beitrag zur Kenntnis der Plecopteren-Fauna des Harzes. Mitt. dt. entomol. Ges. 26: 59-61. 
